\title{
Conceptual Design Report Proton Power Upgrade Project
}
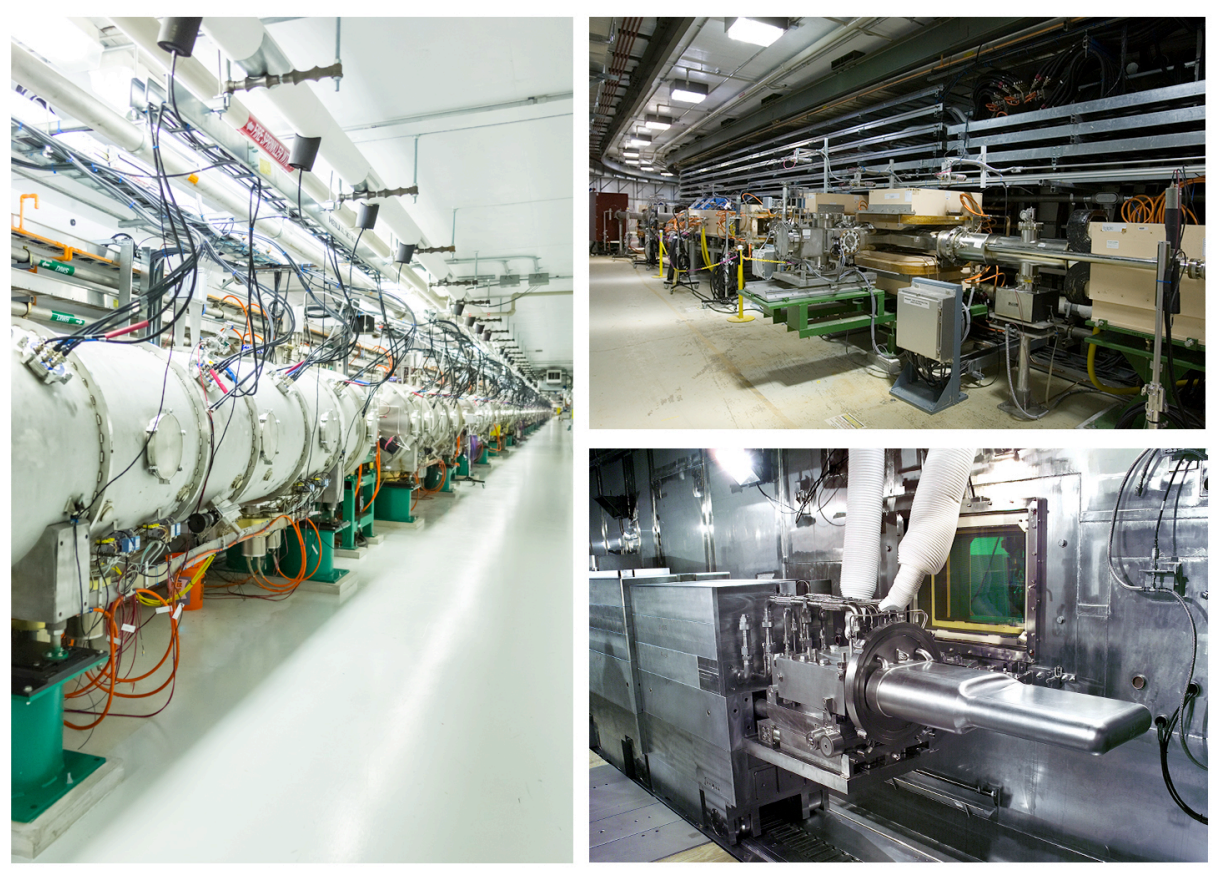

Approved for public release.

Distribution is unlimited.

Feb. 2018 


\title{
DOCUMENT AVAILABILITY
}

Reports produced after January 1, 1996, are generally available free via US Department of Energy (DOE) SciTech Connect.

Website http://www.osti.gov/scitech/

Reports produced before January 1, 1996, may be purchased by members of the public from the following source:

\author{
National Technical Information Service \\ 5285 Port Royal Road \\ Springfield, VA 22161 \\ Telephone 703-605-6000 (1-800-553-6847) \\ TDD 703-487-4639 \\ Fax 703-605-6900 \\ E-mail info@ntis.gov \\ Website http://classic.ntis.gov/
}

Reports are available to DOE employees, DOE contractors, Energy Technology Data Exchange representatives, and International Nuclear Information System representatives from the following source:

Office of Scientific and Technical Information

PO Box 62

Oak Ridge, TN 37831

Telephone 865-576-8401

Fax 865-576-5728

E-mail reports@osti.gov

Website http://www.osti.gov/contact.html

\begin{abstract}
This report was prepared as an account of work sponsored by an agency of the United States Government. Neither the United States Government nor any agency thereof, nor any of their employees, makes any warranty, express or implied, or assumes any legal liability or responsibility for the accuracy, completeness, or usefulness of any information, apparatus, product, or process disclosed, or represents that its use would not infringe privately owned rights. Reference herein to any specific commercial product, process, or service by trade name, trademark, manufacturer, or otherwise, does not necessarily constitute or imply its endorsement, recommendation, or favoring by the United States Government or any agency thereof. The views and opinions of authors expressed herein do not necessarily state or reflect those of the United States Government or any agency thereof.
\end{abstract}


Neutron Sciences Directorate

\title{
CONCEPTUAL DESIGN REPORT PROTON POWER UPGRADE PROJECT
}

John Galambos, David Anderson, Charlotte Barbier, Kursat Bekar, Douglas Bunch, H Bullman, Jr., Mark Buchanan, Gregory Capps, Mark Champion, Aaron Coleman, Richard Collins, Mark Connell, Sarah Cousineau, Mark Crofford, Douglas Curry, Robert Dean, Brian Degraff, Marc Doleans, Bob Eason, James Eckroth, Nicholas Evans, Franz Gallmeier, Mandy Greenwood, Melissa Harvey, Kenneth Herwig, Jeffrey Holmes, Matthew Howell, Ahmad Ibrahim, Lorelei Jacobs, Larry Jones, Yoon Kang, Wylie Keener, Sang-Ho Kim, Brian Kolar, William Kravitz, John Kristy, Paul Langan,

Geoffrey Laughon, Wei Lu, Kelly Mahoney, John Mammosser, Thomas McManamy, Mark Middendorf, John Moss, Greg Norman, Kevin Norris, Ed O’Neal, Chip Piller, Michael Plum, Klent Pope, Jeremy Price, Bernie Riemer, R Roseberry, Jr., Robert Saethre, James Schubert, Andrei Shishlo, C. Smith, Dennis Solley, Jared Spradling, Gregory Stephens, Christopher Stone, Barbara Thibadeau, Steven Trotter, Zhijun Wang, Mark Wezensky, Austin Wheat, Karen White, Derrick Williams, Drew Winder

Date Published: Feb. 2018

\author{
Prepared by \\ OAK RIDGE NATIONAL LABORATORY \\ Oak Ridge, TN 37831-6283 \\ managed by \\ UT-BATTELLE, LLC \\ for the \\ US DEPARTMENT OF ENERGY \\ under contract DE-AC05-00OR22725
}





\section{CONTENTS}

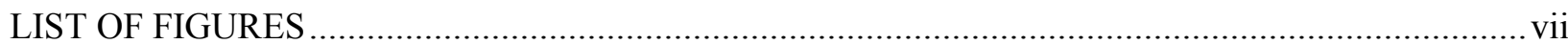

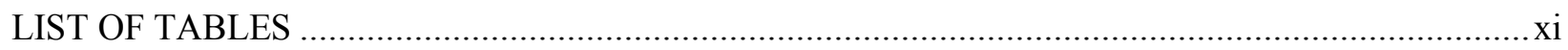

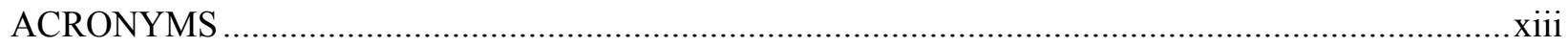

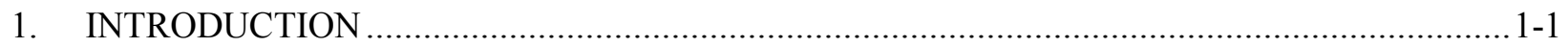

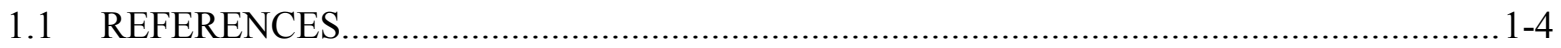

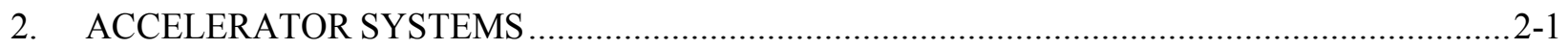

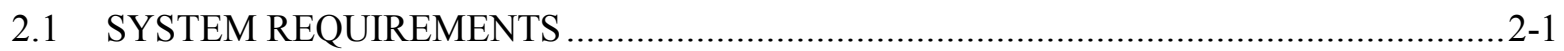

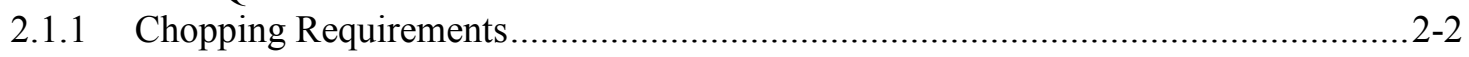

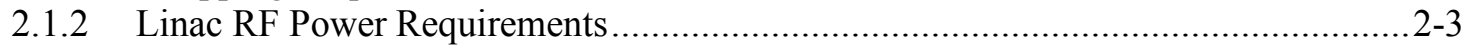

2.2 BEAM SIMULATIONS AND COLLECTIVE EFFECTS CONSIDERATIONS ................2-6

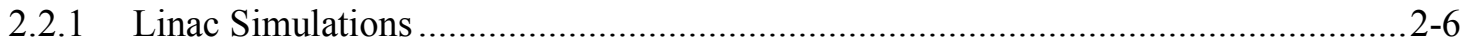

2.2.2 Ring and Ring-to-Target Beam Transport Simulations ......................................2-8

2.3 BEAM LOSS AND RESIDUAL ACTIVATION ................................................. 2-11

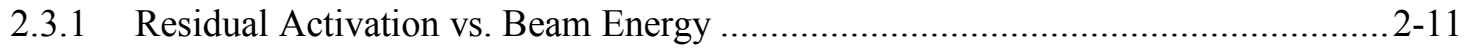

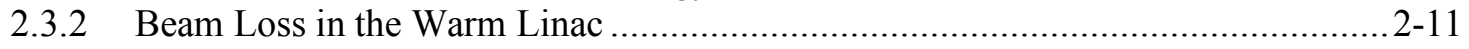

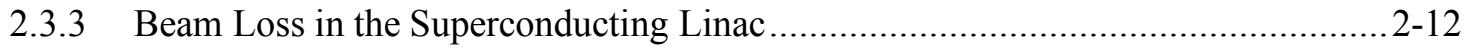

2.3.4 Beam Loss in the Ring Injection Area ............................................................ 2-12

2.3.5 Beam Loss in the HEBT and Ring-to-Target Beam Transport ............................. 2-14

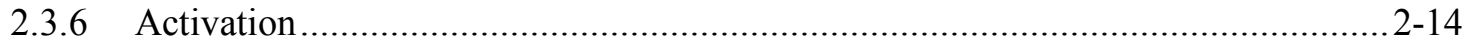

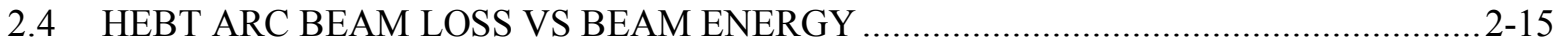

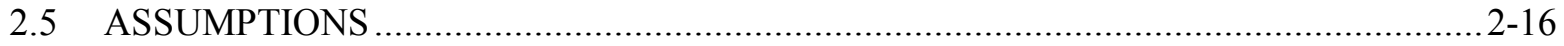

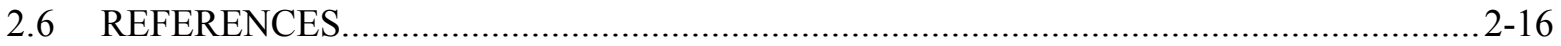

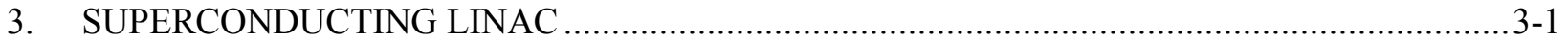

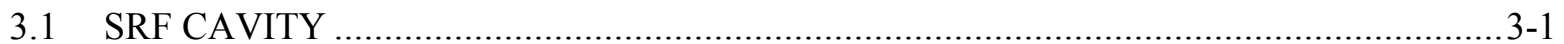

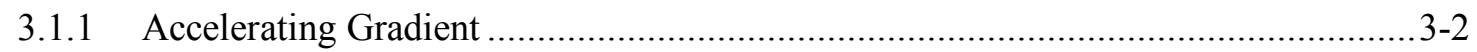

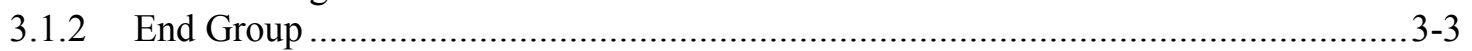

3.1.3 Fundamental Power Coupler .................................................................. 3-7

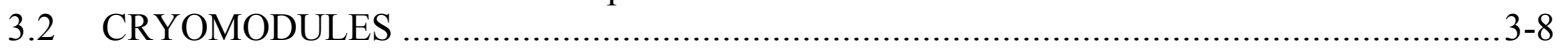

3.2.1 Head Loads and Helium Circuits............................................................... 3-11

3.2.2 Cold Mass................................................................................... $3-12$

3.2.3 Vacuum Vessel and Support Stands .......................................................... 3-14

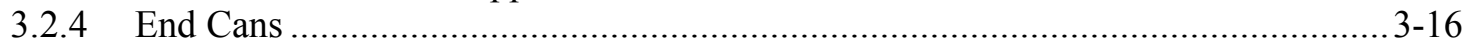

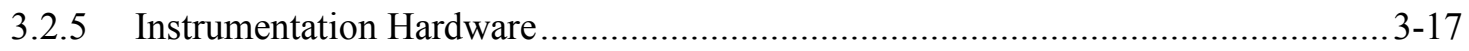

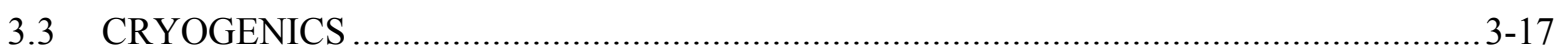

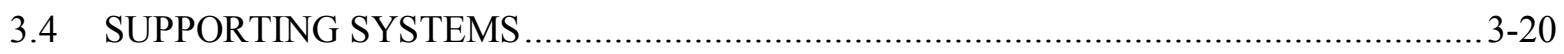

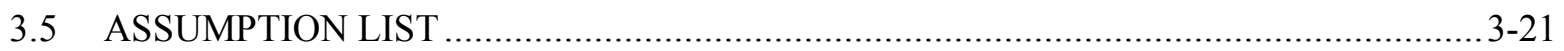

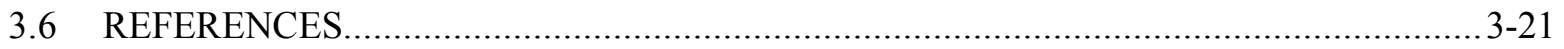

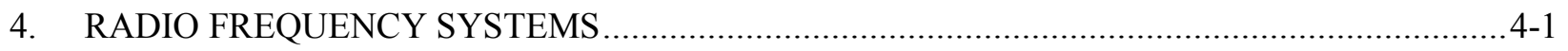

4.1 HIGH-POWER RADIO FREQUENCY SYSTEMS FOR THE PPU.............................. $4-1$

4.1.1 SCL High-Power Radio Frequency Systems..................................................... 4-1

4.1.2 Normal Conducting Linac High-Power Radio Frequency Systems......................... 4-6

4.2 LOW-LEVEL RADIO FREQUENCY SYSTEMS .................................................. $4-9$

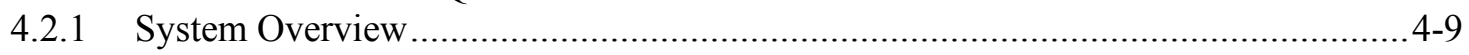

4.2.2 SCL LLRF Systems for PPU.................................................................... 4-11

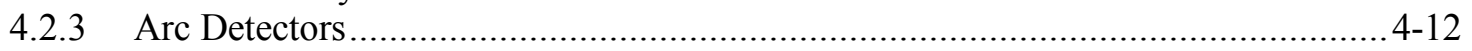

4.2.4 Reference Line................................................................................. $4-13$

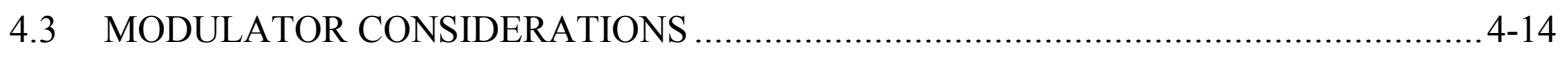

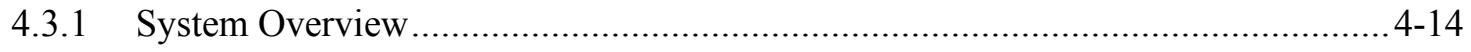


4.3.2 System Requirements and Interfaces ......................................................... 4-15

4.3.3 Existing Normal Conducting Linac Cavities....................................................... 4-17

4.3.4 New and Existing SCL Cavities .................................................................... 4-19

4.4 UTILITIES, NEW COLD LINAC RF COOLING SYSTEM .................................... 4-25

4.4.1 Upgrade of Existing SCL—RF Cooling Systems............................................ 4-27

4.4.2 Upgrade of Existing DTL RF Cooling System.................................................. 4-27

4.4.3 New Front End Building Cooling System...................................................... 4-27

4.4.4 Upgrade of Existing CCL RF Cooling System................................................... 4-28

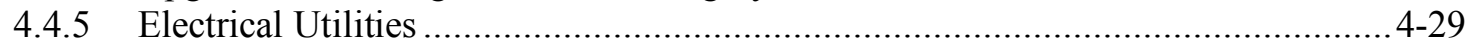

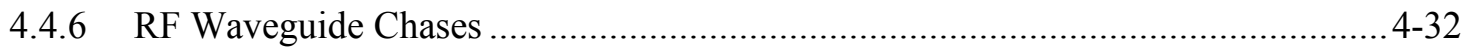

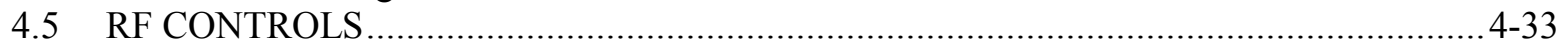

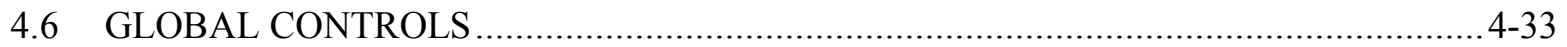

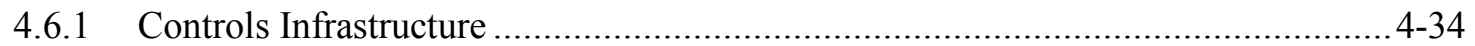

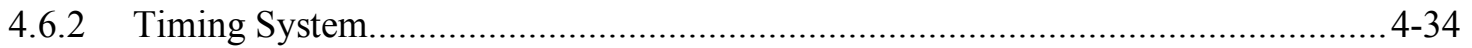

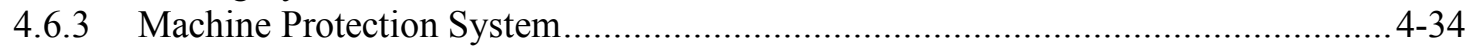

4.6.4 Personnel Protection Systems........................................................................... 4-34

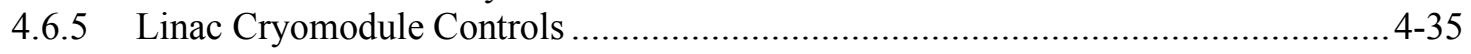

4.6.6 Linac Water System Controls..................................................................... 4-36

4.6.7 Beam Line Vacuum Control System ......................................................... 4-36

4.6.8 Insulating Vacuum System Controls....................................................................... 4-36

4.6.9 Ring Primary and Secondary Stripper Foil System Controls ............................... 4-36

4.6.10 Ring Water System Controls .................................................................... 4-36

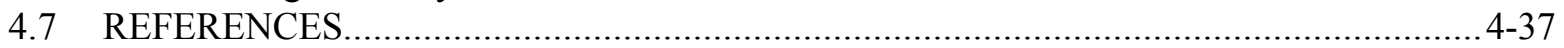

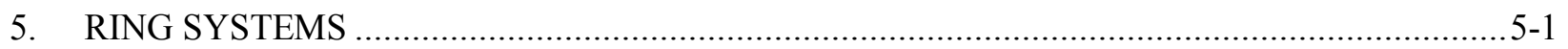

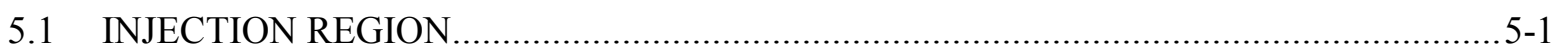

5.2 EXTRACTION REGION...........................................................................

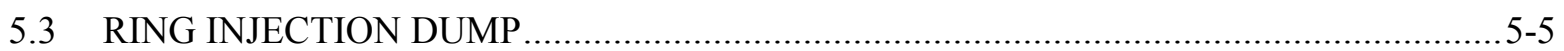

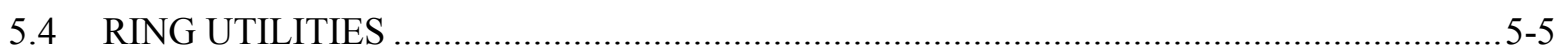

5.5 BEAM TRANSPORT LINES ……............................................................. $5-6$

5.5.1 High Energy Beam Transport Line ..................................................................... 5-6

5.5.2 Ring to Target Beam Transport Line ............................................................ 5-6

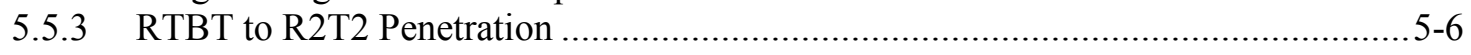

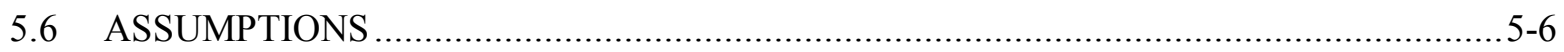

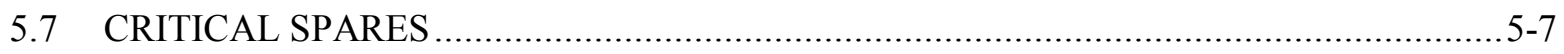

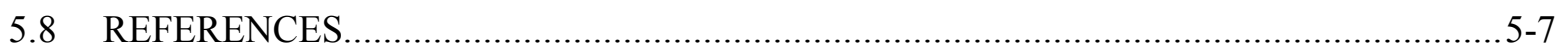

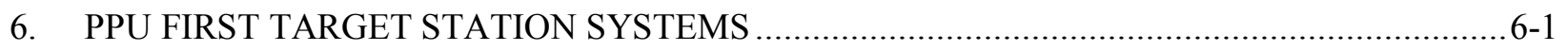

6.1 Overview of FTS Systems Evaluations and Upgrades ............................................... 6-1

6.1.1 FTS Systems Level 3 Summary Descriptions ............................................... 6-2

6.1.2 High-Flow Target Gas Injection .................................................................. $6-4$

6.2 DETAILED WORK SCOPE DESCRIPTIONS .................................................... $6-6$

6.2.1 Target Station Neutronics Evaluations and Upgrade Support ................................6-6

6.2.2 Mercury Process System Evaluation and Upgrades.......................................... 6-8

6.2.3 Moderator Cryogenic Systems Upgrades........................................................... 6-13

6.2.4 Evaluations of Reflector Vessel and Monolith Shielding Systems........................ 6-14

6.2.5 Target Utility Systems Evaluations and Upgrades ........................................... 6-16

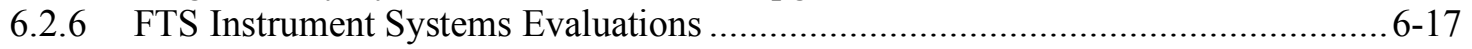

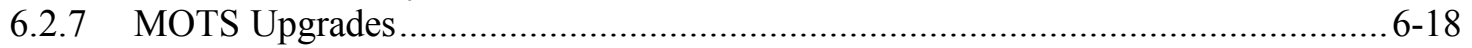

6.2.8 2.0 MW Target Module Design with High-Rate Gas Injection.............................. 6-19

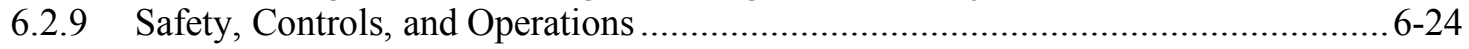

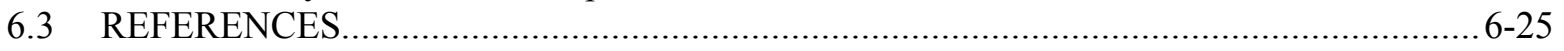




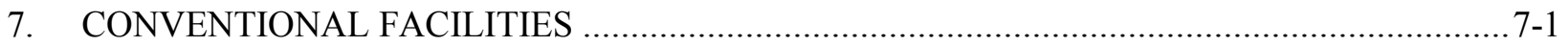

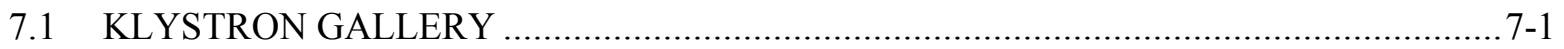

7.1.1 Klystron Gallery New Construction .......................................................... $7-2$

7.1.2 Klystron Gallery Existing Facility Modifications.................................................. $7-4$

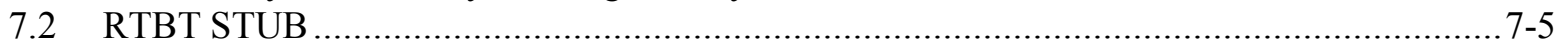

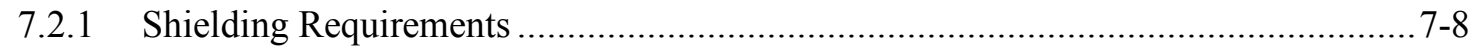

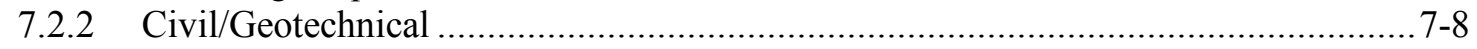

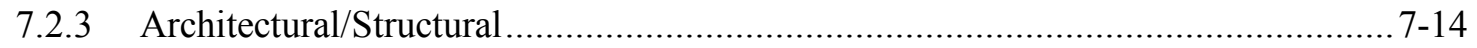

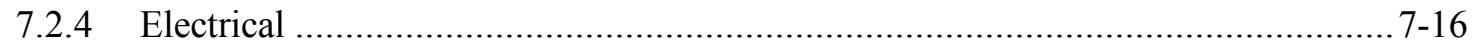

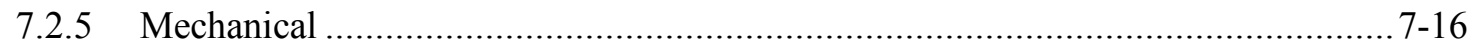

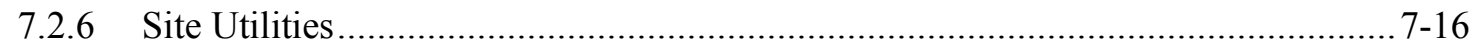

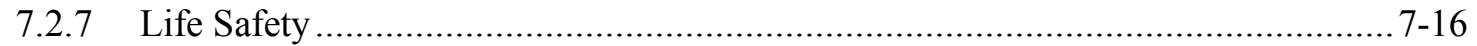

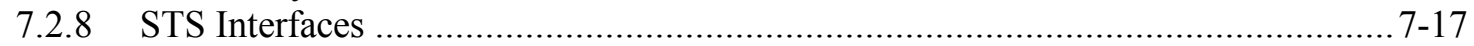

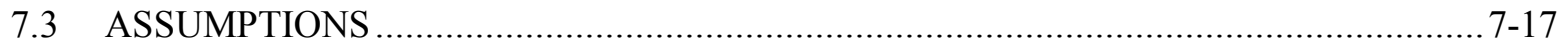

7.3.1 Klystron Gallery Assumptions ................................................................ $7-17$

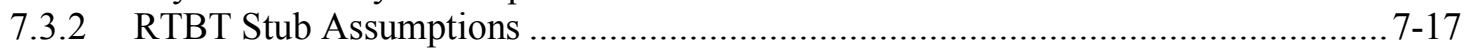

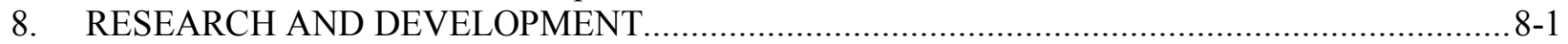

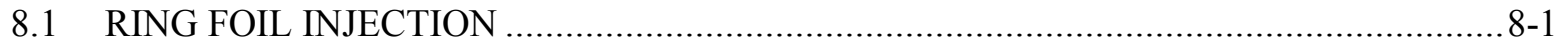

8.2 MERCURY TARGET HIGH-RATE GAS INJECTION DEVELOPMENT ...................... $8-1$

8.2.1 Small Gas Bubble Injection and Protective Gas Layer ........................................... 8-2

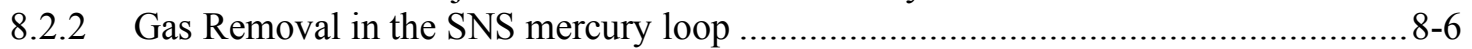

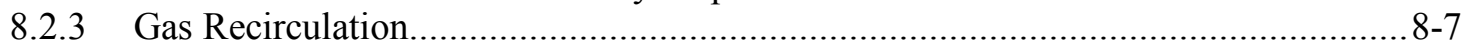

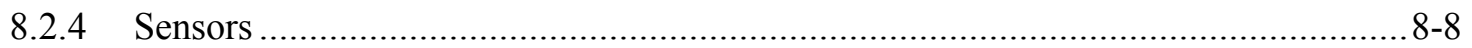

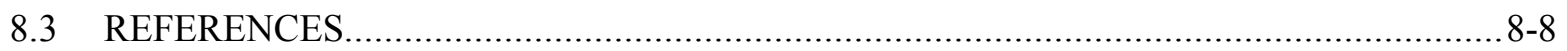

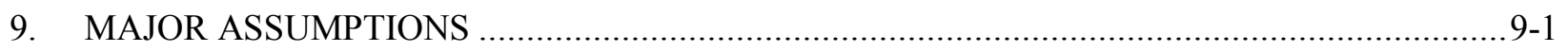

9.1 FRONT END SYSTEMS _.....................................................................

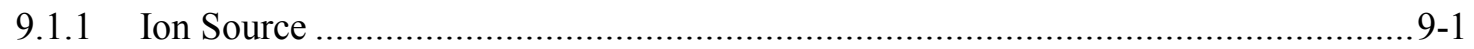

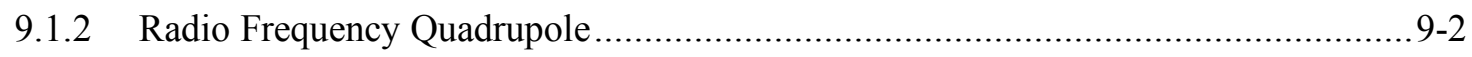

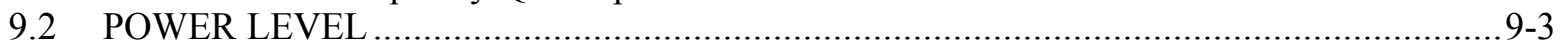

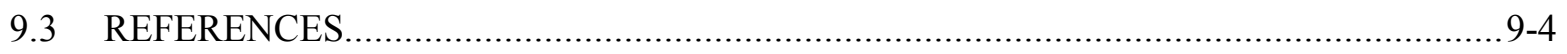

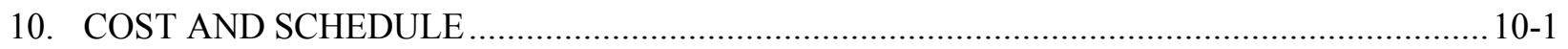

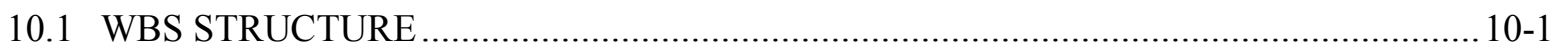

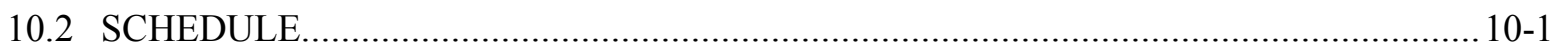

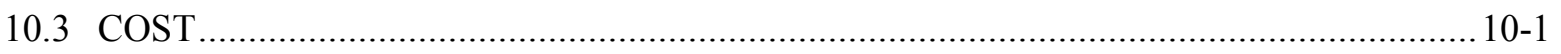





\section{LIST OF FIGURES}

Figure 2.1. Example pattern width variation over the ring injection cycle leading to a clean

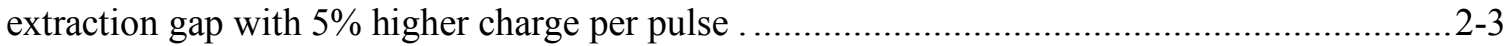

Figure 2.2. SCL cavity gradients after the PPU upgrade ............................................................2-4

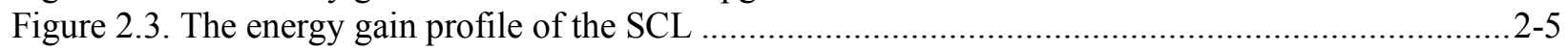

Figure 2.4. Required SCL klystron output power requirement vs. SCL cavity number .......................2-5

Figure 2.5. Transverse and longitudinal beam envelopes for the STS linac . .....................................2-6

Figure 2.6. Simulated rms emittance growth in the STS linac ..............................................2-7

Figure 2.7. Beam distribution at the HEBT entrance (blue) and at the primary stripper foil in the

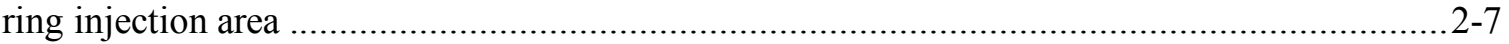

Figure 2.8. Beam distribution at the proton beam window upstream of the first target .....................2-8

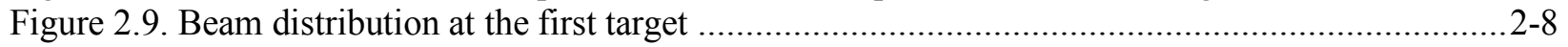

Figure 2.10. Activation as a function of beam energy .......................................................... 2-11

Figure 2.11. Recent dose rate data measured on September 22, 2015, after 1.3 MW operation ........... 2-14

Figure 2.12. Plot of the fractional loss per meter in the HEBT arc vs. beam energy . ..........................2-16

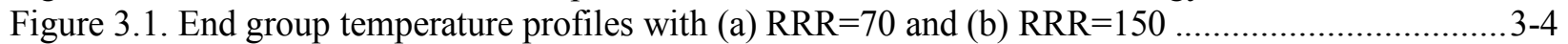

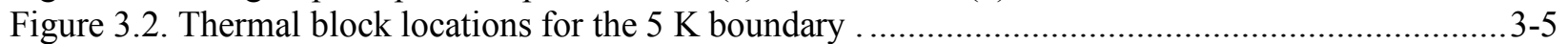

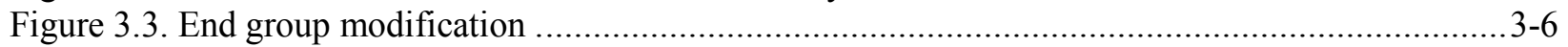

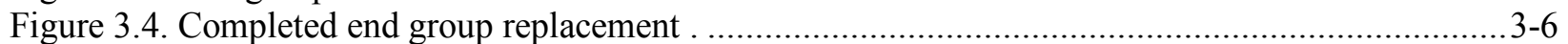

Figure 3.5. Original (left) versus new (right) helium vessel designs ............................................ 3-7

Figure 3.6. SNS fundamental power coupler (left: schematics, middle: inner conductor assembly,

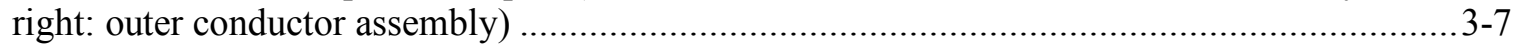

Figure 3.7. Comparisons of calculated inner conductor tip temperatures ......................................... 3-8

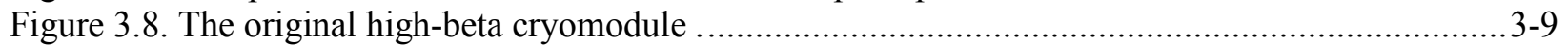

Figure 3.9. Cut view of the original high-beta cryomodule ..............................................................

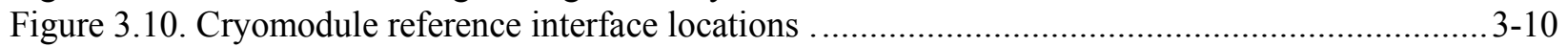

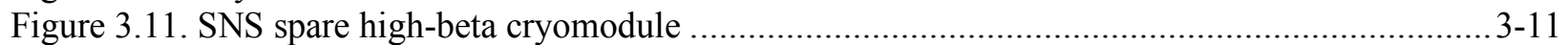

Figure 3.12. Helium flow schematic and instruments for the spare high-beta cryomodule ..................3-12

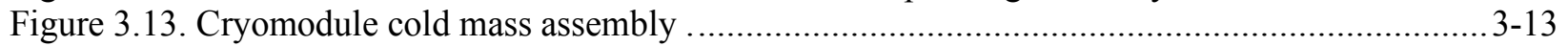

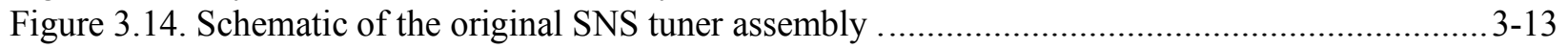

Figure 3.15. Original (left) versus new (right) vacuum vessel ................................................... 3-15

Figure 3.16. Original (left) versus new (right) vacuum jacket design ....................................... 3-15

Figure 3.17. JT valve positions for the PPU cryomodule (left) and the original (right) cryomodule...... 3-15

Figure 3.18. Original (left) versus new (right) supply end can ......................................................

Figure 3.19. Original (left) versus new (right) return end can ................................................. 3-16

Figure 3.20. Interfaces between end cans and vacuum vessel for the PPU cryomodule ...................... 3-17

Figure 3.21. Cryogenic system overview...................................................................... 3-18

Figure 4.1. The physical layout of PPU RF transmitter racks will be similar to that of the existing

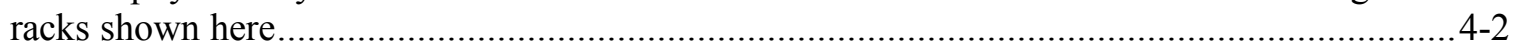

Figure 4.2. Estimated required RF generator power for the SCL .............................................. 4-3

Figure 4.3. Typical waveguide components. MEGA Industries 2016 [4] .......................................4-4

Figure 4.4. High-power waveguide circulator installed in an SCL transmitter at SNS ....................... 4-4

Figure 4.5. Water load installed in an SCL transmitter at SNS ..................................................... $4-5$

Figure 4.6. Top and side view of the redesigned TRCC ........................................................ $4-6$

Figure 4.7. Block diagram of the SNS low-level control system .................................................. $4-10$

Figure 4.8. The physical layout of the PPU LLRF racks will be similar to that of the existing

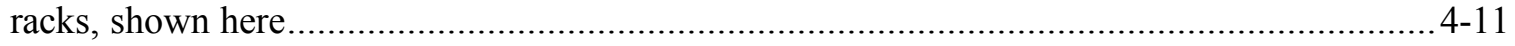

Figure 4.9. Advanced Ferrite Technology arc detector system............................................... 4-13

Figure 4.10. Master oscillator rack and block diagram............................................................. $4-13$ 
Figure 4.11. Block diagram of the HVCM system ............................................................... 4-14

Figure 4.12. Major components in the HVCM system ............................................................... 4-15

Figure 4.13. Operating voltage levels for existing SCL HVCMs to support PPU forward RF

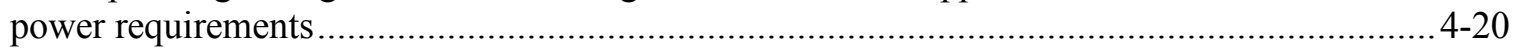

Figure 4.14. Block diagram of ATM topology (showing only a single phase) ................................. 4-21

Figure 4.15. PSpice simulation of the ATM IGBT operating waveforms ..................................... 4-21

Figure 4.16. ATM tank and other components under development ............................................ 4-23

Figure 4.17. Proposed KL-06 RF cooling system layout....................................................... 4-26

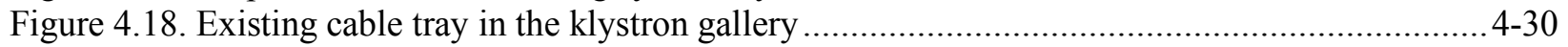

Figure 4.19. Existing ground-plane installation in the klystron gallery......................................... 4-31

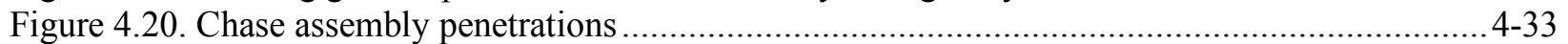

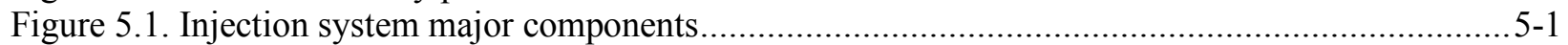

Figure 5.2. Stripping efficiency vs. foil thickness for a $1.3 \mathrm{GeV} \mathrm{H}^{-}$beam..................................... $5-2$

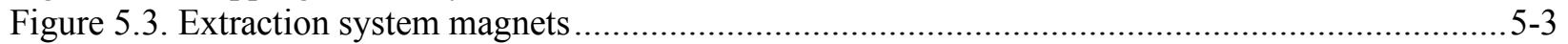

Figure 5.4. Schematic of the existing extraction kicker tank K1 with magnet types.............................5-3

Figure 5.5. Schematic of the present extraction kicker tank K2 with magnet types.............................. 5-3

Figure 5.6. Schematic of extraction kicker tank K1 with annex and renumbered magnets .....................5-4

Figure 5.7. Schematic of extraction kicker tank K2 with annex and renumbered magnets .................... 5-4

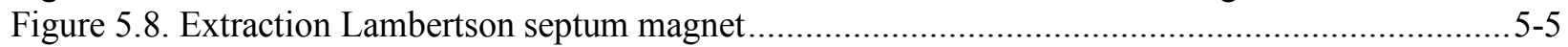

Figure 6.1. FTS Systems Work Breakdown Structure.................................................................... 6-2

Figure 6.2. Vibration data from Japan Spallation Neutron Source (JSNS) target illustrating the attenuating effects of gas bubble injection ........................................................................... $6-5$

Figure 6.3. Power deposition comparison in FTS for 1.3 and $1.0 \mathrm{GeV}$ at the target and IRP ................6-7

Figure 6.4. Target process bay (left) and mercury process loop (right)........................................... 6-9

Figure 6.5. Target carriage temperature profile at $2.0 \mathrm{MW}$ with a $1.0 \mathrm{GeV}$ beam............................. 6-10

Figure 6.6. Steady-state streamlines through the air flow around the target carriage........................... 6-10

Figure 6.7. Side and top views of the mercury process piping and heat exchanger showing regions that will likely accumulate gas as it leaves the target module ............................................... 6-11

Figure 6.8. The mercury pump model shows the upper side of the nozzle at 31.4 in. above the discharge nozzle elevation......................................................................................... $6-12$

Figure 6.9. Example hydro cyclone design type that might be adapted to mercury-gas separation

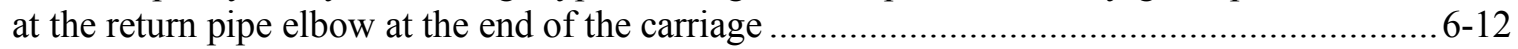

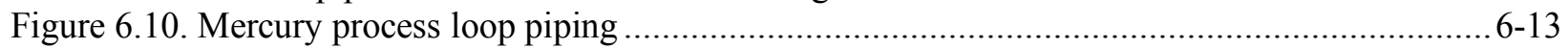

Figure 6.11. Monolith cross section showing vessel and monolith shielding systems ....................... 6-15

Figure 6.12. Temperatures in the ORP for the $1.0 \mathrm{GeV}, 2.0 \mathrm{MW}$ proton beam (left), and the temperatures in the core vessel target port for the $1.0 \mathrm{GeV}, 2.0 \mathrm{MW}$ proton beam.................. 6-16

Figure 6.13. Exploded view of target showing outer water cooled shroud and inner mercury

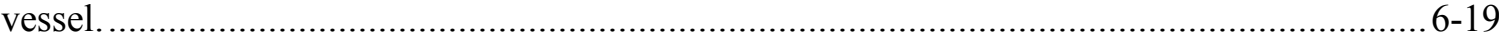

Figure 6.14. Current target mercury flow and structure ..................................................... 6-20

Figure 6.15. Cutaway showing target module in installed position.............................................. $6-21$

Figure 6.16. Cutaway view of nose of target mercury vessel showing the original mercury flow and the jet flow improvement that reduced cavitation damage ......................................... 6-22

Figure 6.17. Conceptual design of the forward and transition sections of a 2 MW PPU target............. 6-23

Figure 7.1. SNS campus map with detailed insert of the klystron gallery conventional facility

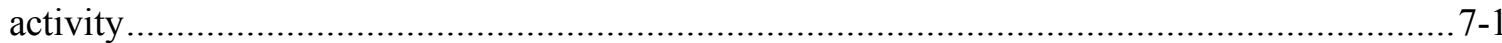

Figure 7.2. Aerial view of the Spallation Neutron Source campus …........................................... $7-6$

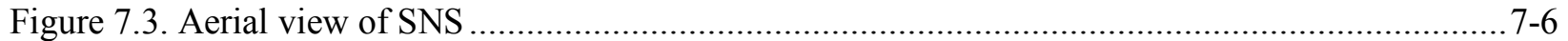

Figure 7.4. Existing RTBT tunnel looking toward the ring .......................................................... $7-7$

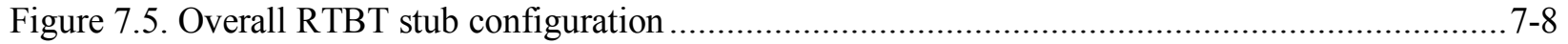

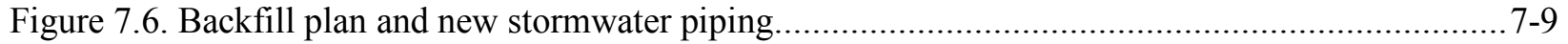

Figure 7.7. The existing RTBT tunnel with existing grades indicated ....................................... 7-10 


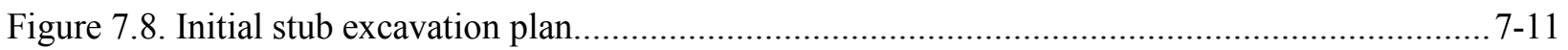

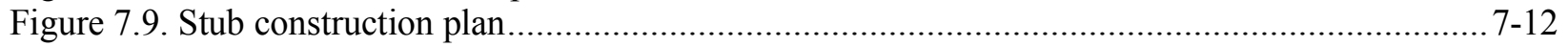

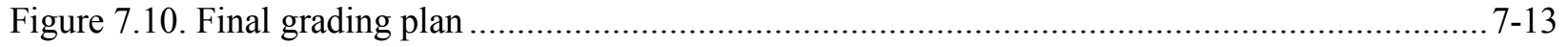

Figure 7.11. Elevation view of tunnel after final grading ............................................................ 7-14

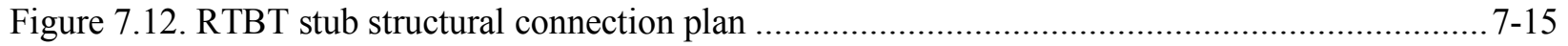

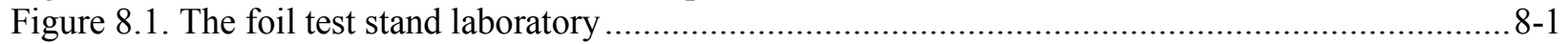

Figure 8.2. Photos of the mercury target at the TTF (left) and the water target at the THL .................. 8-2

Figure 8.3. Picture of the swirl bubblers assembly used at J-PARC (left) and schematic of the cross-section of a single swirl bubbler ............................................................................... 8-3

Figure 8.4. Swirl bubbler prototype developed for SNS target that was tested in a water loop (3D

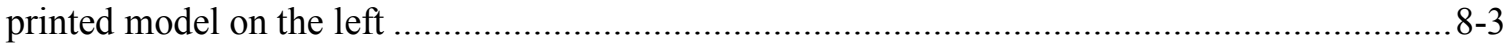

Figure 8.5. Free gas layer experiments at TTF on an original target design....................................... 8-4

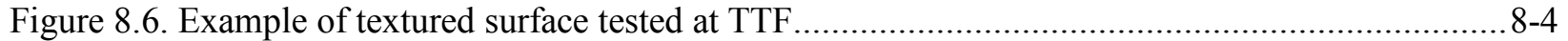

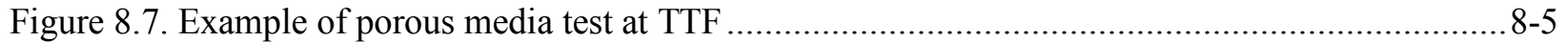

Figure 8.8. Schematic of the original and jet-flow target designs and their associated flow patterns ....... 8-5

Figure 8.9. Elevation views of the TTF mercury test loop (top) and SNS mercury process loop............8-6

Figure 8.10. Old (top) and new (bottom) location of the target vent line ........................................ 8-7

Figure 8.11. A double bellows compressor from Senior Aerospace (left) and a diaphragm compressor from KNF Process Pumps (right) ...................................................................... 8-8

Figure 9.1. Recent ion source current, measured at the RFQ entrance on the SNS front-end ................ 9-1

Figure 9.2. RFQ beam transmission from simulations for the design value field profile along the

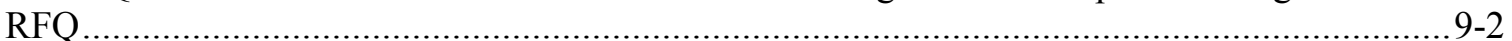

Figure 10.1. Level 3 Work Breakdown Structure for the Proton Power Upgrade................................. 10-2 



\section{LIST OF TABLES}

Table 1.1. High-level operational parameters for the PPU and current 1.4 MW operation.................... 1-3

Table 2.1. Present operational and upgraded parameters............................................................ 2-1

Table 2.2. Comparison of required front end parameters for the present PPU design and the

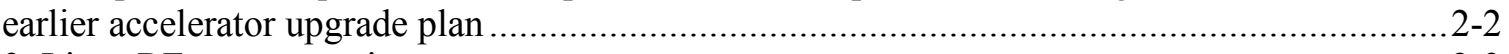

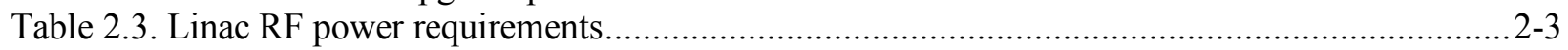

Table 2.4. Initial Twiss parameters for the linac simulation ...............................................................2-6

Table 2.5. Comparison of collective forces in SNS for four operating scenarios: present production, the original design, first target with an intensity upgrade, and second target

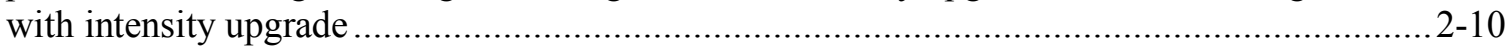

Table 2.6. Expected increase in dose rates from 1.4 to $2.8 \mathrm{MW}$ operation..................................... 2-15

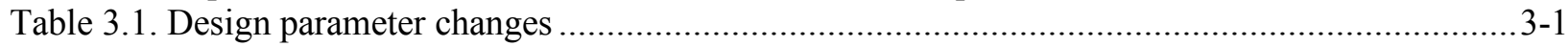

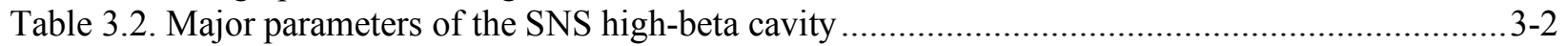

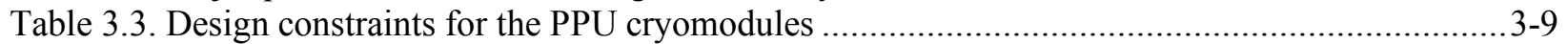

Table 3.4. Cryogenic load design values for the high-beta cryomodule............................................. 3-12

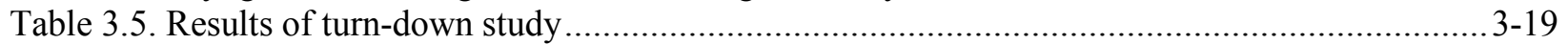

Table 4.1. Major PPU transmitter components ....................................................................................

Table 4.2. Measured structure parameters for the normal conducting linac ...................................... 4-7

Table 4.3. Estimated required RF generator power for the normal conducting linac ............................4-8

Table 4.4. Normal conducting linac high-power RF systems for the PPU ...................................... 4-9

Table 4.5. Commercially available components ......................................................................... 4-11

Table 4.6. Present and projected operating parameters for existing and new HVCM systems.............. 4-16

Table 4.7. HVCM requirements for new and existing systems..................................................... 4-17

Table 4.8. Comparison between current HVCM systems scaled to deliver power to the new PPU klystrons and an alternate topology modulator delivering the same output ........................... 4-22

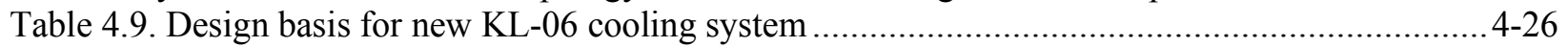

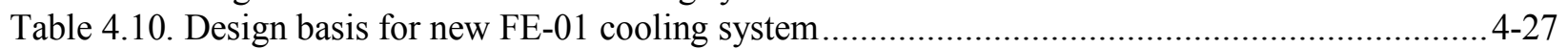

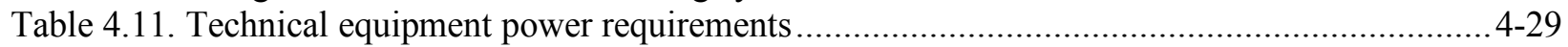

Table 4.12. Proposed additional power panels for PPU ............................................................... 4-30

Table 4.13. Preliminary description of cable needs for PPU electrical systems ................................ 4-31

Table 9.1. Beam parameters for Proton Power Upgrade options ...................................................... 9-4

Table 10.1. Proton Power Upgrade Critical Decision actual and planned milestones.......................... 10-1

Table 10.2. Proton Power Upgrade Project cost estimate ranges ................................................. 10-3

Table 10.3. Preliminary Budget Authority profile.......................................................................... 10-3 



\section{ABBREVIATIONS, ACRONYMS, AND INITIALISMS}

$\begin{array}{ll}\text { AC } & \text { alternating current } \\ \text { AFF } & \text { adaptive feed-forward } \\ \text { AIP } & \text { Accelerator Improvement Project } \\ \text { ASE } & \text { Accelerator Safety Envelope } \\ \text { ASME } & \text { American Society of Mechanical Engineers } \\ \text { ATM } & \text { alternate topology modulator } \\ \text { ATS } & \text { automatic transfer switch } \\ \text { B\&PV } & \text { Boiler and Pressure Vessel } \\ \text { BCP } & \text { buffered chemical processing } \\ \text { BLI } & \text { beam line interface } \\ \text { BTF } & \text { Beam Test Facility } \\ & \\ \text { CCL } & \text { coupled-cavity linac } \\ \text { CD } & \text { Critical Decision } \\ \text { CD-1 } & \text { Critical Decision 1 } \\ \text { CF } & \text { Conventional Facilities } \\ \text { CFD } & \text { computational fluid dynamics } \\ \text { CVI } & \text { core vessel insert } \\ \text { CW } & \text { continuous wave } \\ \text { DAC } & \text { design and analysis calculation } \\ \text { DC } & \text { direct current } \\ \text { DIWS } & \text { deionized water system } \\ \text { DOE } & \text { Department of Energy } \\ \text { DPA } & \text { displacements per atom } \\ \text { DTL } & \text { drift-tube linac } \\ & \\ \text { EIS } & \text { environmental impact statement } \\ \text { EL } & \text { event link } \\ \text { EP } & \text { electropolishing } \\ \text { EPICS } & \text { Experimental Physics and Industrial Control System } \\ \text { FCM } & \text { field control module } \\ \text { FEB } & \text { Front End Building } \\ \text { FPC } & \text { fundamental power coupler } \\ \text { FPGA } & \text { field-programmable gate array } \\ \text { FSAD } & \text { Final Safety Assessment Document } \\ \text { FTS } & \text { First Target System } \\ \text { GAR } & \text { gold amalgamation room } \\ \text { GI3 } & \text { gas injection initial implementation } \\ \text { GLS } & \text { gas-liquid separation } \\ \text { gpm } & \text { gallons per minute } \\ \text { HEBT } & \text { high-energy beam transport line } \\ \text { HEDP } & \text { high-energy differential pumping } \\ \text { HHRF } & \text { high-power radio frequency } \\ \text { HOM } & \text { higher-order mode } \\ \text { HP } & \text { high pressure } \\ \text { HPM } & \text { high-power protection module } \\ \text { HPRF } & \end{array}$




\begin{tabular}{|c|c|}
\hline $\begin{array}{l}\text { HUR } \\
\text { HVAC } \\
\text { HVCM }\end{array}$ & $\begin{array}{l}\text { hydrogen utility room } \\
\text { heating, ventilation, and air-conditioning } \\
\text { high voltage converter modulator }\end{array}$ \\
\hline $\begin{array}{l}\text { ICS } \\
\text { IEEE } \\
\text { IGBT } \\
\text { IOC } \\
\text { IRP } \\
\text { J-PARC } \\
\text { JT } \\
\text { LEBT } \\
\text { LED } \\
\text { linac } \\
\text { LL } \\
\text { LLRF } \\
\text { LO }\end{array}$ & $\begin{array}{l}\text { Integrated Control System } \\
\text { Institute of Electric and Electronics Engineers } \\
\text { insulated gate bipolar transistor } \\
\text { input-output controller } \\
\text { inner reflector plug } \\
\text { Japan Proton Accelerator Research Complex } \\
\text { Joule-Thomson } \\
\text { low-energy beam transport line } \\
\text { light-emitting diode } \\
\text { linear accelerator } \\
\text { live load } \\
\text { low-level radio frequency } \\
\text { local oscillator }\end{array}$ \\
\hline $\begin{array}{l}\text { MAWP } \\
\text { MC } \\
\text { MCS } \\
\text { MEBT } \\
\text { MO } \\
\text { MOTS } \\
\text { MP } \\
\text { MPS } \\
\text { MTBF } \\
\text { MW } \\
\text { NFPA } \\
\text { ORNL } \\
\text { ORP }\end{array}$ & $\begin{array}{l}\text { maximum allowable working pressure } \\
\text { Monte Carlo } \\
\text { moderator cryogenic system } \\
\text { medium energy beam transport } \\
\text { master oscillator } \\
\text { mercury off-gas treatment system } \\
\text { medium pressure } \\
\text { Machine Protection System } \\
\text { mean time between failures } \\
\text { megawatt } \\
\text { National Fire Protection Association } \\
\text { Oak Ridge National Laboratory } \\
\text { outer reflector plug }\end{array}$ \\
\hline $\begin{array}{l}\text { PFN } \\
\text { PI } \\
\text { PLC } \\
\text { PMB } \\
\text { PPS } \\
\text { PPU } \\
\text { PSID } \\
\text { PSIG } \\
\text { PUP } \\
\text { PW }\end{array}$ & $\begin{array}{l}\text { pulse forming network } \\
\text { proportional-integral } \\
\text { programmable logic controller } \\
\text { Performance Measurement Baseline } \\
\text { Personnel Protection System or pulses per second } \\
\text { Proton Power Upgrade } \\
\text { pounds per square inch differential } \\
\text { pounds per square inch gauge } \\
\text { Power Upgrade Project } \\
\text { pattern width }\end{array}$ \\
\hline $\begin{array}{l}\text { R\&D } \\
\text { R2T2 } \\
\text { RF } \\
\text { RFQ } \\
\text { RFTF } \\
\text { RRR } \\
\text { RTBT } \\
\text { RTDL }\end{array}$ & $\begin{array}{l}\text { research and development } \\
\text { ring to target two } \\
\text { radio frequency } \\
\text { radio frequency quadrupole } \\
\text { Radio Frequency Test Facility } \\
\text { residual resistivity ratio } \\
\text { ring-to-target beam transport } \\
\text { real time data link }\end{array}$ \\
\hline
\end{tabular}




$\begin{array}{ll}\text { SC } & \text { superconducting } \\ \text { SCL } & \text { superconducting linac } \\ \text { SCR } & \text { silicon-controlled rectifiers } \\ \text { SLPM } & \text { standard liters per minute } \\ \text { SNS } & \text { Spallation Neutron Source } \\ \text { SRF } & \text { superconducting radio frequency } \\ \text { STS } & \text { Second Target Station } \\ \text { TC } & \text { technical component } \\ \text { TESLA } & \text { Tera-Electron-Volt Energy Superconducting Linear Accelerator } \\ \text { THL } & \text { Thermal Hydraulic Laboratory } \\ \text { TRCC } & \text { transmitter cooling cart } \\ \text { TRIP } & \text { Target Reliability Improvement Program } \\ \text { TTF } & \text { Target Test Facility } \\ & \\ \text { UPS } & \text { uninterruptible power supply } \\ \text { VESDA } & \text { very early smoke detection apparatus } \\ \text { VSWR } & \text { voltage standing wave ratio } \\ \text { WBS } & \text { Work Breakdown Structure } \\ \text { ZCS } & \text { zero current switching } \\ \text { ZVS } & \text { zero voltage switching }\end{array}$





\section{INTRODUCTION}

Oak Ridge National Laboratory (ORNL) operates and develops world-leading neutron scattering user facilities as centers of scientific excellence, attracting the best researchers from universities, industry, and national laboratories to advance scientific discovery and solve challenging technology problems that are best addressed using neutrons. As the first megawatt-class pulsed neutron source, the ORNL Spallation Neutron Source (SNS) generates the world's most intense, highest-peak-brightness neutron beams, providing US researchers with capabilities that are unique in the world. The use of SNS beam lines now generates about twice as many high-impact publications per neutron scattering instrument as the nexthighest peer neutron facility. The Proton Power Upgrade (PPU) project is critical to keep SNS at the international forefront and ensure continued leadership by maximizing the neutron flux available at the First Target Station (FTS) and providing the capability to drive a Second Target Station (STS). [1] The PPU will upgrade the SNS accelerator complex to double its current proton beam power capability-from 1.4 to $2.8 \mathrm{MW}$. The $2 \mathrm{MW}$ delivered to the FTS will improve performance across the entire existing and future instrument suite, and the future STS [2] would provide a wholly new capability in the form of a transformative new source optimized to produce the world's highest peak brightness of cold neutrons.

Experiments at FTS are flux-limited, and demands for beam time far exceed the availability. The PPU will immediately impact FTS by increasing the number of experiments that can be conducted on current and future FTS instruments. This increase will accelerate the pace of science discovery that relies on neutrons to provide unique information on the structure and dynamics of new materials. The increase in neutron peak brightness provided by PPU will add new scientific capabilities, especially for experiments that benefit from ongoing simultaneous advances in neutron scattering methods and technologies and in data analysis, theory and computation. Current developments in sample environments, spin manipulation and instrument technologies, combined with the higher neutron flux and peak brightness enabled by the PPU, will extend the reach and scientific impact of neutron scattering methods. Examples of how gains in flux translate directly into better experimental parameters include smaller samples sizes, smaller gauge volumes, more extremes of sample conditions, better time resolution, better energy resolution, faster scanning speeds, and access to slower dynamics regimes.

For example, in soft matter research, PPU will make possible new time-resolved in operando measurements of the formation of complicated molecular architectures that have great promise for technological applications requiring highly tunable morphologies. It will extend the reach of studies of the dynamics of polymers and liquids by reducing sample size requirements. It will also make possible completely new types of measurements of in-plane surface structures in thin films and adhesives by making routine use of grazing incidence methods possible.

In functional materials research, the PPU will broaden the application of neutron scattering to study fundamental parameters that drive performance improvement in alloys and bulk metallic glass by making possible higher temporal, temperature, and/or spatial resolution. It will provide the rapid turnaround needed for characterizing 3D printing of polymer, metal, or ceramic parts. It will make possible routine in situ characterization of critical engineering structures, such as structural components of suspension bridges, that at present are too time consuming. The increase in flux will allow researchers to quantitatively study the structural and dynamic evolution of electrode and electrolyte materials in real systems. With more access to beam time, the cycle time between material synthesis, characterization, and improvement will be reduced.

PPU will make currently marginal experiments in quantum materials feasible. Examples include measurements under high pressures and high magnetic fields, and inelastic neutron scattering using polarization analysis. Combining the higher neutron flux provided by the PPU with new diamond anvil cells developed at ORNL and pulsed high-field magnet technologies will enable the exploration of 
hitherto unattainable phase space for neutron diffraction and spectroscopy, making possible new scientific discoveries. Combining polarization analysis with PPU-enhanced inelastic neutron scattering will provide valuable insight into the exotic properties of numerous quantum materials, including multiferroics, superconductors, and topological states.

In biology, the increase in neutron flux provided by the PPU will reduce sample size requirements, making possible crystallographic experiments that are not feasible at present. When this advance is combined with the in situ control of the neutron cross section of hydrogen provided by dynamic nuclear polarization, the possibilities are game-changing in terms of increasing throughput and enhancing the ability to directly visualize hydrogen during drug binding and enzyme catalysis. The nondestructive nature of neutron scattering, and the capability to use deuteration to highlight specific components or molecules, position neutrons to play a key role in addressing challenges in understanding specific components of complexes or pathways within living systems. This "in-cell neutron scattering" approach opens new lines of research. However, these techniques are signal-to-noise limited, and the PPU will provide the flux increases required for further development.

PPU will also provide a necessary platform for STS, which will be the world's highest-peak-brightness cold neutron source and will enable new scientific capabilities complementary to those of the ORNL High Flux Isotope Reactor (HFIR) and the FTS, especially in the areas of soft matter and chemistry. In combination, the FTS, STS, and HFIR will provide the United States with unrivaled capabilities, and unrivaled flexibility, for the foreseeable future. The US research community urgently needs the improved instrument performance and new science capabilities enabled by a PPU-powered FTS.

Provisions for an accelerator power increase were made in the original SNS project, and these are being leveraged to provide a cost-effective means of doubling the beam power. Additionally, accelerator developments and operational experience over the last 10 years of operation provide a strong technical basis for much of the design. The plan for doubling the proton beam intensity capability per pulse is to increase the beam energy from 0.97 to $1.3 \mathrm{GeV}$ and increase the average linear accelerator (linac) beam current from 26 to $38 \mathrm{~mA}$.

Three sets of beam parameters are shown in Table 1.1. The first column shows present operational parameters corresponding to $1.4 \mathrm{MW}$ operation. The "full accelerator upgrade capability" set of parameters corresponds to the ultimate power capability if all pulses were run at the maximum intensity possible. The beam pulses sent to the STS will be at full intensity. However, the accelerator will also supply the FTS with beam, and the FTS target systems are designed for 2.0 MW. Therefore, the pulses sent to the FTS will be reduced in intensity. The reduction in beam intensity per pulse is accomplished by increasing the beam macro-pulse chopping fraction on the front end (i.e., reducing the average beam current, as discussed in Section 2). For eventual operation with both FTS and STS, the linac chopping fraction will be modulated pulse-to-pulse, depending on the target destination of each pulse.

Since the PPU is designed for the extreme case of powering all pulses at the full STS peak intensity, it can accommodate a change in the ratio of pulses delivered to the FTS or the STS. The beam parameters for $60 \mathrm{~Hz}$ operation at $2.0 \mathrm{MW}$ to the FTS are also shown in Table 1.1. The equipment needed to modulate the beam intensity pulse-to-pulse will be included in the STS project, with the exception of some lowlevel radio frequency (LLRF) scope described in Section 4. 
Table 1.1. High-level operational parameters for the PPU and current 1.4 MW operation

\begin{tabular}{lccc}
\hline & $\begin{array}{c}\text { Current SNS } \\
\mathbf{1 . 4} \mathbf{~ M W}\end{array}$ & $\begin{array}{c}\text { Full accelerator } \\
\text { upgrade capability }\end{array}$ & $\begin{array}{c}\text { FTS 60 Hz } \\
\text { operation }\end{array}$ \\
\hline Proton beam power capability $(\mathrm{MW})$ & 1.4 & 2.8 & 2.0 \\
Beam energy $(\mathrm{GeV})$ & 0.97 & 1.3 & 1.3 \\
Proton pulse length on target $(\mu \mathrm{s})$ & 0.75 & 0.75 & 0.75 \\
RFQ $^{*}$ output peak beam current $(\mathrm{mA})$ & 33 & 46 & 46 \\
Average linac chopping fraction $(\%)$ & 22 & 18 & 41 \\
Average macropulse beam current $(\mathrm{mA})$ & 25 & 38 & 27 \\
Pulse repetition rate $(\mathrm{Hz})$ & 60 & 60 & 60 \\
Macropulse length $(\mathrm{ms})$ & 1 & 1 & 1 \\
Ring extraction beam gap $(\mathrm{ns})$ & 250 & 200 & 450 \\
High-beta cryomodules & 12 & 19 & 19 \\
Proton per pulse capability & $1.55 \times 10^{14}$ & $2.24 \times 10^{14}$ & $1.60 \times 10^{14}$ \\
Energy per pulse $(\mathrm{kJ})$ & 23 & 47 & 33 \\
\hline
\end{tabular}

$* \mathrm{RFQ}=$ radio frequency quadrupole

The range of operational frequency considered for the STS is 10-20 Hz. Between 6 and 12 pulses per second from the $60 \mathrm{~Hz}$ stream produced by the accelerator will be redirected to the STS, depending on the final STS frequency choice. The remaining 40-50 pulses per second from the accelerator will be directed to the FTS. If the STS operates at $20 \mathrm{~Hz}$, the pulses going to the FTS will also be at full intensity and correspond to $1.87 \mathrm{MW}$. If the STS operates at $10 \mathrm{~Hz}$, the remaining pulses directed toward the FTS will be reduced in intensity to correspond to $2 \mathrm{MW}$, as indicated in Table 1 . The PPU will deliver an accelerator capable of accommodating whichever operational frequency the STS adopts.

More effective use of $\mathrm{H}^{-}$beam chopping reduces the required peak ion source current from earlier plans, obviating the need to develop a dual ion source front-end system, as envisioned in earlier accelerator upgrade plans [5]. This approach, the accelerator physics basis for the upgrade, and the RF system requirements are described in Section 2.

The beam energy increase is accommodated by the addition of seven new superconducting RF (SRF) cryomodules in existing space at the end of the linac tunnel. The new cryomodules will be copies of the spare cryomodule built at SNS and installed in 2012. The PPU will use the same gradients as this operational spare cryomodule has demonstrated. Some existing SRF cavity performance will be improved using the ongoing in situ plasma processing campaign. The SRF scope is described in Section 3. The new SRF cryomodules will be powered by new RF sources installed in the end of the klystron gallery, which has sufficient space for this equipment. This RF equipment includes 28 new klystrons, 3 new high-voltage convertor modulators (HVCMs), and associated support equipment. Additionally, some existing RF source equipment will need to be upgraded to accommodate the increased beam loading. The RF scope is described in Section 4. Together, the SRF and RF systems make up the majority of the PPU scope.

More than $95 \%$ of the installed ring and transport systems are presently capable of $1.3 \mathrm{GeV}$ operation. Also, operational developments over the last10 years in the injection area and ring damper area provide a solid base for increased intensity in the ring. Upgrades are limited to the ring injection and extraction areas, described in Section 5. The ring injection upgrade will require new chicane magnets, which will be modifications of the present design. The extraction area upgrade involves adding exact copies of existing equipment. 
In addition to upgrading accelerator components, the PPU includes some Conventional Facilities (CF) and target system scope. The target system effort (Section 6) covers upgrading the mercury target vessel to handle a $2 \mathrm{MW}$ beam, adding a high-volume helium injection system to mitigate cavitation damage issues, and evaluating other target system components (e.g., shielding, cooling) to validate $2 \mathrm{MW}$ operation at $1.3 \mathrm{GeV}$. The CF scope (Section 7) includes outfitting the klystron gallery effort for installation of the energy upgrade technical equipment, adding a water pump room, and providing a tunnel stub in the transport line from the ring to the target to facilitate the subsequent connection to a future transport line to the STS target.

The technology deployed for the PPU is largely a direct extension of existing operational equipment and thus does not require significant research and development (R\&D). The primary area in which development is supported is a large-volume fraction gas injection system for the mercury target damage mitigation effort. This work is described in Section 8.

The technical basis for not including front-end system upgrades in the PPU scope is described in Section 9 (Assumptions). The rationale for doubling the SNS accelerator capability, and not proceeding further with power increases, is also described in Section 9.

Finally, the initial cost estimate is presented in Section 10. This is a snapshot of the estimate as preparations for Critical Decision 1 (CD-1) are being prepared. The cost estimate will continue to evolve as the designs and technical basis mature.

\subsection{REFERENCES}

[1] Subcommittee on Basic Energy Sciences Facilities Prioritization, Report of the Basic Energy Sciences Advisory Committee Subcommittee on BES Facilities Prioritization, February 26-27, 2013, US Department of Energy, Washington, DC, February 2013. DOI: http://science.energy.gov/ /media/bes/besac/pdf/Reports/BESAC_Facilities_Prioritization_Report_2 013.pdf

[2] J. Galambos, D. E. Anderson, D. Bechtol, et al., Technical Design Report, Second Target Station, ORNL/TM-2015/24, January 2015. Available at http://www.osti.gov/scitech/biblio/1185891Accessed. September 2016.

[3] S. Henderson, et al., "Status of the SNS Beam Power Upgrade Project," pp. 345-347 in Proceedings of EPAC 2006, Edinburgh, Scotland, June 26-30, 2006. Available at https://accelconf.web.cern.ch/accelconf/e06/PAPERS/MOPCH129.PDF. Accessed September 2016. 


\section{ACCELERATOR SYSTEMS}

The Proton Power Upgrade (PPU) project will double the beam power by doubling the energy delivered per pulse. The SNS accelerator was designed with a power upgrade in mind. Additional empty space was provided in the linac tunnel and in the radio frequency (RF) support building to accommodate the energy increase portion of the power upgrade.

A key aspect of the proposed accelerator upgrade is leveraging lessons learned during the initial 10 years of operation. For example, fewer SRF cryomodules are needed for the energy increase than originally planned, based largely on the experience with the successful spare cryomodule presently in operation. Also, by incorporating intelligent $\mathrm{H}^{-}$beam chopping patterns, ion source requirements have been reduced, obviating the need for a dual source development.

Much of the proposed additional accelerator equipment is of the same type as is presently in use throughout the accelerator. Thus, much of the accelerator scope does not involve development effort. One development area concerns the HVCMs, which supply modulated high-voltage waveforms for the RF generating sources. The increased beam loading associated with the higher beam intensity per pulse creates higher loads for this equipment, and some development is needed. Also, in the storage ring, the increased intensity raises some issues regarding the charge exchange injection process and space charge, which are discussed later in this section.

\subsection{SYSTEM REQUIREMENTS}

The accelerator systems will be upgraded from the baseline capability of 1.4 to $2.8 \mathrm{MW}$. This will be accomplished by a combination of increased beam energy and increased beam current. The beam energy, presently $0.97 \mathrm{GeV}$, will be increased to $1.3 \mathrm{GeV}$ by plasma processing of the existing superconducting linac (SCL) cavities and the addition of seven cryomodules. The average macropulse beam current (including the chopping effect) will be increased from 25 to $38 \mathrm{~mA}$ by improvements to the chopping scheme, replacement of the RF-quadrupole (RFQ) to improve transmission, and modest ion source improvements. Some minor modifications to the ring are also required to accommodate the higher beam energy. The First Target Station (FTS) will be upgraded to accept 2.0 MW of beam power. The SNS initial baseline and PPU design parameters are summarized in Table 2.1.

Table 2.1. Present operational and upgraded parameters

\begin{tabular}{|c|c|c|c|}
\hline Parameter & 1.4 MW operation & Upgraded FTS & PPU capability \\
\hline Beam power on target (MW) & 1.4 & 2.0 & 2.8 \\
\hline Beam energy $(\mathrm{GeV})$ & 0.97 & 1.3 & 1.3 \\
\hline Average linac current $(\mathrm{mA})^{a}$ & 1.6 & 1.6 & 2.3 \\
\hline Average linac macropulse current $(\mathrm{mA})^{a}$ & 25 & 27 & 38 \\
\hline 402.5 MHz, 2.5 MW klystrons & 7 & 5 & 5 \\
\hline RFQ peak output linac current (mA) & 33 & 46 & 46 \\
\hline Ion source output current $(\mathrm{mA})$ & 50 & 54 & 54 \\
\hline Ring chopping fraction & 0.25 & 0.41 & 0.18 \\
\hline 402.5 MHz, 3 MW klystrons & 0 & 2 & 2 \\
\hline $805 \mathrm{MHz}, 5 \mathrm{MW}$ klystrons & 4 & 4 & 4 \\
\hline $805 \mathrm{MHz}, 550 \mathrm{~kW}$ klystrons & 81 & 81 & 81 \\
\hline
\end{tabular}


Table 2.1. Present operational and upgraded parameters (continued)

\begin{tabular}{lccc}
\hline \multicolumn{1}{c}{ Parameter } & $\mathbf{1 . 4}$ MW operation & Upgraded FTS & PPU capability \\
\hline $805 \mathrm{MHz} 700 \mathrm{~kW}$ klystrons & 0 & 28 & 28 \\
SRF transmitter racks & 14 & 19 & 19 \\
SRF high-voltage converter modulators & 7 & 10 & 10 \\
High-beta cryomodules & 12 & 19 & 19 \\
\hline
\end{tabular}

${ }^{a}$ Assumes $5 \%$ of linac beam power is sent to injection dump owing to stripping inefficiency.

$\mathrm{SRF}=$ superconducting radio frequency

\subsubsection{Chopping Requirements}

The RFQ output peak current requirement ( $46 \mathrm{~mA})$ is substantially lower than the peak current requirement for the original power upgrade planning [1] (59 mA). Some ion source parameters for the present design are compared with those for the original power upgrade proposal in Table 2.2. The reduction in required peak current is driven primarily by a decrease in the fraction of beam chopped to provide a gap for fast extraction in the ring. In the original design, $30 \%$ of the beam was chopped, whereas in the present design, only $18 \%$ of the beam is chopped. We have demonstrated during highpower operation (1.4 MW) that it is possible to chop only $\sim 21 \%$ of the beam and still maintain low extraction beam loss. Simulations show that it is possible to further reduce the chopping fraction by varying the minipulse width (also referred to as pattern width [PW]) throughout the injection cycle. Figure 2.1 shows one example of a $\mathrm{PW}$ variation that increases the beam charge per pulse by $5 \%$ beyond the constant $\mathrm{PW}=51$ case presently used in operation, yet achieves good extraction efficiency. [2] Further gains in beam charge should be possible by also varying the RF buncher voltage during the ring injection time. Pursuing these measures greatly decreases the required peak ion source current, especially because the RFQ transmission decreases sharply above 60-70 mA input. With the present requirements, the existing RFQ design appears to be a feasible approach, and the required ion source output is a modest increase over present operation. An upgraded chopper system in the existing low-energy beam transport line (LEBT) chopper system is being designed and installed to allow variations in the minipulse width beyond the initial ramp as discussed above.

Table 2.2. Comparison of required front end parameters for the present PPU design and the earlier accelerator upgrade plan

\begin{tabular}{lcc}
\hline \multicolumn{1}{c}{ Parameter } & Original upgrade design & Present PPU design \\
\hline Ion source output $(\mathrm{mA})$ & 74 & 54 \\
RFQ output current $^{a}(\mathrm{~mA})$ & 59 & 46 \\
Chopping fraction & 0.30 & 0.18 \\
Average linac macro-pulse current $(\mathrm{mA})$ & 41 & 38 \\
\hline
\end{tabular}

${ }^{a}$ Calculated for transmission of a 0.25 pi-mm-mrad emittance input beam with the design field profile along the RFQ. 


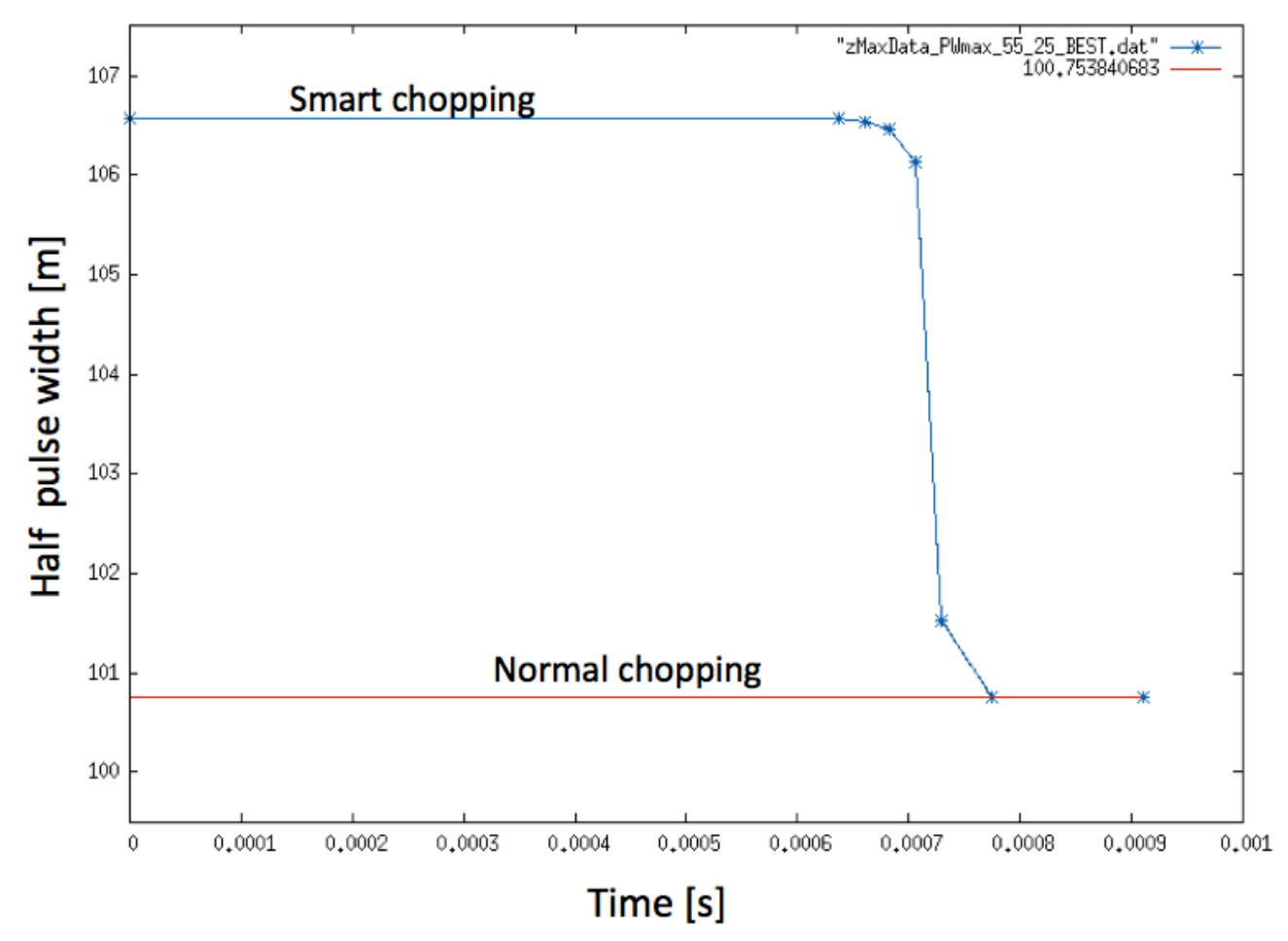

Figure 2.1. Example pattern width variation over the ring injection cycle leading to a clean extraction gap with $5 \%$ higher charge per pulse. Blue line: pattern width variation that results in higher beam charge. Red line: example of constant pattern width in use today.

\subsubsection{Linac RF Power Requirements}

The linac RF cavities will require more RF power to maintain the same accelerating gradient at the higher PPU beam currents. In the warm linac, it is not possible to mitigate the power increase by lowering the gradient. This is so because the geometry of the cavities dictates the beam energy for each cavity, and because many cells within the cavities are powered by a single klystron, which fixes the RF phase at each cell. Therefore, the only option is to increase the RF power delivered to each cavity. The existing RF power margin is not sufficient, so the RF systems will be upgraded to support an acceleration of the $38 \mathrm{~mA}$ average linac macropulse beam current. The linac RF power requirements are summarized in Table 2.3.

Table 2.3. Linac RF power requirements

\begin{tabular}{ll}
\hline \multicolumn{1}{c}{ System } & \multicolumn{1}{c}{ RF power requirement } \\
\hline Warm linac $402.5 \mathrm{MHz}$ & Upgrade to support $38 \mathrm{~mA}$ avg. macropulse beam current \\
Warm linac $805 \mathrm{MHz}$ & Upgrade to support $38 \mathrm{~mA}$ avg. macropulse beam current \\
SCL $805 \mathrm{MHz}$ - existing & Upgrade to support $38 \mathrm{~mA}$ avg. macropulse beam current at cavity \\
cryomodules & gradients shown in Figure 3.2. \\
SCL 805 MHz-new & New RF systems to support $38 \mathrm{~mA}$ avg. macropulse beam current at 16 \\
cryomodules & MV/m gradient \\
\hline
\end{tabular}

In the SCL there is more flexibility because there are only six cells per cavity (and one cavity per klystron). To support the acceleration of a $38 \mathrm{~mA}$ beam current (averaged over the macropulse), some SCL operational gradients will be decreased because the RF power systems are already close to their 
maximum output powers. Other SCL cavities that are not RF power-limited will have increased gradients. Plasma processing, which is now being developed, will allow these gradient increases (see Chapter 3). The seven new cryomodules will take advantage of klystron and coupler improvements that raise the maximum output power from 550 to $700 \mathrm{~kW}$. Figure 2.2 shows the SCL RF cavity gradients after the PPU upgrade. The resultant energy gain profile is shown in Figure 2.3. The latter shows an output beam energy of $1,343 \mathrm{MeV}$, but in practice the capability above $1,300 \mathrm{MeV}$ will be used as reserve capacity. The corresponding SCL klystron output power requirements are shown in Figure 2.4, including the beam loading and a control margin of $15 \%$.

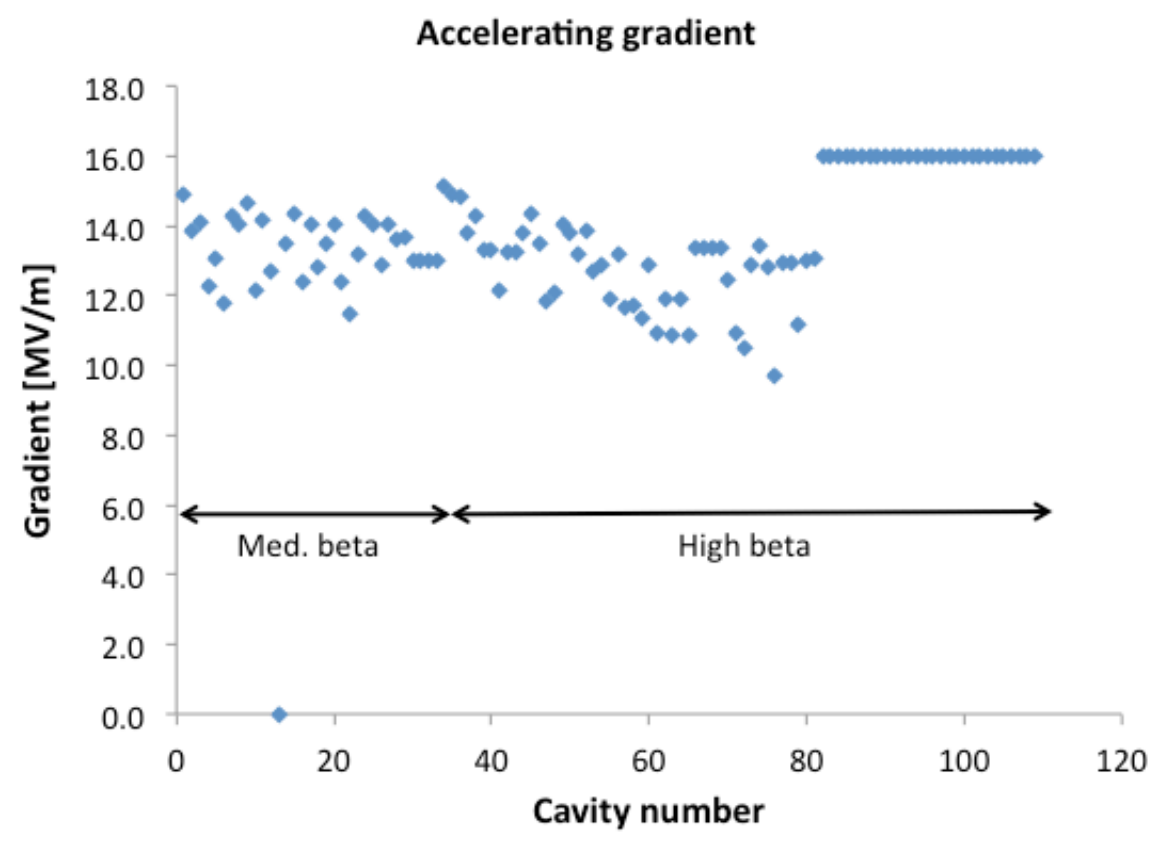

Figure 2.2. SCL cavity gradients after the PPU upgrade. 


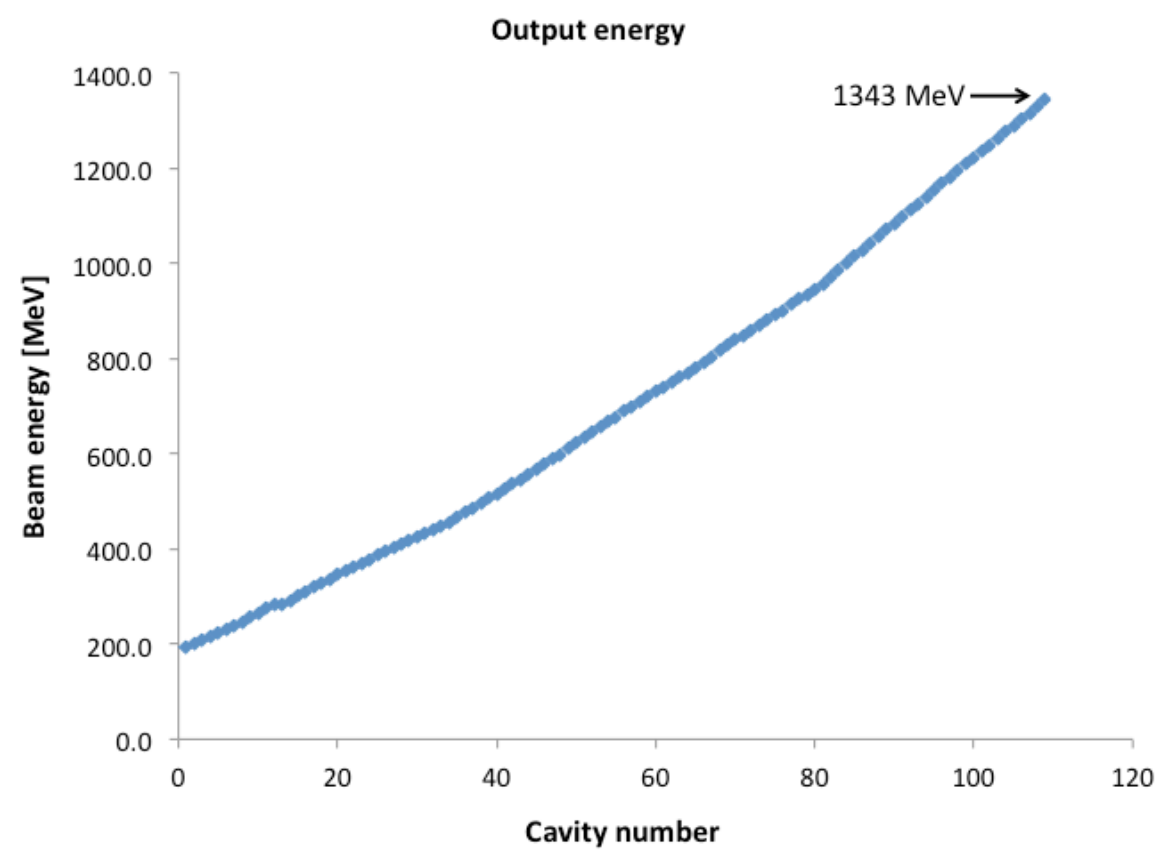

Figure 2.3. The energy gain profile of the SCL. The output energy of 1,343 $\mathrm{MeV}$ includes the reserve energy gain from the last three cavities.

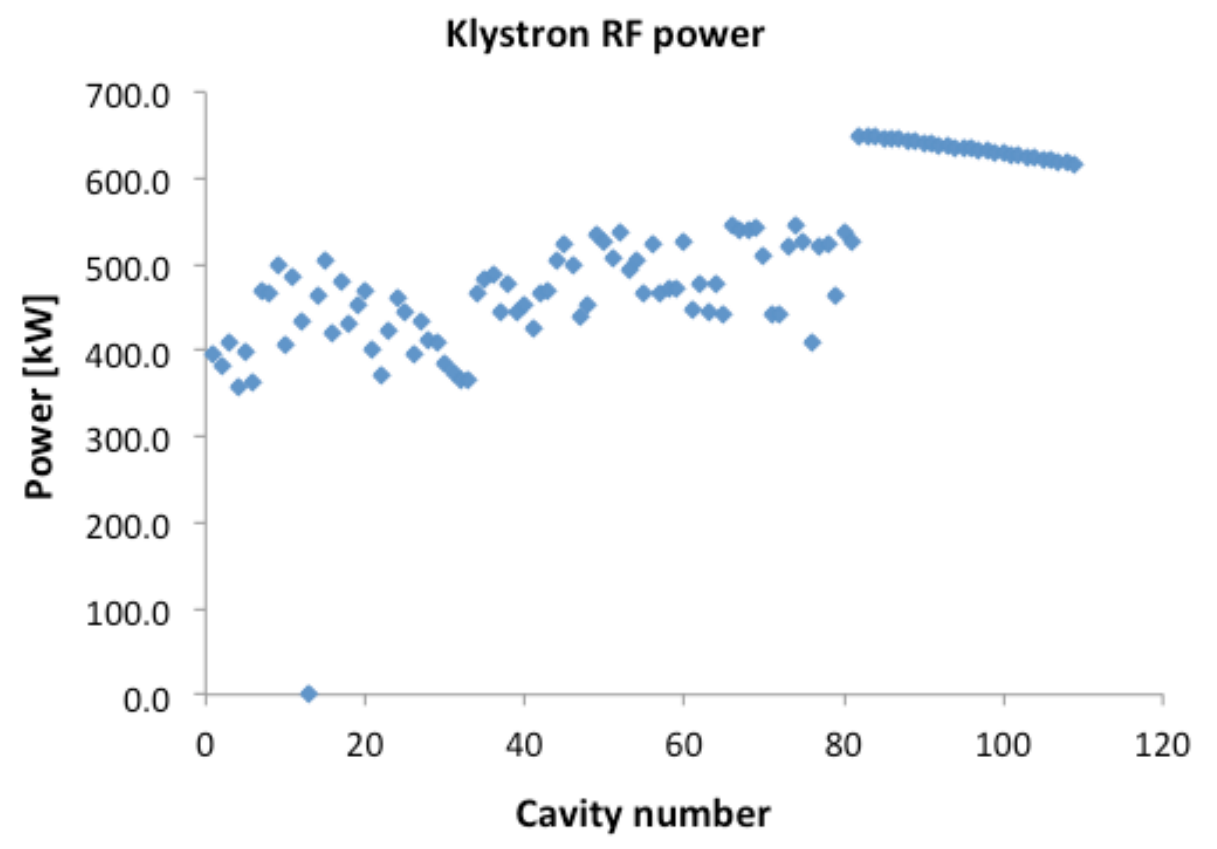

Figure 2.4. Required SCL klystron output power requirement vs. SCL cavity number. 


\subsection{BEAM SIMULATIONS AND COLLECTIVE EFFECTS CONSIDERATIONS}

\subsubsection{Linac Simulations}

Particle tracking simulations [3] have been performed for the PPU linac to verify the new linac design (personal communication from Z. Wang, Institute of Modern Physics, Lanzhou, China, to M. Plum, ORNL, May 2014). One million particles were tracked from the exit of the RFQ to the exit of the SCL. The initial particle distribution was assumed to be a 3-sigma Gaussian with Twiss parameters, taken from the standard SNS baseline case and reproduced in Table 2.4. The beam current was assumed to be $50 \mathrm{~mA}$ and the beam energy $2.5 \mathrm{MeV}$ (design output energy of the RFQ).

Table 2.4. Initial Twiss parameters for the linac simulation

\begin{tabular}{lccc}
\hline & Alpha & Beta $(\mathbf{m})$ & Emittance (pi-mm-mrad) \\
\hline $\mathrm{X}$ & -1.9619 & 0.18314 & 0.21 \\
$\mathrm{Y}$ & 1.7681 & 0.16203 & 0.21 \\
$\mathrm{Z}$ & 0.0196 & 0.58 & 0.24153 \\
\hline
\end{tabular}

The resultant transverse and longitudinal beam envelopes are shown in Figure 2.5, and the rms emittance growth is shown in Figure 2.6. The simulation results are close to expectations and do not present any problems.
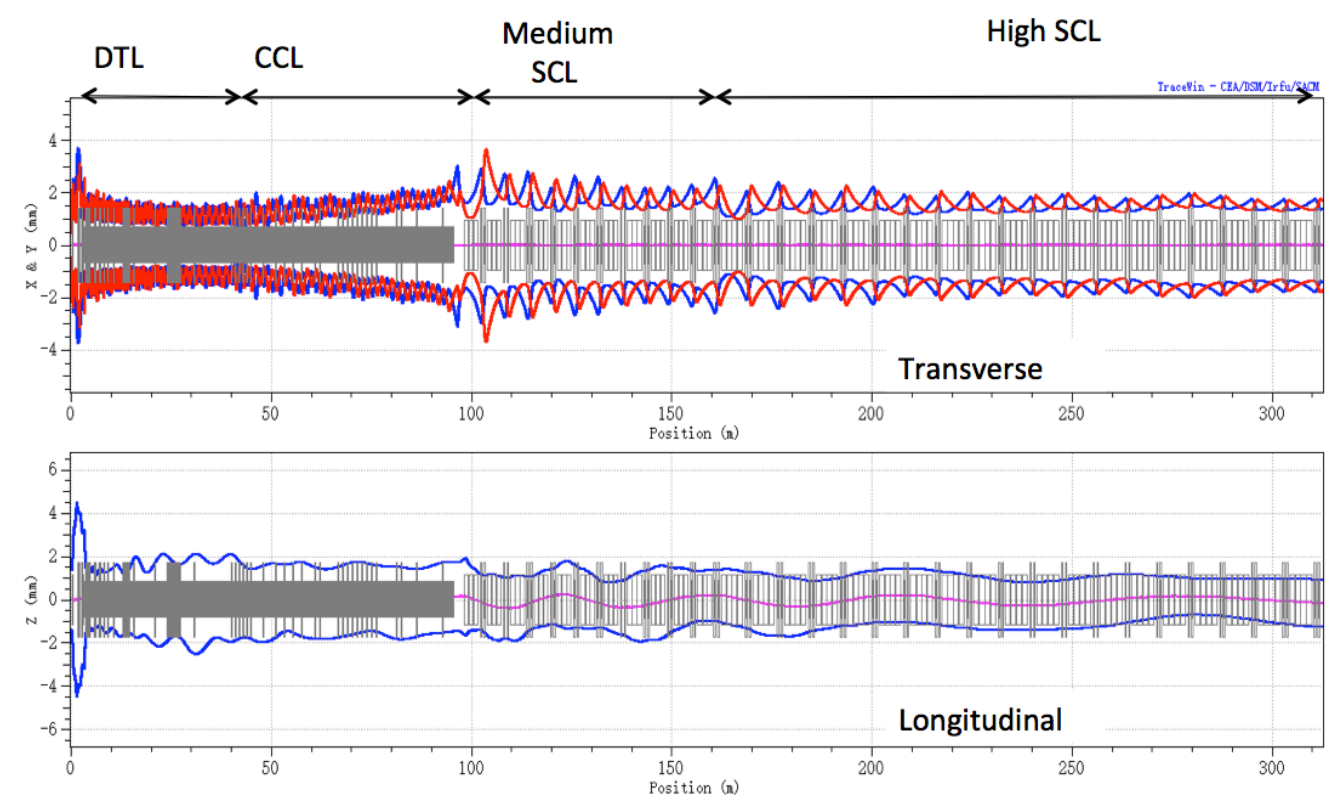

Figure 2.5. Transverse and longitudinal beam envelopes for the STS linac. 


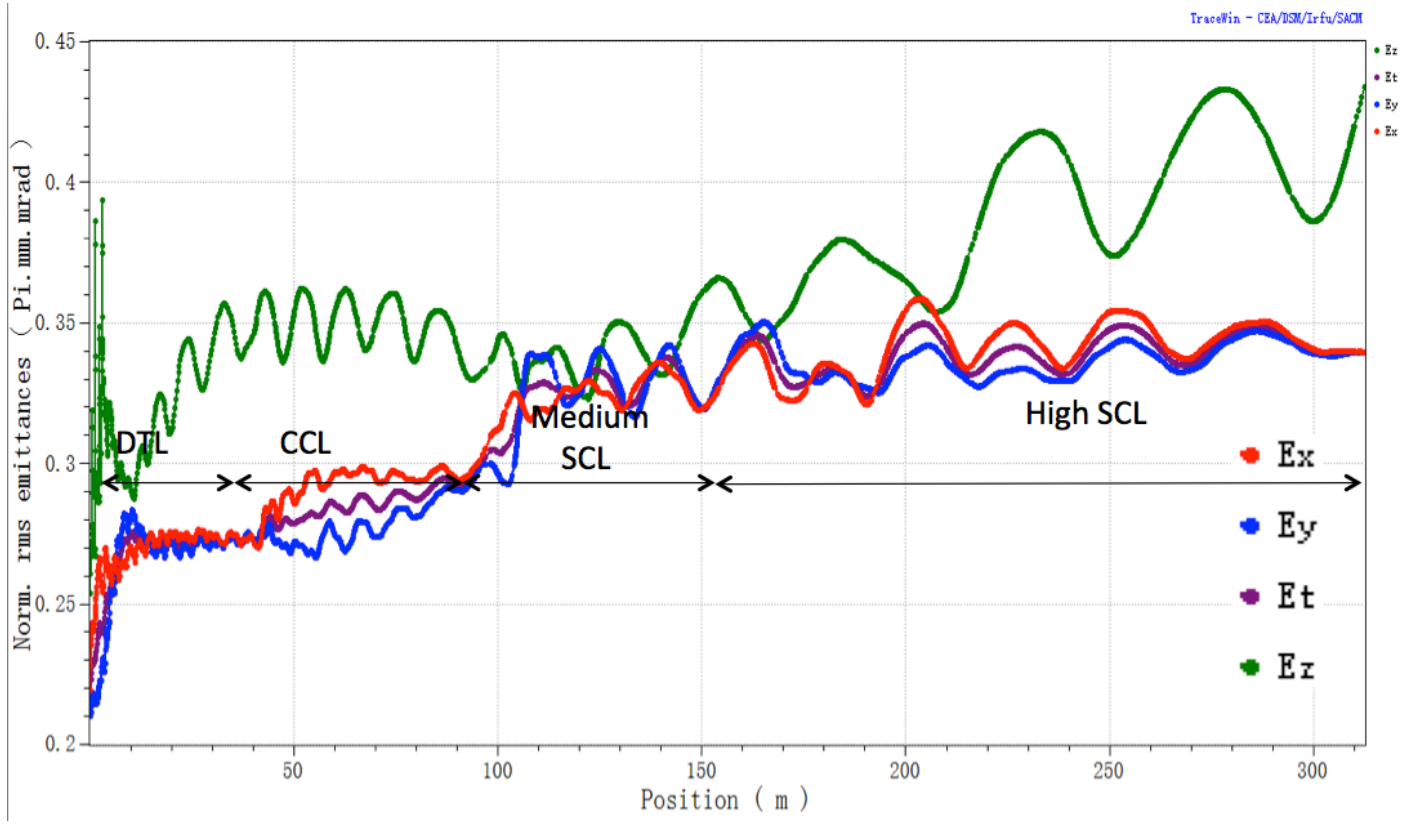

Figure 2.6. Simulated rms emittance growth in the STS linac.

\subsubsection{HEBT simulations}

The beam distribution from the linac simulation was used as a starting point for a separate particle-tracking simulation, using the ORBIT particle tracking code [4], of the high-energy beam transport line (HEBT) ending at the primary stripper foil in the ring injection. The results are in good agreement with expectations and are similar to the SNS baseline case. Figure 2.7 shows one of the resulting plots. The beam distribution at the foil is suitable for charge-exchange injection into the accumulator ring at the stripper foil.

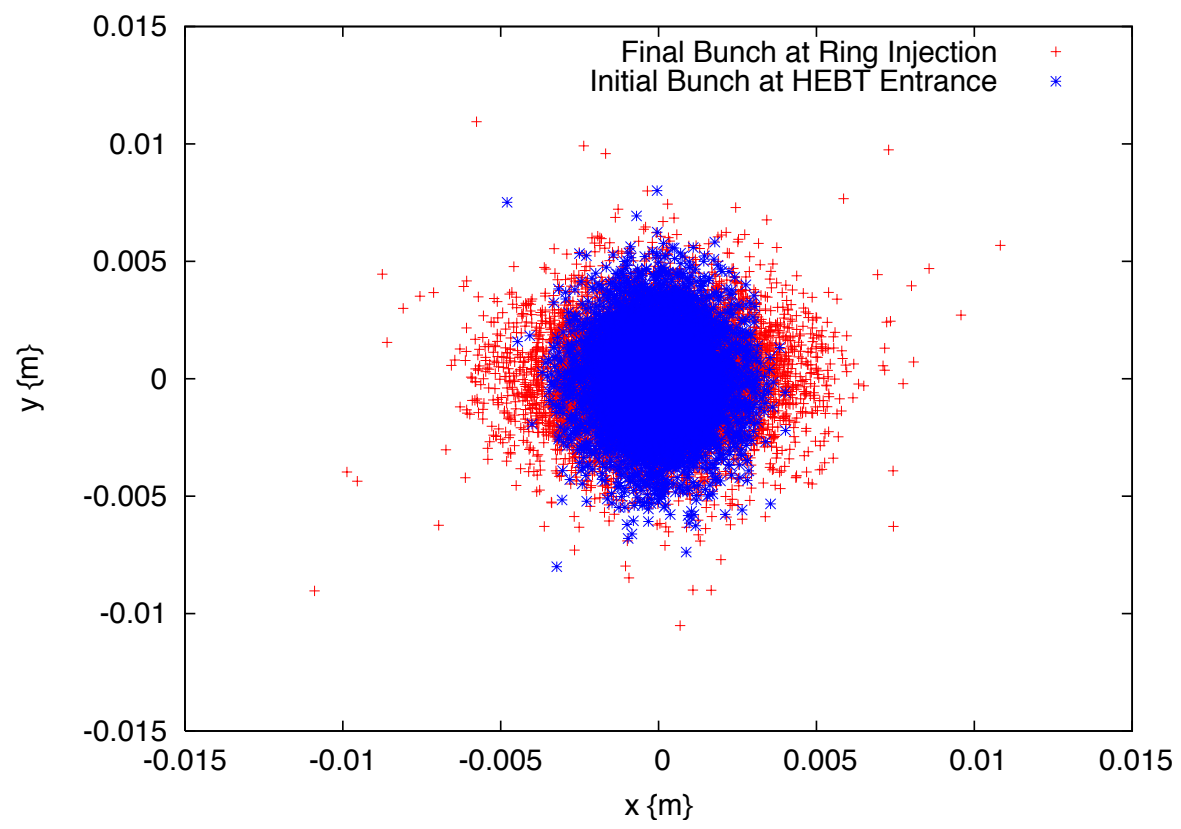

Figure 2.7. Beam distribution at the HEBT entrance (blue) and at the primary stripper foil in the ring injection area (red). 


\subsubsection{Ring and Ring-to-Target Beam Transport Simulations}

The ring simulation assumes the original SNS design input beam distribution at the stripper foil. This is acceptable because the STS HEBT simulation mentioned in Section 2.2.1 produces essentially the same distribution, and because the input distribution represents a small paintbrush stroke into the much larger ring aperture that is filled by the injection painting process. The simulation shows that beam delivery to the FTS will be essentially the same before and after the PPU upgrade. Figures 2.8 and 2.9 show the resultant beam distributions at the proton beam window upstream of the first target and at the face of the first target, respectively. No beam loss of concern is seen in these simulations, and the qualitative features are similar to the existing beam profiles on the target. Of course the peak density is higher owing to the increased number of protons striking the target. For example, the $2.0 \mathrm{MW}, 50 \mathrm{~Hz}$ case shown in Figure 2.9 has a peak density of $220 \mathrm{~mA} / \mathrm{m}^{2}$, which is $22 \%$ higher than the $180 \mathrm{~mA} / \mathrm{m}^{2}$ design case for $1.4 \mathrm{MW}$, $60 \mathrm{~Hz}$. The $2.0,50 \mathrm{~Hz}$ case has $28 \%$ more protons per pulse. The scaling is not exact because of small differences in the beam profiles.

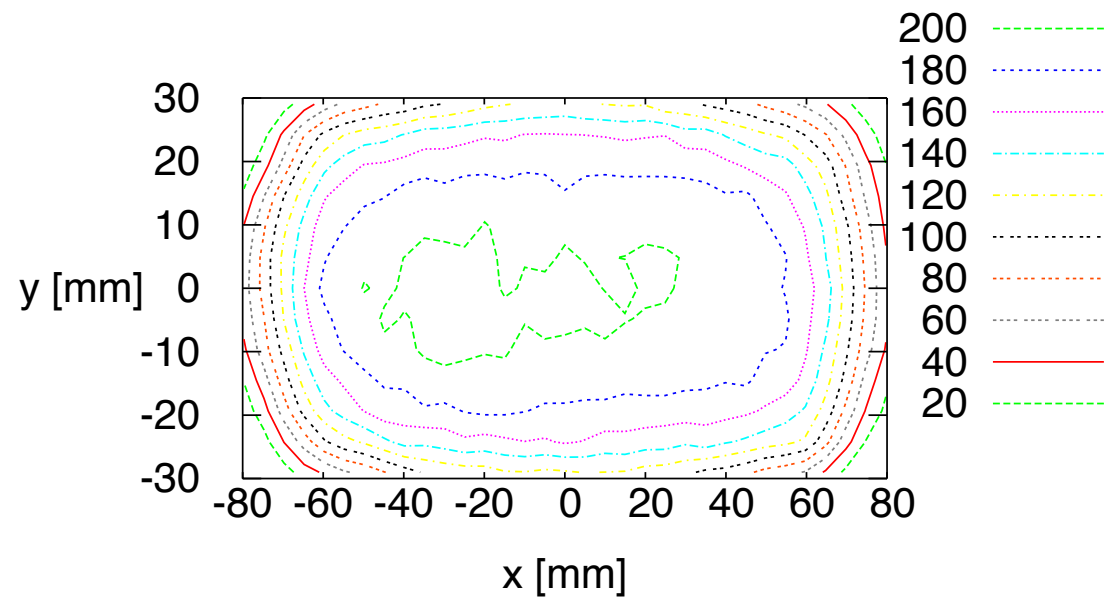

Figure 2.8. Beam distribution at the proton beam window upstream of the first target. The plot contours show the $\mathrm{mA} / \mathrm{m}^{2}$ value assuming a beam power of $2.0 \mathrm{MW}$ at $50 \mathrm{~Hz}$. The beam distribution for the $2.0 \mathrm{MW}, 60 \mathrm{~Hz}$ case is practically identical.

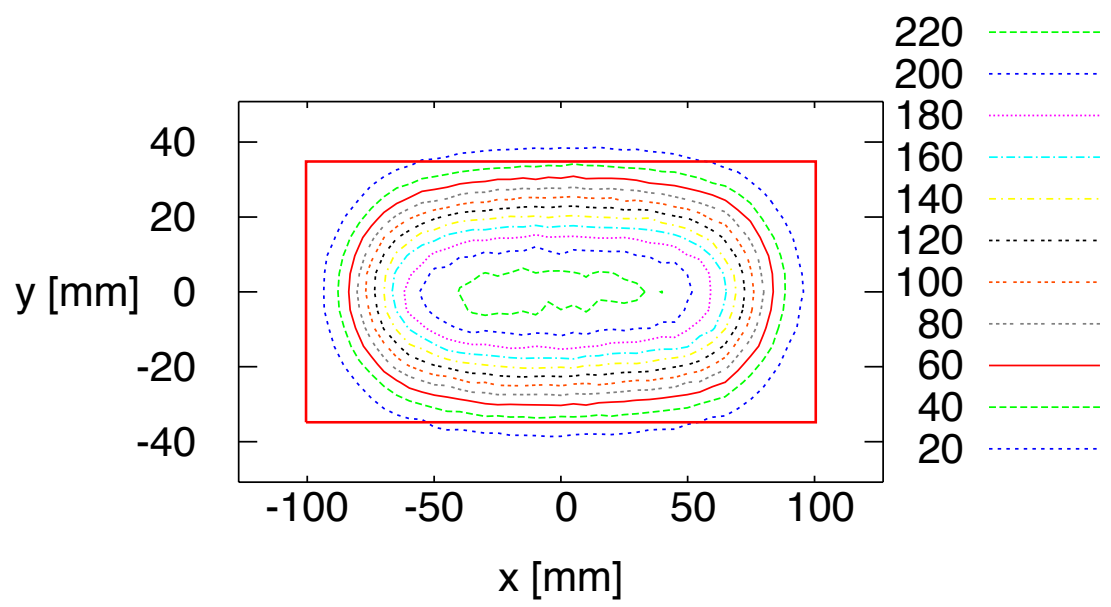

Figure 2.9. Beam distribution at the first target. The plot contours show the $\mathrm{mA} / \mathrm{m}^{2}$ value assuming a beam power of $2.0 \mathrm{MW}$ at $50 \mathrm{~Hz}$. The beam distribution for the $2.0 \mathrm{MW}, 60 \mathrm{~Hz}$ case is practically identical. 


\subsubsection{Space charge effects}

The purpose of this section is to assess the impact of collective effects on beam accumulation and transport at the proposed higher intensities for the PPU. Because the allowable losses in SNS are extremely small, the accumulator ring was constructed with a large aperture, and the beam pipe was coated with titanium nitride to reduce electron multipacting. The primary collective phenomena that could contribute to losses in the SNS ring are space charge, and extraction kicker impedances or electron clouddriven instabilities. In present operations, none of these phenomena contributes significantly to beam loss. Losses in the SNS ring at this time are dominated by beam scattering from the primary stripper foil [5]. However, it is necessary to assess the impact of collective effects as operation at still higher intensities is considered.

In the longitudinal direction, it is operationally possible to maintain a clean gap for beam extraction to the target with significantly less RF power than is called for in the original SNS design. From this fact, we can infer that longitudinal space charge and impedance effects will be mild. This is consistent with simulations performed during the SNS design that showed a wide margin of stability in the longitudinal plane [6]. In the transverse plane, space charge effects were observed to smooth the beam profiles and to introduce some coupling between the horizontal and vertical directions, but these had no effect on losses or on the ability to provide the required beam footprint at the target. In one controlled experiment, it was necessary to go to great lengths to induce instability because of the impedance of the extraction kickers. Simulations matched the observed growth rate of this instability under the conditions of the experiment [7], and similar simulations showed a wide margin of stability under normal operating conditions. The most likely cause of instabilities at high intensity may be electron cloud phenomena. In anticipation of this occurrence, the beam pipe in the ring was coated with titanium nitride to reduce electron multipacting. Even so, there are indications in the broadband transverse spectrum that the SNS ring beam may be at the onset of the electron cloud instability under present operating conditions. However, at present, no losses can be attributed to electron cloud activity.

It is possible to estimate analytically the effects of increased beam energy and intensity on the forces due to space charge and to known impedances. Table 2.5 presents such an estimate for four sets of parameters: present SNS production, the original design, the beam to the FTS with upgraded energy and intensity, and the beam to the STS with upgraded energy and intensity. The latter two cases use the parameters given in Plum et al. [8]. In all cases, the beam distribution sizes and shapes are considered to be the same, and the changes in impedance due to the altered ring and betatron frequencies are neglected. The scaling of the space charge and impedance forces is calculated as functions of beam energy and intensity only. However, an additional factor of $8 / 7$ is included in the impedance-induced forces for the upgrade scenarios, because the number of extraction kickers will increase by 2, from 14 to 16, for the upgrade. The quantities used to compare the effects of the forces under the four scenarios are the induced energy changes for the longitudinal forces and the angular kicks for the transverse forces. The strengths of the forces for all cases are normalized to those for the present production parameters. Under the stated assumptions, the strengths of all forces increase linearly with increasing beam intensity, as measured by number of injected protons. Except for the longitudinal impedance, this increase with intensity is offset by decreasing strength with increasing energy, as a result of relativistic effects and increasing beam stiffness.

Table 2.5 shows that, because of these offsetting effects, neither longitudinal nor transverse space charge forces should pose a problem under upgraded operational scenarios. The longitudinal space charge force barely increases with the PPU upgrade parameters, and the transverse space charge force actually decreases. The forces associated with the extraction kicker impedance do increase, especially for the PPU parameters. Previous simulations [7, 9] showed there is a wide margin of stability - a factor of at least five in the longitudinal direction and more than two in the transverse direction-with respect to the extraction kicker impedance. Given these results and the values in Table 2.5, we anticipate that operation 
at the upgrade parameters should be stable with respect to the space charge and impedance forces. To further support those expectations, we have performed simulations of the first and second target upgrade scenarios with the ORBIT code, which indicate stable operation with upgraded beam parameters.

Table 2.5. Comparison of collective forces in SNS for four operating scenarios: present production, the original design, first target with an intensity upgrade, and second target with intensity upgrade

\begin{tabular}{lcccc}
\hline \multicolumn{1}{c}{ Case } & Production & Design & $\begin{array}{c}\text { FTS at } \\
\mathbf{2} \mathbf{~ M W , ~ 6 0 ~ H z ~}\end{array}$ & $\begin{array}{c}\text { Full intensity } \\
\mathbf{2 . 8} \mathbf{~ M W , ~ 6 0 ~ H z}\end{array}$ \\
\hline Kinetic energy $(\mathrm{GeV})$ & 0.94 & 1.0 & 1.3 & 1.3 \\
Relativistic $\gamma$ & 2.002 & 2.066 & 2.386 & 2.386 \\
Relativistic $\beta$ & 0.866 & 0.875 & 0.908 & 0.908 \\
$\mathrm{~N}$ protons $\left(\times 10^{14}\right)$ & 1.55 & 1.5 & 1.61 & 2.241 \\
Longitudinal space charge $\left(\Delta \mathrm{E} \sim \mathrm{N} \times \gamma^{-2}\right)$ & 1.0 & 0.909 & 0.728 & 1.026 \\
Longitudinal impedance $(\Delta \mathrm{E} \sim \mathrm{N} \times \beta)$ & 1.0 & 0.977 & ${ }^{a} 1.24$ & ${ }^{a} 1.745$ \\
Transverse space charge & 1.0 & 0.863 & 0.556 & 0.784 \\
$\left(\Delta \theta \sim \mathrm{N} \times \gamma^{-3} \times \beta^{-2}\right)$ & & & & \\
Transverse impedance $\left(\Delta \theta \sim \mathrm{N} \times \gamma^{-1} \times \beta^{-1}\right)$ & 1.0 & 0.928 & ${ }^{a} 0.945$ & ${ }^{a} 1.333$ \\
\hline
\end{tabular}

${ }^{a}$ Impedance forces for upgrade scenarios multiplied by $8 / 7$ to account for two additional extraction kickers.

Simple analytic estimation is not possible for the effect of electron clouds on the accumulating proton beam. The specifics of the generation mechanisms, locations, and quantities of ambient electrons in the accelerator are not precisely known. It is generally accepted that ambient electrons are generated by beam collisions with the beam pipe (beam loss), by ionization of the imperfect vacuum by the beam, and by multipacting from existing electrons striking the beam pipe [10]. A possible additional mechanism in SNS is incomplete collection of stripped electrons at the injection point. The behavior of ambient electrons generated in the beam pipe will depend on their location. Electrons in a drift space, dipole field, or quadrupole field have different orbits and will respond to the beam in different ways. Little is known of the locations of electrons in the SNS ring, but it may be assumed that they are located in the vicinity of the highest beam losses, downstream of the injection point and in the collimation section.

At the SNS, steps have been taken that mitigate the electron generation mechanisms, although the primary motivation for some of these steps is to minimize activation from beam loss. Fractional beam losses are maintained at a very low level $\left(\sim 10^{-4}\right)$, and low beam loss corresponds to the generation of fewer electrons. The large beam pipe aperture ( 8 in. diameter) helps in loss minimization, and it places the location of electron multipacting further from the beam. A high vacuum of $10^{-8}$ torr is maintained in the ring, so electron generation by gas ionization is small. The geometry of the magnetic field in the vicinity of the stripper foil has been carefully designed and studied [11] to guide the stripped electrons to a collector. The walls of the SNS ring have been coated with titanium nitride for the specific purpose of reducing the secondary electron yield from multipacting. Additionally, a broadband feedback system has been developed to counter the effect of any observed electron cloud-induced instability [12].

Some simulations of the electron cloud dynamics in SNS have been performed $[13,14]$. According to Shishlo et al. [14], a linearized stability model and more detailed simulations are consistent with one another in predicting that, with $2 \times 10^{14}$ protons, SNS should be stable for first harmonic RF voltages exceeding $15 \mathrm{kV}$. The available first harmonic RF voltage in SNS is $40 \mathrm{kV}$. Overall, we anticipate that SNS can be operated stably with respect to electron clouds at the upgraded parameters. If electron cloud instabilities should arise, additional measures, such as broadband feedback stabilization and longitudinal profile tailoring, can be applied. 


\subsection{BEAM LOSS AND RESIDUAL ACTIVATION}

With the increase in beam power, there will be an associated increase in beam loss and residual activation [15].

\subsubsection{Residual Activation vs. Beam Energy}

The residual activation (dose rate) depends on the beam power lost and the beam energy, as shown in Figure 2.10. The functional dependence, assuming copper (other materials are similar), is

$$
\text { dose rate }=0.33 \cdot\left(\mathrm{E}^{-9}\right)^{1.8} / E,
$$

where $E$ is the beam energy in $\mathrm{MeV}$, and the dose rate is in $\mathrm{mrem} / \mathrm{h}$ at $30 \mathrm{~cm}$ after a 4 hour cooldown. The plot ends at $1.2 \mathrm{GeV}$, and we assume that is still approximately correct at $1.3 \mathrm{GeV}$.

For a given number of protons lost per second, and keeping everything the same except for the beam energy, the activation is then proportional to

$$
\text { dose rate } \propto(E-9)^{1.8}
$$

For a given number of protons lost per second at $1.3 \mathrm{GeV}$, the dose rate will be 1.61 times higher than at $1.0 \mathrm{GeV}$.

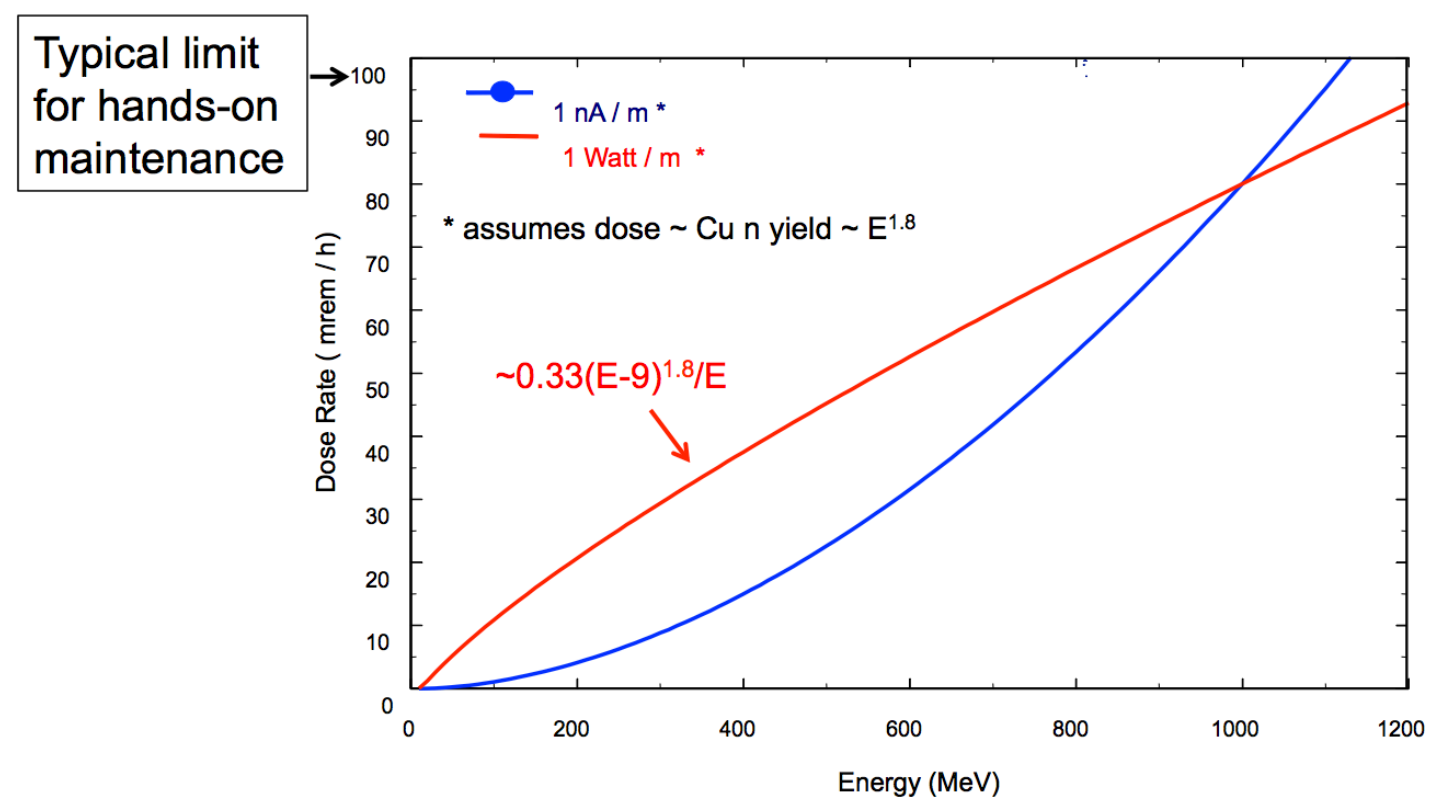

Figure 2.10. Activation as a function of beam energy [16].

\subsubsection{Beam Loss in the Warm Linac}

Beam loss in the warm linac is dominated by residual gas stripping and halo scraping (personal communication from A. Shishlo, ORNL, to M. Plum, ORNL, August 4, 2016). Intra-beam stripping is present but at levels much lower than the other mechanisms. To first order, we expect the beam loss to be proportional to the average beam current. The $2.8 \mathrm{MW}$ case has an average current that is 1.44 times higher than the 1.4 MW case, so we expect the warm linac beam loss to also be 1.44 times higher. 


\subsubsection{Beam Loss in the Superconducting Linac}

Beam loss in the SCL is dominated by intra-beam stripping [17]. The beam loss caused by this mechanism is proportional to the square of the beam density. Compared with the $1.4 \mathrm{MW}$ case, the 2.8 MW case will have a 25 us longer macropulse and 1.44 times higher average current, so the beam density will be 1.404 times higher. The beam loss is therefore expected to be 1.97 times higher.

In the downstream portion of the SCL, where the dummy section is now located, the activation levels could be more than 1.97 times higher, simply because of the beam energy exceeding $1.0 \mathrm{GeV}$ in this area. Therefore, at the end of the SCL, the dose rate could increase by a factor of 1.97.1.61 $=3.17$ (see Section 2.3.1 for the 1.61 factor discussion). However, note that, in practice, the SCL activation levels do not seem to depend significantly on the beam energy (perhaps because of adiabatic damping?), so historical evidence provides a counter-argument for the extra factor of 1.61 .

\subsubsection{Beam Loss in the Ring Injection Area}

The ring injection area has by far the most radioactivation in the accelerator, and the dominant cause is large-angle Coulomb and nuclear scattering in the stripper foil. At the Los Alamos National Laboratory Proton Storage Ring, nuclear and large-angle Coulomb scattering contribute nearly equal amounts to the beam loss $(\sim 35 \%$ due to large-angle Coulomb vs. $\sim 30 \%$ due to nuclear $)$ [18]. In the following discussion, we will assume equal contributions. At SNS the excited-state H0 losses are expected to be very small as a result of placing the stripper foil inside the chicane magnet.

\subsubsection{Coulomb scattering}

The probability of a large-angle Coulomb scattering event causing beam loss is [18]

$$
\mathrm{P}=5.674 \cdot 10^{-8}\left\{\mathrm{~cm}^{2}\right\}\left(\frac{\mathrm{Z}}{\gamma \beta^{2}}\right)^{2}\left(\frac{\rho \mathrm{t}}{\mathrm{A}}\right)\left[\frac{1}{\theta_{\mathrm{xl}} \theta_{\mathrm{yl}}}+\frac{1}{\theta_{\mathrm{xl}}^{2}} \tan ^{-1}\left(\frac{\theta_{\mathrm{yl}}}{\theta_{\mathrm{xl}}}\right)+\frac{1}{\theta_{\mathrm{yl}}^{2}} \tan ^{-1}\left(\frac{\theta_{\mathrm{xl}}}{\theta_{\mathrm{yl}}}\right)\right],
$$

where $\rho t$ is the effective foil thickness, $Z$ and $A$ are the atomic number and atomic mass of the foil material (carbon), $\beta$ and $\gamma$ are the relativistic factors, and $\theta_{x l}$ and $\theta_{y l}$ are the limiting angles, obtained from the limiting apertures. The foil thickness at $1.3 \mathrm{GeV}$ will be $8 \%$ thicker than at $1.0 \mathrm{GeV}$.

Based on Eq. (2.3), we see that the scattering probability is directly proportional to the foil thickness $\rho$. From this equation, we see that the scattering probability scales inversely with $\gamma^{2} \beta^{4}$. Per injected proton, the number of beam particles lost will therefore be lower at $1.3 \mathrm{GeV}$ than at $1.0 \mathrm{GeV}$ because the factor of 1.91 decrease in scattering probability more than compensates for the $8 \%$ increase in foil thickness.

For a given number of injected protons, keeping everything the same except for the beam energy and the foil thickness, the activation is then proportional to

$$
\text { dose rate } \propto\left(\frac{1}{\gamma \beta^{2}}\right)^{2}(\rho t)(E-9)^{1.8} \text {. }
$$

For a given number of injected protons, the dose rate at $1.3 \mathrm{GeV}$ is $12 \%$ higher than at $1.0 \mathrm{GeV}$.

To compare $1.0 \mathrm{GeV}, 1.4 \mathrm{MW}$ with $1.3 \mathrm{GeV}, 2.8 \mathrm{MW}$, note that there are $2.24 / 1.55=1.44$ times more injected protons (stored proton intensity ratio) for the $2.8 \mathrm{MW}$ case, so the dose rate for the $2.8 \mathrm{MW}$ case is 1.63 times the dose rate for the $1.4 \mathrm{MW}$ case. 


\subsubsection{Nuclear scattering}

Until detailed simulations can be performed, we estimate the nuclear scattering effect by assuming the proton-carbon cross section is proportional to the sum of the pp and pn cross sections. Based on data from the Lawrence Berkeley Laboratory Particle Data Group, the sums of the pp and pn total cross sections are nearly the same at 1.696 and $2.386 \mathrm{GeV} / \mathrm{c}$ (corresponding to 1.0 and $1.3 \mathrm{GeV}$ beam energies). We conclude that the probability of beam loss due to nuclear scattering is approximately equal for the 1.0 and $1.3 \mathrm{GeV}$ cases (for a given foil thickness). Following the arguments in Section 2.3.4.1,

$$
\text { dose rate } \propto(\rho t)(E-9)^{1.8}
$$

Including the greater number of injected protons, the dose rate due to nuclear scattering at $2.8 \mathrm{MW}$ is expected to be 2.51 times greater than at $1.4 \mathrm{MW}$.

\subsubsection{Both large-angle Coulomb and nuclear scattering}

We assume that large-angle Coulomb and nuclear scattering contribute equally to the total beam loss because of stripper foil interactions. The dose rate then scales as

$$
\text { dose rate } \propto\left[1+\left(\frac{1}{\gamma \beta^{2}}\right)^{2}\right](\rho t)(E-9)^{1.8} .
$$

For a given number of injected protons, keeping everything the same except for the beam energy and the foil thickness, the dose rate at $1.3 \mathrm{GeV}$ is expected to be 1.56 times higher than at $1.0 \mathrm{GeV}$. Adding in the factor of 1.44 times more protons for the $2.8 \mathrm{MW}$ case compared with the $1.4 \mathrm{MW}$ case gives an estimated dose rate 2.26 times higher.

\subsubsection{Beam loss in the ring collimation section}

In addition to large-angle Coulomb and nuclear scattering in the stripper foil, there is small-angle scattering. This type of scattering causes a slow growth in the beam tails that primarily causes beam loss in the collimation section.

Based on the work of Hinterberger, Mayer-Kuckuk, and Prashun [19], the rms scattering angle of a proton beam passing through a very thin film is

$$
\theta_{r m s}=\frac{14.1 \mathrm{MeV}}{p \beta c} \sqrt{\left(x / x_{\text {rad }}\right)}
$$

where $p$ is the proton beam momentum, $\beta c$ is the proton velocity, $x$ is the stripper foil thickness, and $x_{\text {rad }}$ for carbon is $42.7 \mathrm{~g} / \mathrm{cm}^{2}$. (This is similar to Eq. [2.6], but without the logarithmic term). For our case, at $1.0 \mathrm{GeV}$ and for a $0.385 \mathrm{mg} / \mathrm{cm}^{2}$ thick foil, $\theta_{r m s}=2.2 \times 10^{-5} \mathrm{rad}$; and at $1.3 \mathrm{GeV}$ and a $0.416 \mathrm{mg} / \mathrm{cm}^{2}$ thick foil ( $8 \%$ thicker), $\theta_{r m s}=2.0 \times 10^{-5} \mathrm{rad}$. The rms scattering angle is $9 \%$ lower at $1.3 \mathrm{GeV}$, but the beam energy is higher.

It is not exactly correct to say that the beam loss scales with the rms scattering angle, but we can use it as a rough estimate. We assume that

$$
\text { dose rate } \propto \theta_{\text {rms }}(E-9)^{1.8}
$$

and find that for a given number of injected protons, keeping everything the same except for the beam energy and the foil thickness, the dose rate at $1.3 \mathrm{GeV}$ is expected to be 1.46 times higher than at 
$1.0 \mathrm{GeV}$. Adding in the factor of 1.44 times more protons for the $2.8 \mathrm{MW}$ case compared with the 1.4 MW case gives an estimated dose rate 2.11 times higher.

\subsubsection{Beam Loss in the HEBT and Ring-to-Target Beam Transport}

The fractional beam loss in the HEBT and ring-to-target beam transport (RTBT) should be about the same at 1.0 and $1.3 \mathrm{GeV}$. The space charge effects are small at these energies. We therefore expect the number of particles lost will scale with the total number of particles. The dose rate will be higher because of the higher beam energy.

For a given number of protons, keeping everything the same except for the beam energy, the dose rate at $1.3 \mathrm{GeV}$ is expected to be 1.61 times higher than at $1.0 \mathrm{GeV}$. Including the factor of 1.44 times more protons for the $2.8 \mathrm{MW}$ case compared with the $1.4 \mathrm{MW}$ case gives an estimated dose rate 2.33 times higher.

\subsubsection{Activation}

Figure 2.11 shows recent dose rate data taken after $1.3 \mathrm{MW}$ operation. The expected increase in dose rate at $1.0 \mathrm{GeV}, 1.4 \mathrm{MW}$ is a factor of $1.4 / 1.3=8 \%$ times greater. The expected dose rate increase from 1.4 to 2.8 MW is summarized in Table 2.6.

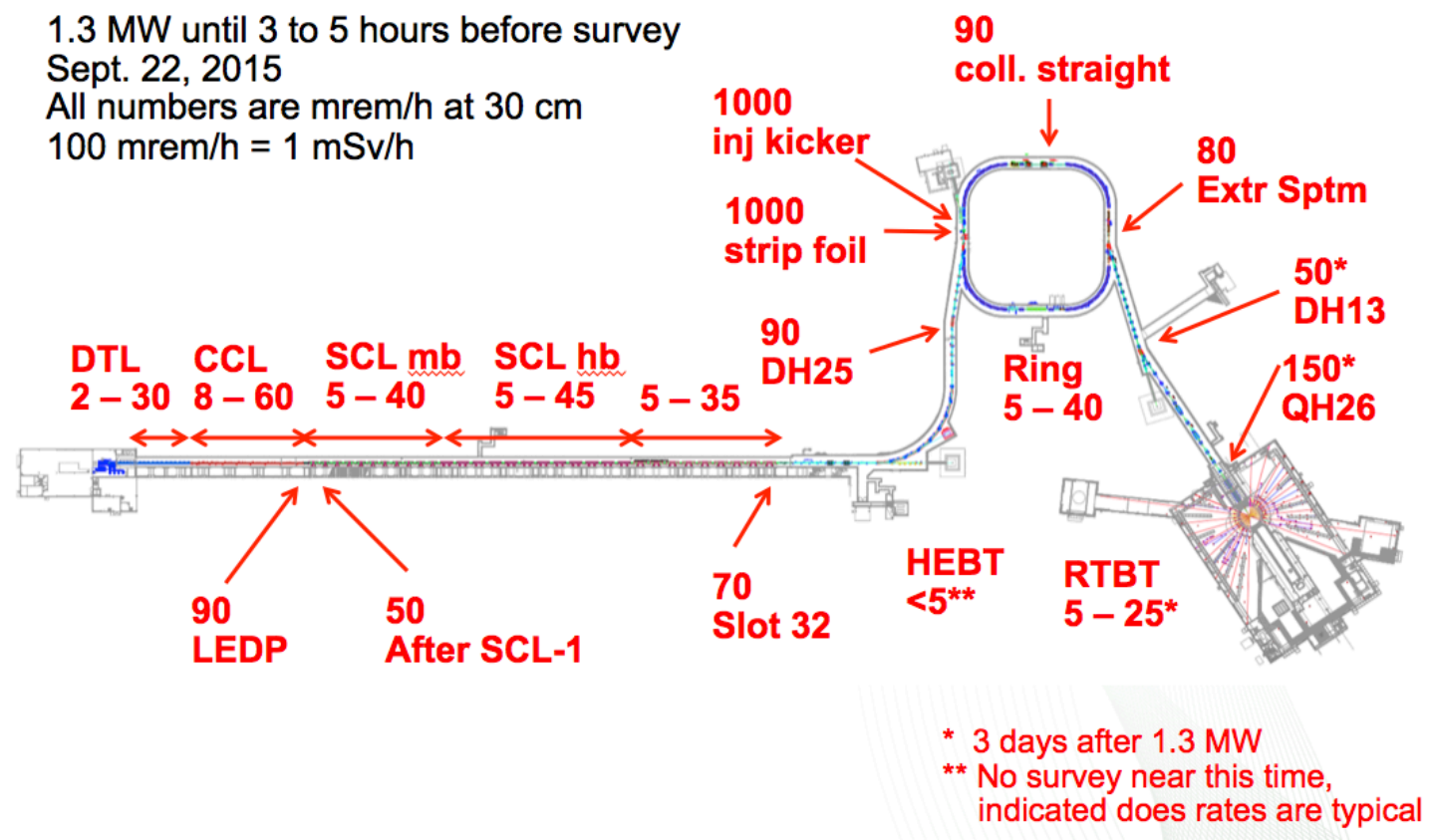

Figure 2.11. Recent dose rate data measured on September 22, 2015, after 1.3 MW operation. 
Table 2.6. Expected increase in dose rates from 1.4 to

2.8 MW operation

\begin{tabular}{lc}
\hline \multicolumn{1}{c}{ Location in accelerator } & Dose rate increase factor \\
\hline Warm linac & 1.44 \\
SCL (up to $1.0 \mathrm{GeV}$ section) & 1.97 \\
SCL (1.3 GeV section) & $1.97-3.17$ \\
HEBT & 2.33 \\
Ring injection & 2.26 \\
Ring collimation & 2.11 \\
RTBT & 2.33 \\
\hline
\end{tabular}

\subsection{HEBT ARC BEAM LOSS VS BEAM ENERGY}

The HEBT was designed to transport $\mathrm{H}^{-}$beam at energies up to $1.3 \mathrm{GeV}$. The discussion in this section details the consequences of increasing the beam energy beyond $1.3 \mathrm{GeV}$.

Magnetic stripping [20] as a function of beam energy is

$$
\frac{d f}{d s}=\frac{B(s)}{A 1} e^{-A 2 / \beta \gamma c B(s)},
$$

where $A 1=2.47 \mathrm{E}-6 \mathrm{~V} \mathrm{sec} / \mathrm{m}, A 2=4.49 \mathrm{E} 9 \mathrm{~V} / \mathrm{m}, B(s)=$ magnetic field, and $\beta, \gamma, c$ are the usual relativistic factors.

The HEBT arc has eight dipoles that bend the beam by $90^{\circ}$. The magnetic length of each dipole is 5.432 $\mathrm{m}$, and the total magnetic length is $43.456 \mathrm{~m}$. To bend the beam through an angle $\theta$ requires a B-field,

$$
B=\frac{\theta(B \rho)}{L},
$$

where $(B \rho)$ is the beam rigidity, $B \rho=3.1297 \beta \gamma$ for proton beams, and $L$ is the magnetic length. Then $d f / d s$ can be expressed in terms of just $\beta$ and $\gamma$, and the fractional loss per meter in the HEBT arc can be expressed in terms of just the beam energy. A plot of the result is shown in Figure 2.12.

At a beam energy of $1.3 \mathrm{GeV}$, the fractional loss per meter is $5.5 \mathrm{E}-8$. For a beam power of $2.8 \mathrm{MW}$, this corresponds to $0.154 \mathrm{~W} / \mathrm{m}$. The rule-of-thumb upper limit is $1 \mathrm{~W} / \mathrm{m}$ for the total beam loss, so beam operation at $1.3 \mathrm{GeV}, 2.8 \mathrm{MW}$ leaves only a small margin for the other beam loss mechanisms. If the beam energy were $1.36 \mathrm{GeV}$, the magnetic stripping losses would be $1 \mathrm{~W} / \mathrm{m}$ and the total losses would certainly exceed $1 \mathrm{~W} / \mathrm{m}$. The $1.3 \mathrm{GeV}$ design energy of the HEBT therefore represents a fairly hard upper limit. 


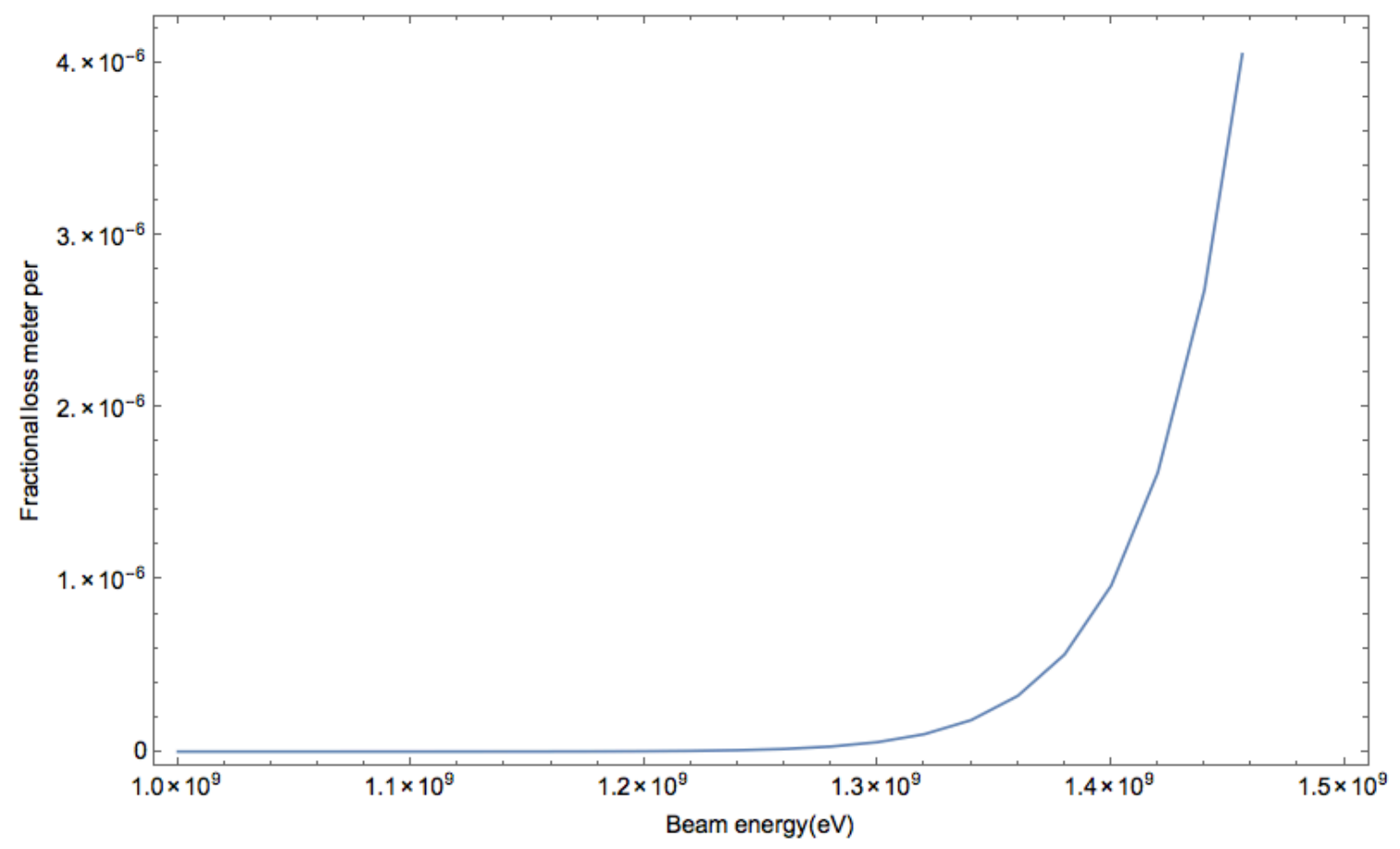

Figure 2.12. Plot of the fractional loss per meter in the HEBT arc vs. beam energy.

\subsection{ASSUMPTIONS}

In this chapter, we have assumed that medium beta cavity $11 \mathrm{~b}$ will be repaired in time for the final commissioning of the upgraded linac.

\subsection{REFERENCES}

[1] S. Henderson et al., "Status of the SNS Beam Power Upgrade Project," pp. 345-347 in Proceedings of EPAC 2006, Edinburgh, Scotland (2006).

http://accelconf.web.cern.ch/AccelConf/e06/PAPERS/MOPCH129.PDF.

[2] J. Lyon and S. Cousineau, "Study of simulations of variable chopper pattern widths," summer intern presentation, 2015.

[3] TraceWin, The Service of Accelerators, Cryogenics, and Magnetism. Available at http://irfu.cea.fr/Sacm/logiciels/index3.php. Accessed September 2016.

[4] J. A. Holmes, S. Cousineau, V. V. Danilov, et al., "ORBIT: Beam dynamics calculations for highintensity rings," ICFA Beam Dynamics Newsletter 30, pp. 100-107, International Committee for Future Accelerators (April 2003).

[5] J. A. Holmes and M. A. Plum, "Beam loss due to foil scattering in the SNS accumulator ring," pp. 254-258 in Proceedings of the ICFA High Brightness Workshop HB2012, Beijing, September 1721, 2012.

[6] K. Woody, J. A. Holmes, V. Danilov, and J. D. Galambos, "Longitudinal impedance simulations in ORBIT: Benchmarking and application to the SNS extraction kicker," pp. 2057-2059 in

Proceedings of the 2001 Particle Accelerator Conference (PAC 2001), Chicago, June 18-22, 2001. 
[7] J. A. Holmes, S. Cousineau, V. Danilov, and L. Jain, "Comparison between measurements, simulations, and theoretical predictions of the extraction kicker transverse dipole instability in SNS," Phys. Rev. Special Topics-Accelerators and Beams 14, 074401 (2011).

[8] M. A. Plum, J. Galambos, and S.-H. Kim, "Accelerator systems modifications for a Second Target Station at the Oak Ridge Spallation Neutron Source," in Proceedings of the International Particle Accelerator Conference (IPAC14), Dresden, Germany, June 15-20, 2014.

[9] J. A. Holmes, V. Danilov, and L. Jain," Transverse stability studies of the SNS Ring," in Proceedings of the Particle Accelerator Conference (PAC05), Knoxville, Tennessee, May 16-20, 2005.

[10] M. A. Furman and M. Pivi, "Probabilistic model for the simulation of secondary electron emission," Phys. Rev. Special Topics-Accelerators and Beams 5, 124404 (2002).

[11] S. Cousineau, J. A. Holmes, M. A. Plum, and W. Lu, "Dynamics of uncaught foil-stripped electrons in the Oak Ridge Spallation Neutron Source accumulator ring," Phys. Rev. Special TopicsAccelerators and Beams 14, 064001 (2011).

[12] C. Deibele, S. Assadi, M. Schulte, and Z. Xie, "Status and implementation of a wideband feedback system for E-P instabilities in the SNS," p. 462 in Proceedings of the High Brightness (HB2008) Conference, Nashville, Tennessee, August 25-29, 2008.

[13] M. Blaskiewicz, M. A. Furman, M. Pivi, and R. J. Macek, "Electron cloud instabilities in the Proton Storage Ring and Spallation Neutron Source," Phys. Rev. Special Topics-Accelerators and Beams 6, 2003, 014203.

[14] A. Shishlo, S. Cousineau, V. Danilov, et al., "Electron cloud self-consistent simulations for the SNS ring," pp. 2832-2834 in Proceedings of the European Particle Accelerator Conference (EPAC06), Edinburgh, Scotland, June 26-30, 2006.

[15] M. Plum, Beam Loss and Activation for PPU-STS, ORNL/LTR-2016/375, Oak Ridge National Laboratory, August 8, 2016.

[16] J. Galambos, “SNS Beam Loss, Activation, and Collimation," Snowmass 2001, Snowmass Village, June 30-July 21, 2001, July 7, 2001.

[17] A. Shishlo et al., "First observation of intrabeam stripping of negative hydrogen in a superconducting linear accelerator," Phys. Rev. Lett. 108, 114801 (2012).

[18] R. Macek, "PSR beam losses at injection," in High Intensity and High Brightness Hadron Beams: 33rd ICFA Advanced Beam Dynamics Workshop, ICFA-HB2004, eds. I. Hoffmann, J.-M. Lagniel, and R. W. Hasse, Bensheim, Germany, October 18-22, 2004,AIP Conference Proceedings, June 8, 2005 .

[19] F. Hinterberger, T. Mayer-Kuckuk, and D. Prashun, "Monte Carlo simulations of thin internal targets in intermediate energy proton storage rings," Nucl. Inst. Meth. A275, 239-245, (1989).

[20] A. Jason, D. W. Hudgings, and O. B. VanDyck, "Neutralization of $\mathrm{H}^{-}$beams by magnetic stripping," IEEE Trans. Nucl. Sci. NS-28(3) 2704 (June 1981). 



\section{SUPERCONDUCTING LINAC}

Seven cryomodules will be installed in the reserved space at the end of the linac tunnel to produce a linac output energy of $1.3 \mathrm{GeV}$ with an energy margin specified in Section 2.1.

The optimum geometric beta of the cavities for the PPU is higher than that of the original high-beta cavities; but the module length and, accordingly, the cavity geometric beta will be kept the same for economic reasons. All helium transfer lines were previously installed during the SNS project with bayonets for the high-beta cryomodules. Waveguide penetrations from the klystron gallery to the linac tunnel were also previously installed.

Some changes that do not require modification of the overall layout will be made based on the lessons learned from experience over the last 10 years and on the pressure-vessel compliance issue. Table 3.1 summarizes the design changes between the original SNS high-beta cryomodule and the high-beta cryomodule for the PPU. Some details for the changes are described in the following subsections.

Table 3.1. Design parameter changes

\begin{tabular}{lll}
\hline \multicolumn{1}{c}{ Parameters } & \multicolumn{1}{c}{$\begin{array}{c}\text { Original SNS high-beta } \\
\text { cryomodule }\end{array}$} & \multicolumn{1}{c}{ PPU high-beta cryomodule } \\
\hline $\begin{array}{l}\mathrm{E}_{\mathrm{acc}}\left(=\mathrm{E}_{\mathrm{o}} \mathrm{T}_{\mathrm{g}}, \mathrm{T}_{\mathrm{g}} \text { : Transit time factor at }\right. \\
\beta=0.81)(\mathrm{MV} / \mathrm{m})\end{array}$ & 15.8 & 16.0 \\
$\begin{array}{l}\text { Fundamental power coupler }(\mathrm{FPC}) \\
\text { rating, peak and average }(\mathrm{kW})\end{array}$ & 550,50 & 700,65 \\
$\begin{array}{l}\text { External Q of FPC, Qex } \\
\text { Material of cavity }\end{array}$ & $\begin{array}{l}7 \times 10^{5}( \pm 20 \%), \text { fixed type } \\
\text { High RRR niobium (RRR }>250) \text { for } \\
\text { cells, reactor-grade niobium for end } \\
\text { groups }\end{array}$ & $\begin{array}{l}\text { High RRR niobium (RRR }>250) \\
\text { for both cells and end groups }\end{array}$ \\
Higher-order mode couplers per cavity & $\begin{array}{l}\text { Two (one at each end group) } \\
\text { One mechanical tune, one fast piezo } \\
\text { tuner }\end{array}$ & $\begin{array}{l}\text { None } \\
1 \text { mechanical tuner (no fast piezo } \\
\text { tuner) }\end{array}$ \\
Tuner & Good engineering practice & Code stamp required \\
\hline Pressure vessel & &
\end{tabular}

$\mathrm{RRR}=$ residual resistivity ratio

\subsection{SRF CAVITY}

The cavity RF parameters for the PPU are the same as for the original ones. Table 3.2 shows the common parameters between the original and PPU cavities. 
Table 3.2. Major parameters of the SNS high-beta cavity

\begin{tabular}{lc}
\hline Frequency $(\mathrm{MHz})$ & 805 \\
Type & Elliptical \\
Operating mode & pi \\
Geometric beta & 0.81 \\
Equivalent cavity length $(\mathrm{mm})$ & 906 \\
Bore radius $(\mathrm{mm})$ & 48.8 \\
Inter-cell coupling $(\%)$ & 1.6 \\
$\mathrm{r} / \mathrm{Q}$ at geometric beta at $\beta=0.81(\mathrm{ohm})$ & 483 \\
Epeak/Eacc & 2.2 \\
Beak $/$ Eacc $(\mathrm{mT} / \mathrm{MV} / \mathrm{m})$ & 4.75 \\
Wall thickness $(\mathrm{mm})$ & $4(\sim 3.8$ after chemical processing) \\
Operating temperature & $2 \mathrm{~K}$ \\
Qo & $>5 \times 10^{9}$ at $2 \mathrm{~K}$ \\
Number of cells per cavity & 6 \\
Stiffener & Yes $($ at $\mathrm{r}=80 \mathrm{~mm})$ \\
Fundamental power coupler per cavity & 1 \\
\hline
\end{tabular}

\subsubsection{Accelerating Gradient}

The accelerating gradients of the PPU cavities, shown in Section 2.1, are based on the results from the spare high-beta cryomodule and the R\&D for performance improvements.

New cavities for the PPU. A high-beta spare cryomodule was developed in-house and has been installed in the tunnel for operation since summer 2012. All four cavities in the spare cryomodule were commissioned at $17 \mathrm{MV} / \mathrm{m}$ or higher at the full duty factor. Only one cavity shows minor x-rays starting from $15.5 \mathrm{MV} / \mathrm{m}$, and all four cavities have been running at $16 \mathrm{MV} / \mathrm{m}$ for the production run [1,2]. The available RF power limits the operating gradients to $16 \mathrm{MV} / \mathrm{m}$ for these cavities. The performance of the original SNS cavities in the linac is mainly limited by field emission and multipacting, resulting in lower operating gradients at $13.5 \mathrm{MV} / \mathrm{m}$ on average. Improvement of the cavity performance for the spare cryomodule came from changing the cavity chemical processing recipe from buffered chemical processing $(\mathrm{BCP})$ to electropolishing $(\mathrm{EP})$. EP produces a smoother surface finish and has typically shown higher operational gradients and higher field emission onset. Another identified limitation of all original SNS cavities is that the end group surfaces are very rough as a result of high-aspect-ratio deep drawing and the additional heat treatments. These end groups were fabricated from reactor-grade niobium, rather than high-purity or high-residual resistivity ratio (RRR) material, in an attempt to reduce the material costs of the cavities. The reactor-grade material was then heat-treated in a vacuum furnace to improve its thermal conductivity, but the treatment increased the niobium grain size in the process. The increased grain size, coupled with BCP, resulted in preferential etching of the grain edges, creating a very rough surface finish on all original SNS cavities. This rough surface on the end groups is difficult to clean and has many sharp edges at the grain boundaries. It could also produce trapped volumes for particulates and gases, consequently increasing the secondary emission yield and field emission. The EP process that was applied to the spare cryomodule cavities improved the surfaces of both the cells and the end groups and allowed for better cleaning of all surfaces following the chemical processing. The cavity performance observed in the high-beta spare cryomodule offers high confidence that the PPU cryomodules will meet the gradient specification by using EP instead of BCP for the final chemistry and by improving the cavity end groups. 
Original SNS cavities in the tunnel. The original cavities show electron loading from field emission and multipacting below the design accelerating gradients, which are the main limiting factor. In addition, as the repetition rate was increased, more severe collective effects between cavities were observed-i.e., operation of one cavity affecting the performance of neighboring cavities. The severity of the collective effects is determined not only by the amplitude of the accelerating gradients in the cavities of a cryomodule, but also by their relative RF phases. This is so because both affect the trajectories of the electrons, the electron energies, and the locations at which the electrons impact on the cavity surfaces. As a result of the collective effects, cavities became thermally unstable at lower gradients than they did during lower-repetition-rate operation, thus limiting the final linac output energy. When electrons hit any intermediate-temperature region in the cavity string, bursts of adsorbed hydrogen gas can sometimes occur and trigger vacuum interlocks. These can lead to redistribution of the adsorbed gas in the cavity string and to lower operating gradients. Therefore, operation into high electron loading must be prevented for the sake of stability, which limits the final linac output beam energy. Each cavity is set at a stable gradient based on the collective limiting gradients achieved through a series of SRF cavity/cryomodule performance tests at SNS, whereas the design calls for setting uniform gradients [3].

At SNS, in situ processing in the tunnel has been identified as an important area of research for improving the SRF cavity performance while minimizing the machine operational impact and reducing the cost of the improvements. Active R\&D for in situ processing using plasma is ongoing at SNS; the results show the potential to increase the accelerating gradients in operating cavities by $2 \mathrm{MV} / \mathrm{m}$ on average. The accelerating gradients of the original cavities for the PPU baseline assume some gradients for both medium- and high-beta cavities have been increased by plasma processing. The initial application of in situ plasma processing to eight installed cavities has exceeded expectations, increasing gradients by an average of $2.5 \mathrm{MV} / \mathrm{m}[4]$.

The high-power RF systems for the original cavities will be kept the same for economic reasons. Since the output power of the original high-power RF systems at saturation is $550 \mathrm{~kW}$, the accelerating gradients of high-performing original high-beta cavities will need to be lowered for the PPU beam loading.

In addition, a spare medium-beta cryomodule will be available for conducting repairs on the inoperable medium-beta cavities, 5a and 11b. It is assumed that one of these cavities will be repaired for operation in advance of the PPU.

If performance improvements after the repairs or in situ plasma processing do not meet expectations, the linac output energy will still be $1.3 \mathrm{GeV}$, but with a reduced energy margin.

Dynamic detuning compensation. The observed dynamic detuning of the original SNS high-beta cavities due to the Lorentz force is in the range of $1-2 \mathrm{~Hz} /(\mathrm{MV} / \mathrm{m})^{2}$ during the whole pulse. The dynamic detuning during the beam is less than $0.5 \mathrm{~Hz} /(\mathrm{MV} / \mathrm{m})^{2}$. Since the cavity bandwidth is relatively large (half bandwidth is $\sim 570 \mathrm{~Hz})$ or the external Q is relatively low $\left(<10^{6}\right)$ because of the high beam loading, the additional RF power needed to compensate for the dynamic detuning is minor. This amount of detuning is well managed by the adaptive feed-forward (AFF) implemented in the SNS low-level RF system. Fast piezo tuners were installed to mitigate any unexpected mechanical resonance conditions. To date, there has been no strong need to use them, so they have never been operated. Moreover, the present SNS piezo tuner design has proved to be mechanically unreliable and will not be part of the tuners for the PPU.

\subsubsection{End Group}

End group thermal stability. The RRR of niobium for the end group of the original cavities is about 70 after heat treatment. The end group is cooled by indirect conduction to the helium circuit. The thermal 
processes of the end group are relatively slow, since the surface magnetic fields are relatively small and the cooling relies on conduction. In terms of thermal stability, the end group can allow fairly large material defects because the field is low and the total thermal load is small. But the end group is sensitive to heating from a broad range of thermal loads - such as thermal radiation from the inner conductor of the fundamental power coupler (FPC), field emission, and multipacting - because of the long conduction path to the thermal sink. As mentioned earlier, most of the original cavities show large field emission, which is the major operational limiting condition, leading to end group heating, gas bursts, and cavity quench [5].

If the RRR of niobium is larger than 150 , the end group will be stable at larger thermal loads against electron loading and/or local heating at the end group. Figure 3.1 shows temperature profiles of the highbeta cavity end group. Thermal radiation from the FPC is assumed to be $3 \mathrm{~W}$ uniformly distributed over the end group surface for a conservative analysis, and the surface magnetic field at the design gradient is used for the $4 \mathrm{~K}$ helium case. For the $2 \mathrm{~K}$ helium case, the end group temperature profile is about the same, except in very close proximity to the helium vessel. Without any other thermal load except thermal radiation from the FPC, the maximum temperature in the end group will be lower by $1 \mathrm{~K}$ for the $\mathrm{RRR}=150$ case. The analysis also indicates that the end group for the $\mathrm{RRR}=70$ case will not be stable with an additional localized external thermal load of 0.2 to $2 \mathrm{~W}$, depending on the location of the external thermal load, whereas it will still be stable with five times larger additional thermal loads for the $\mathrm{RRR}=150$ case.

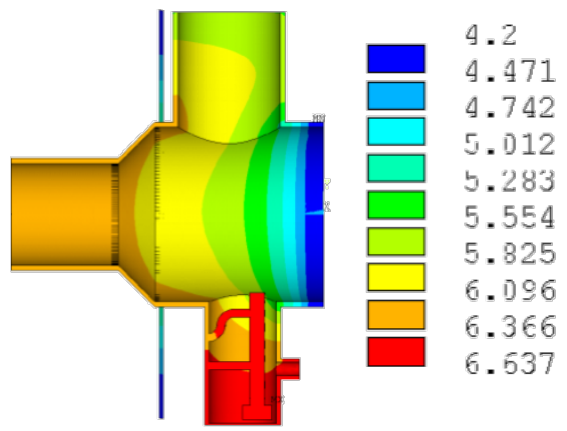

(a) $\mathrm{RRR}=70$

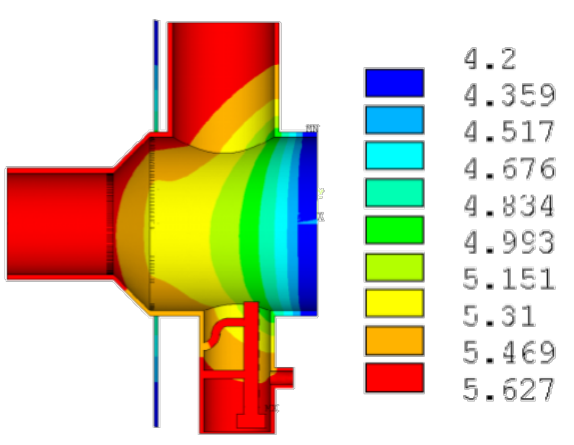

(b) $R R R=150$

Figure 3.1. End group temperature profiles with (a) $R R R=70$ and (b) $R R R=150$.

The other thermally weak location in the original cryomodules is the cavity end group at the field probe side at the warm-to-cold transitions (Figure 3.2). At normal operating conditions, the temperature at this location is about $7-8 \mathrm{~K}$. When there is electron loading, other external thermal loads such as beam halo, or a combination of these around this warm-to-cold transition, the local temperature easily goes to the hydrogen evaporation temperature. This can result in a large vacuum burst, in which the electrons interact with the RF field. Sometimes, a vacuum burst changes the cavity condition drastically, and cavities then require serious conditioning or reduction of the gradient. In the high-beta spare cryomodule, a thermal cooling block cooled by $5 \mathrm{~K}$ supercritical helium was installed at each side.

With these two changes - a higher RRR for the cavity end groups and the cooling blocks for the warm-tocold transition - the whole system will have a much larger thermal margin against thermal radiation from the FPC; thermal loads from electron loading, such as field emission and multipacting; and any other additional external thermal loads. These are all included in the PPU design. 


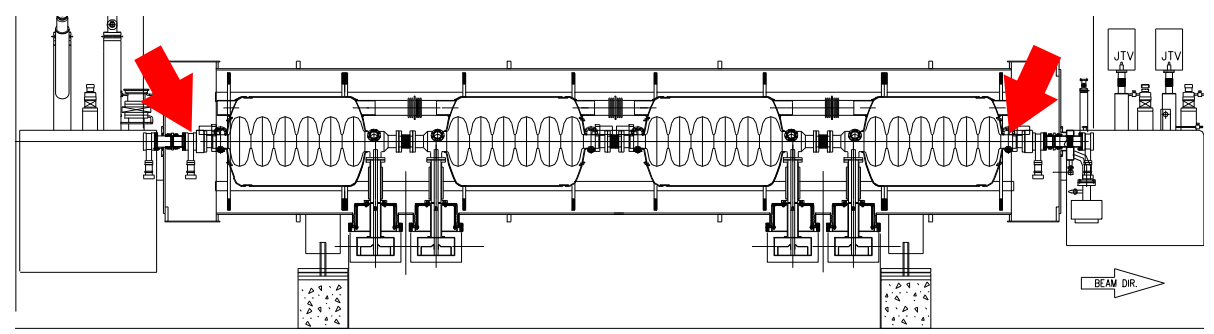

Figure 3.2. Thermal block locations for the $5 \mathrm{~K}$ boundary.

Higher-order mode (HOM) coupler. When an HOM is near the beam spectral lines, the induced voltage could be harmful to the beam and/or generate large HOM-induced power on the RF surface. To address these two main concerns, systematic studies were carried out. In the case of SNS, bunch tracking simulations for both the transverse and longitudinal directions show that beam instabilities are not a main concern if the external quality factor $\mathrm{Q}_{\mathrm{ex}}$ for each $\mathrm{HOM}$ is less than $10^{8}$, the loaded cavity $\mathrm{Q}$ for each nonpi mode fundamental mode has the expected value, and the expected cavity-to-cavity frequency variation is present. With respect to the HOM-induced power issue, general analytic expressions of beam-induced voltages and the corresponding HOM power from the multiple beam time-structures were analyzed and were fully understood by exploring the parameter space of the HOM properties. The damping requirement of each mode is set up in terms of $\mathrm{Q}_{\mathrm{ex}}$ by taking into account the actual HOM frequency behavior of elliptical superconducting cavities. The probability that a cavity HOM frequency will coincide with a beam driving spectral line was estimated to be very small during the linac design phase. However, many factors can cause HOM frequencies to move around over time; and to permanently satisfy the damping requirement, an HOM coupler was installed on each end group of the SNS cavity as a simple precautionary measure $[6,7,8]$.

Unfortunately, operational experience showed that the accelerating mode can sometimes be coupled out and lead to failure of the HOM RF feedthroughs. About 15\% of installed cavities in the SNS SCL are showing abnormal signals through their HOM feedthroughs. All attenuators for the HOM signals were damaged in 1 year of operation during the early days of commissioning and operation. Observations and physical conditions near the HOM couplers imply that HOM coupler failures or degradation are a result of electron activity originating from multipacting, field emission, and even gas discharge at the fundamental mode. A few cavities were inoperable as a result of large coupling with the fundamental accelerating mode. In 2007, the SNS reevaluated the HOM characteristics, including dangerous HOM measurements for all installed cavities. It was concluded that HOM couplers are not needed for the SNS, and it was decided to remove HOM couplers whenever cryomodules are taken out of the tunnel for repairs. Four cryomodules have been taken out of the tunnel so far, and leaks were detected in half of their HOM feedthroughs. Therefore, the PPU cavities will not have HOM couplers.

Refurbishment of the spare cavities and new cavities. Consideration was given to using the existing 20 high-beta spare cavities, produced during the SNS project, in the PPU. However, given the end group design of the original cavities, meeting the gradient specification $(16 \mathrm{MV} / \mathrm{m})$ for the PPU project is a large risk. Currently, a study is in progress in which the end groups were replaced on two spare cavities. For these test cavities, the new end groups were constructed of high-RRR niobium, the HOM cans were removed from the design, and high-aspect-ratio deep drawing was not used for manufacturing of parts. These cavities were delivered from the vendor, and chemical polishing has been completed. Continued testing of the cavities is scheduled for late in calendar year 2016. Figure 3.3 shows a model of the original cavity versus the modified cavity for the PPU. Figure 3.4 depicts the completed end group replacement. 


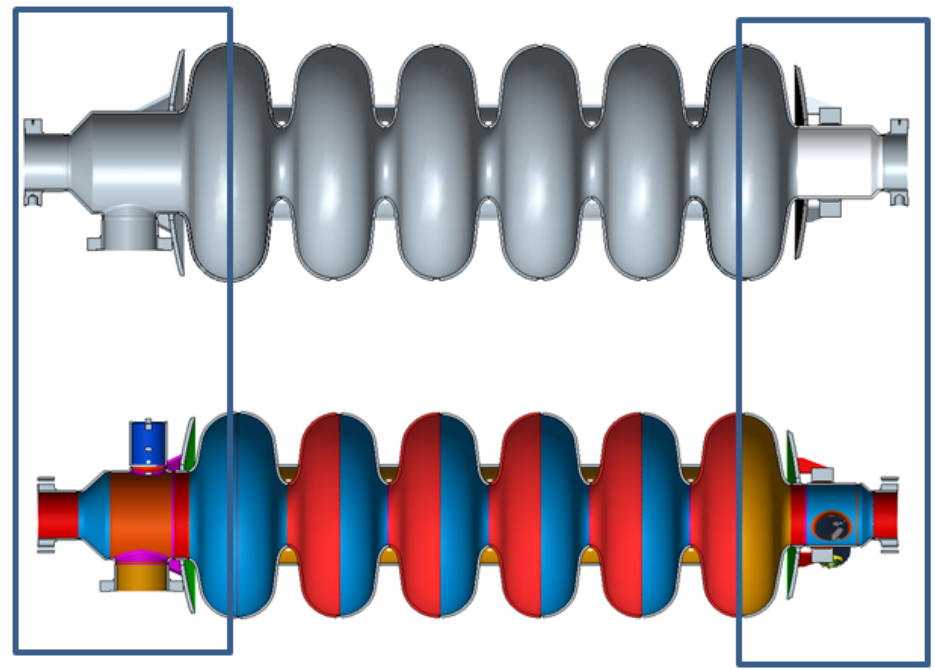

Figure 3.3. End group modification. (Top: cavity with modified end group for the PPU. Bottom: Original SNS high-beta cavity).

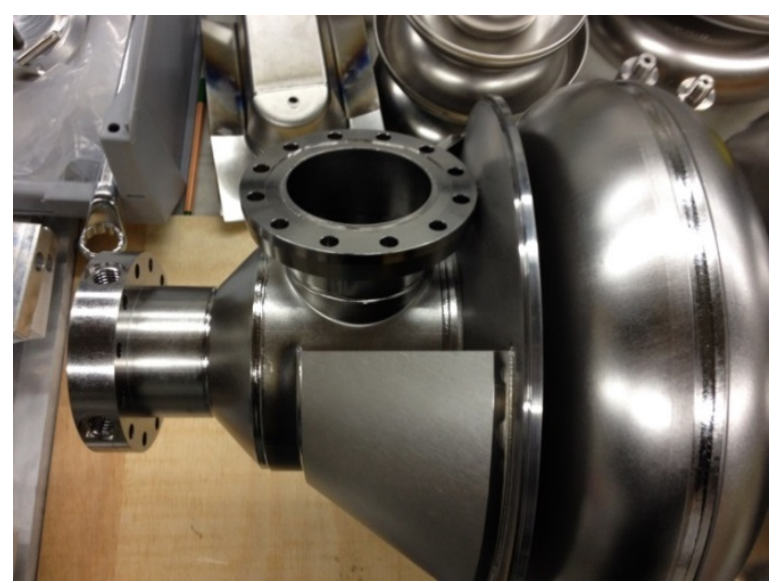

Figure 3.4. Completed end group replacement.

Helium vessel. The helium vessel is constructed of titanium and a niobium/titanium alloy. In the original design, the stiffeners in the head of the vessel were welded to a ring that allowed flexibility in adjusting the final position of the vessel body to the heads of the vessel. Because of the position of this ring, a fullpenetration weld was not possible. The new design eliminated this ring and redesigned the stiffener to fit completely with the heads of the vessel. Therefore, the main body of the helium vessel was lengthened to account for the added length of the slip fit rings. The main body of the vessel will be attached to the heads of the vessel via full-penetration welds. This arrangement more closely meets the intent of the ASME Boiler and Pressure Vessel (B\&PV) Code. Figure 3.5 shows the original helium vessel design versus the new design. This modification was successfully incorporated in the spare high-beta cryomodule. 

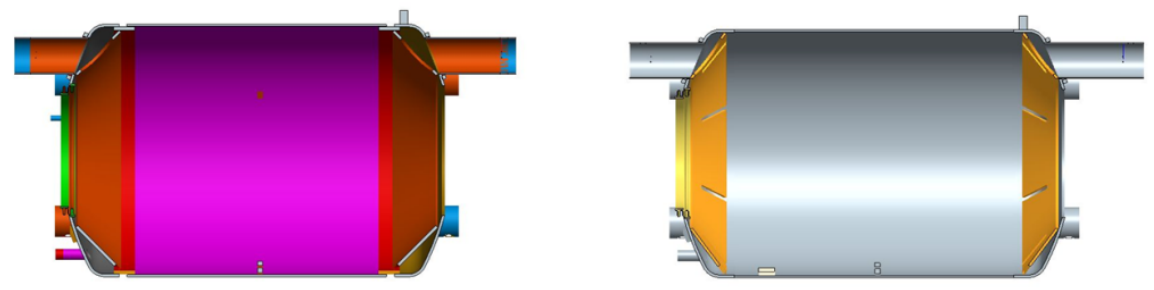

Figure 3.5. Original (left) versus new (right) helium vessel designs.

\subsubsection{Fundamental Power Coupler}

The original SNS FPC is designed and scaled from the one KEK developed for 508.8 MHz. A simple window geometry was chosen to facilitate manufacturing and assembly. The design of the SNS FPC relies on a simple coaxial line at $50 \Omega$. The planar alumina window includes impedance-matching elements, as well as a TiN anti-multipacting coating. The chokes at both sides of the ceramic window have the important role of improving the window matching. The depths of the chokes were optimized in the simulations to allow sufficiently low return loss and insertion loss [9]. The transition between the WR975 waveguide from the klystron and the coaxial line of the FPC is provided by a doorknob configuration. Even a small mechanical change inside the structure can result in a significant change in the RF performance; such disturbances include grooves, slits, rounded corners, and others. The FPC includes the possibility of biasing the inner conductor, via a capacitor gap between the doorknob and the inner conductor itself, at variable voltages between -2.5 and $+2.5 \mathrm{kV}$. The gap is filled with Kapton foil, which is capable of withstanding the biasing voltage. The requirement for the maximum allowable thermal radiation from the FPC to the end group is $2 \mathrm{~W}$. The outer conductor of the FPC has a direct conduction path to room temperature. To minimize heat transfer to the end group, the FPC outer conductor is designed to be cooled by supercritical helium at $3 \mathrm{~atm}$ and $5 \mathrm{~K}$ with a $0.04 \mathrm{~g} / \mathrm{s}$ mass flow rate, which removes about $30 \mathrm{~W}$ of static and dynamic heat load. The inner surface of the outer conductor is designed to be copper-plated with $15 \mu \mathrm{m}(\mathrm{RRR}=10)$ of copper on stainless steel to reduce wall dissipation. The inner conductor of the FPC is made of copper and does not have active cooling. Figure 3.6 shows a FPC schematic, the inner conductor assembly, and the outer conductor assembly.
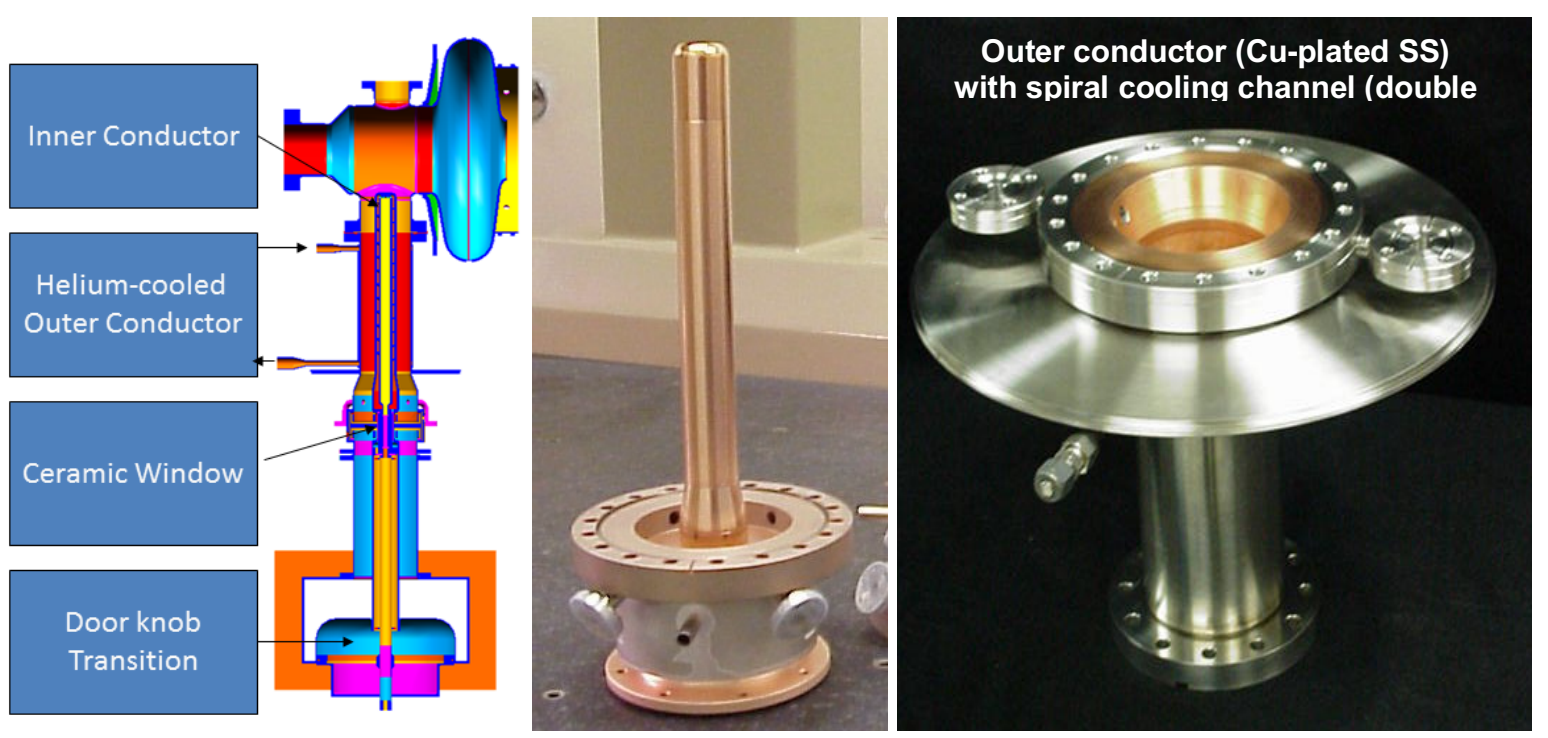

Figure 3.6. SNS fundamental power coupler (left: schematics, middle: inner conductor assembly, right: outer conductor assembly). 
The original FPCs were tested at up to a $2 \mathrm{MW}$ peak, a $0.6 \mathrm{~ms}$ long RF pulse width, and 60 pulses per second (pps) for the full traveling wave condition and in excess of $600 \mathrm{~kW}$ at a $1 \mathrm{~ms}$ long RF pulse width and $60 \mathrm{pps}$ for full standing wave mode in the test stand. The FPCs have been reliably performing at over $550 \mathrm{~kW}$ peak power in real cavity operation at various standing wave ratios limited by the operational envelope in the linac. The FPC for the PPU cavities must be able to transfer up to $700 \mathrm{~kW}$ peak power over a $1.3 \mathrm{~ms}$ pulse width at a repetition rate of $60 \mathrm{~Hz}$. Based on the testing and operational experience, the RF performance of the original FPC can satisfy the PPU requirements; however, a design modification is needed to maintain the inner conductor temperature at a sufficiently low level. Since the thermal radiation from the inner conductor will be higher as a result of the increased average RF power, a thicker inner conductor will be used for the PPU, which is sufficient and provides the simplest solution. Active cooling for the inner conductor was also taken into account, if the passive enhancement of cooling with the thicker inner conductor is not adequate. Active cooling will require a more complex design configuration and may result in operational difficulties at an upset condition. The temperatures are calculated for various inner conductor thicknesses at both full traveling and standing wave conditions. During pulsed operation, the actual condition in the FPC is between full standing and full traveling wave conditions, closer to the full traveling wave condition. Figure 3.7 shows comparisons of inner conductor tip temperatures. With a $7 \mathrm{~mm}$ thick inner conductor, the inner conductor tip temperature at the PPU condition can be kept below that of the FPCs presently operating at SNS, which have an inner conductor thickness of $3 \mathrm{~mm}$. The prototype FPC for the PPU with the increased wall thickness was manufactured by the vendor that provided the original FPCs, installed in a horizontal test apparatus, and successfully tested. Except for the wall thickness of the inner conductor, all other designs are exactly the same. The $\mathrm{Q}_{\mathrm{ex}}$ value chosen for the PPU in Table 3.1 is optimized to reduce the RF power requirement.

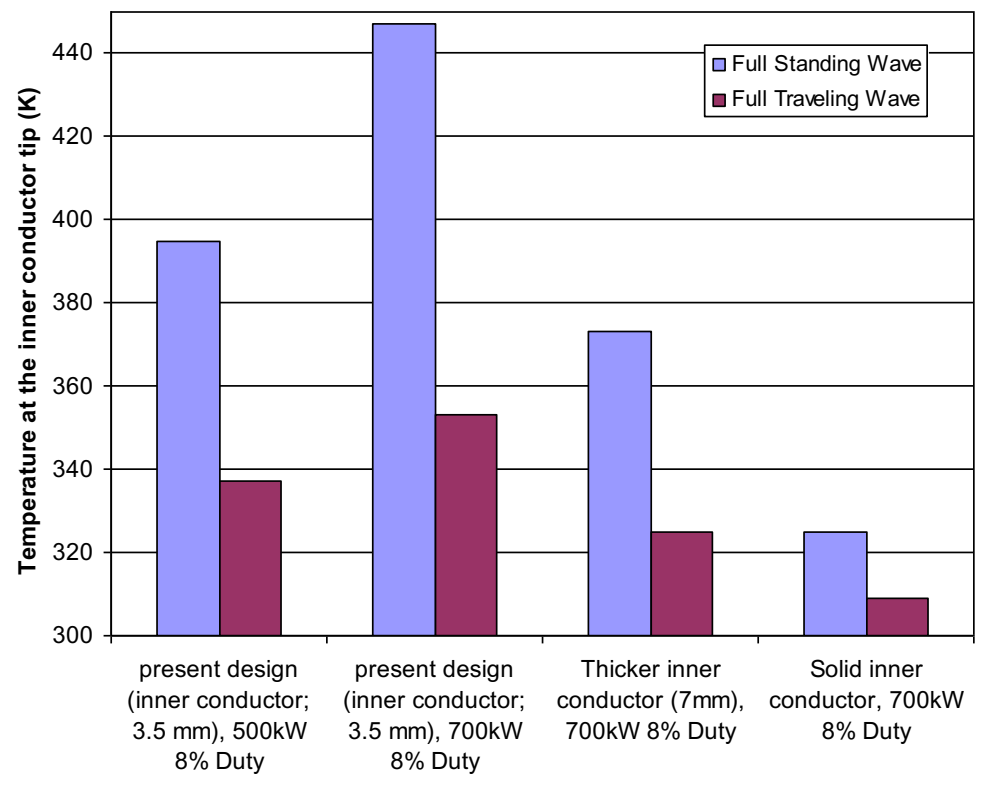

Figure 3.7. Comparisons of calculated inner conductor tip temperatures.

\subsection{CRYOMODULES}

The SNS SCL consists of 23 cryomodules, 11 of which are medium-beta and 12 high-beta. The mediumbeta cryomodules each contain three six-cell elliptical cavities, whereas the high-beta cryomodules contain four six-cell elliptical cavities. The cavities are described in Sect. 3.1. The design was based on the Continuous Electron Beam Accelerator Facility cryomodule, with improvements inspired by the 
Large Hadron Collider, TESLA (Tera-electron-volt Energy Superconducting Linear Accelerator), and the Jefferson Laboratory $12 \mathrm{GeV}$ upgrade design efforts [10]. Figure 3.8 shows the elevation view of the original high-beta cryomodule design by Jefferson Laboratory.

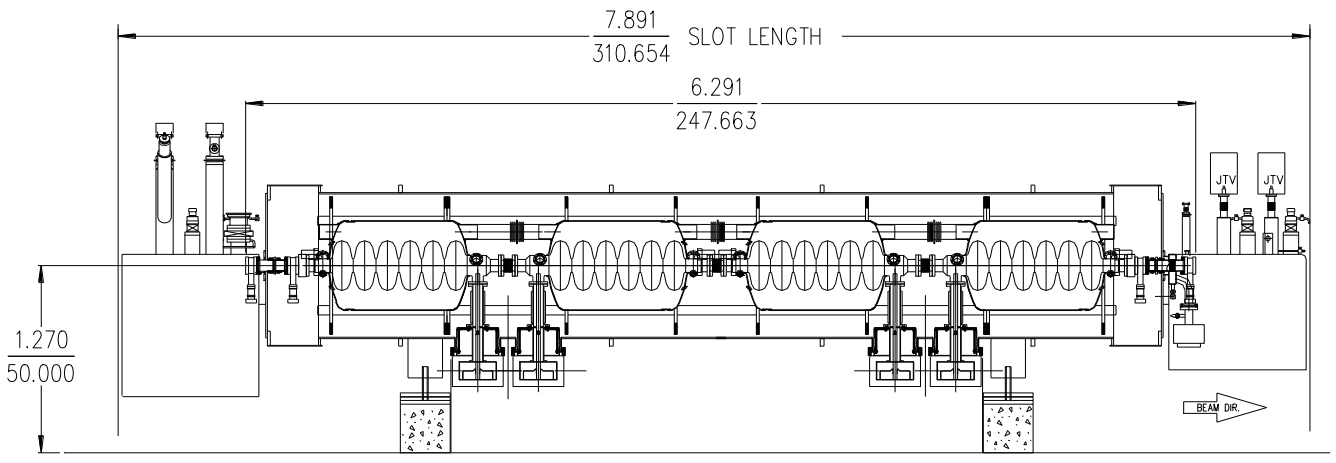

Figure 3.8. The original high-beta cryomodule.

The cryomodule consists of a cavity string, a space frame, a thermal shield, two layers of magnetic shielding, a vacuum vessel, and two end cans. The general arrangement of these components is depicted in Figure 3.9.

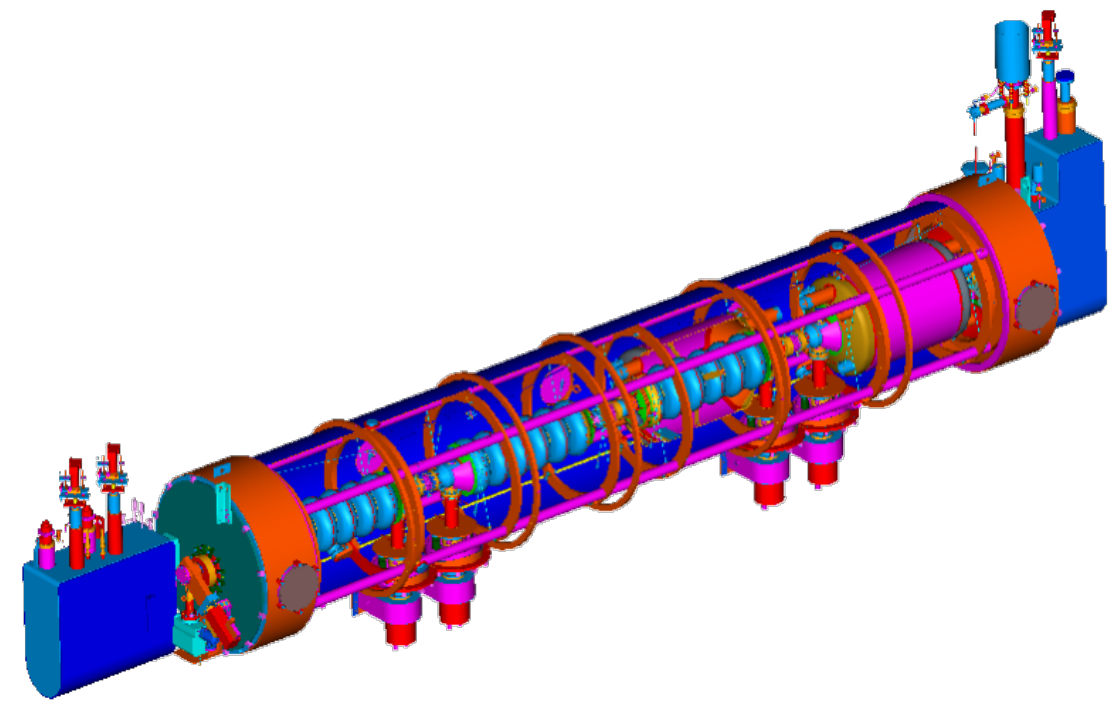

Figure 3.9. Cut view of the original high-beta cryomodule.

During the PPU project, seven cryomodules similar to the original high-beta cryomodules will be installed in the linac tunnel. The relevant parameters for the mechanical design of the original high-beta cryomodules, which remain as design constraints for the new cryomodules, are listed in Table 3.3.

Table 3.3. Design constraints for the PPU cryomodules

\begin{tabular}{lc}
\hline \multicolumn{1}{c}{ Parameter } & Value \\
\hline Slot length & $7.891 \mathrm{~m}$ \\
CM length (bore tube) & $6.291 \mathrm{~m}$ \\
Number of bayonets & 4 \\
Number of control valves & 5 \\
\hline
\end{tabular}


In 2012, SNS completed fabrication and testing of a spare high-beta cryomodule. The approach to the engineering design for this cryomodule was to maintain critical features of the original design, such as bayonet positions, coupler positions, cold mass assembly, and overall footprint. The components that were fixed in location and those that were considered movable are shown in Figure 3.10. This new cryomodule design was required to meet the pressure requirements put forth in 10 CFR 851, "Worker Safety and Health Program." The most significant engineering change was applying Section VIII of the ASME B\&PV Code to the vacuum vessel of this cryomodule, instead of using the traditional designs in which the helium circuit is the pressure boundary. Applying the B\&PV Code to the helium circuit within the cryomodule was also considered. However, it was determined to be schedule-prohibitive because it required a code case for the niobium materials, which are not currently covered by the code. Good engineering practice, however, was applied to the internal components to verify the quality and integrity of the entire cryomodule [1]. Details of the changes to the vacuum vessel and end cans to apply the code to these components are detailed in Sects. 3.2.3 and 3.2.4, respectively.

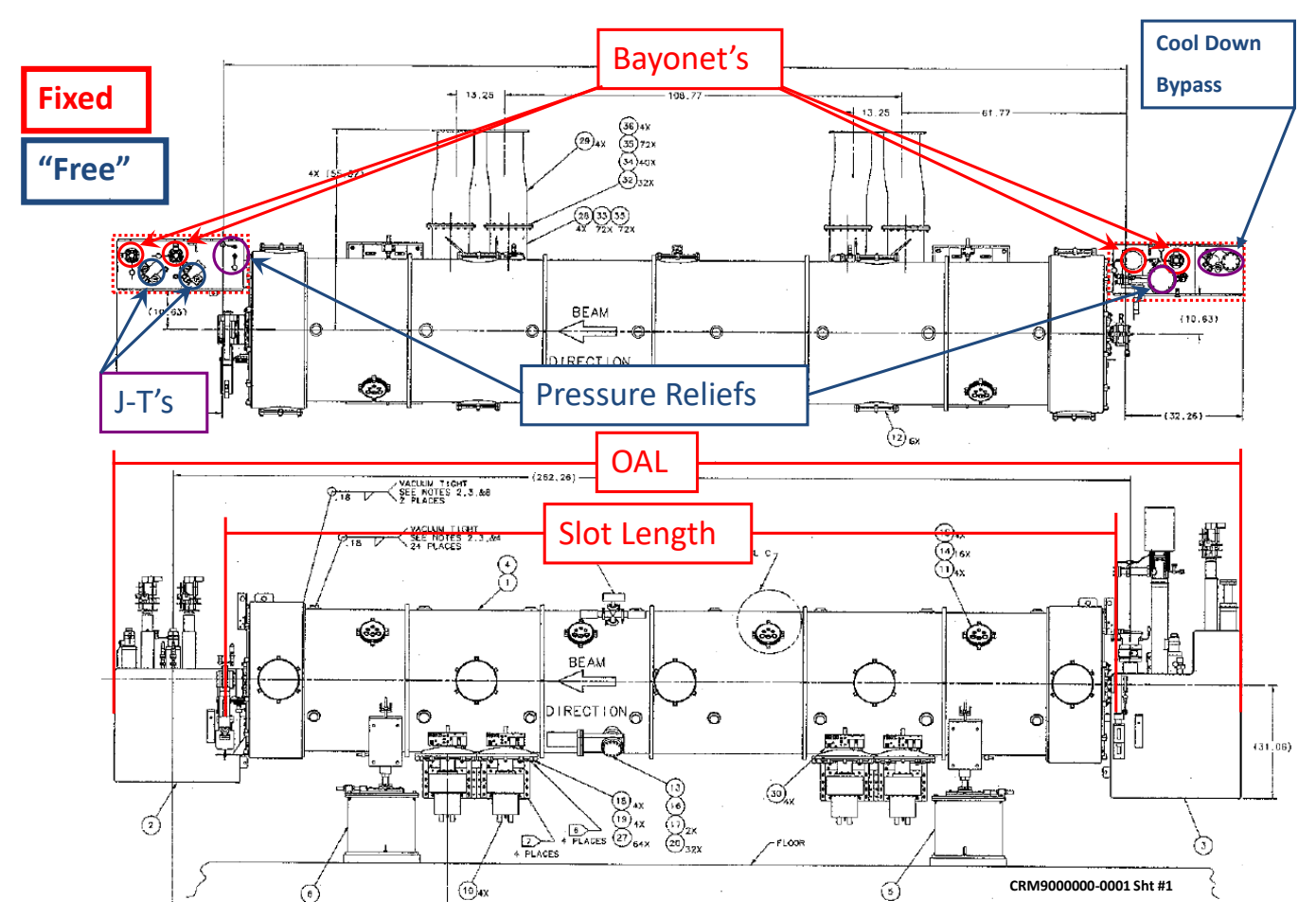

Figure 3.10. Cryomodule reference interface locations.

Several other improvements were made to the spare high-beta cryomodule beyond the changes implemented as a result of applying the B\&PV Code. The HOM feedthroughs on the end groups of the cavities were blanked. The helium vessels were upgraded to meet the intent of the code. Cooling blocks were added to the outside end groups of the cavity string to thermally stabilize these regions. Cold instrument feedthroughs were minimized in the design to prevent possible leaks. All of these improvements are discussed in detail in this report. The completed high-beta spare cryomodule is shown in Figure 3.11. 


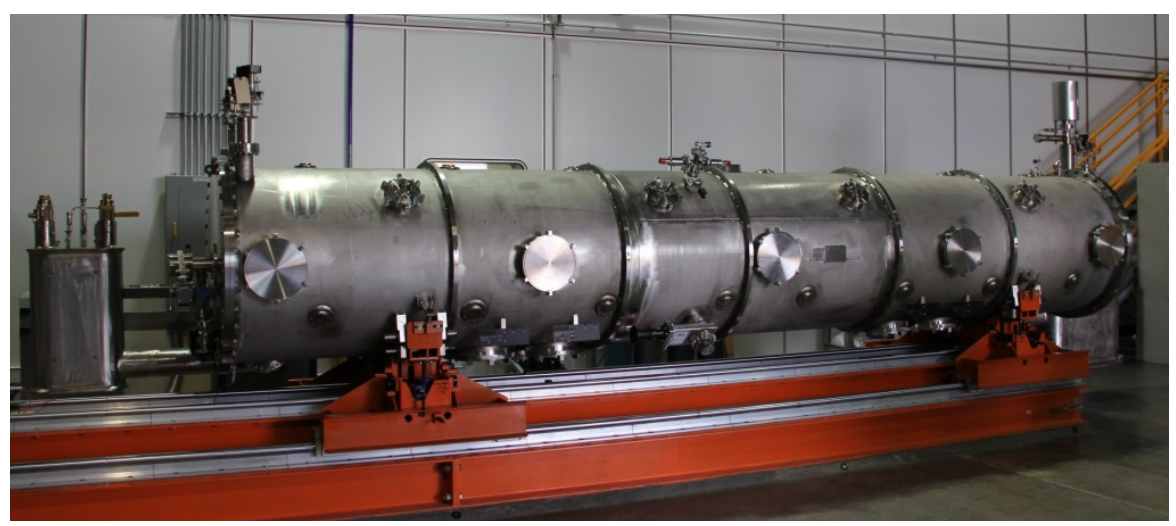

Figure 3.11. SNS spare high-beta cryomodule.

The spare high-beta cryomodule serves as the baseline design for the PPU cryomodules. A couple of modifications to this design will be necessary for the cryomodule to function according to the requirements of the PPU. The cavities will be upgraded as detailed in Sect. 3.1 and an FPC will be used as discussed in Sect. 3.1.3. Other than these two changes, the PPU cryomodule is the same design as the spare high-beta cryomodule. Methodologies for fabrication and assembly were developed and staff expertise was gained during the assembly of the high-beta spare.

\subsubsection{Head Loads and Helium Circuits}

There are three cooling circuits within the cryomodule. Primary cooling provides the liquid helium that cools the cavities within the helium vessels. Secondary cooling provides supercritical helium cooling to the couplers. Because the FPC requires $5 \mathrm{~K}$ supercritical helium to cool the outer conductor, as mentioned in Sect. 3.1.3, the Large Hardon Collider concept of producing $2 \mathrm{~K}$ helium in the cryomodule rather than in the refrigerator is used [10]. This drives the design of placing individual heat exchangers in each of the cryomodules rather than locating one large heat exchange in the refrigerator. In addition, efficiencies are improved with this heat exchanger placement. Shield cooling provides cooling to the thermal shield located in the cryomodule and transfer lines. Figure 3.12 is the updated flow diagram for the spare highbeta SNS cryomodule. The PPU cryomodules will have the same helium circuits as the spare high-beta cryomodule.

The primary helium flow to the cryomodule will be split into two circuits, the primary and the secondary. The primary helium flow will enter the cryomodule in the supply end can and flow through the heat exchanger in the return end can, through the primary Joule-Thomson (JT) valve located on the supply side of the cryomodule, and into the helium vessels. Helium will exit the helium vessels and flow either out the cooldown circuit or through the heat exchanger back into the return transfer line. The cooldown path will be used to imbalance the heat exchanger during the cooldown of the cryomodule so that a desired cooldown rate of $100-150 \mathrm{~K} / \mathrm{h}$ can be achieved.

The secondary circuit will flow through a secondary JT valve, a cooling block on the end group of the supply side cavity, each of the FPC flanges, a cooling block on the end group on the return side cavity, into a surge tank, and out to four parallel paths to the FPC outer conductors. The return from the couplers will flow though metering valves for each of the couplers. The four flows will be combined and discharged into the cooldown header and act as a liquefaction load on the refrigerator. 


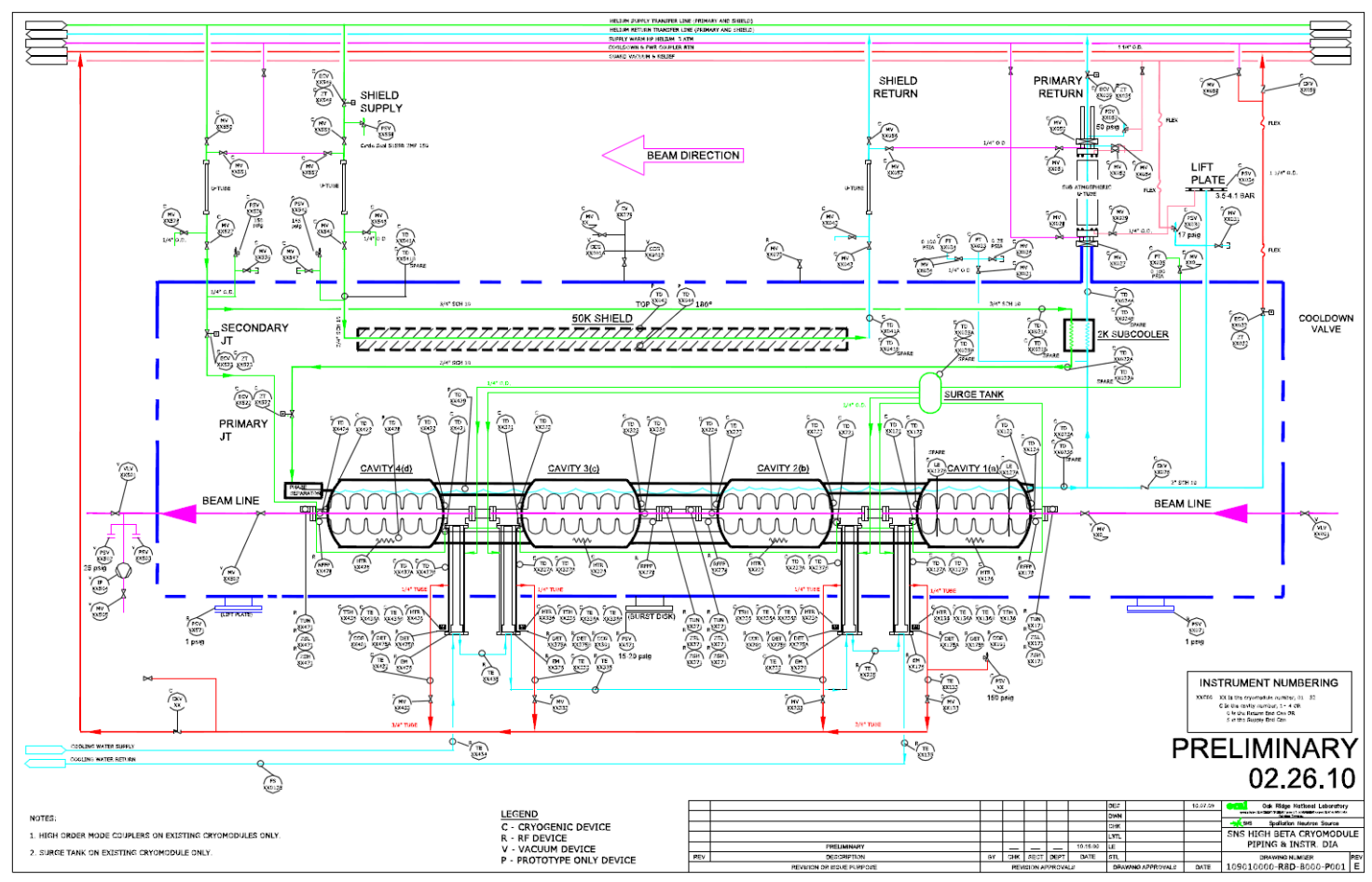

Figure 3.12. Helium flow schematic and instruments for the spare high-beta cryomodule.

The shield flow will be supplied from the turbine 1 outlet in the main cold box at approximately $38 \mathrm{~K}$. It will flow from the turbine outlet, through the transfer line, and into the cryomodules through the shield supply valves. Helium will then flow through the cryomodule and back into the return transfer line, where it will return to the medium-pressure header in the main cold box. The design criteria for the original high-beta cryomodule [11] are listed in Table 3.4, along with those for the PPU based on the operating experience of the spare high-beta cryomodule.

The original SNS cryogenic system was designed with a 100\% capacity margin from the primary and secondary circuits and a $35 \%$ margin for the shield passage. This was done to account for the uncertainty of the design, to mitigate risk to the project, and to ensure capacity for upgrade. With these margins in place, it is anticipated that the addition of seven cryomodules to the linac tunnel will be well within the capability of the SNS cryogenic system. See Section 3.3 for more detailed cryogenic system information.

Table 3.4. Cryogenic load design values for the high-beta cryomodule

\begin{tabular}{lcc}
\hline \multicolumn{1}{c}{ Parameter } & Original cryomodule & PPU cryomodule \\
\hline 2 K heat load (static/dynamic) & $28 / 20 \mathrm{~W}$ & $25 / 40 \mathrm{~W}$ \\
Coupler flow & $0.075 \mathrm{~g} / \mathrm{s}$ & $0.067 \mathrm{~g} / \mathrm{s}$ \\
Shield load including transfer line & $200 \mathrm{~W}$ & $200 \mathrm{~W}$ \\
\hline
\end{tabular}

\subsubsection{Cold Mass}

The cold mass of the cryomodule consists of the cavity string, space frame, $50 \mathrm{~K}$ thermal shield, magnetic shield, and insulation. The cold mass of the spare high-beta cryomodule just before the second layer of magnetic shielding was installed is shown in Figure 3.13. 


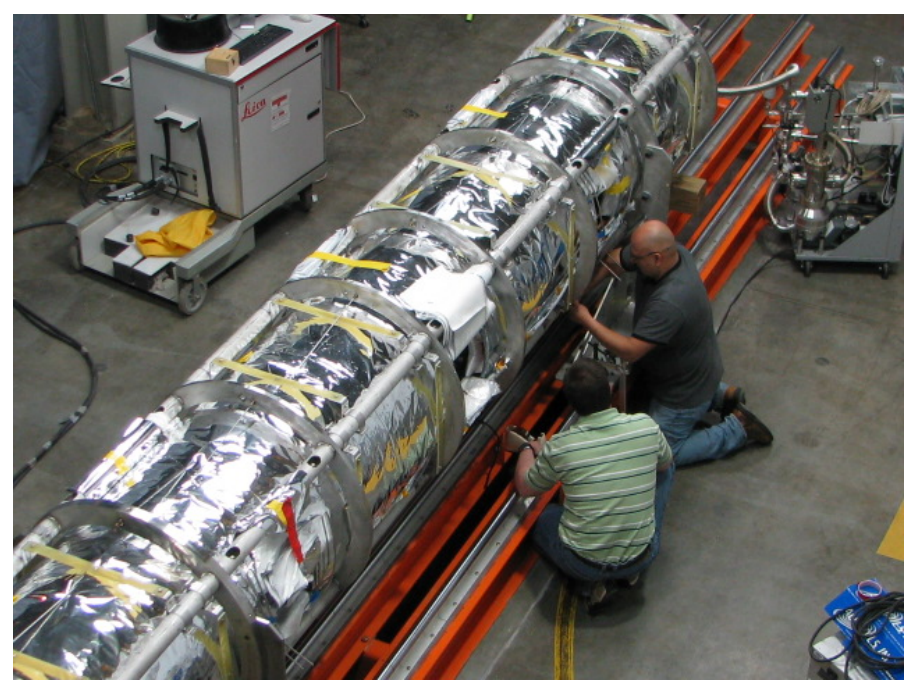

Figure 3.13. Cryomodule cold mass assembly.

The original SNS tuner design shown in Figure 3.14 has been adapted from a Saclay design for the TESLA Test Facility cavities [12]. It is attached to the cavity at three points - two standard standoffs and one piezo tuner. The tuner is adjusted by a motor and harmonic drive unit. This provides a tuning range of approximately $400 \mathrm{kHz}[13]$.

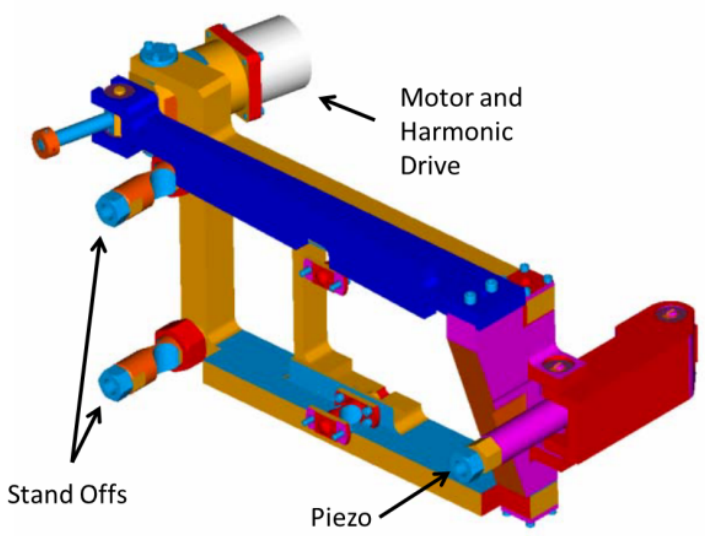

Figure 3.14. Schematic of the original SNS tuner assembly.

One key change to the original cavity string assembly is the removal of the piezo tuner from the tuner assembly. The original design included the piezo tuner to mitigate any unexpected mechanical resonance conditions driven by the Lorentz force. Since the SNS cavity RF circuit has a large bandwidth, and the SNS cavities do not have adverse mechanical resonance conditions, the cavity phase and RF amplitude are well managed within the requirements by the AFF implemented in the SNS low-level RF system [14]. The piezo tuners have never been actuated in operation at SNS. Moreover, several failures with piezo stacks have occurred because of pressure changes in the cryomodules either during the 2 to $4 \mathrm{~K}$ transition or during upset conditions. The piezo tuners were replaced with the standard standoffs used on the other two legs of the tuner. Because of this history, it was decided to eliminate them from future cryomodules built for SNS. 
Thermal and magnetic shielding is incorporated in the design of the cold mass. The cavity string is surrounded by a thermal shield that operates at approximately $50 \mathrm{~K}$. This shield is wrapped in multilayer insulation and provides a thermal radiation barrier between the cavities and the ambient environment. The thermal shield used for the PPU cryomodules is very similar to the original shields used for the original cryomodules. Two layers of magnetic shielding are incorporated into the cold mass assembly. One layer is installed on the outside of the helium vessels within the cavity string, and the other on the outside of the space frame. These layers reduce the Earth's magnetic fields by a factor of 20 or higher to minimize the effect on cavity operation. The only change to the shields is to adjust their geometry to properly fit within the new B\&PV Code-compliant vacuum vessel.

Another component of the cold mass is the space frame, which was developed at Jefferson Laboratory to facilitate the installation of long cavity strings into cryomodules at a relatively low cost. Because the original cryomodules were assembled at Jefferson Laboratory and shipped to SNS, the space frame was strengthened to handle the transportation load while maintaining the alignment of the cavities. This same design will be used in the PPU cryomodules. The space frame provides support to the cavity string through nitronic rods. The space frame is supported from the vacuum vessel. The use of this system to support the cavity string limits the conduction of heat to the cavities. Nitronic rods were selected to support the transportation loads because of their high strength. An alternative of using stainless steel may be considered for the PPU cryomodules.

\subsubsection{Vacuum Vessel and Support Stands}

To meet the new B\&PV Code, the decision was made to define the vacuum vessel and end can envelope as the pressure boundary because of the difficulty in applying the pressure code to materials in the helium circuit. The niobium, titanium, and niobium-titanium alloys are not code-listed at the operating temperature that is routinely maintained within the cryomodules. The approach of using the vacuum vessel as the pressure boundary made use of an interpretation of VIII-1-89-82 in which it was deemed acceptable to stamp the exterior vessel of a heat exchanger if the tube side exceeds the rated operating pressure, provided the shell and associated relief devices are designed to withstand the highest design pressure associated with the tube side. Moving the pressure boundary from the cavity helium circuit to the vacuum vessel has additional safety benefits: (1) The vacuum shell material is 304 stainless steel, which is one of the best materials for fracture toughness and ease of fabrication. (2) The vacuum shell will never reach the helium operating temperature even with a catastrophic failure of the helium lines because of the thermal mass of the vessel material, which is at room temperature. Therefore, the material properties at LN2 temperature can be used. (3) The vacuum vessel envelope could be easily pressure-tested without the SRF cavity string installed [15].

Enacting the code allowed the removal of the bridging ring from the original design concept. That required that the main part of the vacuum vessel in the spare cryomodule be longer than the original cryomodules. Removal of the bridging ring complicated assembly in the warm-to-cold transition region, necessitating design changes to this region of the cryomodule. The changes affected the flexibility of the alignment of the string to the warm beam line flange. Therefore, modeling of the string within the vacuum vessel had to be very precise because the movement of the warm-to-cold transition in the old design was eliminated. The alignment during the spare high-beta cryomodule assembly was performed with a laser tracker, and the modeling was successful, so that the string aligned with the warm valve within the specification limit of $1 \mathrm{~mm}$ [1]. Figures 3.15 and 3.16 depict changes in the design of the vacuum vessel. 

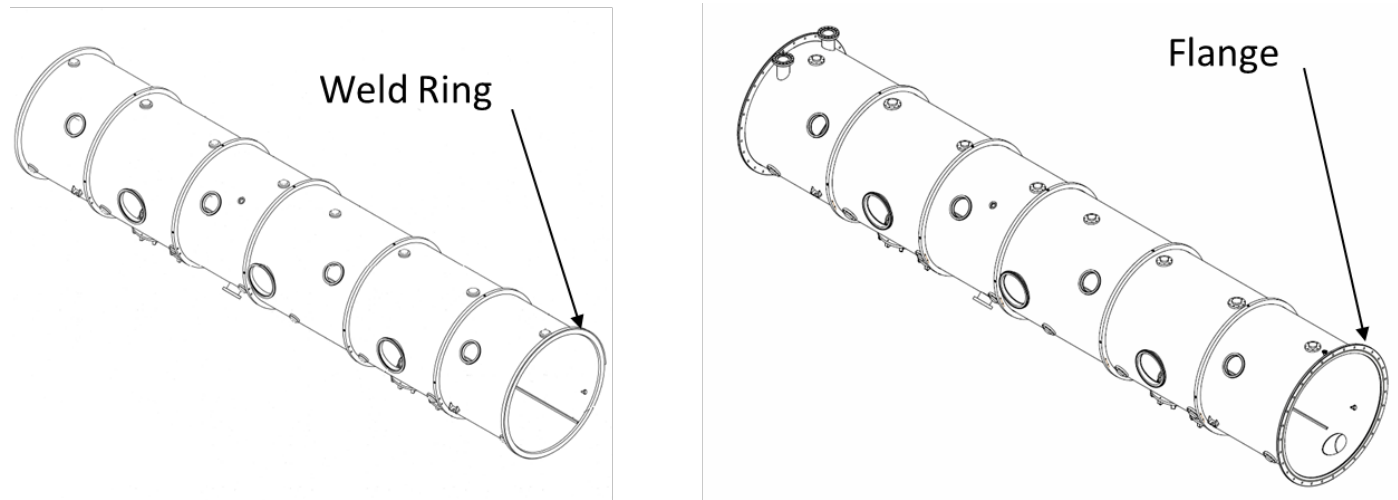

Figure 3.15. Original (left) versus new (right) vacuum vessel.
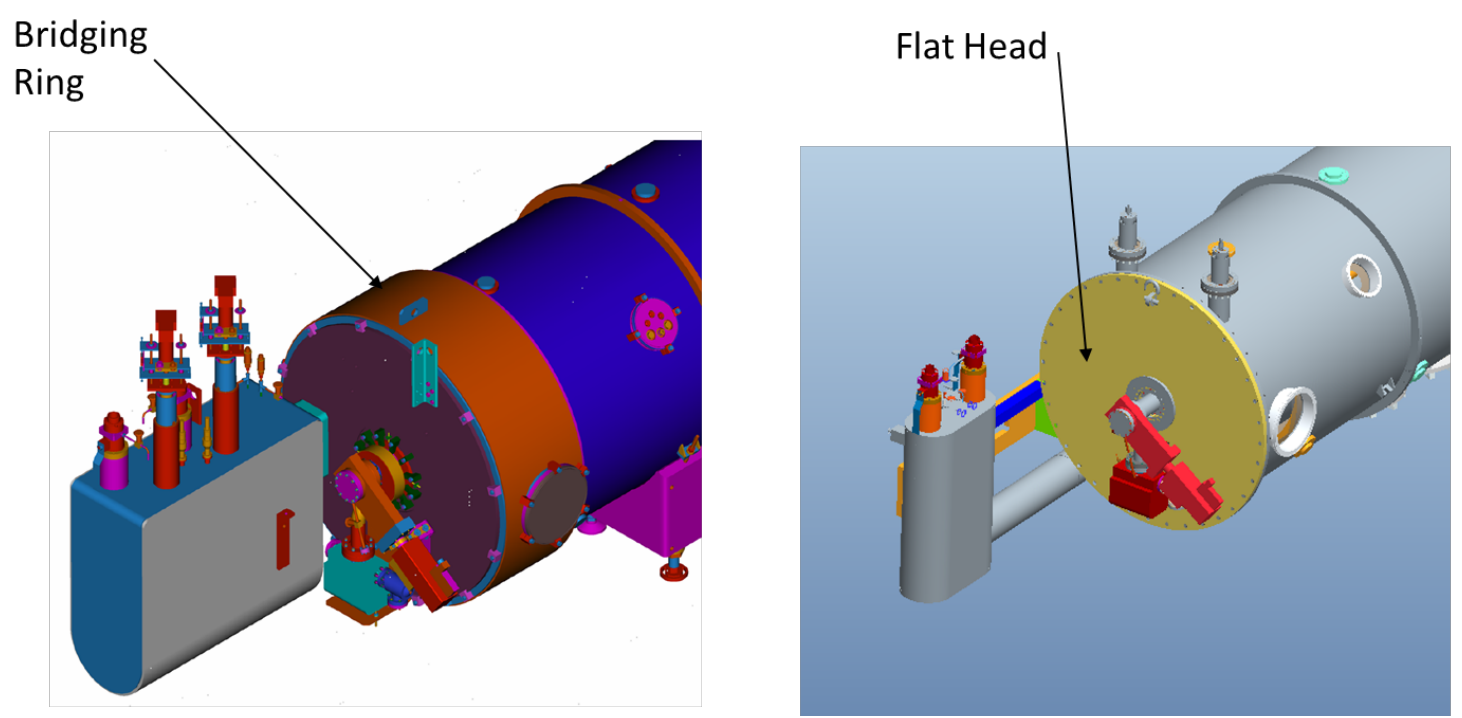

Figure 3.16. Original (left) versus new (right) vacuum jacket design.

To simplify the supply end can as much as possible, the primary and secondary JT valves were moved from the end can to the vacuum vessel. This required a change in the vacuum vessel design, and more piping was added to the main body of the cryomodule. Figure 3.17 depicts this change.
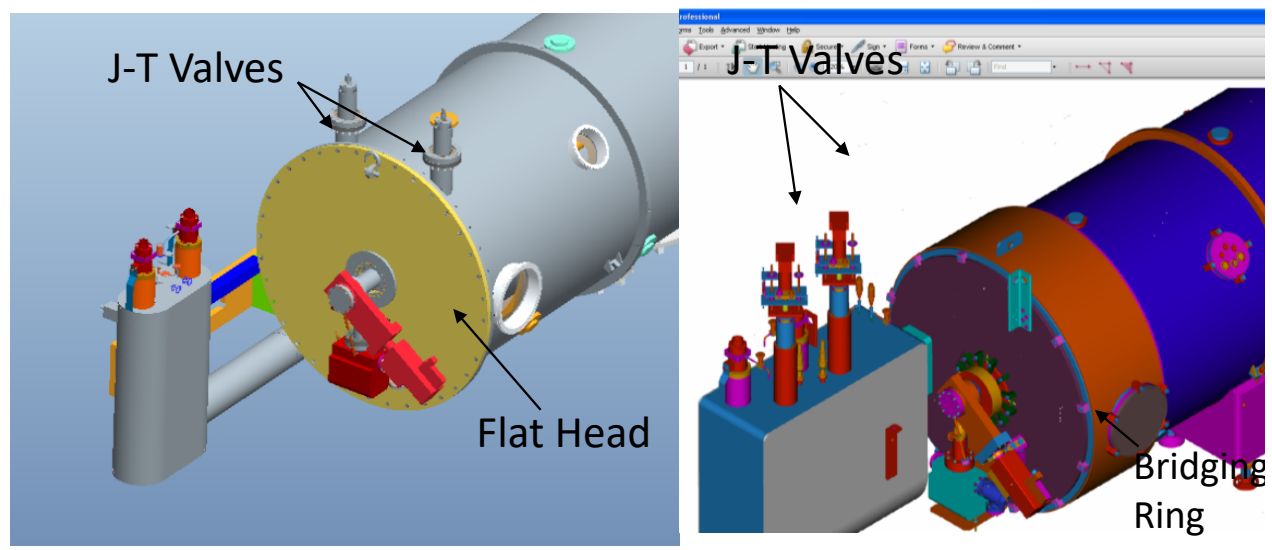

Figure 3.17. JT valve positions for the PPU cryomodule (left) and the original (right) cryomodule. 


\subsubsection{End Cans}

In designing the high-beta spare cryomodule, multiple changes were made to the end cans. Previously, the end cans had not been designed or fabricated in such a way that the vacuum boundary could withstand pressure. Therefore, the shape and the thickness of the end cans were modified. Because of the design approach of having three separate pressure stamps, the end cans had to be capable of being pressuretested individually; the same was true for the vacuum vessel. The end cans attach to the side of the vacuum vessel by a flanged connection, an arrangement that allows easy pressure testing of all three vessels.

The piping within the supply end can was significantly reduced. The new supply end can design is compared with the original design in Figure 3.18. A similar analysis was conducted to simplify the return end can by moving the heat exchanger into the vacuum vessel. To do so, it would have been necessary to change the heat exchanger. However, the decision was made to keep all of the original equipment in the return end can, since that was a proven and reliable design. The piping in the return end can was simplified as much as possible for ease of fabrication (Figure 3.19). Because the new pressure-rated end cans were connected differently to the vacuum vessel, they required new mechanical support brackets, as shown in Figure 3.20.
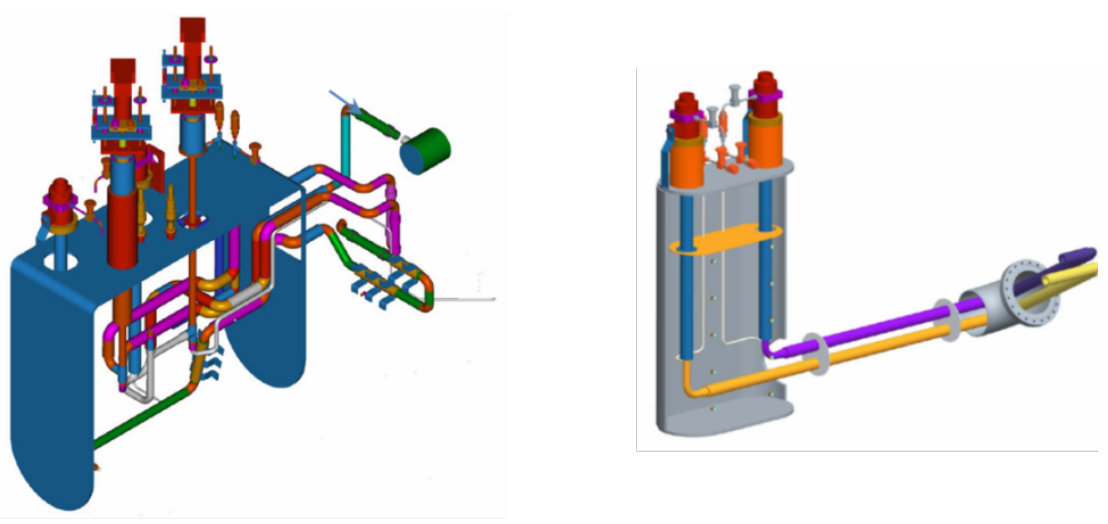

Figure 3.18. Original (left) versus new (right) supply end can.
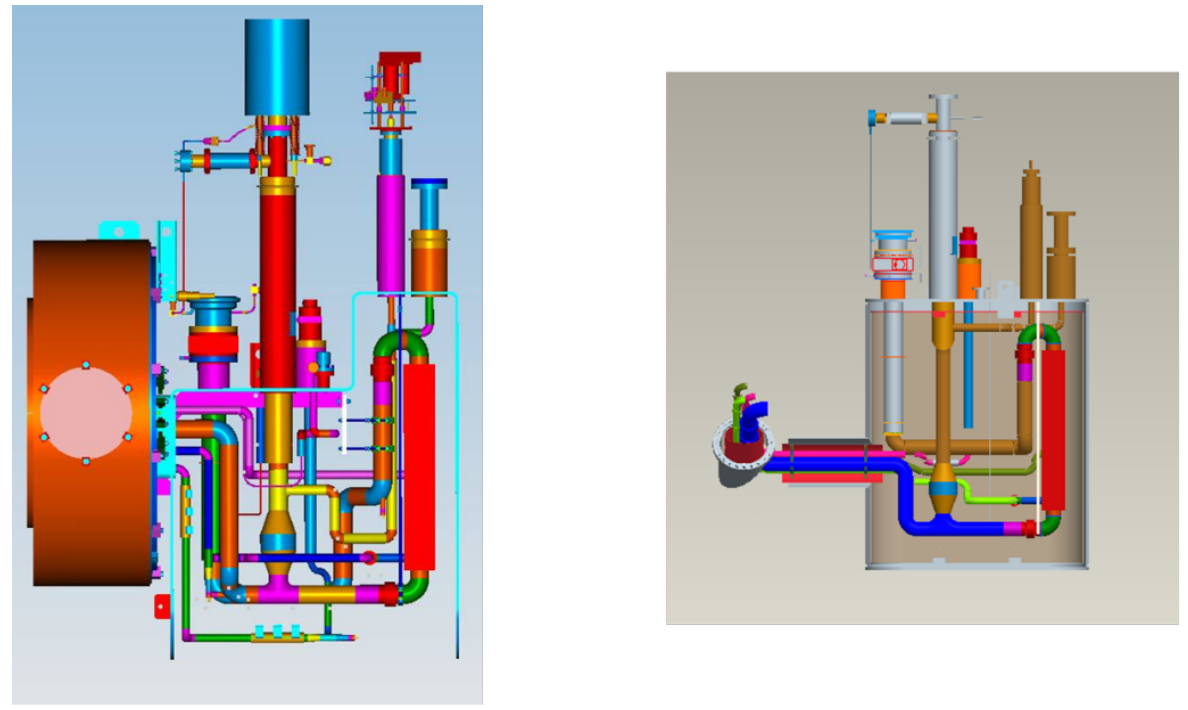

Figure 3.19. Original (left) versus new (right) return end can. 


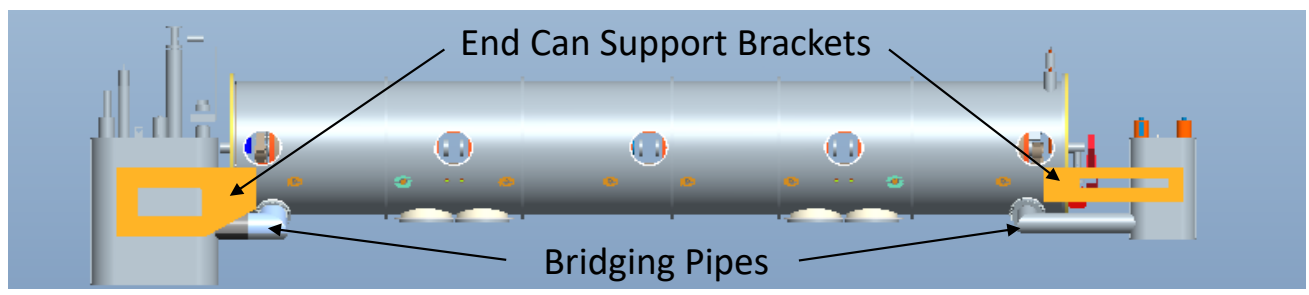

Figure 3.20. Interfaces between end cans and vacuum vessel for the PPU cryomodule.

Although the helium circuit is not code-stamped under this design philosophy, good engineering practice was applied to this portion of the cryomodule for the spare high-beta cryomodule. The helium vessels were modified so that the stiffening in the heads of the vessels was increased. In addition, the design of the cylindrical portion of the helium vessel was modified so that all welds could be full-penetration welds. The fabrication techniques used were consistent with ASME code practices and procedures. The inspection requirements were also consistent with those called out in the ASME B\&PV Code. All welds that would eventually be subjected to cryogenic temperatures were made using low-ferrite filler material. Ferrite is known to reduce toughness in cryogenic applications; therefore, the filler material had a ferrite number below 5 [16].

\subsubsection{Instrumentation Hardware}

The original cryomodules were equipped with many in-process diodes for accurate temperature measurement of helium streams. The in-process diodes required a cold instrument feedthrough to bring the wires out of the process space into the insulating vacuum space. During welding for the original cryomodule fabrication, the ceramic portions of some of the feedthroughs were overheated. Many leaks in the cryomodules were observed before the operation of the SNS linac. Multiple repairs were performed by cutting through the insulating vacuum boundary and thermal shield to access the diodes. In the new design for the spare high-beta cryomodule, the cold feedthroughs were removed and replaced by surfacemounted diodes. These surface-mounted diodes have given reliable temperature feedback, and operation using these readings has been successful. The diodes located on the cavity surfaces at the top and bottom of each cavity were also relocated to the outside of the helium vessel for the spare high-beta cryomodule. Using these diodes to determine the cooldown rate has been proved to be very similar to using the cryomodule with diodes directly mounted to the cavities.

Because of the high radiation levels within the tunnel, the pressure transmitters used on the original SNS cryomodules failed in a short time. They were replaced by Honeywell strain gauges with no internal electronics, making them much more reliable in a high-radiation environment.

\subsection{CRYOGENICS}

The SNS cryogenic system design closely resembles that of the Thomas Jefferson National Accelerator Facility cryogenic system with some modifications [17]. The SNS cryogenic system was designed to provide refrigeration capacities of $125 \mathrm{~g} / \mathrm{s}$ at $2.1 \mathrm{~K}, 8300 \mathrm{~W}$ of shield cooling, and $15 \mathrm{~g} / \mathrm{s}$ of liquefaction flow. This cryogenic capacity is sufficient to accommodate the PPU project, and no additional equipment is required for the central helium liquefier. A flow diagram of the system is shown in Figure 3.21. 


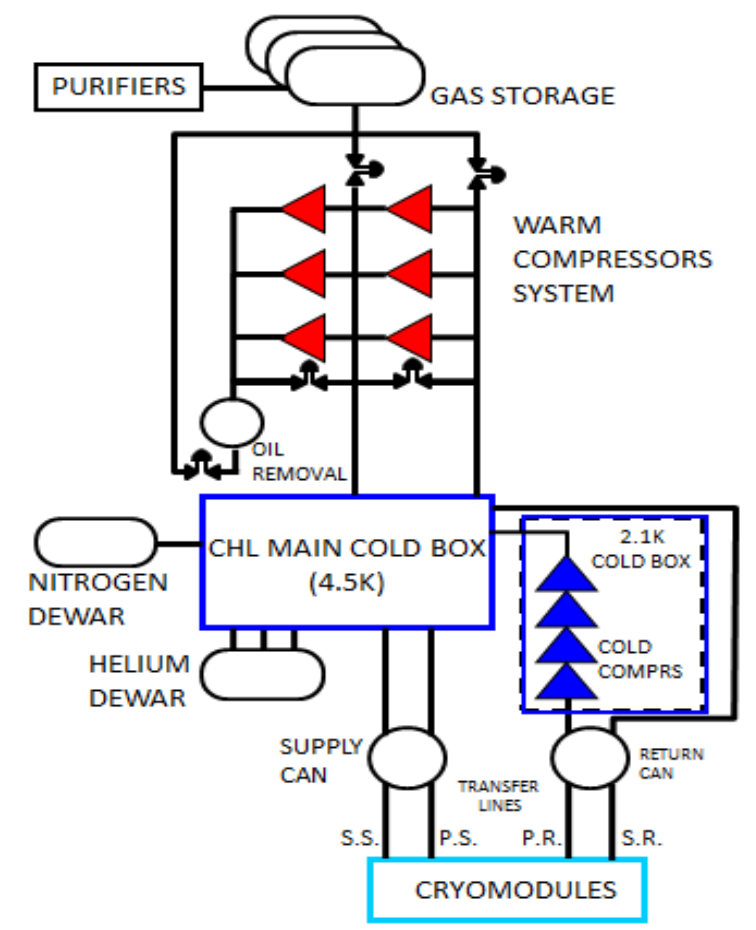

Figure 3.21. Cryogenic system overview.

The control system is an integral part of the SNS cryogenic system. Within the Experimental Physics and Industrial Control System (EPICS), multiple control sequences run to control the operation. These sequences include the $2 \mathrm{~K}$ pump-down, the heater control, the $2 \mathrm{~K}$ trip response, and the JT off sequence. Each time a cryomodule is added to the tunnel, these sequences must be amended to include the new cryomodules. Testing of these sequences will be an essential part of ensuring the success of SCL operation during the PPU project. Perhaps the most critical of these sequences to test is the $2 \mathrm{~K}$ pumpdown sequence. This is an empirically derived sequence that adjusts the gear ratios of the cold compressors as pressure decreases in the return transfer line header. If cryomodules are added to the tunnel, the flow dynamics and temperature profiles within the system could change, requiring development and modification of the $2 \mathrm{~K}$ pump-down sequence.

Because the initial design of the cryogenic system had a large margin of capacity, turn-down studies of the cryogenic system were conducted at SNS to enable the system to run more efficiently. The results of these studies showed that the SNS cold compressors could operate successfully while reducing flow by approximately $25 \%$ from the design flow [18]. Results of the turn-down studies are presented in Table 3.5. Typically, the system is operated with the flow turned town $12.5 \%$ from the design flow. This allows more efficient operation of the system by reducing power consumption and decreasing nitrogen usage while maintaining the robustness of the original design. Even with the system turned down, it is necessary to input approximately $1 \mathrm{~kW}$ of heat into the helium baths of the cryomodules with electric heaters to maintain the minimum flow for the $2 \mathrm{~K}$ cold box. When seven additional cryomodules are added, the turned-down system will be more efficient. The heat load of the additional cryomodules will be less than the heat that is currently added to the system. Therefore, the heater sequence will be adjusted to reduce the external heat presently added, as additional new cryomodules are installed. 
Table 3.5. Results of turn-down study

\begin{tabular}{|c|c|c|c|c|}
\hline & $\begin{array}{c}\text { Design } \\
\text { basis }\end{array}$ & $\begin{array}{l}\text { Maximum } \\
\text { capacity }\end{array}$ & $\begin{array}{l}\text { Nominal } \\
\text { capacity }\end{array}$ & $\begin{array}{r}\text { Minimum } \\
\text { capacity }\end{array}$ \\
\hline First stage compressors $(\mathrm{kW})$ & 608 & & & \\
\hline $\mathrm{C} 1(\mathrm{~kW})$ & & 300 & 250 & 244 \\
\hline $\mathrm{C} 3(\mathrm{~kW})$ & & 300 & 300 & 203 \\
\hline Second stage compressors $(\mathrm{kW})$ & 2074 & & & \\
\hline $\mathrm{C} 4(\mathrm{~kW})$ & & 1456 & 1355 & 762 \\
\hline C5 (kW) & & 1456 & 1355 & 1154 \\
\hline LN2 usage $(\mathrm{g} / \mathrm{s})$ & 120 & 200 & 180 & 150 \\
\hline $\begin{array}{l}\text { Total electric input power to } \\
\text { compressors }(\mathrm{kW})\end{array}$ & 2682 & 3512 & 3260 & 2363 \\
\hline $\begin{array}{l}\text { LN2 equivalent power }(@ 35 \% \\
\text { Carnot) }(\mathrm{kW})\end{array}$ & 216 & 360 & 324 & 270 \\
\hline Total input power equivalent $(\mathrm{kW})$ & 2898 & 3872 & 3584 & 2633 \\
\hline HP to cold box (Atm) & 16.8 & 17 & 16.5 & 12.9 \\
\hline MP cold box out (Atm) & 4 & 2.8 & 2.8 & 2.5 \\
\hline Cold box HP flow (g/s) & 1150 & 1077 & 1030 & 829 \\
\hline CC flow $(\mathrm{g} / \mathrm{s})$ & 125 & 140 & 125 & 90 \\
\hline Liquefaction load $(\mathrm{g} / \mathrm{s})$ & 15 & 4 & 4 & 4 \\
\hline Shield load (kW) & 8300 & 5300 & 5300 & 5300 \\
\hline \multicolumn{5}{|l|}{$\begin{array}{l}\text { Carnot work based on } 2.1 \mathrm{~K} \\
\text { operations }\end{array}$} \\
\hline Primary load (kW) & 400 & 448 & 400 & 288 \\
\hline Liquefaction load (kW) & 102 & 27 & 27 & 27 \\
\hline Shield load (kW) & 61 & 39 & 39 & 39 \\
\hline Total load Carnot work & 563 & 514 & 466 & 354 \\
\hline $\begin{array}{l}\text { Carnot efficiency based on } 2.1 \mathrm{~K} \\
\text { operation }\end{array}$ & 0.194 & 0.133 & 0.130 & 0.134 \\
\hline
\end{tabular}


Table 3.5. Results of turn-down study (continued)

\begin{tabular}{lcccc}
\hline & $\begin{array}{c}\text { Design } \\
\text { basis }\end{array}$ & $\begin{array}{c}\text { Maximum } \\
\text { capacity }\end{array}$ & $\begin{array}{c}\text { Nominal } \\
\text { capacity }\end{array}$ & $\begin{array}{c}\text { Minimum } \\
\text { capacity }\end{array}$ \\
\hline $\begin{array}{l}\text { Carnot work based on 4.5 K } \\
\text { operation }\end{array}$ & & & & \\
$\quad$ Primary load (kW) & 542 & 607 & 542 & 390 \\
$\quad$ Liquefaction load (kW) & 102 & 27 & 27 & 27 \\
$\quad \begin{array}{l}\text { Shield load (kW) } \\
\text { Total load Carnot work }(\mathrm{kW})\end{array}$ & 61 & 39 & 39 & 39 \\
& $\mathbf{7 0 5}$ & $\mathbf{6 7 3}$ & $\mathbf{6 0 8}$ & $\mathbf{4 5 6}$ \\
$\begin{array}{l}\text { Carnot efficiency based on 4.5 K } \\
\text { operation }\end{array}$ & $\mathbf{0 . 2 4 3}$ & $\mathbf{0 . 1 7 4}$ & $\mathbf{0 . 1 7 0}$ & $\mathbf{0 . 1 7 3}$ \\
\hline
\end{tabular}

$\mathrm{HP}=$ high pressure; $\mathrm{MP}=$ medium pressure.

\subsection{SUPPORTING SYSTEMS}

The support systems for the SCL portion of the PPU project include cooling water, beam line vacuum, and insulating vacuum.

The cooling water system will provide cooling to the air-side portion of the FPC inner conductor and is anticipated to be a direct copy of the existing system. Similar to the operation of the original high-beta cryomodules, a water circuit will provide water cooling for four FPCs in series with an RTD at the outlet of each FPC and a flow switch at the return header. The water cooling system is designed to remove a maximum of $200 \mathrm{~W}$ per FPC with a $1 \mathrm{gpm}$ minimum flow. The existing quadrupole magnet cooling system was already verified to meet PPU requirements without modification.

The beam line vacuum system of the PPU cryomodules will be a direct copy of the original SNS cryomodules. Each cryomodule will be equipped with an ion pump that maintains high vacuum. Warm isolation valves will be located at each end of each cryomodule to isolate that section of beam line in the event of a leak. Additionally, these valves will be used to facilitate maintenance on the cryomodules.

Originally, SNS cryomodules were not equipped with an insulating vacuum system. However, leaks were observed on about a third of the cryomodules. Some of these leaks were from the helium circuit to the insulating vacuum, and some were from the air to the insulating vacuum. Individual pump carts were placed on the cryomodules that had leaks. Because of the elevated radiation levels in the tunnel, the controllers for these pumps did not have long lives. A two-turbo molecular pumping system was manifolded to four cryomodules, and the controllers were relocated to the klystron gallery. This system has proved effective. However, it is expensive and has a limited pumping capacity. Recently, a cryomodule positioned in the tunnel beyond the manifolded pumping systems was found to leak. A turbo pump was directly connected to the cryomodule with a backing scroll pump. The controller was located in the klystron gallery away from the radiation environment.

An insulating vacuum system with improved performance over the existing systems is required for the PPU. The new system will consist of turbo-molecular pumps and their corresponding valves and instrumentation directly mounted to each cryomodule, and a centralized roughing pump system backing all of the turbo pumps. Both the turbo-molecular pumps and the roughing system will be controlled through EPICS. By placing the roughing system outside the tunnel, personnel access to the tunnel is minimized and the amount of ancillary equipment located in the tunnel is reduced. This system will also 
include all of the interconnecting piping between the turbo-molecular pump discharge and the roughing pump system.

\subsection{ASSUMPTION LIST}

To prepare the conceptual design approach for the SCL portion of the PPU, the following assumptions were made.

- Plasma processing improves the performance of the medium-beta cavities gradient by an average of $10 \%$.

- A spare medium-beta cryomodule will be available to conduct repairs on underperforming mediumbeta cavities that are not candidates for plasma processing.

- Cavity $11 \mathrm{~b}$ will be repaired in advance of the PPU.

- Cavity 5a will not be repaired in advance of the PPU.

- Available RF power to the original cavities is $550 \mathrm{~kW}$ (current configuration).

\subsection{REFERENCES}

[1] M. Howell, et al., "The First ASME Code stamped cryomodule at SNS," pp. 2465-2467 in Proceedings, 3rd International Particle Accelerator Conference (IPAC2012), New Orleans, May 20-25, 2012.

[2] S. Kim, et al., "The status of the Superconducting Linac and SRF activities at the SNS," pp. 83-88 in Proceedings, 16th International Conference on RF Superconductivity (SRF2013), Paris, France, September 23-27, 2013.

[3] S. Kim, "SNS Superconducting Linac operational experience and upgrade path," pp. 11-15 in Proceedings of 24th International LINAC conference (LINAC08), Victoria, Canada, September 29October 3, 2008.

[4] M. Doleans et al., "Plasma processing to improve the performance of the SNS Superconducting Linac," to be presented at LINAC16, East Lansing, Michigan, October 25-30, 2016.

[5] S. Kim, "Simulation of quench dynamics in SRF cavities under pulsed operation," pp. 1365-1367 in Proceedings of the Particle Accelerator Conference 2003 (PAC2003), Portland, Oregon, May 12-16, 2003.

[6] R. Sundelin, et al., "SNS HOM damping requirements via bunch tracking," pp. 1984-1986 in Proceedings of the 2001 Particle Accelerator Conference (PAC2001), Chicago, June 18-22, 2001.

[7] S. Kim, et al., "Higher-order-mode (HOM) power in elliptical superconducting cavities for intense pulsed proton accelerators," Nuclear Instruments and Methods in Physics Research A, 492(1-2), 110 (October 2002).

[8] S. Kim, I. E. Campisi, D. Jeon, et al., "Study on fault scenarios of coaxial type HOM couplers in SRF cavities," pp. 770-772 in Proceedings, 23rd Annual Conference, Linear Accelerator Conference 2006, Knoxville, Tennessee, August 21-25, 2006. 
[9] Y. Kang, et al., "Electromagnetic simulations and properties of the fundamental power couplers for the SNS superconducting cavities," pp.1122-1124 in Proceedings of the 2001 Particle Accelerator Conference (PAC2001), Chicago, June 18-22, 2001.

[10] W. J. Schneider, et al., "Design of the Spallation Neutron Source (SNS) cryomodule," pp. 11601162 in Proceedings of the 2001 Particle Accelerator Conference (PAC2001), Chicago, June 18-22, 2001.

[11] C. Rode, "The SNS Superconducting Linac system, pp. 619-623 in Proceedings of the 2001 Particle Accelerator Conference (PAC2001), Chicago, June 18-22, 2001.

[12] D.A. Edwards, TESLA Test Facility Linac Design Report, DESY Print TESLA Rep. 95-01, 1995.

[13] J. Preble, I. E. Campisi, E. Daly, et al., "SNS cryomodule performance," pp. 457-461 in Proceedings of the 2003 Particle Accelerator Conference (PAC2003), Portland, Oregon, May 12-16, 2003.

[14] H. Ma, et al., "Low-level RF control of Spallation Neutron Source: System and characterization," Phys. Rev. Special Topics-Accelerators and Beams 9, 032001 (March 2006).

[15] J. Mammosser, "Spallation Neutron Source status and upgrade plans," pp. 62-65 in Proceedings of the 14th International Conference on RF Superconductivity (SRF2009), Berlin, Germany, September 20-25, 2009.

[16] ASME Boiler and Pressure Vessel Code, Section VIII, Division 1-2010 UHA 51(a)(4)(a)(1), American Society of Mechanical Engineers.

[17] F. Casagrande, I. Campisi, P. Gurd, et al., "Status of the cryogenic systems commissioning at SNS," pp. 970-972 in Proceedings of the 2005 Particle Accelerator Conference, Knoxville, Tennessee, May 16-20, 2005.

[18] F. Casagrande, P. Gurd, D. Hatfield, et al., "SNS 2.1 K cold box turn-down studies," pp. 514-516 in Proceedings, 10th European Particle Accelerator Conference (EPAC2006), Edinburgh, Scotland, June 26-30, 2006. 


\section{RADIO FREQUENCY SYSTEMS}

\subsection{HIGH-POWER RADIO FREQUENCY SYSTEMS FOR THE PPU}

The existing SNS linac high-power RF (HPRF) systems are well described by Lynch et al., Champion, and Hardek et al. $[1,2,3]$. The high-level beam parameters for the PPU that drive the linac RF system design considerations are described in Section 2.1. There are two primary impacts to the RF systems: (1) The PPU will require additional SCL HPRF systems to support the additional accelerating structures required to reach a beam energy of $1.3 \mathrm{GeV}$. (2) Modifications will be required to some of the existing normal conducting linac HPRF systems to support the additional beam loading associated with the increase in the average linac beam current.

The SCL high-power systems are described in Section 4.1.1. These are divided into (1) systems supporting newly installed cryomodules and (2) upgrades to systems powering already installed cryomodules. Then upgrades to the high-power systems supporting the already installed lower-energy normal conducting structures are described in Section 4.1.2.

\subsubsection{SCL High-Power Radio Frequency Systems}

\subsubsection{SCL Transmitters}

\section{Existing SCL HPRF Transmitters}

Each of the existing 81 superconducting cavities is driven with RF power from a single $805 \mathrm{MHz}$ klystron. Currently, 70 of the 81 klystrons in use are the model VKP-8291A manufactured by CPI; 9 are the model VKP-8291B manufactured by CPI; and the remaining two are the model TH2169 manufactured by Thales. The VKP-8291A and TH2169 models are specified to produce a peak RF power of $550 \mathrm{~kW}$ and an average power of $49.5 \mathrm{~kW}$. The VKP-8291B is specified to produce a peak RF power of $700 \mathrm{~kW}$ and an average power of $63 \mathrm{~kW}$.

RF power is transmitted via a rectangular waveguide from the output of each klystron, located in the klystron gallery, to the cryomodules located in the accelerator tunnel. The klystrons are protected from reflected power by a waveguide circulator and high-power water load. Each klystron is provided power, utilities, controls, and monitoring by a transmitter. Each transmitter supports a group of 6 klystrons and includes 6 filament power supplies, 12 magnet power supplies, an ion-pump vacuum controller and highvoltage power supply, 6 solid-state RF amplifiers, water cooling and flow monitoring, 2 high-voltage klystron tanks, klystron cathode current monitoring, personnel and equipment safety interlocks, and a programmable logic controller that provides system control. A single HVCM provides a 75 to $80 \mathrm{kV} \mathrm{DC}$ pulse to a grouping of 10 or 11 parallel klystrons spread across multiple transmitters. The new design for the PPU will follow this same general convention, but with slight modifications.

\section{SCL HPRF Transmitters for PPU}

The increase in beam energy for the PPU will require installing an additional $28 \mathrm{RF}$ systems to provide RF power to 28 superconducting accelerating cavities in 7 cryomodules in the SCL. An RF system consists of a single klystron and the associated transmitter components necessary for it to operate. To support 28 klystrons, the PPU will install 5 additional HPRF transmitters to replicate the 6 klystrons per transmitter configuration currently in use at SNS.

Each PPU transmitter will consist of the major components shown in Table 4.1. 
Table 4.1. Major PPU transmitter components

\begin{tabular}{clc}
\hline Item no. & \multicolumn{1}{c}{ Description } & Quantity \\
\hline 1 & Dual filament power supply, 1000VA AC output & 3 \\
2 & Magnet power supply for the klystron gun, 1.2 kW DC output & 6 \\
3 & Magnet power supply for the klystron body, 3.0 kW DC output & 6 \\
4 & Ion pump controller and supply for klystron vacuum, six parallel & 1 \\
& outputs, 5 kV output & 1 \\
5 & Prime AC distribution chassis & 3 \\
6 & Secondary AC distribution chassis & 3 \\
7 & Dual RF amplifier, 805 MHz, 17 W output & 6 \\
8 & Temperature control units for the waveguide circulators & 1 \\
9 & Programmable logic controller & 1 \\
10 & Operator interface & \\
\hline
\end{tabular}

In addition to the ten major items, various circuit boards, flow transmitters, temperature transmitters, AC current transformers, DC current transducers, and other diagnostic components will be included in the complete transmitter system.

To maintain continuity of spare parts across SNS, the new transmitters will be specified to use the same components as the existing ones where possible (see Figure 4.1). For obsolete items, the specification will require that the updated parts be backward-compatible with the existing installation. Any exceptions will be reviewed and explicitly approved by SNS.

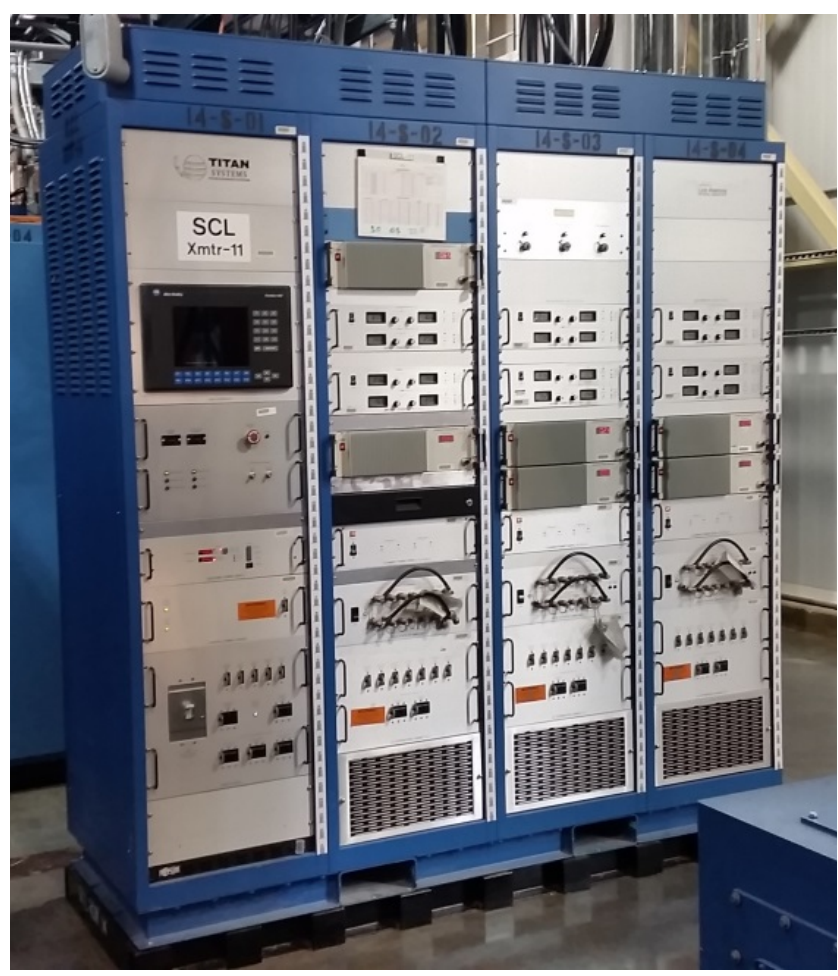

Figure 4.1. The physical layout of PPU RF transmitter racks will be similar to that of the existing racks shown here. 


\subsubsection{SCL Klystrons}

The increase in average linac beam current will require additional RF generator power because of the increase in beam loading. The estimated required RF generator power profile, resulting power margin, and baseline minimum control power margin are shown in Figure 4.2 for the PPU SCL gradient distribution shown in Section 2.1.

The PPU project will use 28 CPI VKP-8291B klystrons. The VKP-8291B klystron operates at $805 \mathrm{MHz}$ and is specified by the manufacturer to produce a peak RF power of $700 \mathrm{~kW}$. Data are available from CPI demonstrating that the klystron is capable of producing up to $1 \mathrm{MW}$ of peak power. The VKP-8291B is physically identical to the VKP-8291A klystron except for minor modifications to cavity tuning, output cavity coupling, and increased cooling requirements to allow operation at the higher output power level.

In addition, the VKP-8291B electron gun is processed at a higher voltage by the manufacturer, so greater cathode voltages are obtainable. The cathodes will operate at a nominal $80 \mathrm{kV}$. The VKP-8219B klystron can also be used as a direct replacement in the existing SCL HPRF klystron sockets. The existing operational transmitters currently include nine VKP-8291B klystrons, although they are operated at the $550 \mathrm{~kW}$ baseline power level.

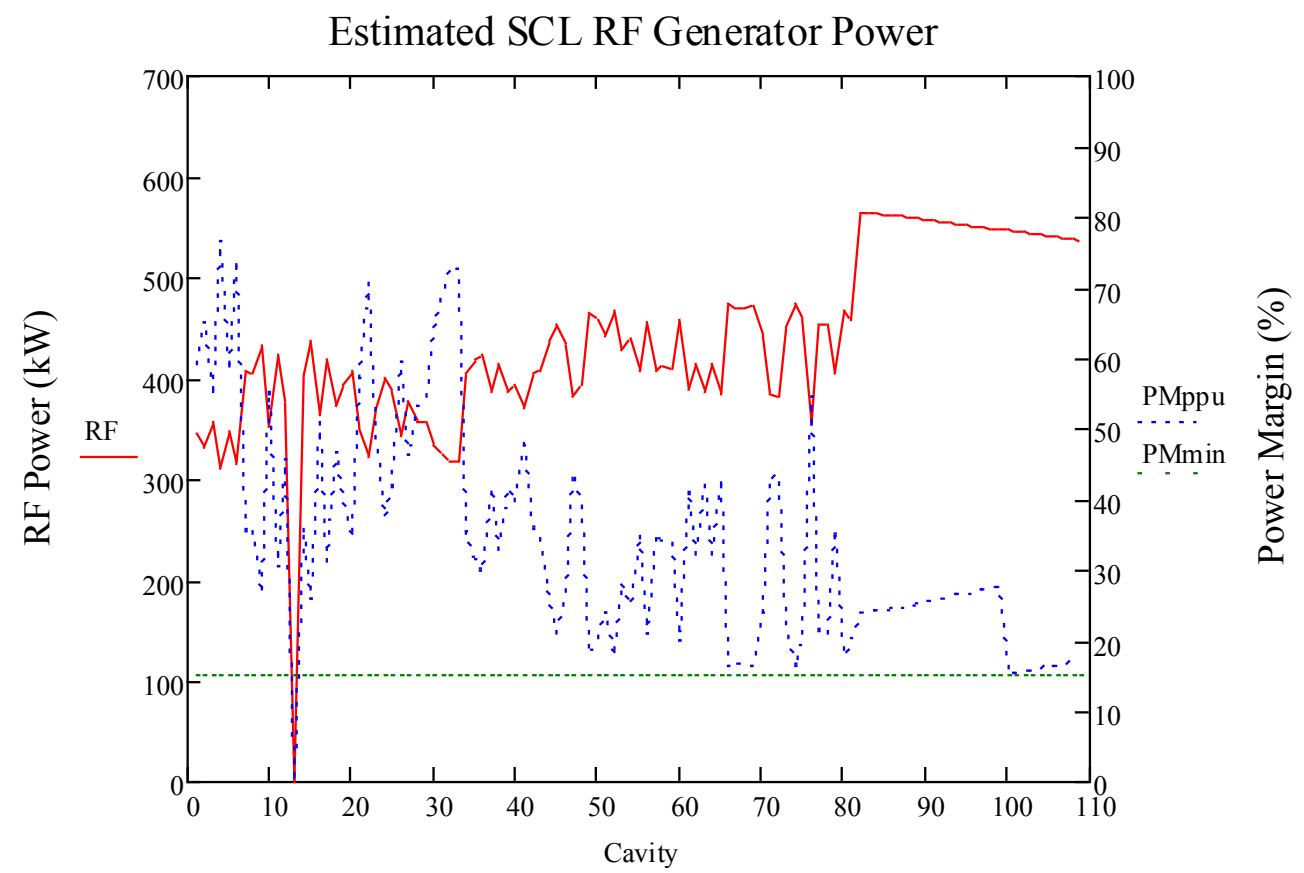

Figure 4.2. Estimated required RF generator power for the SCL.

\subsubsection{SCL Waveguide and Waveguide Components}

The scope of the waveguide runs includes all sections and components from the output elbow of the klystron to the flange on the input coupler of the cryomodule. As in the existing RF transmitters, the waveguide will be a rectangular aluminum WR1150 with flat flanges, and it will not be pressurized (Figure 4.3). The waveguide installation for PPU will be very typical of the existing SCL waveguide at SNS. Components that will be designed, as necessary, into the transmitter waveguide include transitions, straight sections, E-plane sweeps, H-plane sweeps, E-plane miter bends, H-plane miter bends, flexible sections and dual directional power couplers. 


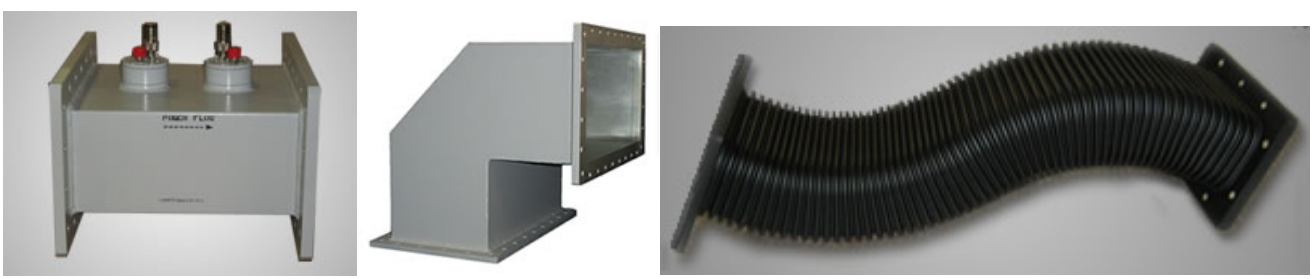

Figure 4.3. Typical waveguide components. MEGA Industries 2016 [4].

The waveguide will be drawn into the 3-dimensional engineering model using the standard sizes, bend radii, and lengths available from major manufacturers. A bill of material will be developed from the model so that all of the waveguides can be procured at one time. An advantage of modeling the waveguide is that modeling can identify and correct interference with other infrastructure before the installation begins.

\subsubsection{SCL Circulators}

In addition to the waveguide components, the PPU design will include high-power waveguide circulators. The circulators will provide a matched load for the output of the klystron by transmitting any reflected power from the RF cavity in the tunnel to a $50 \mathrm{ohm}$ water load. Port one of the circulator will be terminated to the klystron output, port two will be terminated to the waveguide connected to the accelerator cavity, and port three will be terminated to the $50 \mathrm{ohm}$ load. The circulator will be specified to operate in any orientation, upright or inverted.

The circulators will be controlled by biasing solenoids powered from a DC control unit. The control unit will be installed in the transmitter and will power the solenoids based on the input and output temperatures of the circulator cooling water. The return loss of the circulator will be specified to be a minimum of $32 \mathrm{~dB}$, with ports two and three terminated into a matched load and a minimum of $21 \mathrm{~dB}$ or greater across a $24 \mathrm{MHz}$ bandwidth centered on $805 \mathrm{MHz}$. The circulators will be specified for a peak forward power rating of $1 \mathrm{MW}$. An example of the circulator can be seen in Figure 4.4.

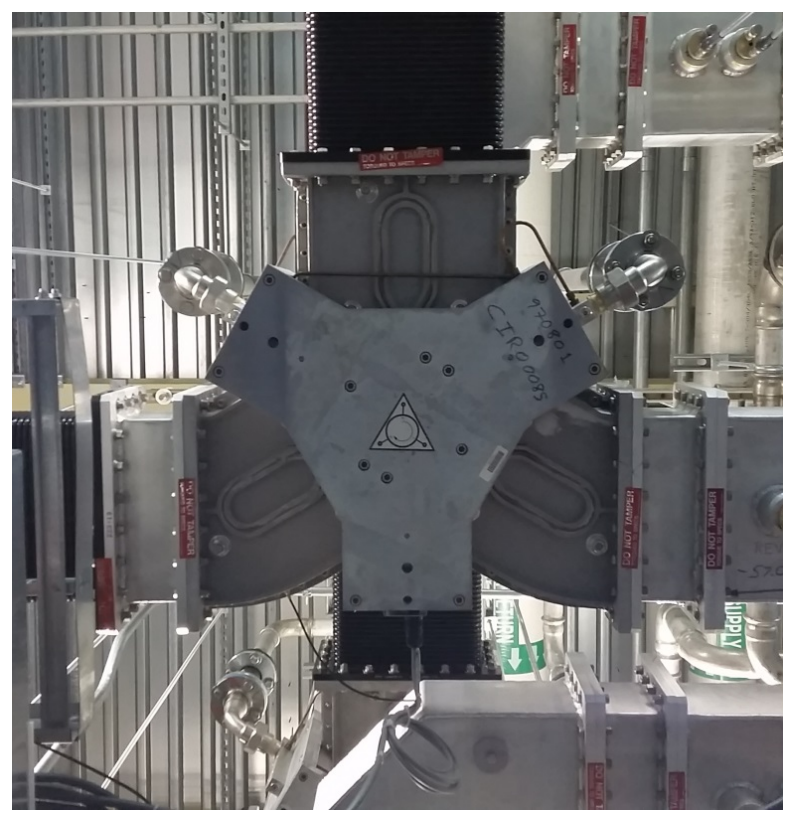

Figure 4.4. High-power waveguide circulator installed in an SCL transmitter at SNS. 


\subsubsection{SCL Water Loads}

Any reflected power from the RF accelerating cavity will dissipate in a $50 \mathrm{ohm}$ water load. The load will be specified for a peak forward power rating of $1 \mathrm{MW}$ and will be terminated to port three of the waveguide circulator to absorb reflected power. The bandwidth of the load will be $\pm 12 \mathrm{MHz}$, centered on $805 \mathrm{MHz}$, and the voltage standing wave ratio (VSWR) will be 1.05:1 or better at $805 \mathrm{MHz}$. The VSWR will not exceed 1.15:1 across the $24 \mathrm{MHz}$ bandwidth. An example of the water load can be seen in Figure 4.5.

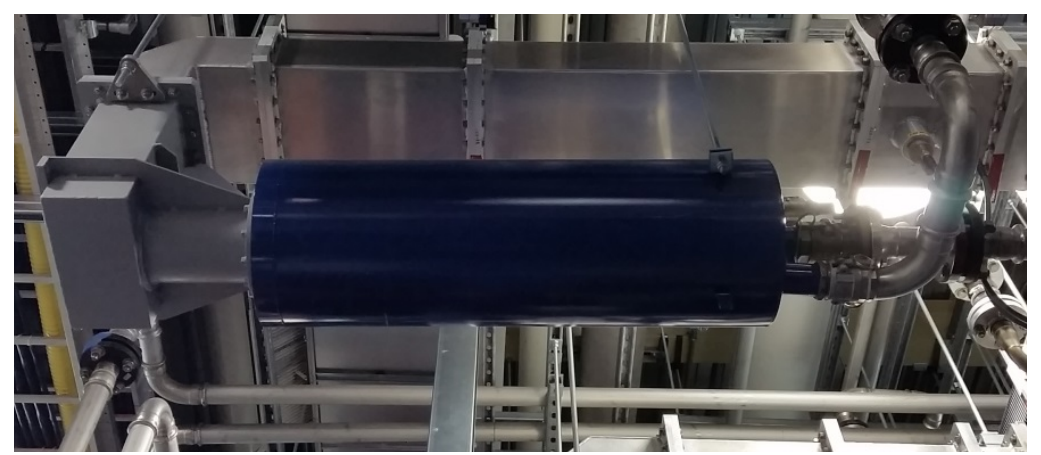

Figure 4.5. Water load installed in an SCL transmitter at SNS.

\subsubsection{SCL HPRF Transmitter Cooling Carts}

The current SCL transmitter cooling cart (TRCC) will be redesigned for the PPU to incorporate ultrasonic flow meters, reduce the physical footprint, and enhance access to waveguide components. In general, each cooling cart will consist of 4 in. diameter stainless steel supply and return piping, which will form the manifold for all of the water-cooled components in each transmitter. The manifold will consist of thirteen 1.5-2 in. stainless steel pipes that provide the supply and return for each component.

As in the existing transmitters, each klystron body will have separate supply and return piping for 6 of the 13 cooling circuits, and the remaining components will be connected in series. Three waveguide circulators will be connected in series with a single supply and return for 2 of 13 circuits. Two collectors will be connected in series for 3 of the 13 circuits, and 3 of the circulator loads will be connected in series on the remaining 2 cooling cart circuits.

The nominal flow requirements for each circuit will be as follows:

- Klystron body-5 gpm

- Klystron collector- 45 gpm

- Circulator-8 gpm

- Circulator load-15 gpm

The existing cooling carts monitor flow only with vortex style in-line meters, but the upgraded cart will be specified to include surface-mounted ultrasonic flow metering with an option to monitor dissipated power. The new flow meters are backward-compatible with the existing TRCCs. Some ultrasonic flow meters are already in use in the SNS transmitters as replacements for failed vortex flow meters.

The TRCCs will be located against the north wall of the klystron gallery. The piping will be designed so that it routes up the north wall and terminates at the associated equipment. The piping will be designed so that it does not obstruct access to RF transmitter components. 
Conceptual layouts of the redesigned cooling cart are shown in Figure 4.6. Item one in the layout is the electronics enclosure. Item three is the TRCC supply header. Items six and seven are the supply and return pipes for transmitter components. Items nine, 10 and 11 are the ultrasonic flow meter and the associated flow transmitter.
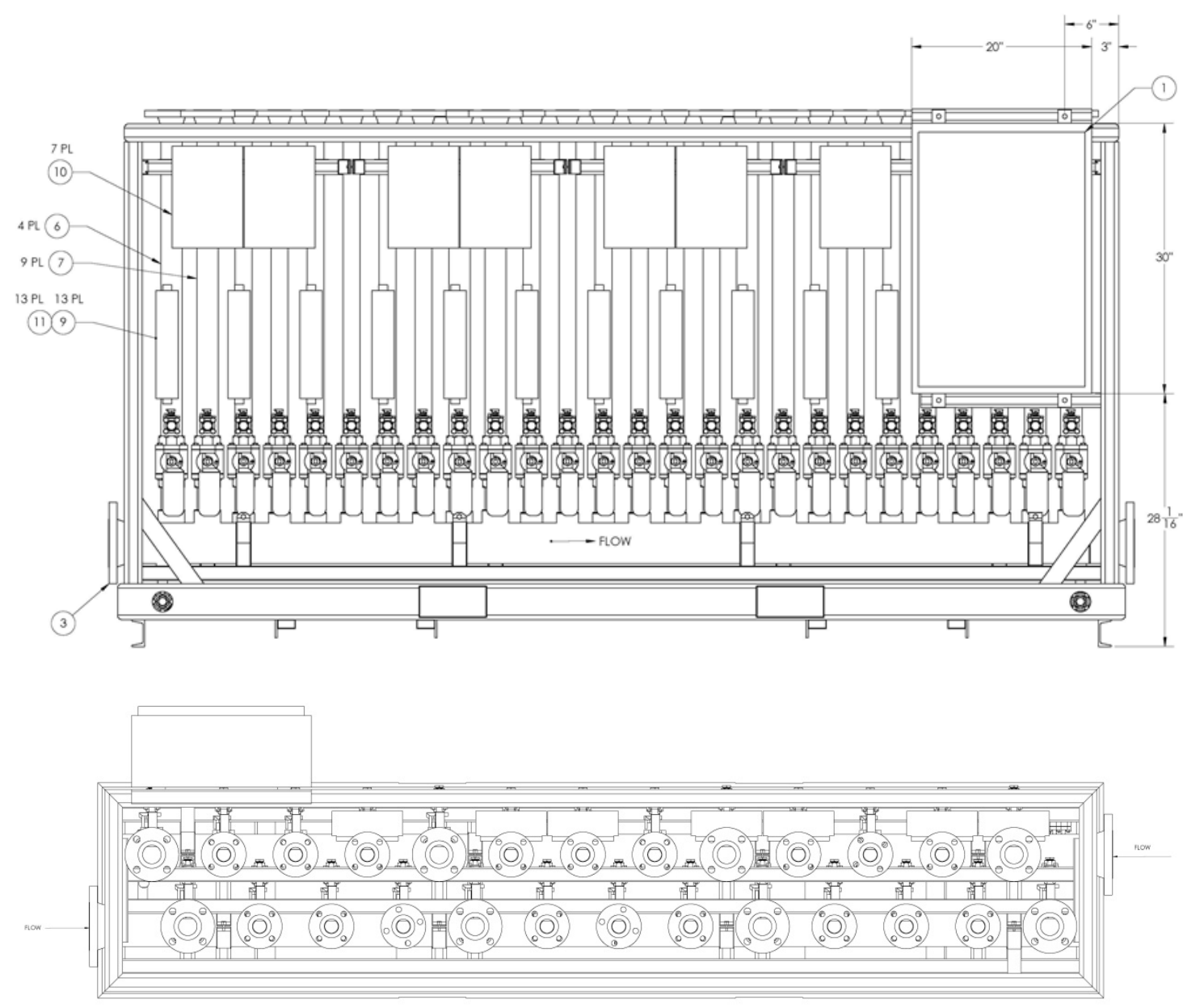

Figure 4.6. Top and side view of the redesigned TRCC.

\subsubsection{Normal Conducting Linac High-Power Radio Frequency Systems}

\subsubsection{Estimated Required RF Generator Power for the Normal Conducting Linac}

The baseline system requirements for the existing normal conducting linac are detailed in a 2000 ORNL report [5]. The design criteria used to size the baseline HPRF systems were based on the estimated required RF generator power for an average linac beam current of $35 \mathrm{~mA}$, calculated using accelerating structure parameters provided by 2 -dimensional cavity simulations. The average linac beam current for the PPU will be increased to $38 \mathrm{~mA}$.

The HPRF power sources were sized to include a minimum power margin to provide available RF power over and above the estimated required RF generator power. The power margin will provide additional available RF power to accommodate losses between the klystron and cavity; variations in klystron and HVCM performance; and the low-level RF(LLRF) phase, amplitude, and resonance control required to maintain steady state operation in response to beam-induced and environmentally induced disturbances. 
Power margin is stated as a percentage of available RF power and is calculated as

$$
\text { Power Margin }(\%)=\left(\frac{\text { Pklystron-Prf }}{\operatorname{Prf}}\right) * 100 \% \text {, }
$$

where

$\mathrm{P}_{\text {klystron }}$ is the rated saturated output power of the klystron

$\mathrm{P}_{\mathrm{rf}}=\mathrm{P}_{\text {copper }}+\mathrm{P}_{\text {beam }}$

\section{Assumptions}

The coupling factor, unloaded Q, external Q, and loaded Q measured on the normal conducting linac accelerating structures during installation (personal communication from C. E. Deibele, ORNL, to M. E. Middendorf, ORNL, July 2014) are summarized in Table 4.2.

Table 4.2. Measured structure parameters for the normal conducting linac

\begin{tabular}{lcccc}
\hline \multicolumn{1}{c}{ Structure } & $\boldsymbol{\beta}_{\text {cpl }}$ & $\mathbf{Q}_{\mathbf{0}}$ & $\mathbf{Q}_{\text {ext }}$ & $\mathbf{Q}_{\mathbf{L}}$ \\
\hline DTL1 & 2.324 & 40886 & 17815 & 12144 \\
DTL2 & 1.445 & 43832 & 30274 & 17947 \\
DTL3 & 1.734 & 48279 & 27697 & 17659 \\
DTL4 & 1.301 & 48118 & 36875 & 20911 \\
DTL5 & 1.277 & 48088 & 37654 & 21129 \\
DTL6 & 1.333 & 48139 & 36056 & 20630 \\
CCL1 & 1.554 & 16000 & 10296 & 6265 \\
CCL2 & 1.184 & 18707 & 15800 & 8565 \\
CCL3 & 1.247 & 19332 & 15503 & 8603 \\
CCL4 & 1.22 & 20890 & 17123 & 9410 \\
\hline
\end{tabular}

$\mathrm{DTL}=$ drift tube linac; $\mathrm{CCL}=$ coupled cavity linac

Recent measured cavity decay time constants agree closely with those calculated from the measured cavity loaded Q values.

Power margins are calculated assuming the baseline RF power sources.

RF power sources for the PPU will have available adequate pulsed DC power; i.e., the HVCM will provide factory-specified klystron cathode voltage and current across the pulse.

Required RF generator power estimates, $P_{g}$, are calculated as

$$
P g=V_{a}^{2} \frac{(1+\beta)}{8 \beta} \frac{1}{r_{L}}\left[\left(1+\frac{I_{g} r_{L}}{V_{a}} \cos \theta\right)^{2}+\left(\tan \varphi+\frac{I_{g} r_{L}}{V_{a}} \sin \varphi\right)^{2}\right]
$$

where

$$
\begin{aligned}
& V_{a}=V_{0} T \\
& \beta=Q_{0} / Q_{\text {ext }} \\
& r_{L}=r_{e} /(1+\beta)
\end{aligned}
$$




$$
\begin{aligned}
& r_{e}=r_{s h} / 2 \\
& \theta=\text { synchronous phase } \\
& \varphi=\text { detuning angle } \\
& I_{g}=2 * I_{b} \text { where } I_{b} \text { is the average linac beam current, } 38 \mathrm{~mA}
\end{aligned}
$$

Equation (2) was used to calculate the RF power requirements as listed in columns 2-4 of Table 4.3. Already it can be seen that the power margin for DTL4 and DTL5 is less than 20\%. However, based on results of recent studies during accelerator physics shifts, it was decided to compare the calculations with the actual operating parameters of the SNS linac. Two data sets were examined, one from 2014 and the other from 2016. They were self-consistent within a few percentage points; their mean is presented in column 6 of Table 4.3. These data represent operating power levels scaled to $38 \mathrm{~mA}$. Notice that the measured data are consistently greater than the calculated results by a factor of approximately 1.1 , with a commensurate decrease in power margin. Note that power margin calculations assume each klystron is capable of its rated saturated power of $2.5 \mathrm{MW}$ and 5.0 MW for the DTL and CCL, respectively. This assumption is presently invalid because the existing HVCMs cannot drive the klystrons at their full rated power levels.

Table 4.3. Estimated required RF generator power for the normal conducting linac

\begin{tabular}{lcccccc}
\hline Structure & $\begin{array}{c}P_{\text {copper }} \\
(\mathrm{kW})\end{array}$ & $\begin{array}{c}\text { Pbeam }_{(\mathrm{kW})} \\
\text { DTL1 }\end{array}$ & $\begin{array}{c}\mathrm{P}_{\text {total }} \\
(\mathrm{kW})\end{array}$ & $\begin{array}{c}\text { PM } \\
(\%)\end{array}$ & $\begin{array}{c}\text { Measured } \\
(\mathrm{kW})\end{array}$ & $\begin{array}{c}\text { Measured PM } \\
(\%)\end{array}$ \\
DTL2 & 985 & 201 & 555 & 350 & 636 & 293 \\
DTL3 & 1238 & 725 & 1710 & 46 & 1983 & 26 \\
DTL4 & 1239 & 893 & 2003 & 25 & 2141 & 17 \\
DTL5 & 1258 & 878 & 2136 & 17 & 2306 & 10 \\
DTL6 & 1191 & 763 & 1954 & 28 & 2109 & 8 \\
CCL1 & 2203 & 1002 & 3205 & 56 & 3747 & 33 \\
CCL2 & 2245 & 1242 & 3487 & 43 & 4065 & 23 \\
CCL3 & 2340 & 1328 & 3667 & 36 & 4176 & 20 \\
CCL4 & 2332 & 1395 & 3727 & 34 & 4105 & 22 \\
\hline
\end{tabular}

Each structure of the drift-tube linac (DTL) is currently driven with RF power from a single $402.5 \mathrm{MHz}$, $2.5 \mathrm{MW}_{\mathrm{pk}}\left(200 \mathrm{~kW}_{\mathrm{ave}}\right)$ klystron. Each klystron is supported by a single transmitter that provides power, utilities, monitoring, control, and personnel and equipment protection. A single HVCM provides pulsed dc power to two DTL klystrons. The RF power is transmitted via a WR2 100 rectangular waveguide from the output of the klystron - located in the klystron gallery - to an RF vacuum window, ridge-loaded waveguide taper, and coupling iris on the DTL tank located in the accelerator tunnel. Each klystron is protected from reflected power by a waveguide circulator with a water-/glycol-cooled load. There are a total of three DTL HVCMs; six DTL high-voltage tanks with klystrons; six circulators with circulator loads; and six waveguide runs, RF vacuum windows, tapers, and coupling irises for the six DTL accelerating structures.

For the coupled-cavity linac (CCL), RF power from a single $805 \mathrm{MHz}, 5 \mathrm{MW}_{\mathrm{pk}}\left(400 \mathrm{~kW}_{\text {ave }}\right.$ ) klystron is divided equally and drives bridge couplers at two locations on each structure. Each klystron is supported by a single transmitter that provides power, utilities, monitoring, control, and personnel and equipment protection. A single HVCM provides pulsed dc power to a single CCL klystron. Each klystron is protected from reflected power by a waveguide circulator with a water-cooled load. The RF power is transmitted via a single WR1150 rectangular waveguide from the output of the klystron/circulator to the 
$3 \mathrm{~dB}$ hybrid power divider, located in the klystron gallery. RF power is divided equally and transmitted via two WR1150 waveguide runs to RF vacuum windows and bridge couplers on the appropriate CCL tank located in the accelerator tunnel. There are a total of four CCL HVCMs, four CCL high-voltage tanks with klystrons, four circulators with circulator loads, four $3 \mathrm{~dB}$ waveguide hybrid power dividers with water-cooled loads, and eight waveguide runs and RF vacuum windows.

\subsubsection{Normal Conducting Linac HPRF Summary}

Assuming factory-specified cathode voltage and current across the HVCM pulse, estimates of RF power required by the DTL suggest that the DTL4 and DTL5 klystrons will be operating with unacceptably low power margins. In addition, the power margins for DTL3, DTL6, and CCL2-3 are not generous and may prevent stable and reliable operation of the linac at $38 \mathrm{~mA}$. Further studies are planned in the near future to improve understanding of the RF power requirements as compared with existing capabilities. The results of these studies may indicate the need for upgrades to the HVCMs in the normal conducting linac.

The increase in the average linac beam current to $38 \mathrm{~mA}$ will require modifying the DTL4 and DTL5 HPRF systems to accommodate a $3 \mathrm{MW}_{\mathrm{pk}}\left(240 \mathrm{~kW}_{\text {ave }}\right)$ klystron. Because of the current configuration of two paired DTL klystrons per HVCM, this will require recabling DTL3 and DTL6 together on one HVCM, and DTL4 and DTL5 on a modified HVCM. The DTL4 and DTL5 high-power circulators and water loads will also be upgraded to accommodate the increase in RF power.

Integrating a $3 \mathrm{MW}_{\mathrm{pk}}\left(240 \mathrm{~kW}_{\mathrm{ave}}\right)$ klystron into the current transmitter configuration is anticipated to be straightforward, but it may require upgrading the filament and magnet power supplies. In addition, the increase to $3 \mathrm{MW}_{\mathrm{pk}}\left(240 \mathrm{~kW}_{\mathrm{ave}}\right) \mathrm{RF}$ power would require additional cooling to remove an estimated maximum incremental increase of $\sim 80 \mathrm{~kW}_{\text {ave }}$ per klystron in collector power (no RF, $7.4 \%$ duty factor).

Table 4.4 summarizes the baseline warm linac RF equipment and the additional required RF equipment to support the PPU.

Table 4.4. Normal conducting linac high-power RF systems for the PPU

\begin{tabular}{lccc}
\hline \multicolumn{1}{c}{ Major equipment } & Baseline & PPU upgrade & Final configuration \\
\hline DTL klystron & $6(2.5 \mathrm{MW})$ & Upgrade 2 sockets to 3 MW & $4(2.5 \mathrm{MW}), 2(3 \mathrm{MW})$ \\
DTL transmitter & 6 & Modify to support 3 MW tube & $4(2.5 \mathrm{MW}), 2(3 \mathrm{MW})$ \\
DTL circulator & 6 & Upgrade to support 3 MW tube & $4(2.5 \mathrm{MW}), 2(3 \mathrm{MW})$ \\
DTL circulator load & 6 & Upgrade to support 3 MW tube & $4(2.5 \mathrm{MW}), 2(3 \mathrm{MW})$ \\
CCL klystron & $4(5 \mathrm{MW})$ & - & $4(5 \mathrm{MW})$ \\
CCL transmitter & 4 & - & 4 \\
\hline
\end{tabular}

\subsection{LOW-LEVEL RADIO FREQUENCY SYSTEMS}

\subsubsection{System Overview}

The LLRF systems provide low-power RF drive to the linac HPRF systems and, most important, control the phase and amplitude of each cavity. The initial baseline stability requirements for the control are $\pm 1.0^{\circ}$ in phase and $\pm 1.0 \%$ in amplitude. The LLRF control systems on the 96 existing systems exceed this specification, with \pm 0.5 degrees in phase and $\pm 0.5 \%$ in amplitude routinely observed. A block diagram of the LLRF system is shown in Figure 4.7. 


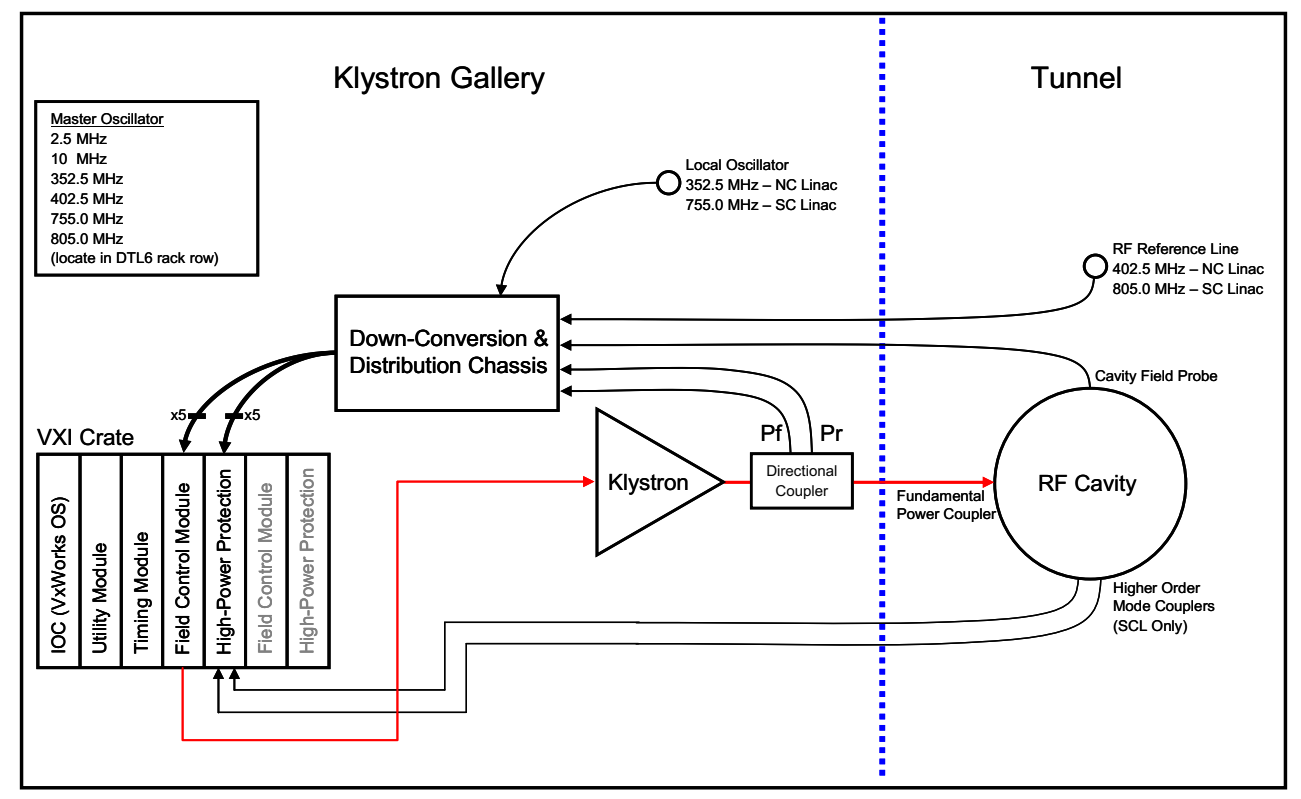

Figure 4.7. Block diagram of the SNS low-level control system.

The existing LLRF system is a digital control system that fundamentally realizes a proportional-integral (PI) feedback controller along with AFF to support cavity filling and beam loading conditions. The heart of the system is the field control module (FCM), which digitizes four analog input channels (cavity field, reference, forward, and reflected signals), digitally processes the data stream, and produces an output signal at either 402.5 or $805 \mathrm{MHz}$, depending on the location in the linac. External communication is achieved via the slot-zero input-output controller (IOC) running the VxWorks operating system. The LLRF finite state machine is implemented as an EPICS sequencer running on the IOC. The LLRF control system also provides high-power protection via the high-power protection module, which provides for fast shutdown of the drive in case of overpower; arcs in the distribution system; poor vacuum; or "soft" interlocks such as cryo, coupler cooling, and HPRF permit. Down-conversion of reference and cavity signals is performed in a temperature-regulated chassis. The master oscillator provides low-noise, phasecoherent reference signals that are distributed throughout the klystron gallery and tunnel [6].

Although the existing LLRF system meets the requirements for the PPU project, for several reasons, the system is not the ideal solution for the PPU project. The following are the known limitations of the system:

- Several key components (including the field-programmable gate arrays [FPGAs]) are obsolete and cannot be acquired, so additional modules cannot be fabricated.

- The current system's VXI backplane bus bandwidth limits the update rate from the IOC to the LLRF hardware to $20 \mathrm{~Hz}$, which will not allow for pulse-to-pulse AFF correction and the desired improvement in the learning times of the system.

- For the STS, a second beam loading feed-forward buffer will be required to meet specifications, and the current hardware is inadequate to support this feature.

For these reasons, it is necessary to implement a redesigned LLRF system for both the PPU and STS projects. 


\subsubsection{SCL LLRF Systems for PPU}

The increase in beam energy for the PPU will require installing an additional 28 LLRF systems to provide RF power to 28 superconducting accelerating cavities in 7 high-beta cryomodules in the SCL. The replacement LLRF systems for the PPU will be installed with two systems per crate in a single LLRF rack, grouped in a three-rack layout to support six cavities. This layout will maintain the pattern used for the initial SNS installation. Figure 4.8 shows the physical layout of the LLRF racks.

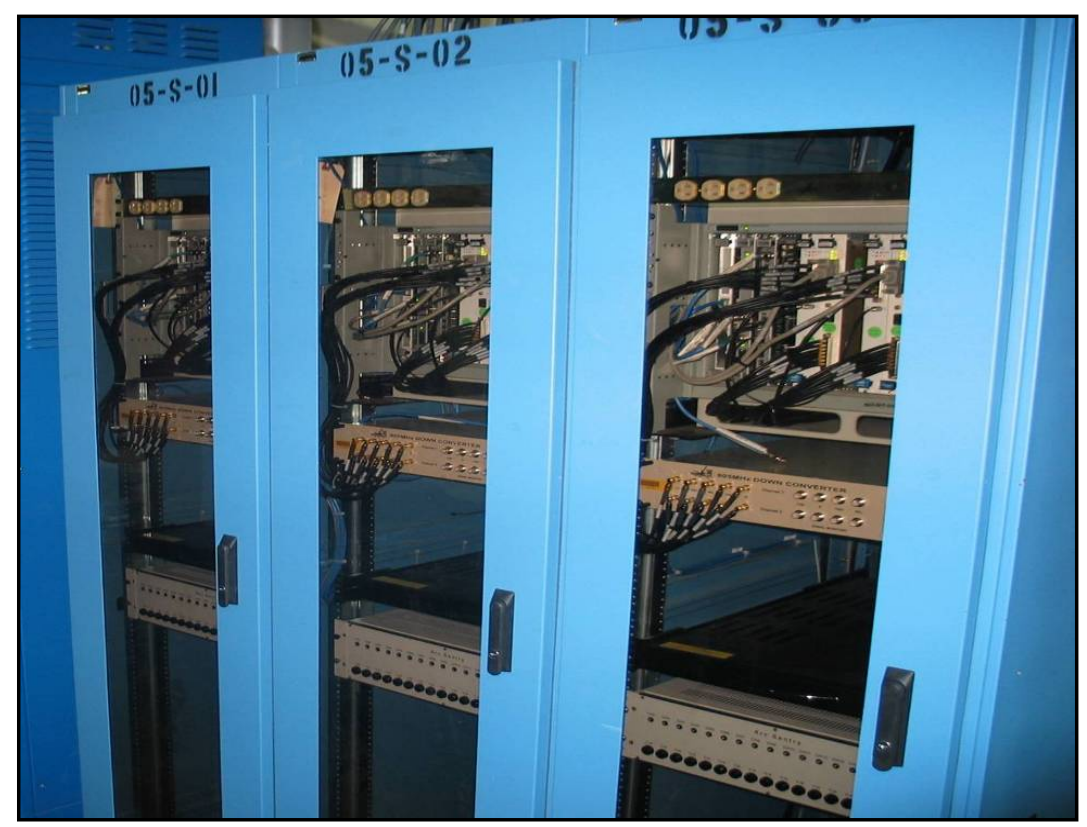

Figure 4.8. The physical layout of the PPU LLRF racks will be similar to that of the existing racks, shown here.

The plan for the PPU is to develop a $\mu$ TCA.4-based LLRF system capable of supporting the full STS requirements. This technology will support PCIe-based backplane communication that will provide adequate bandwidth to easily support $60 \mathrm{~Hz}$ AFF operation as well as potential future upgrades that may be desired. It will be similar to the current LLRF system in that it is crate-based with two LLRF control systems installed per crate. The crate will also support the IOC and the timing module. To limit the development time, commercial components will be used where possible. The major hardware components that will be purchased are shown in Table 4.5. The quantities listed will support two LLRF systems.

Table 4.5. Commercially available components

\begin{tabular}{clc}
\hline Item no. & \multicolumn{1}{c}{ Description } & Quantity \\
\hline 1 & $\mu$ TCA.4 chassis with 12 AMC slots and 1000 W power module & 1 \\
2 & $\mu$ TCA MCH module and shelf manager & 1 \\
3 & AMC502 FPGA carrier with dual FMC slots & 2 \\
4 & AMC 726-core I-7 processor & 1 \\
5 & AMC FPGA carrier with timing FMC card & 1 \\
\hline
\end{tabular}

Several major pieces of the LLRF system cannot be purchased and will require design and development effort. For the replacement FCM, a commercial AMC FPGA carrier card will be used for the main portion of the board (AMC502), but dedicated FMC analog-to-digital converter and digital-to-analog converter 
modules will need to be developed. To replace the protection features, a new high-power protection module will also need to be designed. For the new system, the current $50 \mathrm{MHz}$ intermediate frequency will be retained so that the installed reference line and local oscillator distribution can be maintained.

The present dual down-converter chassis will be redesigned to include all frequency conversion functions; this will simplify the design of the FCM and allow for improved temperature stability of the overall system by temperature-compensating all high-frequency components. This chassis will be similar to the chassis that was developed for the $50 \mathrm{MHz}$ FCM temperature dependence studies in 2013 [7].

Because of the new hardware, the current firmware will need to be replaced to support the added features. The firmware used in the original FCM is based on under-sampling using a five-over-four routine that allows for $\backslash \backslash \mathrm{Q}$ signals to be obtained. Plans are for the new firmware to use oversampling techniques that will improve regulation and off-frequency operation for the warm linac. Plans also include increasing the buffer sizes to support decreased granularity of the feed-forward system to achieve tighter overall regulation. Finally, improved AFF learning techniques will need to be implemented to support faster correction times and a second buffer set to allow for dual beam types. The second buffer is not required for the PPU, and the development effort for this feature will be deferred until the STS project is approved.

One of the reasons to choose $\mu$ TCA for the LLRF platform is that the Controls Group will use this platform for its future development and will support the new IOCs. This will reduce the development effort for the underlying software drivers for the system and allow the effort to be placed at the application level. The plan is to maintain the current look and feel of the operational LLRF EPICS screens to minimize the learning curve for the operation staff. Doing so will require modifying the existing LLRF database to support the new memory map that will be developed to support the hardware. It will also require modifying the existing EPICS screens to support the additional hardware features and properly map to the new database.

\subsubsection{Arc Detectors}

The arc detector system, a commercial product provided by Advanced Ferrite Technology, is used throughout the SNS installation. The system will be used for the PPU and can support up to 16 arc detector channels per chassis. It routes light from five test points located in the RF distribution system (vacuum and air sides of the RF cavity windows or couplers, the RF circulators, the RF circulator loads, and the klystron windows) via fiber optic cables. Radiation-hardened plastic fibers are used in highradiation areas to prevent darkening of the fibers. An arc test system is used to verify the integrity of critical fiber paths daily to ensure the functionality of the system. The continued use of this system will minimize the number of spares required to support operations. Figure 4.9 is an image of the Advanced Ferrite Technology chassis and one of the arc detector cards. The nominal SCL system contains two banks of five arc detector cards and a shared dual output interface card that serves two cavities. 

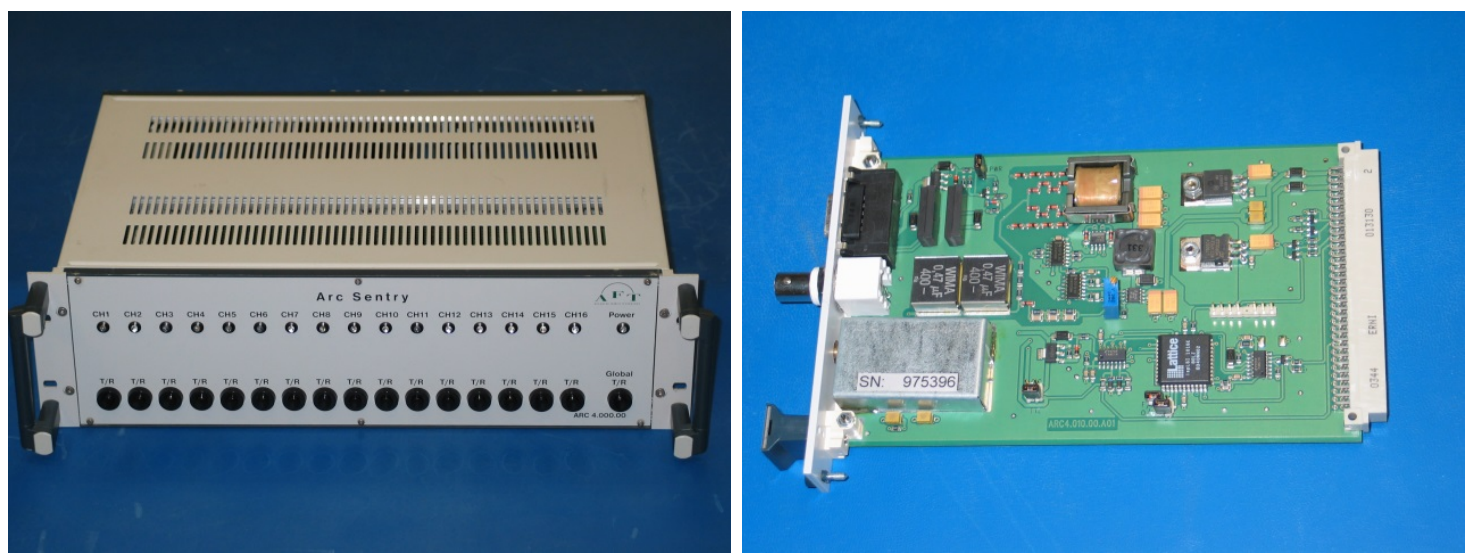

Figure 4.9. Advanced Ferrite Technology arc detector system.

\subsubsection{Reference Line}

The SNS RF reference system includes the master oscillator, the local oscillator (LO) distribution system, and the reference RF distribution system (see Figure 4.10). The master oscillator generates six continuous wave (CW) coherent low-noise output signals at 2.5, 10, 352.5, 402.5, 755, and $805 \mathrm{MHz}$. These RF signals provide the capability to control the phase relationships between the fields in the front-end and linac RF cavity structures [8].

The $805 \mathrm{MHz}$ RF reference distribution system is installed through the end of the linac tunnel, and no additional installation is required for the PPU project. The $755 \mathrm{MHz}$ LO distribution will require one additional directional coupler in the existing primary $755 \mathrm{MHz}$ heliax distribution line, one additional LO distribution amplifier, and heliax distribution cables going to the PPU LLRF rack groups.
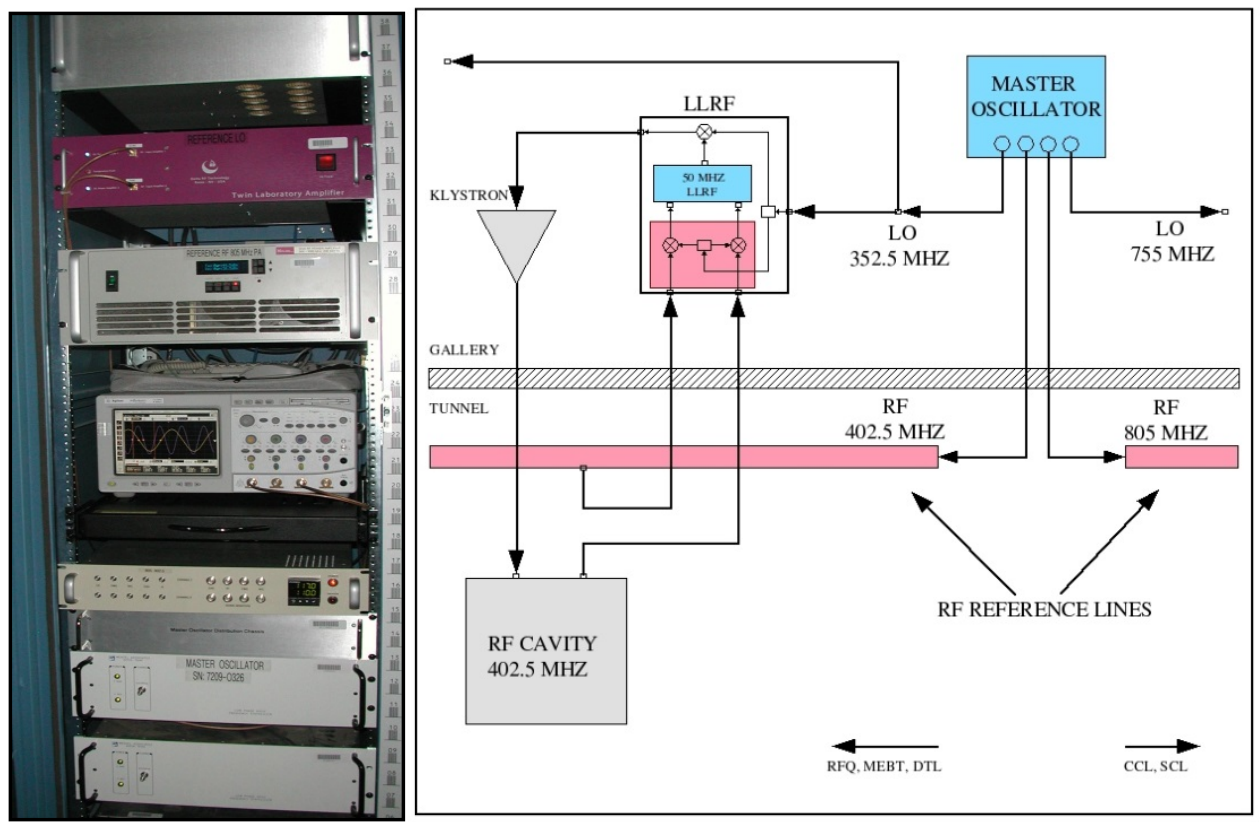

Figure 4.10. Master oscillator rack and block diagram. 


\subsection{MODULATOR CONSIDERATIONS}

\subsubsection{System Overview}

The HVCM uses AC power from the $13.8 \mathrm{kV}$ utility feeds and coverts it into high-voltage pulses used to drive high-power klystrons [9]. Since there are basically three different types of klystrons, operating at different frequencies and power levels, the modulator is configured differently in the DTL, CCL, and SCL sections of the linac. Using different ratios of klystrons to modulators allows all modulators to operate at essentially the same power level, but the different voltage requirements mandate slightly different component selections for the HVCM systems. This results in a minimum number of spare parts and minimizes the training required to support and maintain the modulator systems.

A basic modulator system-level block diagram is shown in Figure 4.11, along with photographs of the major subsystems in Figure 4.12. The $13.8 \mathrm{kV} 3$-phase AC is transformed down to $2100 \mathrm{~V}$ after passing through 5th and 7th harmonic filters. The AC is rectified, using silicon-controlled rectifiers (SCRs), to DC voltages of up to $\pm 1300 \mathrm{~V}$. Two large capacitor banks store up to $200 \mathrm{~kJ}$ of energy. The DC bus feeds three IGBT H-bridge "switch plates" that chop the DC up at $20 \mathrm{kHz}$. This system supplies power to the primaries of nanocrystalline core step-up transformers submerged in an oil-filled tank. The outputs of the transformers are then rectified to produce DC, and the three phases are combined to generate the highvoltage pulses. After passing through a $\pi$ filter to remove some of the high-frequency content, the pulses are transmitted down a triaxial cable to the klystron load(s).

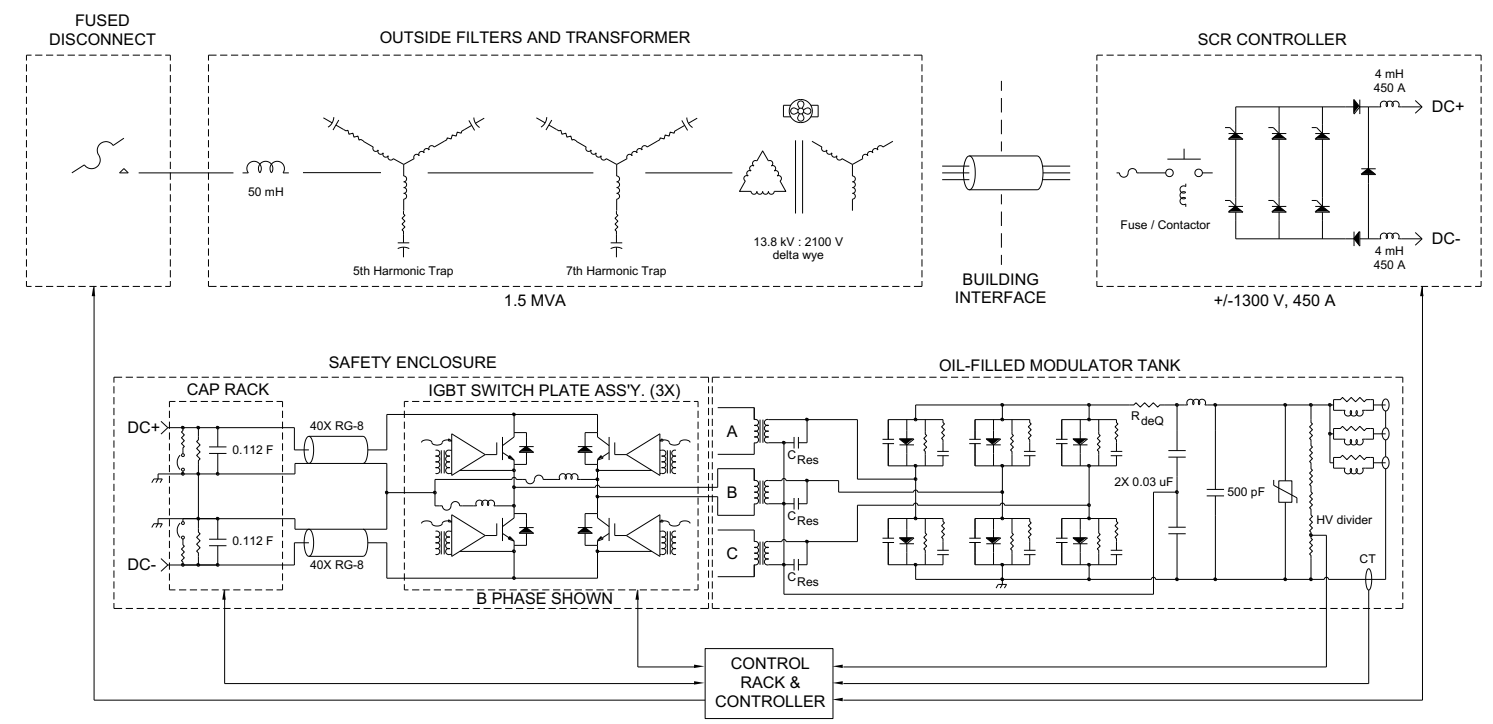

Figure 4.11. Block diagram of the HVCM system. 


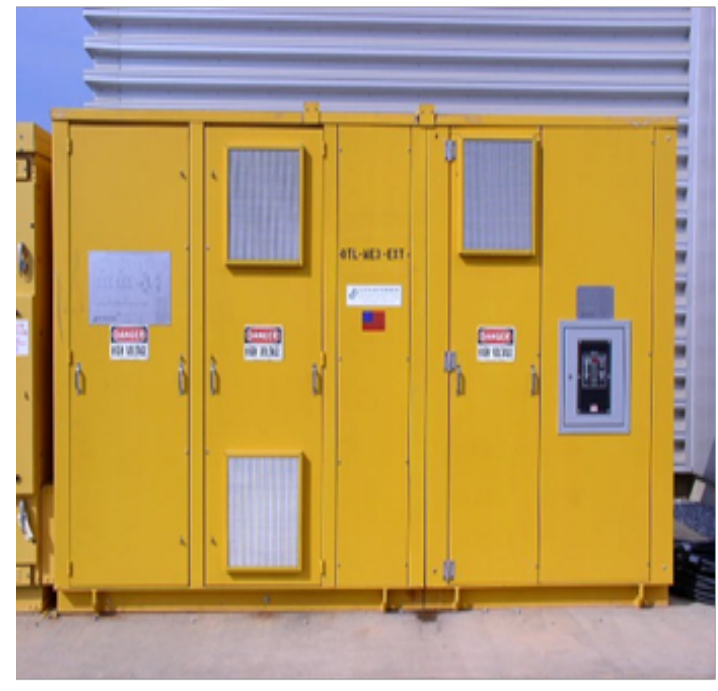

Outside $13.8 \mathrm{kV}: 2100 \mathrm{~V}$

1.5 MVA transformer

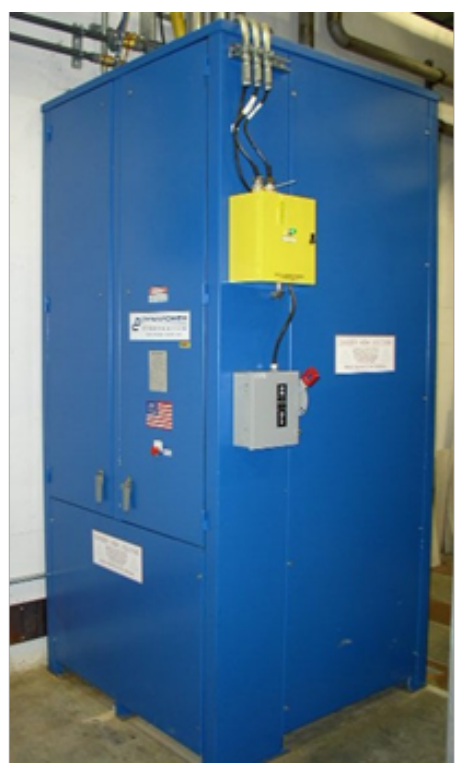

SCR controller

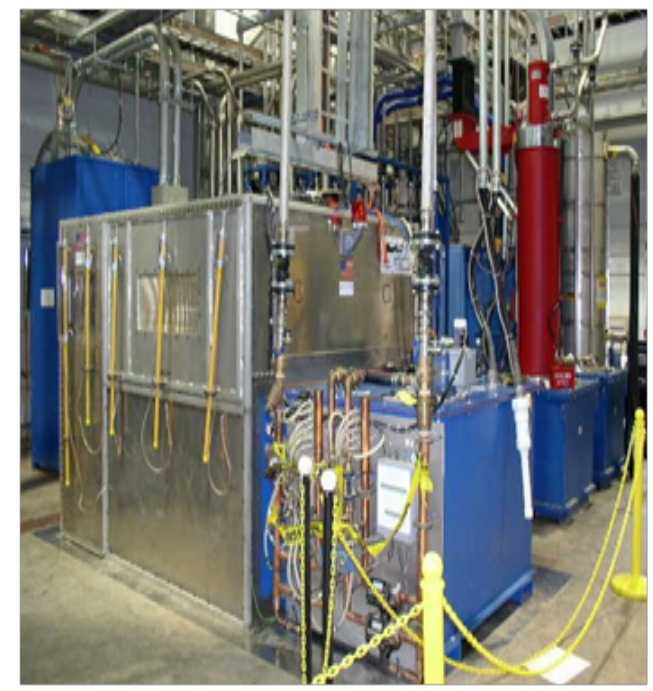

Safety enclosure and oil-filled modulator tank

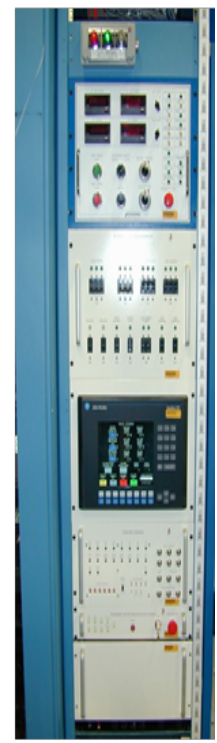

Controller rack

Figure 4.12. Major components in the HVCM system.

\subsubsection{System Requirements and Interfaces}

The modulator design for the PPU involves several tradeoffs necessary to ensure that system reliability is at least equivalent to the current HVCM operational performance. The system requirements are dictated by the cavity power requirements and the klystron performance characteristics, which are defined in Section 4.1. The increased and/or new cavity power requirements demand the following from the modulator systems:

- Increased operating voltage levels to drive the $700 \mathrm{~kW}$ klystrons supplying the 28 new cavities. 
- Increased operating voltage levels to allow the existing SCL cavities to operate above present levels.

- Increased operating voltage levels in the warm section of the linac. This is a marginal increase and can be accommodated with the present systems without any modifications, with the exception of the DTL 4 and 5 klystrons.

- Increased operating voltage levels in the warm section of the linac to support the higher beam current loading and supply adequate power to the two new 3.0 MW, 402.5 MHz DTL klystrons.

Each of these situations will be considered separately in the following subsections. Wherever possible, the modulators will be similar to the existing HVCMs to minimize spare parts inventories, optimize operational efficiency, and minimize the amount of training necessary to support modulator operation. Table 4.6 summarizes the major parameters for the new and upgraded current modulator systems required to support the PPU.

Table 4.6. Present and projected operating parameters for existing and new HVCM systems

\begin{tabular}{lccccc}
\hline HVCM system & $\begin{array}{c}\text { Present output } \\
\text { voltage } \\
(\mathbf{k V})\end{array}$ & $\begin{array}{c}\text { Present } \\
\text { output } \\
\text { current } \\
(\mathbf{A})\end{array}$ & $\begin{array}{c}\text { PPU output } \\
\text { voltage } \\
(\mathbf{k V})\end{array}$ & $\begin{array}{c}\text { PPU output } \\
\text { current } \\
\text { (A) }\end{array}$ & $\begin{array}{c}\text { PPU modulator } \\
\text { average power } \\
\mathbf{( k W )}\end{array}$ \\
\hline Existing DTL HVCMs & -125 & 66.9 & -126 & $85^{1}$ & 804 \\
New 3.0 MW DTL HVCM & N/A & N/A & -124 & 78.4 & 786 \\
Existing CCL HVCMs & -132 & 69.8 & -132 & 70.0 & 748 \\
Existing SCL HVCMs & -74 & 116 & -77 & 121 & 755 \\
New SCL HVCMs & N/A & N/A & -81.7 & 123.2 & 803 \\
\hline
\end{tabular}

${ }^{1}$ Calculated output current of RFQ-Mod1, which operates at reduced voltage levels.

Modulator tradeoffs primarily revolve around the main active component of the system, the insulated gate bipolar transistor (IGBT) switches. Currently, 3300 V, 1200 A IGBT switches are used because they represent the highest-power modules available that are capable of operating reliably at $20 \mathrm{kHz}$. Other higher-power switches have been studied throughout the years of HVCM development, but they either have unacceptably high switching losses or long delay and/or transition times in turning on or off. Since no external data exist for these switches at the chosen switching frequency, the reliability of the power electronics is based on operational experience and engineering scaling.

The SNS HVCM IGBTs are able to operate reliably at 70\% of their voltage rating without snubbers and at $75 \%$ of their rating, at least, with snubbers. Experience has shown that turning off an IGBT while it is conducting $1 \mathrm{kA}$ or greater is detrimental to its long-term durability. Likewise, maintaining switching losses below approximately $5 \mathrm{~kW}$ is desirable to maintain system reliability. It is also desirable to maintain these operating limits so as to permit the use of existing SCR controllers and $13.8 \mathrm{kV}: 2100 \mathrm{~V}$ dry cast distribution transformers. Some additional HVCM requirements are listed in Table 4.7. 
Table 4.7. HVCM requirements for new and existing systems

\begin{tabular}{|c|c|}
\hline Parameter & Value \\
\hline Input voltage, AC RMS & $13.8 \mathrm{kV} \pm 3 \%, 3$ phase \\
\hline Input $\mathrm{AC}$ apparent power & $1.5 \mathrm{MVA}$ \\
\hline $161 \mathrm{kV}: 13.8 \mathrm{kV}$ transformer rating & 25/33/41.6 (OA/FA/FA) MVA \\
\hline Source impedance of $161 \mathrm{kV}: 13.8 \mathrm{kV}$ transformer & $8-10 \%$ \\
\hline Permissible harmonic generation & IEEE Std 519 \\
\hline Maximum power factor & 0.95 \\
\hline Maximum pulse repetition frequency & $60 \mathrm{~Hz}$ \\
\hline Pulse width & $0.3-1.5 \mathrm{~ms}$ \\
\hline Maximum output voltage & $110 \%$ of Table 1.1 .1 , negative polarity \\
\hline Maximum output current & $110 \%$ of Table 1.1 .1 \\
\hline Maximum rise time, $0-90 \%$ & $125 \mu \mathrm{s}$ \\
\hline Maximum fall time, $90-10 \%$ & $100 \mu \mathrm{s}$ \\
\hline Maximum droop & $1 \%$ \\
\hline Maximum ripple & $1 \%$ \\
\hline Maximum fault (short-circuit) energy & $20 \mathrm{~J}$ \\
\hline $\begin{array}{l}\text { Maximum response time to detected high-speed } \\
\text { fault }\end{array}$ & $2 \mu \mathrm{s}$ \\
\hline $\begin{array}{l}\text { High-speed data acquisition / fault channels, }>25 \\
\mathrm{MSa} / \mathrm{s}\end{array}$ & $\geq 32$ \\
\hline Interlocks & $\begin{array}{l}\text { Consistent with current HVCM systems, } \\
\text { available upon request }\end{array}$ \\
\hline Local human machine interface & Keyboard/mouse and/or touchscreen \\
\hline Remote human machine interface & EPICS via Ethernet \\
\hline Controller power & $208 \mathrm{~V} \mathrm{rms,} 3$ phase, uninterruptible power supply \\
\hline Reliability MTBF & 24,000 hours \\
\hline Mean time to repair, $90 \%$ of failures & $\leq 2$ hours \\
\hline Minimum component voltage rating & $150 \%$ of maximum operating level \\
\hline Operating temperature & $50-90^{\circ} \mathrm{F}$ \\
\hline Cooling water & $\begin{array}{l}\text { Deionized }(1-5 \mathrm{M} \Omega \cdot \mathrm{cm}), \leq 60 \mathrm{gpm}, \leq 90^{\circ} \mathrm{F} \text { supply } \\
\text { temperature }(20 \mathrm{gpm} \text { for SCR controller })\end{array}$ \\
\hline Maximum size transformer & 170 in. $\times 110$ in. $\times 110$ in. tall \\
\hline Maximum weight transformer & $15,000 \mathrm{lb}$ \\
\hline Maximum size SCR controller & 50 in. $\times 50$ in. $\times 120$ in. tall \\
\hline Maximum size modulator & $170 \times 100 \times 90$ in \\
\hline Maximum weight modulator tank, no oil & $4,500 \mathrm{lb}$ \\
\hline Modulator dielectric fluid & Envirotemp FR3 \\
\hline
\end{tabular}

\subsubsection{Existing Normal Conducting Linac Cavities}

The additional beam current required for the PPU project does impact the warm section modulators. Most of the warm linac structures should be capable of being operated at higher beam current with sufficient RF control margin, assuming minimal modulator changes, once the new controllers are installed (July 2017) and pulse flattening is implemented. The new controllers, in addition to adding enhanced data 
acquisition and troubleshooting features to the modulators, provide frequency and phase shift modulation capabilities to the IGBT switches. These capabilities allow for compensation of the capacitor bank voltage droop and have been shown to be capable of reducing the output cathode voltage droop to less than $0.5 \%$ on all current modulator configurations [10]. Current plans are for the new controller installation to be complete at the time of PPU commissioning. These upgrades are presently being implemented as operational improvements independent of PPU.

\subsubsection{DTL Modulator Systems}

Most of the DTL-section klystrons can be driven with the current HVCM systems. The increased beam load will require additional forward RF power compared with the current operating levels. The HVCM that drives the RFQ and the first two DTL klystrons currently operates with a DC bus voltage of $\pm 1120 \mathrm{~V}$. It will require the highest DC bus voltage change to support the PPU-an increase to $\pm 1200 \mathrm{~V}$. Although the reliability of the IGBTs is severely impacted by the DC bus operating voltage, the recent addition of snubber circuits alleviates this concern [11]. Reliable operation at higher DC bus voltages has been demonstrated with test stand operation of the IGBTs at up to $\pm 1250 \mathrm{~V}$ for several hundreds of hours. It will be further tested in FY 2017 on the RF Test Facility (RFTF) HVCM test stand (although only on a pair of $2.5 \mathrm{MW}$ klystrons) to verify reliable operation is achievable at these increased voltage levels.

In the DTL section of the accelerator, it is necessary to replace the existing $2.5 \mathrm{MW}$ klystrons with 3.0 MW klystrons in the DTL-4 and DTL-5 klystron locations to maintain the desired 25\% LLRF control margin. However, to do so without any other changes would require operation of the IGBTs at voltage and current levels that would hamper reliability. Based on preliminary data from CPI (the manufacturer), simulations were performed to investigate what changes could be made to the existing HVCM topology to reliably deliver the required pulses to the klystrons. By increasing the boost transformer turns ratios and reducing the transformer leakage inductance to approximately $4 \mu \mathrm{H}$, the desired output pulses can be achieved. We are working with Stangenes Industries to determine the feasibility of producing these transformers, as it does offer a challenge to achieve the low leakage inductance while still maintaining adequate clearances to hold off the high voltage. Another solution, although not as attractive, is to use a slightly higher leakage inductance combined with replacing the resonant capacitors.

Another implication of powering the new 3.0 MW DTL-4 and DTL-5 klystrons by a single modulator is reconfiguration of the RF stations. Currently, one modulator powers the DTL-3 and DTL-4 klystrons and another powers DTL-5 and DTL-6. This is a fairly minor reconfiguration, but it has implications for RF, controls, timing, and possibly other parameters. The added cable length could also result in higher available fault energy in the event of a klystron vacuum arc, although the $2.5 \mathrm{MW}$ klystron has been tested with 200 feet of cable in the RFTF and found to survive a vacuum arc.

\subsubsection{CCL Modulator Systems}

Minor modulator changes in the CCL section may be required, depending on how much control margin is required once pulse flattening is implemented. Changes would be necessary only if the DC bus voltage were to exceed $\pm 1200 \mathrm{~V}$. Simulations indicate that, to achieve the required PPU beam current, the DC bus voltage must be close to that level. Should the DC bus level be higher than $1200 \mathrm{~V}$, changing the boost transformer turns ratio to increase the output voltage and implementing thermal upgrades on affected components should achieve the desired results. These changes can easily be tested on the RFTF HVCM system. 


\subsubsection{Technology Readiness}

As discussed earlier, several activities are necessary to demonstrate technology readiness for the warm linac HVCM systems. The simplest activities involve testing the existing DTL and CCL solutions that do not require HVCM modifications on the RFTF test stand. This work will require approximately 3 months of operation time to fully demonstrate that reliable operation can be achieved over 30 days for each of the two RF stations. These tests are unlikely to begin until after the new RFQ is removed from the BTF, but if a second modulator for the BTF cannot be identified, it may be difficult to find available time for PPU testing on the RFTF test stand.

The other activities focus on determining the required modifications and assessing the impact on system reliability of powering two 3.0 MW klystrons. Since no klystrons currently exist at ORNL that have the correct perveance parameters and long lead times likely to be associated with the delivery of these klystrons, another solution must be found. We are communicating with industry representatives to determine if a custom resistive load can be purchased to mimic a pair of klystrons and operate at full average power levels. Alternatively, we are investigating the design of a salt solution load that could be designed in-house and fabricated locally. It would provide us the opportunity to test this HVCM without the need for a klystron load.

Additionally, changes are required to the HVCM components to ensure adequate reliability and to ensure the IGBTs are operating in their safe operating area. We have already performed PSpice simulations to determine the feasibility, assess the required changes, and determine the component parameters. We are also communicating with manufacturers to determine the feasibility of manufacturing these items. However, to perform low-average-power/full-peak-power and full-average-power tests of this modulator configuration with the resistive load will require an additional 4-6 months of testing time on the RFTF modulator.

\subsubsection{Acquisition Strategy}

Where possible, all required component upgrades and/or replacements will be purchased as custom assemblies based on an ORNL-generated specification. When the components vary from those currently employed in the HVCM systems now in operation, sufficient spares will also be purchased at the time of procurement to reduce the overall project cost. Quality will be ensured by requiring the manufacturers to test components to at least $150 \%$ of the operating voltage or the components' voltage ratings, whichever is greater. Additionally, the manufacturers shall be required to test components to relevant ASTM and/or IEEE standards, such as IEEE Std. 390-1987, IEEE Standard for Pulse Transformers.

\subsubsection{New and Existing SCL Cavities}

To provide $805 \mathrm{MHz}$ RF power to the additional 28 cavities required for the STS, twenty-eight $700 \mathrm{~kW}$ peak power klystrons will be installed in the existing klystron gallery. As is typically the case, the ratio of klystrons to modulators is based on optimization of the modulator performance parameters. Because of the increased beam current, additional forward power will need to be supplied to the existing 81 superconducting cavities. This will require additional voltage and current from the modulators.

The required forward power required for each of the additional 28 cavities decreases linearly from $547 \mathrm{~kW}$ to $518 \mathrm{~kW}$ as the beam travels down the linac. This decrease will be used advantageously in the modulators. The first two modulators, each of which powers nine klystrons, generate up to $82 \mathrm{kV}$. The last modulator is then able to power 10 klystrons, rounding out the 28 klystrons, since it can do so by supplying a lower voltage of approximately $80 \mathrm{kV}$. Power and other performance parameters will be virtually identical for the three new modulator systems, with an approximate $15 \%$ power increase in the new modulators compared with the existing ones. 


\subsubsection{Existing SCL Modulator Systems}

The baseline option is to use the existing HVCM topology and increase the voltage to accommodate the higher required output voltage. Figure 4.13 summarizes the issues associated with employing the current HVCM design to power the existing SCL cavities for the PPU based on PSpice simulations. The vertical axis is the output voltage produced from the DC cap bank voltage shown on the horizontal axis. The family of curves represent from 8 to 11 klystrons per modulator. The vertical bar, at approximately $\pm 1220 \mathrm{~V}$ cap bank voltage, represents the $75 \%$ derating of the IGBT maximum collector-to-emitter voltage, below which reliable operation with snubbers should result. The individual dots are the operating levels for the eight existing SCL HVCMs, based on the required forward RF power and the desired $25 \%$ LLRF control margin. All lie on the 10 klystrons per modulator curve, with the exception of SCL-Mod1, which powers 11 klystrons.

As can be seen from Figure 4.13, all existing modulators can operate at or below the $\pm 1220 \mathrm{~V}$ level except for SCL-Mod18. On SCL-Mod18, only two or three of the klystrons require $>77 \mathrm{kV}$. It is likely that these klystrons could be replaced with klystrons with a slightly higher perveance, or the power in those cavities could be reduced slightly to support utilization of the existing HVCM systems. We will perform additional reliability tests on the ten-klystron configuration operating at $\pm 1250 \mathrm{~V}$ in the future to better characterize the mean time between failures (MTBF) of modulators under these operating conditions. This test is easily supported with the existing HEBT Service Building HVCM test stand.

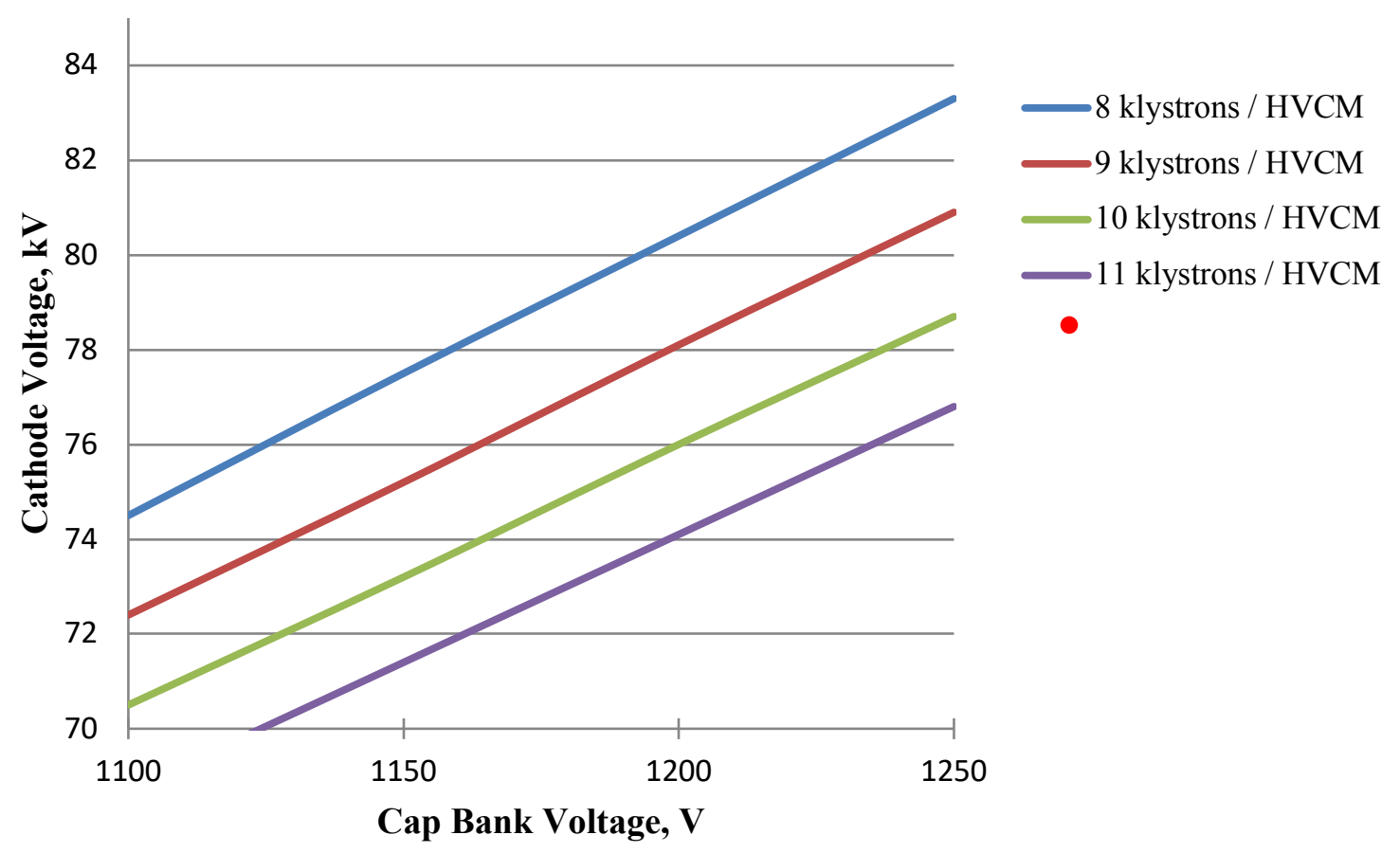

Figure 4.13. Operating voltage levels for existing SCL HVCMs to support PPU forward RF power requirements. Curves shown represent simulation results of modulator output voltage for a given capacitor voltage for different ratios of klystrons to modulators. The vertical line represents the anticipated cap bank voltage limitation that can produce comparable reliability to current operating scenarios when snubbers are installed. Dots indicate operating levels for existing SCL HVCMs. 


\subsubsection{New SCL Modulator Systems}

Unlike the present HVCM, the three phases in the alternate topology modulator (ATM) are series connected (see Figure 4.14 ), and each generates $1 / 3$ of the output voltage ( $\sim 30 \mathrm{kV}$ per phase). A low transformer turns ratio of 7:1 is sufficient to step the $2200 \mathrm{Vdc}$ primary bus, provided by the SCR controller, to $15 \mathrm{kV}$. The voltage is doubled by the transformer's secondary rectifier circuit.

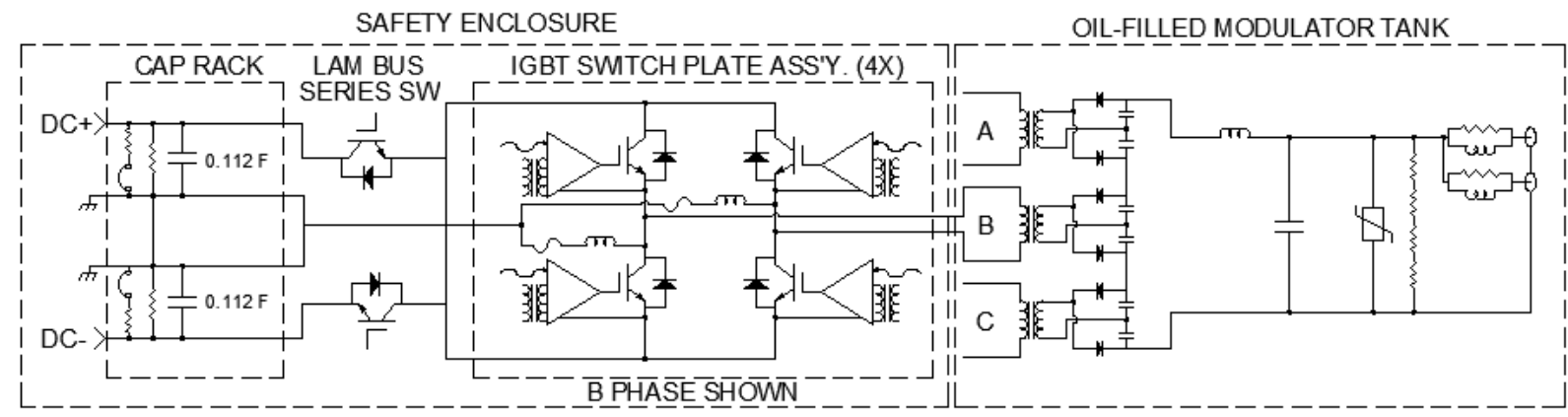

Figure 4.14. Block diagram of ATM topology (showing only a single phase). The series switch shown is not intended to be installed in this installation unless a fourth redundant phase is required.

To switch the IGBT modules used in the H-bridge converters efficiently at high frequency, a resonant circuit is created between the transformer's leakage inductance and circuit capacitance. Here the resonant capacitor across the secondary winding is eliminated, and the output filter capacitors become the resonant components. If the resonant frequency is designed to match the $20 \mathrm{kHz}$ switching frequency, each IGBT will turn on and off under zero current and zero voltage conditions. A PSpice circuit simulation shows the resultant IGBT current and voltage waveforms in Figure 4.15.

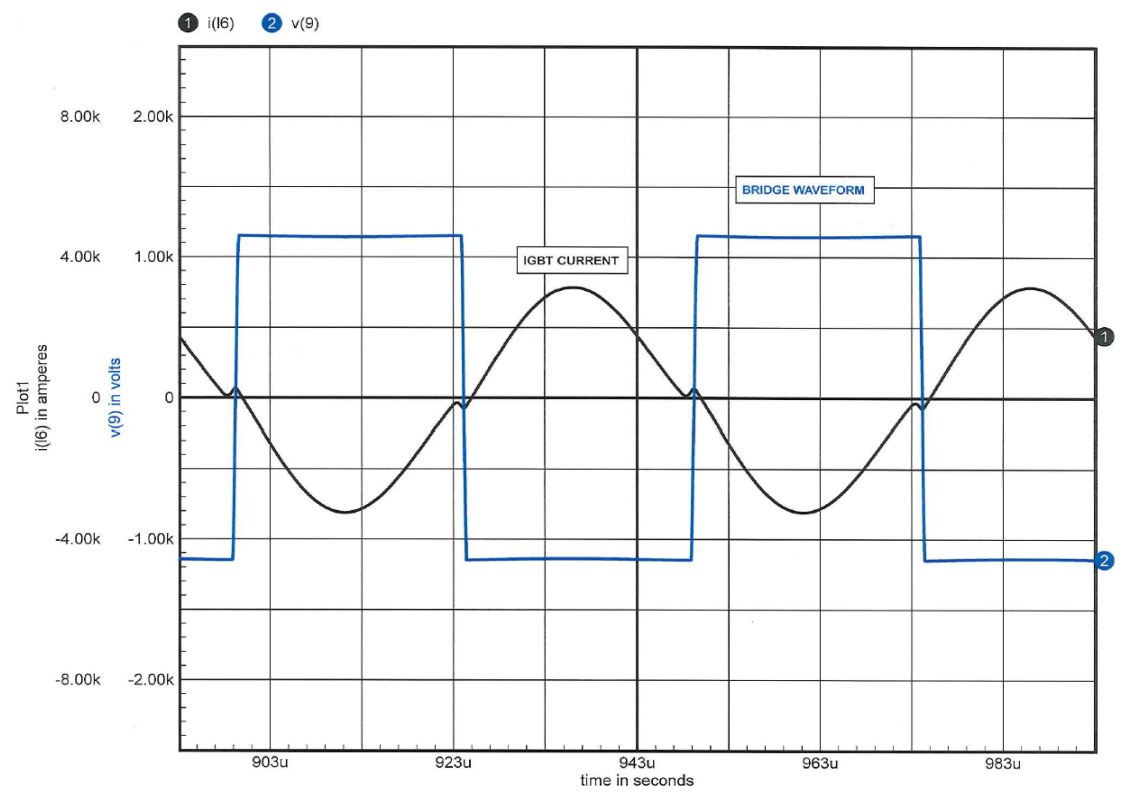

Figure 4.15. PSpice simulation of the ATM IGBT operating waveforms. The black trace is IGBT current. The blue trace is primary voltage across the boost transformer. 
This topology change offers several advantages over the current topology:

- The current HVCM resonant capacitor accounts for a significant amount of modulator downtime. Relocating the resonant capacitor after the rectifier reduces the voltage across the capacitor and eliminates capacitor voltage reversal at every $20 \mathrm{kHz}$ switching cycle. Both of these effects result in improved lifetime compared with the present film/foil capacitors installed in HVCM systems. The lifetime of plastic capacitors is dramatically affected by voltage reversal and scales as the ratio of operating voltage to design voltage to the -8 th power. Implementing the proposed topology permits a longer MTBF design that can be accommodated in the available space.

- Zero voltage switching and zero current switching reduce the IGBT switching losses significantly. This in turn reduces the transient thermal heating of the junction. Since IGBT losses are dominated by switching losses, and every $\sim 10^{\circ} \mathrm{C}$ of operating temperature reduction results in a doubling of the lifetime, this should significantly increase the MTBF of the IGBTs.

- The lower DC bus operating point for the design improves the voltage safety margin for the IGBTs, resulting in additional MTBF gains for the IGBTs.

- Since the transformer turns ratio is reduced from 13:1 to 7:1, it is much easier to achieve the required low leakage inductance values while still maintaining sufficient voltage margin.

- The topology is capable of supporting a wide dynamic load range, driving from 2-10 klystrons with no performance degradation.

Table 4.8 compares operating parameters of the current HVCM system to those of the proposed ATM design. Performance parameters that generally affect the overall lifetime of the modulator are emphasized.

\begin{tabular}{|c|c|c|}
\hline & Current HVCM & Proposed ATM \\
\hline $\mathrm{V}_{\text {out }}$ & $82.6 \mathrm{kV}$ & $82.8 \mathrm{kV}$ \\
\hline Iout & $128 \mathrm{~A}$ & $129 \mathrm{~A}$ \\
\hline Bus $V_{d c}$ & $2.8 \mathrm{kV}$ & $2.2 \mathrm{kV}$ \\
\hline Bus $I_{d c}$ & $310 \mathrm{~A}$ & $390 \mathrm{~A}$ \\
\hline $\mathrm{I}(\mathrm{IGBT})_{\mathrm{pk}}$ & $2.1 \mathrm{kA}$ & $3.1 \mathrm{kA}$ \\
\hline $\mathrm{I}(\mathrm{IGBT})_{\mathrm{rms}}$ & $420 \mathrm{~A}$ & $430 \mathrm{~A}$ \\
\hline I(Diode) $)_{\text {rms }}$ & $22 \mathrm{~A}$ & $63 \mathrm{~A}$ \\
\hline $\left.\mathrm{I}\left(\mathrm{C}_{\text {boost }}\right)\right)_{\mathrm{rms}}$ & $13 \mathrm{~A}$ & $51 \mathrm{~A}$ \\
\hline $\mathrm{V}\left(\mathrm{C}_{\text {boost }}\right) \mathrm{pk}-\mathrm{pk}$ & $91 \mathrm{kV}$ & $30 \mathrm{kV}$ \\
\hline $\begin{array}{l}\text { Transformer step-up } \\
\text { ratio }\end{array}$ & $13: 1$ & $7: 1$ \\
\hline
\end{tabular}

Note that the DC bus (or cap bank) voltage for the current HVCM is $2800 \mathrm{~V}$, or $\pm 1400 \mathrm{~V}$, a level that is clearly too high to achieve reliable operation of the IGBTs. Also note that the boost capacitor for the proposed ATM is three times lower in voltage and yet can use the same volume as the current HVCM. This should result in a significantly more robust boost capacitor. 
The tradeoff to all these voltage reductions for the ATM configuration is that the currents are higher in the IGBTs, diodes, and boost capacitors. This is because each phase of the ATM has to deliver full output current to the klystron; whereas in the current HVCM, because the secondaries are connected in parallel, only $1 / 3$ of the output current is delivered by each phase. However, since most of our past and present failure modes are voltage-related, we anticipate higher overall system reliability even at higher component current operating levels.

\subsubsection{SCL Modulator Systems Technology Readiness}

For the existing SCL HVCM systems, readiness is very good. An identical system has been operated at up to $\pm 1250 \mathrm{~V}$ on the DC bus for hundreds of hours, supplying pulses to beam sticks equivalent to ten klystrons with no issues. However, to further assess the reliability, an extended test on this configuration is scheduled for late 2016/early 2017.

To date, significant progress has been made with the ATM prototype [12]. Transformers and boost capacitors to satisfy the requirements for the modulator have been procured and installed in the modulator tank. The modulator has been tested on the HEBT test stand at $80 \mathrm{kV}$ and $118 \mathrm{~A}$ with a $1.35 \mathrm{~ms}$ pulse, operating at $30 \mathrm{~Hz}$ for several days. Preliminary thermal data collection on the components and pulse flattening studies has been performed. During transition to $60 \mathrm{~Hz}$ operation, a failure in the diode array occurred as a result of the higher operating currents. A higher-current SOT227-B dual diode array was constructed and is currently under evaluation (see Figure 4.16).
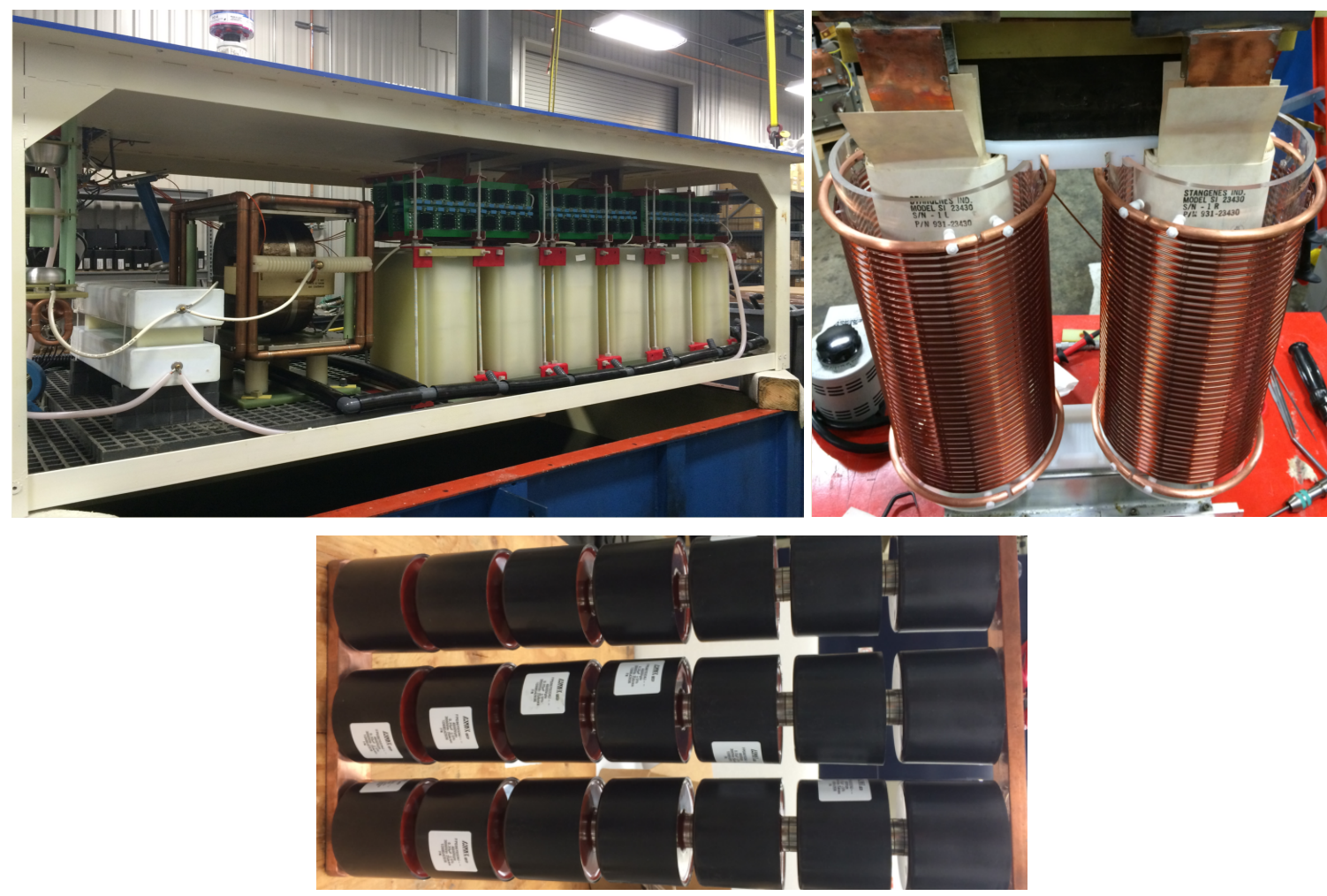

Figure 4.16. ATM tank and other components under development. Upper left: The tank, with highercurrent rectifiers mounted over the six white film/foil capacitors. Upper right: A transformer with circular acrylic secondary windings. Bottom: The array of metallized film capacitors (seven in series, three in parallel).

Additional component development is under way in parallel with the full system testing program. To mitigate corona inception issues, a circular acrylic secondary coil form was fabricated for the boost 
transformer. Additionally, a metallized film capacitor array under development should improve the MTBF of the boost capacitors further and permit "fine tuning" of this important element of the resonant circuit to ensure proper conditions for the IGBTs. These improved components can be seen in Figure 4.16.

Because of obsolescence issues with the IGBTs currently used in all HVCMs, an alternative IGBT will need to be selected for the new SCL systems. An evaluation of suitable devices from four major manufacturers is under way, and a solution should be available by the time the PPU construction project begins. It is possible, as part of this evaluation, to use a device rated for higher current. Doing so would alleviate minor concerns regarding the higher IGBT operating current necessary for the ATM system.

\subsubsection{SCL Modulator Systems Risks and Contingencies}

The risks for the current SCL systems are minimal, as the only real requirement is to increase the operating voltage. Should extended reliability tests uncover issues, a similar approach to what is proposed for the new SCL modulators can be employed. The prototype development activities undertaken to date on the ATM, the current baseline design for the three new modulators for PPU, suggest that the risks are low, although some component issues remain to be resolved. The issues associated with the failure of the original rectifier array have been identified and are addressed with the new array rated for higher current, which is undergoing testing. The HEBT test stand is available to perform additional testing to optimize the new modulator's pulse ripple and droop using frequency and/or pulse width modulation. Once all these issues are addressed satisfactorily, a 30+ day test is scheduled for the second quarter of calendar year 2017.

If the ATM proves to be incapable of meeting the electrical or reliability requirements for the PPU, a modification of the current HVCM system will become the baseline design. By increasing the boost transformer turns ratio and optimizing the boost capacitor value, a design that can achieve parameters that are favorable to reliable IGBT operation is feasible. Based on PSpice simulation, it appears that we can maintain the DC bus voltage at $\pm 1200 \mathrm{~V}$ and keep key component operating current levels comparable to present operating levels.

\subsubsection{SCL Modulator Systems Spares}

Spares are not an issue for the current SCL HVCMs. Sufficient spares are already on hand and are restocked when inventory runs low. Most of the ATM uses identical components to those currently installed in existing HVCM systems. The components that are unique to the ATM-mainly the transformers, transformer bus headers, laminated bus, rectifier assemblies, and boost capacitors - will be stocked at sufficient levels to support continuous operation of the three new SCL modulators. Depending on what is learned about the overall system reliability during extended testing in 2017, it may be desirable to have a complete tank assembly on hand and pretested to serve as a "hot" spare should an in-tank component failure occur during operation.

\subsubsection{Acquisition Strategy for the new ATM SCL Modulators}

The proposed acquisition strategy will be similar to that employed during initial construction of the SNS. Four major procurement packages will be generated for the four major subsystems associated with the modulator: the outside $13.8 \mathrm{kV}: 2100 \mathrm{~V}$ transformer, the SCR controller, the ATM, and the controls electronics. Since the outside transformer and the SCR controller requirements are identical to those currently in use in the other 17 modulators, we will attempt to procure them from a sole source, the same vendor that built the original units. This will minimize the spare parts needed and minimize the amount of additional training of operations staff to operate and maintain these systems. Procurement documentation 
will need to be modified to incorporate some of the upgrades made to these systems over the decade of SNS operation.

The ATM systems will be purchased as a build-to-print subcontract. Since the SNS has a complete set of drawings for the original HVCM, many of which exist as full 3-dimensional native CAD files, it should be straightforward to modify the drawing package to include the components and subassemblies that need to be modified. Documentation associated with the procurement will need to be generated as part of the PPU project.

Controls electronics will be procured and assembled in-house. SNS employees developed the control system and have significant experience in procuring, assembling, testing and installing the system. Since most parts are off-the-shelf or simple custom assemblies, the risk of doing this work in-house is very low.

\subsubsection{Quality Assurance Plan for the new ATM SCL Modulators}

A full fabrication and assembly documentation package will need to be modified and improved to ensure a successful product is delivered. In addition to creating as-built drawings of the system (an effort that is currently under way) industry standards, assembly procedures, component specifications, subassembly and assembly test procedures, acceptance criteria, and other quality documentation will need to be created and incorporated into the procurement package. ISO 9001-certified companies will be used whenever possible.

As experience with the original construction of SNS has revealed, to obtain a quality product, it is imperative to work closely with the vendor manufacturing the system, especially in the case of a build-toprint subcontract. It is anticipated that an engineer will need to spend approximately $30 \%$ of their time at the vendor's facility while assembly and testing are occurring. More time will probably be needed during factory acceptance testing.

Before the award of a subcontract, each company will be evaluated based on an inspection and visit before being considered for the preferred vendors list. Each company's manufacturing capabilities, staff competency, internal quality assurance plans, and infrastructure will be assessed. Companies will be required to perform a full average power test for a minimum of 24 hours whenever possible. Therefore, companies will need to possess infrastructure capable of supporting such a test.

A rigorous subcontract evaluation criteria document will be generated before the release of the request for quotation, and a committee will be selected to evaluate all bids. Weighting of criteria will focus more on technical aspects of the work and less on price to ensure a high-quality product.

\subsection{UTILITIES, NEW COLD LINAC RF COOLING SYSTEM}

A new klystron gallery cooling system (KL-06) shall be provided for the cooling of the RF and modulator equipment that will be added to the open east end of the klystron gallery. Water cooling is required for the RF equipment, themodulators, and existing magnet power supplies at the end of the klystron gallery. The new RF system components that require water cooling include 5 separate TRCC that measure and distribute water to 28 circulators, 28 water loads, and 28 klystrons. The three new HVCMs and three new SCR cabinets will also require water cooling. Finally, the 13 existing magnet power supplies located in the area shall be transferred over to the new cooling system. Figure 4.17 shows the proposed layout for the KL-06 cooling system. Conventional facilities (WBS P.3.9.2) will provide the new enclosure and area utilities. The new cooling system and all associated piping are included within this task.

The design basis for the new KL-06 cooling system is presented in Table 4.9 


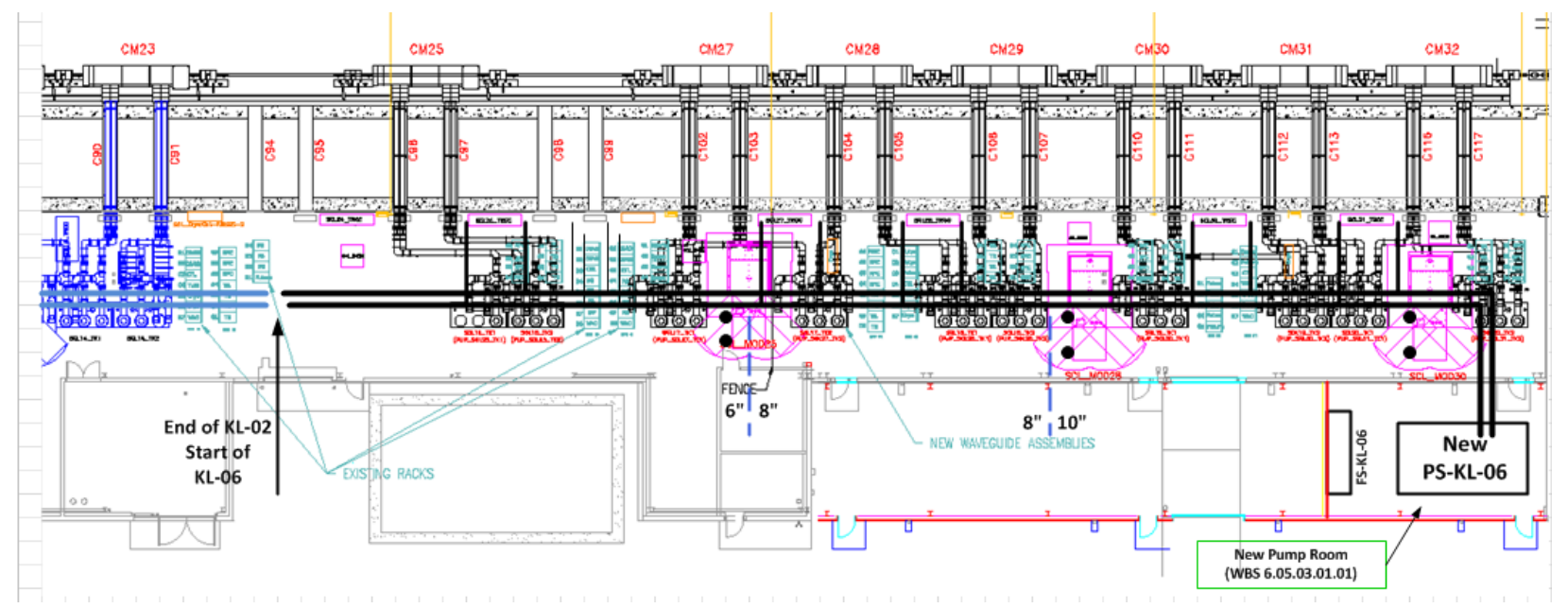

Figure 4.17. Proposed KL-06 RF cooling system layout.

Table 4.9. Design basis for new KL-06 cooling system

\begin{tabular}{|c|c|c|c|c|c|c|c|c|c|}
\hline \multirow{2}{*}{$\begin{array}{l}\text { Technical component } \\
\text { (TC) }\end{array}$} & \multicolumn{3}{|c|}{ HVCM } & \multicolumn{2}{|c|}{ Klystrons } & \multicolumn{2}{|c|}{ RF equipment } & \multirow{2}{*}{$\begin{array}{c}\text { magnet } \\
\text { PS }\end{array}$} & \multirow{2}{*}{$\begin{array}{c}\text { KL-06 } \\
\text { totals }\end{array}$} \\
\hline & IGBT & $\begin{array}{c}\text { Oil heat } \\
\text { exchanger }\end{array}$ & SCR & Coll. & Bodies & Circ. & Loads & & \\
\hline $\begin{array}{l}\text { Required heat removal, } \\
\mathrm{kW} / \mathrm{TC}\end{array}$ & 23.0 & 13.1 & 18.8 & 66.0 & 3.2 & 0.5 & 44.0 & 1.1 & \\
\hline $\begin{array}{l}\text { Required heat removal, } \\
\mathrm{kW} / \text { circuit }\end{array}$ & 23.0 & 13.1 & 18.8 & 198.0 & 3.2 & 1.0 & 132.0 & 1.1 & \\
\hline TC water flow rate, GPM & 30 & 30 & 10 & 50 & 5 & 7 & 15 & 2 & \\
\hline Number of TC & 4 & 4 & 4 & 36 & 36 & 36 & 36 & 13 & \\
\hline Number of TC/circuits & 4 & 4 & 4 & 12 & 36 & 18 & 12 & 13 & \\
\hline Total heat load, kW & 92.0 & 52.4 & 75.0 & $3,376.0$ & 115.2 & 18.0 & 5.7 & 14.3 & 2,749 \\
\hline Total flow, GPM & 120 & 120 & 40 & 600 & 180 & 126 & 180 & 26 & 1,392 \\
\hline Water inlet temp, ${ }^{\circ} \mathrm{F}$ & 92 & 92 & 92 & 92 & 92 & 92 & 92 & 92 & 92 \\
\hline Temperature rise, ${ }^{\circ} \mathrm{F}$ & 5.3 & 3.0 & 12.8 & 27.1 & 4.4 & 1.0 & 0.2 & 3.8 & 13.5 \\
\hline TC press drop, psid/unit & TBD & TBD & TBD & 11 & 15.5 & 38 & 30 & 30 & \\
\hline TC water volume, FT3 & 0.37 & 1.00 & 0.061 & 0.1 & 0.03 & 0.007 & 2.85 & 0.002 & \\
\hline Connection size, inches & 1.5 & 1.5 & 1 & 2 & 1 & 1 & 2 & 0.5 & \\
\hline \multicolumn{10}{|l|}{ Operating pressure, PSIG } \\
\hline $\begin{array}{l}\text { Maximum design pressure } \\
\text { (MAWP), psig }\end{array}$ & 150 & 150 & 150 & 150 & 150 & 150 & 150 & 150 & 150 \\
\hline
\end{tabular}

PSID $=$ pounds per square inch, differential

PSIG $=$ pounds per square inch, gauge

MAWP $=$ maximum allowable working pressure

During normal operation the loads absorbs only $160 \mathrm{~W} /$ load. 


\subsubsection{Upgrade of Existing SCL—RF Cooling Systems}

The existing SCL klystron cooling systems (KL-02 and KL-03) shall be upgraded to allow for an increase in flow and heat transfer area to accommodate an $\sim 20 \%$ increase in thermal loads on the SCL RF equipment. Upgrade work will include an increase in the pump impeller trim, an increase in the motor horsepower and an associated increase in the electrical wiring size and motor starter capacity. Heat exchangers will be rebuilt with additional plates added for the needed heat transfer surface area. The temperature control valve will be resized for the added demand.

\subsubsection{Upgrade of Existing DTL RF Cooling System}

The existing DTL klystron cooling system (KL-04) shall be upgraded to allow for an increase in flow and heat transfer area to accomplish a $20 \%$ increase in thermal loads on the DTL RF equipment. Upgrade work will include an increase in the pump impeller trim, an increase in the motor horsepower, and an associated increase in the electrical wiring size and motor starter capacity. Heat exchangers will be rebuilt with additional plates added for the needed heat transfer surface area. The temperature control valve will be resized for the added demand.

If the new FE-01 is not installed, then KL-04 will have to be upgraded with a larger pump, heat exchanger, headers, and associated equipment similar in size and cost to the new KL-06 system described above. If necessary, installation of the upgrades to KL-04 would require an accelerator outage of 6 to 9 months.

\subsubsection{New Front End Building Cooling System}

The current KL-04 cooling systems provides cooling to the Front End Building (FEB) ion source, RFQ, and MEBT equipment, as well as to all the RFQ and DTL klystrons, circulators, HVCMs, and SCR equipment in the klystron gallery. The $\sim 20 \%$ increase in load on the RFQ and DTL klystrons will require about $125 \mathrm{gpm}$ additional cooling water to meet the additional STS demands. Currently, the front end equipment requires a flow of $110 \mathrm{gpm}$, causing the existing KL-04 pumps to operate above their design point. Operating the pumps outside the design hinders the ability of KL-04 to maintain the desired system pressure in the klystron gallery. To avoid a total removal and replacement of KL-04, we propose placing the FEB equipment on its own separate cooling system (FE-01) and then using the available excess capacity from the FEB for the added STS loads on the RF equipment in the klystron gallery. KL-04 would then be decoupled from the FEB.

The design basis for the new FE-01 cooling system is presented in Table 4.10

Table 4.10. Design basis for new FE-01 cooling system

\begin{tabular}{|c|c|c|c|c|}
\hline Components & $\begin{array}{l}\text { Flow } \\
\text { (gpm) }\end{array}$ & $\begin{array}{l}\text { Diff pressure } \\
\text { (psid) }\end{array}$ & $\begin{array}{l}\text { Heat load } \\
(\mathbf{k W})\end{array}$ & Notes \\
\hline \multicolumn{5}{|c|}{ MEBT } \\
\hline Quad magnets & 20 & 72 & 15 & 14 quad-magnets \\
\hline MISC/diagnostics & 5 & 90 & 1.5 & Turbos, diagnostics \\
\hline \multicolumn{5}{|c|}{ RFQ } \\
\hline RFQ magic tee load & 12 & 25 & 80 & \\
\hline RFQ RF couplers & 2 & 80 & 2 & Two couplers in series \\
\hline LEBT/RFQ turbo-pumps & 15 & 40 & 50 & Seven turbomolecular pump cooling \\
\hline
\end{tabular}


Table 4.10. Design basis for new FE-01 cooling system (continued)

\begin{tabular}{|c|c|c|c|c|}
\hline Components & $\begin{array}{l}\text { Flow } \\
\text { (gpm) }\end{array}$ & $\begin{array}{l}\text { Diff pressure } \\
\text { (psid) }\end{array}$ & $\begin{array}{l}\text { Heat load } \\
(\mathrm{kW})\end{array}$ & Notes \\
\hline \multicolumn{5}{|c|}{ Front end ion source } \\
\hline Turbo pumps & 3 & 45 & 0.42 & $\begin{array}{l}140 \mathrm{~W} \text { nominal for each of three } \\
\text { pumps }\end{array}$ \\
\hline Antenna & 3 & & 5.9 & $\begin{array}{l}80 \mathrm{~kW} @ 7 \% \text { duty factor } \sim 5.6 \mathrm{~kW}+ \\
\sim 300 \mathrm{~W}\end{array}$ \\
\hline Source body cooling & 3 & & 5.9 & Part of above thermal load \\
\hline Matching network & 3 & & 5.9 & Part of above thermal load \\
\hline E-dump & 3 & & 1.3 & Part of other flows \\
\hline MEBT RF power supplies & 5 & 80 & 20 & \\
\hline \multicolumn{5}{|c|}{ Hot test stand } \\
\hline Turbo pumps & 3 & 45 & 0.42 & $\begin{array}{l}140 \mathrm{~W} \text { nominal for each of three } \\
\text { pumps }\end{array}$ \\
\hline Antenna & 3 & & 5.9 & $\begin{array}{l}80 \mathrm{~kW} @ 7 \% \text { duty factor } \sim 5.6 \mathrm{~kW}+ \\
\sim 300 \mathrm{~W}\end{array}$ \\
\hline Source body cooling & 3 & & $"$ & Part of above thermal load \\
\hline Matching network & 3 & & $"$ & Part of above thermal load \\
\hline E-dump & 3 & & 1.3 & Part of other flows \\
\hline \multicolumn{5}{|c|}{ Development stand (new) } \\
\hline Turbo pumps & 3 & 45 & 0.42 & $\begin{array}{l}140 \mathrm{~W} \text { nominal for each of three } \\
\text { pumps }\end{array}$ \\
\hline Antenna & 3 & & 5.9 & $\begin{array}{l}80 \mathrm{~kW} @ 7 \% \text { duty factor } \sim 5.6 \mathrm{~kW}+ \\
\sim 300 \mathrm{~W}\end{array}$ \\
\hline Source body cooling & 3 & & $"$ & Part of above thermal load \\
\hline Matching network & 3 & & $"$ & Part of above thermal load \\
\hline E-dump & 3 & & 1.3 & Part of other flows \\
\hline Future power supply(ies) & 10 & 60 & 10 & Undefined \\
\hline \multicolumn{5}{|c|}{ Near future } \\
\hline Magnetic LEBT & 7 & 60 & 10 & 0.3 gpm per loop/12 loops $/ 2$ magnets \\
\hline $\begin{array}{l}\text { Magnetic LEBT power } \\
\text { supplies }\end{array}$ & 4 & 12 & 24 & $\begin{array}{l}\sim 2 \text { gpm each; } 12 \mathrm{kw} \text { per supply/two } \\
\text { supplies }\end{array}$ \\
\hline Magnetic LEBT diagnostics & 3 & 60 & 0.1 & \\
\hline Total & 128 & & 235.5 & \\
\hline
\end{tabular}

Psid $=$ pounds per square inch differential

\subsubsection{Upgrade of Existing CCL RF Cooling System}

The existing CCL klystron cooling system (KL-01) shall be upgraded to allow for an increase in flow and heat transfer area to accommodate a $20 \%$ increase in thermal loads on the CCL RF equipment. The upgrade work will include an increase in the pump impeller trim, an increase in the motor horsepower, and an associated increase in the electrical wiring size and motor starter capacity. Heat exchangers will be rebuilt, with additional plates added for the needed heat transfer surface area. The temperature control valve will also be resized for the added demand. 


\subsubsection{Electrical Utilities}

\subsubsection{Existing Electrical Utilities and Technical Equipment Cabling}

The existing AC power distribution in the klystron gallery will be the model for the installation of the new equipment needed to support the PPU project. In general, AC power for the existing technical equipment is distributed from exterior pad-mounted switchgear that provides approximately $2000 \mathrm{~A}$ at $480 \mathrm{Vac}$ per unit. The $480 \mathrm{Vac}$ is terminated into multiple distribution or "DP" panels located on the south wall of the klystron gallery. Multiple power panels, designated "PP," and lighting panels, designated "LP," are distributed out of the distribution panels. The power panel distribution is routed through 30-75 kVA transformers that step the voltage down from 480 to 208/120 VAC. The technical loads are then fed from the power panel circuits. In addition, there are some generator and uninterruptible power supply (UPS) -backed power panels, designated "UP," that support critical accelerator loads.

As for the AC power distribution, the cable installation for the technical equipment controls and instrumentation will be modeled on the existing infrastructure. All of the cables necessary for the operation of the five transmitters and seven cryomodules are included in the scope of work and are described in more detail below.

\subsubsection{New PPU Loads}

As mentioned earlier, the new electrical loads for the PPU project include three new HVCMs, five new RF transmitters, seven cryomodules, and other support equipment such as cooling skids and cryomodule controls. Building loads such as heating, ventilation, and air-conditioning systems; utility outlets; and lighting will be installed by $\mathrm{CF}$ electrical personnel. Only technical loads are covered in this report. The conceptual design distributes all AC power from the existing KL-SS4 substation.

Approximate power requirements for the technical equipment are listed in Table 4.11.

Table 4.11. Technical equipment power requirements

\begin{tabular}{lccc}
\hline \multicolumn{1}{c}{ Equipment } & Power (kVA) & Voltage (V) & Comments \\
\hline HVCM & 2700 & 13,800 & Power supply by others \\
HPRF transmitters & 270 & 208 & \\
Cryo-controls & 48 & 120 & \\
LLRF & 115 & 120 & \\
HVCM controls & 7 & 208 & \\
Integrated Control System controls & 10 & 120 & \\
Vacuum & 5 & 120 & \\
Medium-beta SCL cooling upgrade & 250 & 480 & \\
High-beta ACL cooling upgrade & 250 & 480 & \\
Front end cooling system & 30 & 480 & DTL will not be upgraded \\
CCL system cooling upgrade & 250 & 480 & \\
\hline
\end{tabular}

\subsubsection{Electrical Equipment}

The preliminary design includes the installation and use of the power panels listed in Table 4.12. 
Table 4.12. Proposed additional power panels for PPU

\begin{tabular}{lcl}
\hline \multicolumn{1}{c}{ Panel designation } & $\begin{array}{c}\text { Circuit } \\
\text { quantity }\end{array}$ & \multicolumn{1}{c}{ Circuit use } \\
\hline KL-4PP11 & 27 & $\begin{array}{l}\text { Power for cryo-controls, modulator controls, RF controls, HPRF transmitter, } \\
\text { reference line heater }\end{array}$ \\
KL-4PP12 & 32 & $\begin{array}{l}\text { Power for cryo-controls, modulator controls, RF controls, HPRF transmitter } \\
\text { KL-4PP13 }\end{array}$ \\
& 32 & $\begin{array}{l}\text { Power for cryo-controls, LLRF, RF controls, HPRF transmitter, vacuum } \\
\text { system }\end{array}$ \\
KL-4PP14 & 14 & Power for LLRF, HPRF transmitter, reference line heater, vacuum system \\
KL-4PP15 & 25 & Power for LLRF, HPRF transmitter, reference line heater, vacuum system \\
KL-UEP6X & 26 & Uninterruptible power for the vacuum system and cryo-controls \\
\hline
\end{tabular}

The PPU scope includes the installation of four $120 \mathrm{~V}, 20$ A utility receptacles along the base of each new rack row. The klystron gallery CF scope includes the continuation of the main cable tray "ring" in the klystron gallery (see Figure 4.18). The cable tray from the main ring to the racks, tunnel chases, and other technical equipment will be designed and installed as part of the technical utilities scope. In addition, the scope will include any miscellaneous conduit or other raceway necessary to support the PPU cables.

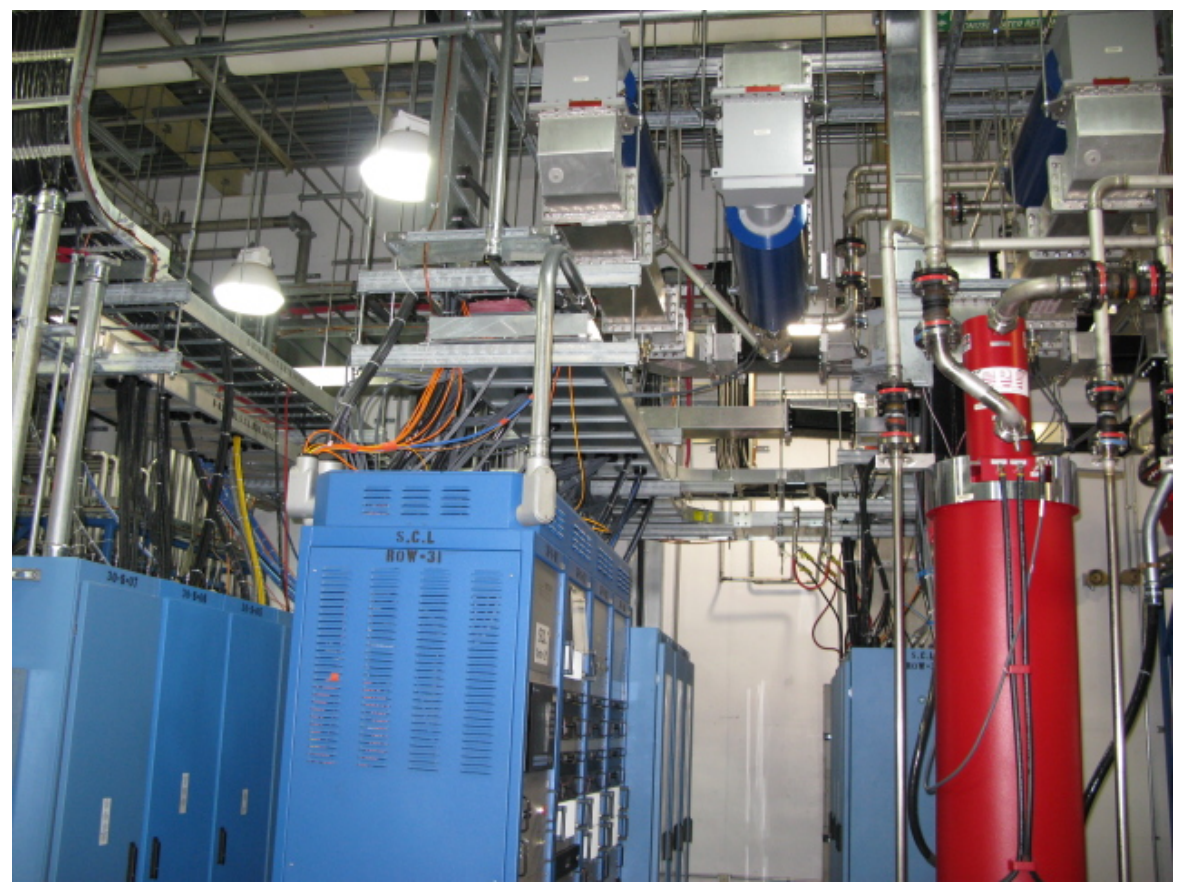

Figure 4.18. Existing cable tray in the klystron gallery.

\subsubsection{Electrical Grounding and Cables}

The PPU project will install the new low-impedance ground plane necessary for the five new RF transmitters and associated three new HVCMs. The design includes approximately $300 \mathrm{ft}$ of $12 \mathrm{in}$. wide copper plane for the main trunk and $250 \mathrm{ft}$ of $8 \mathrm{in}$. wide copper plane for the branches. The copper plane will be riveted flat to the concrete floor, as indicated in Figure 4.19. 


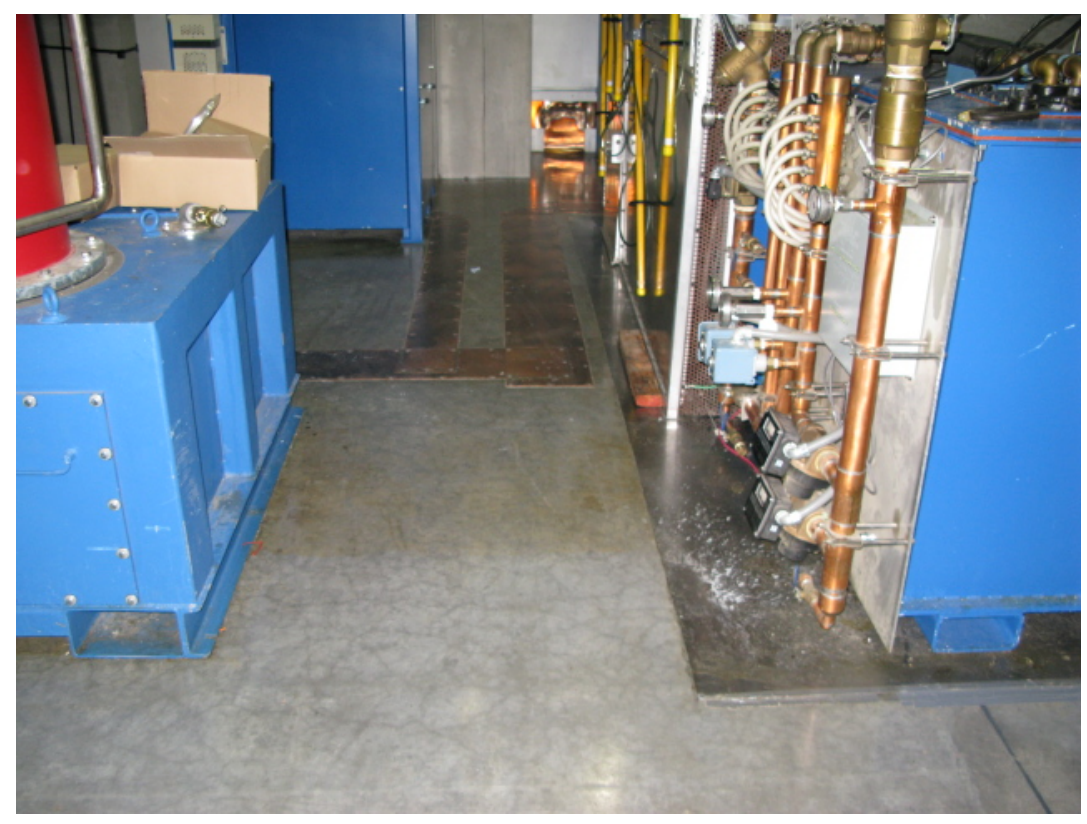

Figure 4.19. Existing ground-plane installation in the klystron gallery.

The PPU project will install bare copper grounding conductors on all raceways, power panels, equipment racks, and other equipment enclosures. The scope of work for the SCL electrical utilities includes the routing and termination of all technical equipment cables. These include cables that route from the gallery into the tunnel. A preliminary, but not exhaustive, list of the cables for each major subsystem is in Table 4.13.

Table 4.13. Preliminary description of cable needs for PPU electrical systems

\begin{tabular}{|c|c|c|}
\hline System name & Cable type & Description \\
\hline \multirow[t]{9}{*}{ HVCM } & $\begin{array}{l}\text { LoxArmor, 3-conductor, } \\
500 \text { kcmils }\end{array}$ & $\begin{array}{l}\text { Armored power cable from outdoor switchgear to the SCR } \\
\text { cabinet }\end{array}$ \\
\hline & Single conductor $4 / 0$ & From the SCR cabinet to the modulator \\
\hline & $\begin{array}{l}\text { Multi-conductor \#10, \#12 and } \\
\# 14 \text { AWG }\end{array}$ & $\begin{array}{l}\text { From the modulator controls cabinet to the switchgear, HVCM } \\
\text { and SCR cabinet }\end{array}$ \\
\hline & Paired cable, \#18AWG & From the HVCM to the $\mathrm{CO} 2$ system \\
\hline & $\# 10, \# 12$ and $\# 14$ power cables & $\begin{array}{l}\text { From the HVCM to C) } 2 \text { system, SCR cabinet and HVCM for } \\
\text { control, ancillary and pump power }\end{array}$ \\
\hline & Coaxial and triaxial cable & Diagnostic and signal cables from HVCM to controls cabinet \\
\hline & Fiber optic & $\begin{array}{l}\text { From HVCM to controls cabinet, from HPRF transmitters to } \\
\text { HVCM, from Timing Master to HVCM }\end{array}$ \\
\hline & Dielectric Sciences 2041-TVJ & High voltage cable from HVCM to HPRF transmitters \\
\hline & Ethernet cable & From HVCM controls cabinet to Accelerator Network \\
\hline \multirow{7}{*}{$\begin{array}{l}\text { HPRF } \\
\text { Transmitters }\end{array}$} & $3 / 8$ in. heliax & From transmitter rack to RF coupler outputs and LLRF racks \\
\hline & High-voltage (HV) coaxial & From transmitter rack to klystron ion pumps \\
\hline & $\begin{array}{l}\text { Multi-conductor \#2 and \#12 } \\
\text { AWG }\end{array}$ & From transmitter rack to the klystron $\mathrm{HV}$ tank EMI enclosure \\
\hline & Multi-conductor \#2 AWG & From transmitter rack to the klystron magnet \\
\hline & Vendor cable & From the transmitter rack to the waveguide circulator \\
\hline & Fiber optic & $\begin{array}{l}\text { From the LLRF racks to arc detector ports and HV tank EMI } \\
\text { enclosure }\end{array}$ \\
\hline & Fiber optic & $\begin{array}{l}\text { From the LLRF racks to arc detector ports and HV tank EMI } \\
\text { enclosure }\end{array}$ \\
\hline
\end{tabular}


Table 4.13. Preliminary description of cable needs for PPU electrical systems (continued)

\begin{tabular}{|c|c|c|}
\hline System name & Cable type & Description \\
\hline \multirow[t]{2}{*}{ LLRF } & $3 / 8$ in. heliax & $\begin{array}{l}\text { From LLRF racks to the SSA input, RF coupler outputs, HOM } \\
\text { couplers, FCM field probes, high-power protection module, } \\
\text { field probes reference line, and local oscillators }\end{array}$ \\
\hline & Fiber optical & From LLRF racks to the window arc detectors \\
\hline & Twin-axial cable & For event utility links \\
\hline \multirow{3}{*}{ System controls } & RS-232 & For SCL controls to cryo-controls \\
\hline & Belden 9512 & For fast protection links \\
\hline & Belden 9512 & For fast-protection links \\
\hline $\begin{array}{l}\text { Personnel } \\
\text { Protection System } \\
\text { (PPS) }\end{array}$ & Multi-conductor \#16 AWG & For HVCM and HPRF PPS connections \\
\hline \multicolumn{3}{|l|}{ Vacuum } \\
\hline & Manhattan M1126 & For vacuum gauge connections \\
\hline & Belden 8219 & For vacuum valves \\
\hline & Belden 9318 & For vacuum controls \\
\hline & Belden 9512 & For vacuum controls \\
\hline & Belden 9344 & For vacuum controls \\
\hline \multicolumn{3}{|l|}{ Cryogenic Controls } \\
\hline & 1-pair \#16 AWG & For the tuner cooling flow switches \\
\hline & 2-pair \#20 AWG & For the liquid helium level probes \\
\hline & 4-pair \#16 AWG & For the cavity and window heaters and signal trunk cables \\
\hline & 4-pair \#22 AWG & For the JT valve LVDT connections \\
\hline & 16-pair \#20 AWG & For the silicone diodes \\
\hline & 8-pair \#20 AWG & For the silicone diodes and thermocouples \\
\hline & 4-pair \#22 AWG & For signal trunk \\
\hline & Coaxial cable & For ControlNet \\
\hline \multicolumn{3}{|l|}{ Cooling Systems } \\
\hline & Commscope 5070 & For PLC (programmable logic controller) communication \\
\hline & Belden 1032A & For instrumentation to PLC I/O \\
\hline & Belden 9368 & For instrumentation to PLC I/O \\
\hline & Belden 27110A & For PLC AC power \\
\hline
\end{tabular}

\subsubsection{RF Waveguide Chases}

Twelve new chase assemblies will be installed to route all cabling and RF waveguides from the klystron galley to the linac tunnel. The chase assemblies were modified slightly from the original design for ease of installation; conduit designations remain the same as the original design. Figure 4.20 shows the 12 penetrations that will receive new chase assemblies (shown in gray). Cryomodules 24 and 26 are not being installed, so no chase assemblies are required in those penetrations $(94,95,98$, and 99), the chase $102 \mathrm{~s}$ assembly is already in place, and the first article of chase number 105 has been installed (summer 2015).

The 12 new chase inserts will be installed before the RF equipment is installed in the klystron gallery and will permit existing chase wiring to be re-pulled into the new chase conduits. 


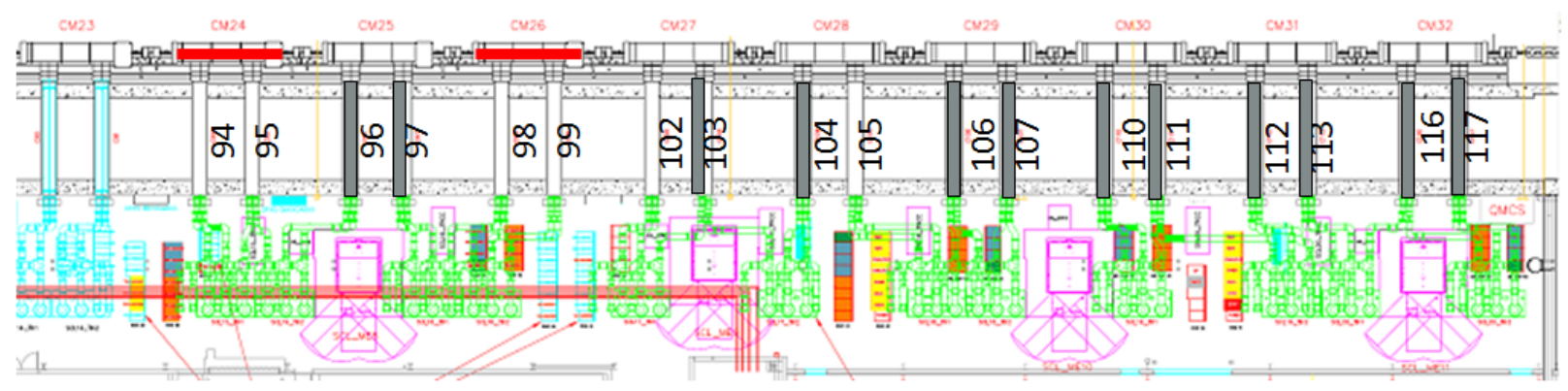

Figure 4.20. Chase assembly penetrations.

\subsection{RF CONTROLS}

Providing controls for the linac RF includes the following:

- EPICS interface to the new HVCMs and supporting IOC hardware.

- Software interfaces to the new RF transmitters. Updates will be required to accommodate the new cryomodule cavity tuner, coupler bias, and coupler cooling control system design. This covers the IOC hardware, programmable logic controller (PLC), ladder logic, and EPICS software development.

- EPICS interface to the new low-level RF systems.

- SCL cavity tuner motor controls will require a new design, as obsolescence prevents duplicating our current controller implementation. A PLC-based commercial-off-the-shelf hardware solution is under consideration.

- SCL coupler bias power supply controls.

- SCL coupler cooling water controls will also require a new design as obsolescence prevents duplicating our current controller implementation. A PLC-based commercial-off-the-shelf hardware solution is under consideration.

\subsection{GLOBAL CONTROLS}

The SNS Integrated Control System (ICS) is a large distributed control system based on the EPICS toolkit. The ICS serves to integrate a diverse array of hardware to allow operators to control the accelerator using a common set of tools. The architecture is scalable, allowing new devices for the PPU to be integrated without changes to the underlying ICS architecture. For the PPU, Global Controls includes four systems: Controls Infrastructure, Timing System, Machine Protection System (MPS), and Personnel Protection System (PPS). These systems will be extended to support the areas where PPU equipment will be installed.

Additionally, controls hardware and software will need to be developed for each new PPU system (e.g., cryomodule, RF, CF, target). In general, these subsystems will be controlled in a similar manner to the corresponding systems already operating at the SNS. These applications typically include commercialoff-the-shelf hardware and application software developed locally. Details for control of these devices are included in the chapter for the corresponding system. 


\subsubsection{Controls Infrastructure}

The controls infrastructure includes the private network, which facilitates moving data among the various computers and servers and workstations that store data and serve files to distributed processors and operator workstations. The ICS network will be extended to accommodate new equipment for the SCL extension. Network specifications for new equipment are the same as for equivalent existing operational equipment. The existing server infrastructure is sufficient to handle the expected additional computational load for the PPU.

Changes and additions to the network will include the following:

- "Edge switches" will be placed in the new rack rows housing PPU accelerator equipment at the east end of the klystron gallery.

- Some existing switches will require replacement to provide additional network ports to accommodate new equipment.

- An "aggregator switch" will be added to communication room 6 in the klystron gallery to accommodate the addition of the edge switches.

No changes to the SNS Central Control Room are anticipated for the PPU. The existing SNS suite of operator interface tools, based on EPICS and Control System Studio, will continue to be used for the PPU. These tools will be configured to include the new components added by the PPU.

\subsubsection{Timing System}

The PPU timing requirements will be handled by extending the existing SNS timing system infrastructure. Timing system equipment required for the PPU includes local timing receiver boards, timing link fan-outs, and distribution panels to extend the system to the newly populated SCL zones. The timing specifications are unchanged for the PPU, and there are no anticipated changes to the timing master, event link, or real time data link. EPICS interface software changes will be required to support the IOCs and timing hardware in the additional zones.

\subsubsection{Machine Protection System}

The MPS, which quickly turns off the beam if a beam loss event occurs, meets the functional requirements for the PPU. However, SNS staff can no longer manufacture the MPS custom hardware boards needed to support the expansion of RF equipment into the additional zones because of obsolescence. Redesign of the obsolete boards will be addressed by MPS AIP-39 starting in FY 2017, so SNS expects to be able to produce the needed board equivalents in time to meet PPU milestones. EPICS interface software changes will be required to support the additional MPS hardware installations.

\subsubsection{Personnel Protection Systems}

Safety systems include credited and defense-in-depth controls with the primary function of mitigating postulated accident scenarios that could otherwise result in harm to people or the environment. Hazards and accident scenarios will be addressed throughout the project, culminating in major revisions to the Proton and Neutron Facility Safety Assessment Documents (FSADs). The revised FSADs will describe the credited controls, including protection systems that form the basis for a new accelerator safety envelope. The FSADs are the primary drivers for safety system performance requirements. 
The PPU project will include modifications to the accelerator PPS to accommodate the energy upgrade as well as to integrate new requirements to support the R2T2 tunnel stub. The new safety systems will be functional duplicates of the existing accelerator PPS.

The primary function of safety systems is mitigation of significant hazards addressed through DOE Order $420.2 \mathrm{C}$, and the associated guidance 420.2A [13,14]. Critical devices are used to prevent beam transport to an area that is not in a safe configuration.

The safety system scope and deliverables include the following:

- System level operation, certification, and maintenance documentation

- Safety-system-specific, system-level networking and interconnection infrastructure

- Hardware/software required to interface the PPS systems to EPICS, e.g., "mail-box" PLC

- Safety-system-specific EPICS screens and archiver functions

\subsubsection{Assumptions}

- Accelerator Safety Order-related activities such as the Final Safety Analysis Document and the Accelerator Safety Envelope (ASE) development are outside the scope of the protection system work section.

- $\quad$ The RTBT2 will be accessible only when RTBT1 is in an access state.

- The FTS target will not be damaged by a minimum of two consecutive pulses or 2 seconds at $2.5 \mathrm{MW}$.

- Existing EPICS IOC(s) will be used for data collection and archiving.

- This work involves only the linac PPS and the new RF systems in the klystron gallery.

\subsubsection{Linac Cryomodule Controls}

Controls for the new PPU cryomodule will closely resemble the existing high-beta cryomodule controls. Changes will be required to accommodate silicon diode connections used in the updated cryomodule design. The major design change for the cryomodule controls is centered around the beam line interface and the wiring.

The PPU cryogenic control system includes equipment racks, PLC hardware, IOC hardware, level meters, pressure sensors, power supplies, and marshaling panels. It does not include valves, temperature sensors, heater elements, liquid helium level probe, connectors and wiring attached to the cryomodule, vacuum gauges, or RF instrumentation and wiring.

The cryogenic control systems will, by default, be powered from the UPS. An automatic transfer switch will be installed in each rack group and marshaling panel to provide a connection to "normal" power to ensure continued operation if the UPS circuit loses power. EPICS interface software changes will be required to support the new IOCs and cryomodule interface PLCs.

Because connectors will be installed before the conduit is pulled through, the connector size and number of conductors in a cable are important. Based on past experience, it was determined that the largest particle size for a connector that could be pulled through the conduit would allow the termination of an 
eight-shielded, twisted-pair cable. This determination changes the number and types of cables to be installed. It also forces the revision of most of the cryomodule control system drawings.

\subsubsection{Known Issues}

The tunnel access time required for cable installation and termination and cryomodule checkout must be greatly reduced from the time required for the original SNS construction. A new approach is proposed for the installation of cryomodule control system tunnel wiring to address this issue.

\subsubsection{Linac Water System Controls}

EPICS and PLC controls will be provided to control the new deionized water system pump loop KL06. The new CF PLC and chassis will be installed in klystron building communication room 6. It will control new pump loop KL06 and new air handler AH-KL-06. Separate I/O panels local to KL06 and AH-KL-6 and the new I/O panels will be connected to the new PLC via Ethernet. EPICS interface software changes will be required to support the new IOC and PLC hardware.

\subsubsection{Beam Line Vacuum Control System}

The beam line vacuum control subsystem provides vacuum controls for the new cryomodules, the warm sections, and the high-energy differential pumping section. These controls are used to monitor and control vacuum components and provide interlock signals to low-level RF systems and the MPS. The existing SCL vacuum controls will be duplicated. Replication of the existing controls, PLC hardware, and ladder logic will be provided to monitor and control the new vacuum components along with the corresponding EPICS interface software.

\subsubsection{Insulating Vacuum System Controls}

The insulating vacuum system controls subsystem provides vacuum controls for pumping the insulating jackets of the cryomodules. The existing insulating vacuum controls for cryomodules 1-16 will be replicated. A typical single SNS insulating vacuum system handles pumping for eight cryomodules. These controls include PLC systems with ladder logic and EPICS interface software.

\subsubsection{Ring Primary and Secondary Stripper Foil System Controls}

EPICS and PLC controls will be provided to accommodate modifications to the primary and secondary stripper foil mechanism. The existing VME controls hardware is no longer available, so a new motor control design will be required. A PLC-based stepper motor controller is under consideration. The existing Ring Service Building racks will house the new equipment.

\subsubsection{Ring Water System Controls}

EPICS and PLC controls will be provided to accommodate modifications to the existing deionized water system (DIWS) RN03 and implementation of the new DIWS RN04. The existing PLC system will be used to provide the processing capabilities with a new remote I/O panel installed in the Ring Service Building basement. 


\subsection{REFERENCES}

[1] M. Lynch, W. Reass, and P. Tallerico, "SNS Linac RF system overview," Proceedings of the 1999 Particle Accelerator Conference, eds. A. Luccio and W. W. MacKay, New York, March 29-April 2, 1999.

[2] M. Champion, "SNS RF system performance and operation," Proceedings of Particle Accelerator Conference (PAC07), ed. C. Petit-Jean-Genaz, Albuquerque, New Mexico, June 25-29, 2007.

[3] T. Hardek, M. Crofford, Y. Kang, et al., "Status of the Oak Ridge Spallation Neutron Source (SNS) RF systems," Particle Accelerator Conference, New York, March 28-April 1, 2011.

[4] MEGA Industries, MEGA Industries RF Solutions, retrieved from Waveguide: http://www.megaind.com/waveguide/, August 3, 2016. Accessed September 2016.

[5] Design Criteria for Linac RF, SNS-104010000-DC001, Oak Ridge National Laboratory, November 30, 2000.

[6] M. Champion et al., "Overview of the Spallation Neutron Source linac low-level RF control system," Proceedings of the 2005 Particle Accelerator Conference (PAC05), Knoxville, Tennessee, May 1620, 2005.

[7] M. Crofford et al., "Spallation Neutron Source LLRF temperature dependence and solution," LINAC10, Tsukuba, Japan, September 2010.

[8] M. Piller et al., "The Spallation Neutron Source RF reference system," Proceedings of the 2005 Particle Accelerator Conference (PAC05), Knoxville, Tennessee, May 16-20, 2005.

[9] W. Reass et al., "High-frequency multimegawatt polyphase resonant power conditioning," IEEE Transactions on Plasma Science, 33(4), 1210-1219 (August 2005).

[10] G. Patel et al., "Experimental results from droop compensation for the high voltage converter modulators," IEEE Transactions on Dielectrics and Electrical Insulation, 20(4), 1093-1100 (August 2013).

[11] D. E. Anderson et al., "Recent developments in the improvement campaign for the high voltage converter modulator at the Spallation Neutron Source," pp. 672-675 in Proceedings of IEEE International Power Modulator and High Voltage Conference, Santa Fe, New Mexico, 2014.

[12] D. Solley et al., "HVCM topology enhancements to support a power upgrade required by a Second Target Station (STS) at SNS," pp. 362-365 in Proceedings of IEEE International Power Modulator and High Voltage Conference, San Diego, June 3-7, 2012.

[13] US Department of Energy, Safety of Accelerator Facilities, DOE O 420.2C, Department of Energy, Washington, DC. 2011.

[14] US Department of Energy, Accelerator Facility Safety Implementation Guide for DOE O 420.2C, Safety of Accelerator Facilities, DOE G 420.2-1A, Department of Energy, Washington, DC, 2014. 



\section{RING SYSTEMS}

The ring will need some minor modifications to the injection and extraction areas. The ring RF system may also need minor alterations to accommodate the higher beam loading effects. All other areas in the ring are already able to support $1.3 \mathrm{GeV}$ beams. The upgrade plan is consistent with that developed for the earlier Power Upgrade Study [1].

The particle tracking simulations and the space charge effects described earlier show the ring should perform as expected at higher beam power levels. The following sections describe the modifications to the ring hardware.

\subsection{INJECTION REGION}

The purpose of the ring injection system is to inject particles from the HEBT into the accumulator ring and transport the waste beams to the ring injection dump. The key components of the injection system are shown in Figure 5.1. Some of the injection chicane magnet currents cannot simply be scaled up to accommodate the increased injection energy of $1.3 \mathrm{GeV}$ because this would cause excessive $\mathrm{H}^{-}$stripping. Two of the four chicane magnets will be replaced with a new design, and the injection dump septum magnet will require a new design.

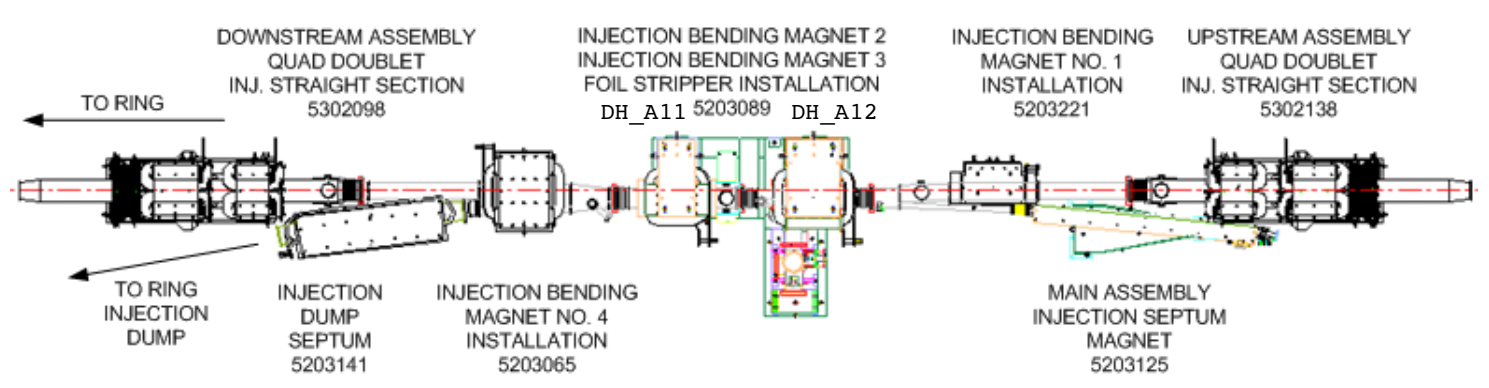

Figure 5.1. Injection system major components.

Chicane magnets. The following modifications are required for the bending (chicane) magnets:

- move magnet 5203221 (DH_A10 Chicane 1) upstream

- redesign and replace magnet 5203072 (DH_A11 Chicane 2)

- redesign and replace magnet 5203104 (DH_A12 Chicane 3)

Both redesigned magnets will retain the basic configuration and mechanical design of the existing units. New vacuum vessels will be necessary to accommodate the new magnets and to allow the stripper foil mechanism to be relocated. The existing power supplies will likely be adequate for the new chicane magnets.

Injection dump septum magnet. The magnet steel in the existing injection dump septum magnet 5203141 will be driven into the highly saturated region to achieve the magnetic fields required for $1.3 \mathrm{GeV}$ operation, exceeding the current available from the present power supply. Therefore, this magnet will be replaced with a new design that will occupy the same approximate installation footprint. Like the chicane magnets, this magnet will retain the basic configuration and mechanical design of the existing unit. The vertical aperture through the magnet will be at least as large as in the present design, and the horizontal aperture will accommodate the waste beams with appropriate clearance on each side. A new vacuum vessel for this magnet is also required. It is likely that the existing power supply will be sufficient for the new septum magnet. 
Injection kicker system. The injection kicker magnets by themselves are capable of operating at the higher currents necessary to deflect the $1.3 \mathrm{GeV}$ beam. Empirical field tests at high duty factors show the thermal performance of the magnets is adequate for the required PPU operational parameters. However, the power supplies for the kicker magnets will require upgrades. All eight injection kicker power supplies will be upgraded from 1400 to 1550 A to provide the extra field required to deliver the same kick angles at the higher $(1.3 \mathrm{GeV})$ beam energy.

Ring stripper foils. To maintain stripping efficiency at $1.3 \mathrm{GeV}$, the stripper foil thickness must be increased. Operational experience at the present $0.97 \mathrm{GeV}$ injection energy of has shown a preference for $0.38 \mathrm{mg} / \mathrm{cm}^{2}$ thick nanocrystalline diamond foils. As illustrated in Figure 5.2, scaling this energy to $1.3 \mathrm{GeV}$ requires a thickness of $0.413 \mathrm{mg} / \mathrm{cm}^{2}$. The thicker foil, combined with the higher beam currents, will increase the foil temperature. Simulations predict the temperature will be about $300 \mathrm{~K}$ higher (personal communication from Y. Takeda, J-PARC, to M. Plum ORNL, April 2013). To ensure the foil will continue to survive, a series of tests is planned. To mimic the heat load expected for the PPU beams, tests will be based on increasing the number of foil hits on the foils installed in the ring. Other tests will use the foil test stand, which comprises a $30 \mathrm{keV}, 5 \mathrm{~mA}$ peak pulsed electron beam designed to produce PPU-level heat loads, although with relatively smaller beam spots.

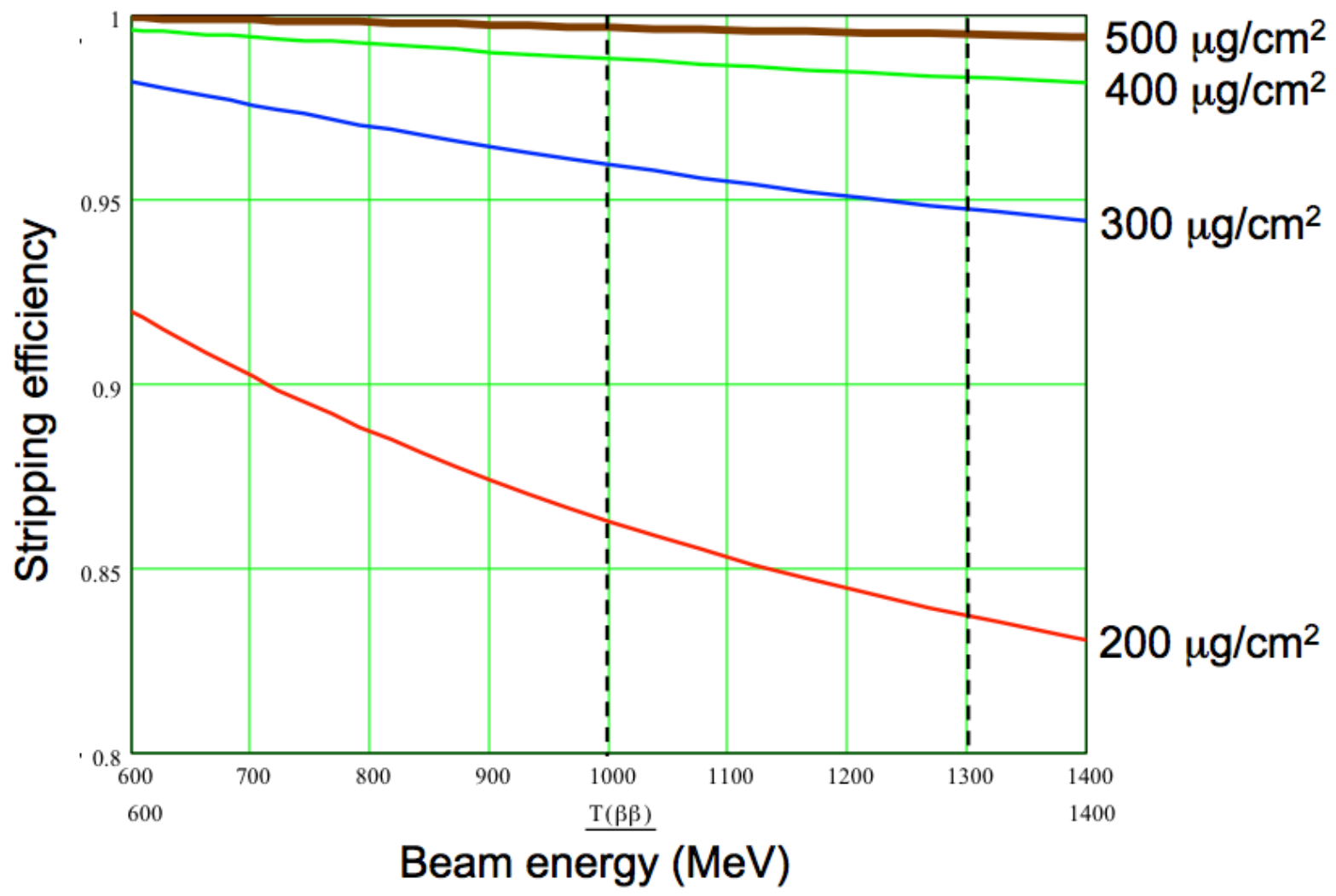

Figure 5.2. Stripping efficiency vs. foil thickness for a $1.3 \mathrm{GeV} \mathrm{H}^{-}$beam.

\subsection{EXTRACTION REGION}

The purpose of the extraction system is to redirect the trajectory of accumulated proton bunches from the ring toward the target via the RTBT. The magnets making up the extraction system are shown in Figure 5.3. 


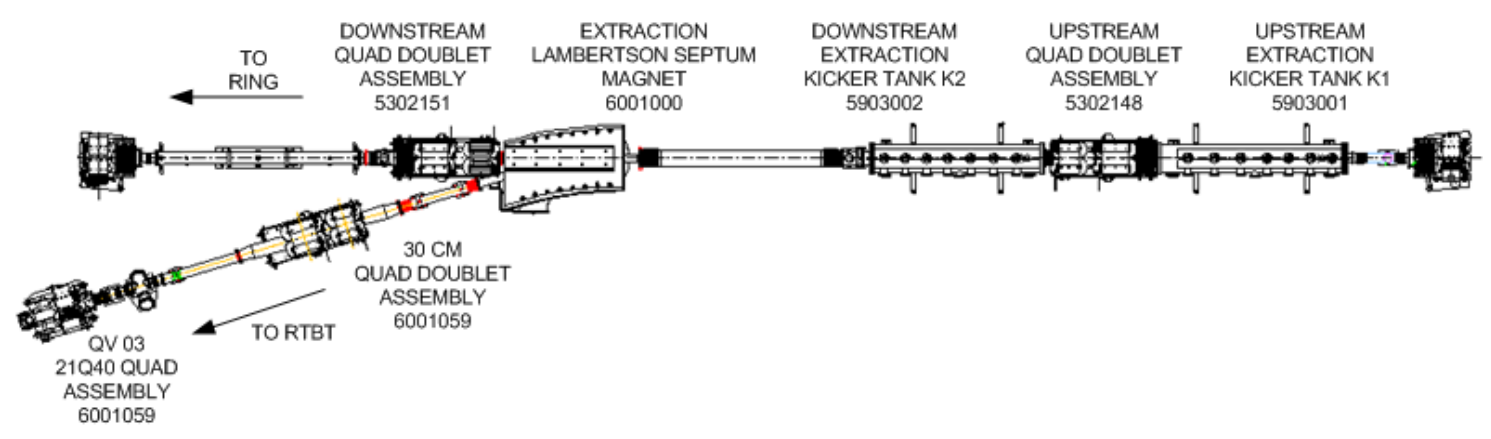

Figure 5.3. Extraction system magnets.

Each extraction kicker vacuum tank contains seven single-turn ferrite magnets. There are six slightly different magnet designs, primarily distinguished by vertical aperture size. The identification and installation arrangements for the existing kicker magnets are shown in Figures 5.4 and 5.5.

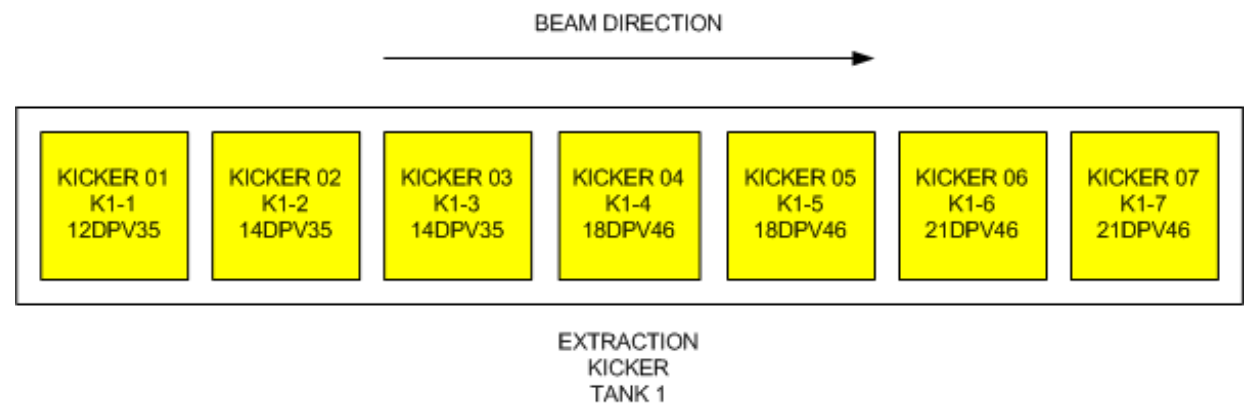

Figure 5.4. Schematic of the existing extraction kicker tank K1 with magnet types.

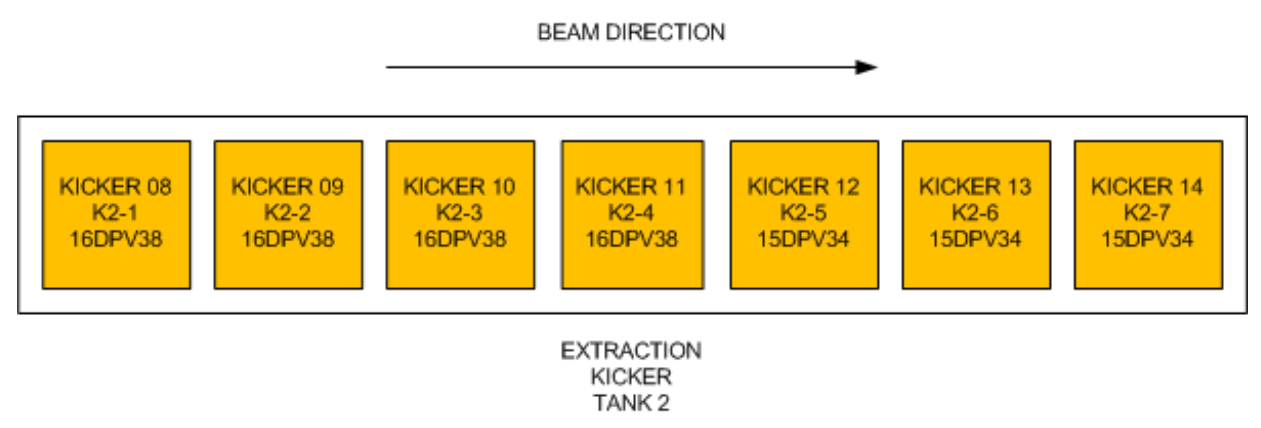

Figure 5.5. Schematic of the present extraction kicker tank K2 with magnet types.

To accommodate the $1.3 \mathrm{GeV}$ PPU beam energy, two more kickers will be added to this system for a total of 16 magnets. A vacuum tank extension, or annex, will be added to the upstream end of K1 and to the downstream end of K2. One additional kicker of the same type as the first and last magnet in the respective main tanks will be housed within each annex. The spacing between the upstream annex magnet and K1-1 must match the spacing between K1-1 and K1-2. Likewise, the spacing between the downstream annex magnet and K2-7 must match the spacing between K2-6 and K2-7. The new magnet arrangement and renumbering is shown schematically in Figures 5.6 and 5.7. 


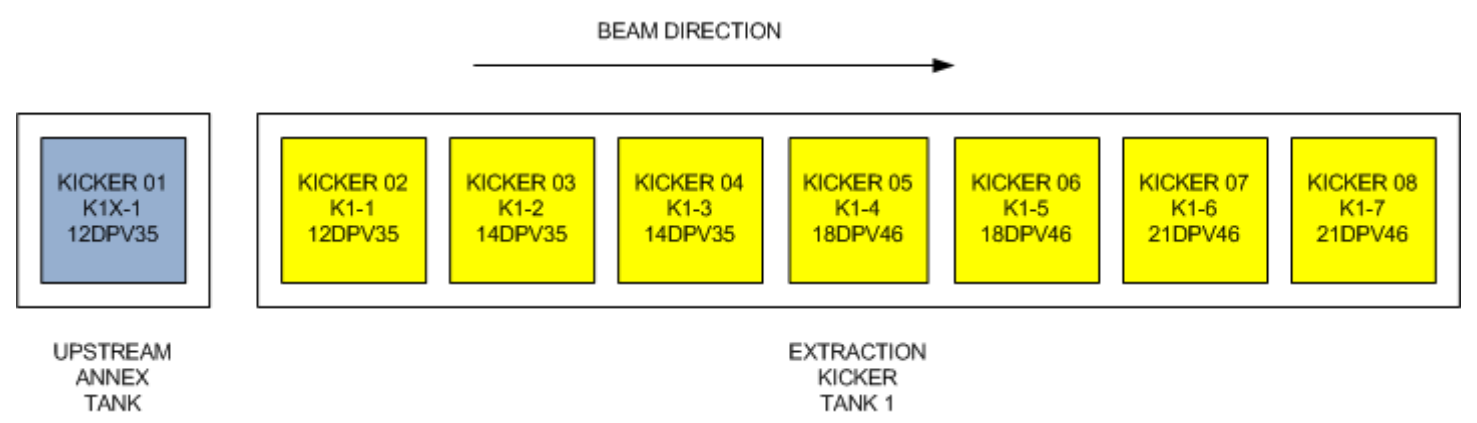

Figure 5.6. Schematic of extraction kicker tank K1 with annex and renumbered magnets.

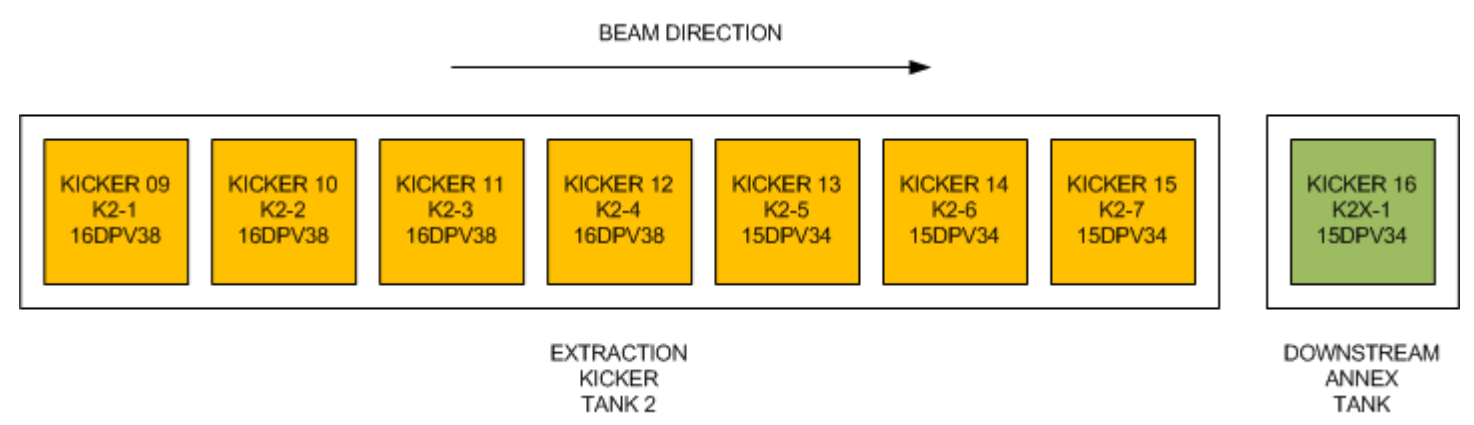

Figure 5.7. Schematic of extraction kicker tank K2 with annex and renumbered magnets.

To summarize, the new extraction kicker magnets system will include the following:

- The upstream annex magnet will be a type 12DPV35.

- The downstream annex magnet will be a type 15DPV34.

- Both magnets will require titanium nitride coating.

- The eddy current strips on the existing magnets within both main tanks will be extended to the new magnets.

- The eddy current strips will be grounded at the upstream and downstream ends of the respective K1 and $\mathrm{K} 2$ annex tanks.

Each of the two additional kicker magnets will require a new pulse forming network (PFN) with the requisite power supply, of the same type presently in use. Also, each of the two new PFNs will require a new oil-cooling skid connected to the heat exchanger of the existing tower water system. New sets of kicker magnet power cables will be pulled for the additional magnets. The required rack space in the Ring Service Building was reserved for this purpose during SNS construction and is still available.

Extraction Lambertson septum magnet. At beam energies higher than $1.0 \mathrm{GeV}$, the power setting of the extraction Lambertson septum magnet, shown in Figure 5.8, will require pole tip shims designed and optimized for $1.3 \mathrm{GeV}$. This is needed to cancel the skew quadrupole component in the magnetic field. 


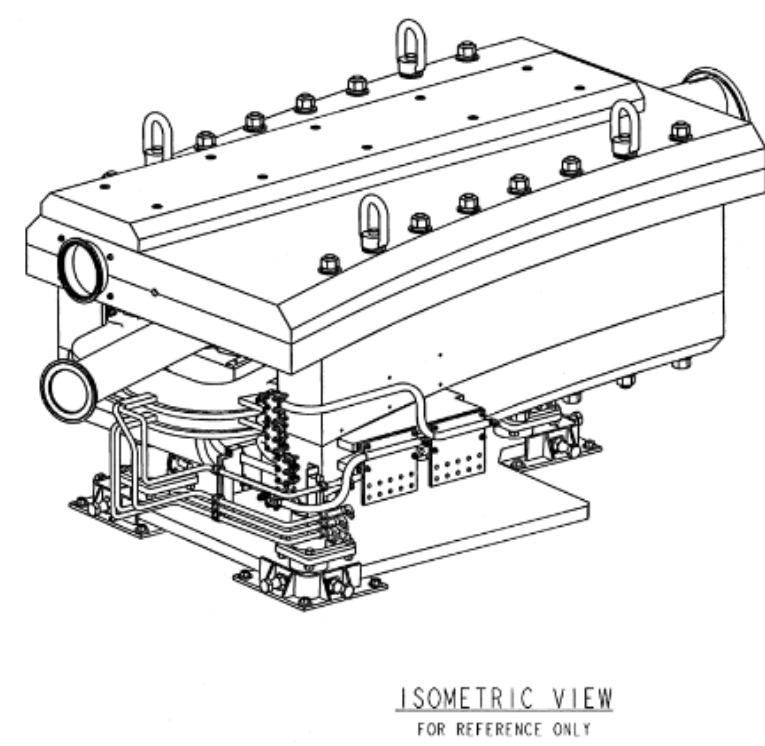

Figure 5.8. Extraction Lambertson septum magnet.

\subsection{RING INJECTION DUMP}

The ring injection dump is presently rated for $1.3 \mathrm{GeV}, 150 \mathrm{~kW}$. This power rating is based on thermodynamic simulations of the water-cooled dump, the concrete that surrounds the dump, and the earth that cools the concrete. During normal operating conditions, about $5 \%$ of the linac beam power is delivered to the injection dump, and so the dump power rating of $150 \mathrm{~kW}$ is well matched to $3 \mathrm{MW}$ linac operation. However, it can occasionally be desirable to operate the accelerator with lower stripping efficiency (e.g., because of short-term stripper foil issues). In these cases, it could be desirable to deliver more than $150 \mathrm{~kW}$ to the dump. A series of thermocouple sensors mounted at various positions around the dump have been periodically read out since initial operation of the SNS. For the PPU project, these data will be analyzed to determine if it is practical to increase the dump power rating.

The injection dump itself, and the proton beam vacuum window in front of the dump, work best when the beam is centered. There are eight thermocouples mounted to the rim of the window to help center the beam. However, these thermocouples provide only a crude measure of the beam position and very little information about the size and distribution of the beam. The PPU project will therefore design, fabricate, and install a beam imaging system that will allow measurement of the beam position, size, and distribution at the entrance to the dump. It will be similar to the beam imaging system currently used on the FTS. This will reduce the risk of compromising the performance of the dump at the PPU and STS beam power levels.

\subsection{RING UTILITIES}

Ring injection magnet cooling water system. Several of the ring injection magnets will be replaced with new magnets. The existing lines will be disconnected and then reconnected to the new magnets once the magnets are installed. Flow and flow requirements for the new magnets will be the same as or similar to those for the original magnets. The existing ring magnet cooling systems (RN-01) have excess flow and heat capacity under current operation (see Section 10.2). The cooling water system will need to be rebalanced once the changes are made.

Ring magnet power supply cooling systems RN-03 and RN-04. As a result of the increase in power required of all the ring magnet power supplies, additional heat load and flow will be required to cool the 
existing or replacement power supplies. Based on the power and thermal tests performed on the ring magnet system, it was determined that the existing Ring Service Building power supply cooling system (RN-03) is not adequate to cool the supplies for the PPU power level requirements (see Section 10.2). Therefore, the existing system will be split into two separate cooling systems (RN-03 and a new RN-04). The existing RN-03 system will be modified to cool only the west side of the Ring Service Building, and the new RN-04 system will tie into the existing east side header and cool the remaining magnet and RF power supplies.

Ring extraction magnet power supply (PFN) cooling system. The two new extraction kicker magnets in the ring will require two new PFN power supplies, oil-cooling skids, and connection to the tower water system for cooling the power supply oil in the Ring Service Building-PFN area. Two new oil-cooling skids will be fabricated and installed beside each of the two new PFN oil tanks. New piping will be installed on each oil tank and connected to the pump skid for recirculating the oil from the tank through the skid's heat exchanger and filters. One-inch tower water connections will be extended from the existing tower water headers to each of the new heat exchangers. The system is designed to circulate 20 gpm of oil through each heat exchanger, and the tower water flow will remove up to $5 \mathrm{~kW}$ of heat at 10 gpm of tower water flow.

Electrical utilities. Field tests of ring magnet operation at $1.3 \mathrm{GeV}$ levels have shown that the main ring dipole magnet power supply will require cooling fans be added to the main ring dipole substations. All the other power supplies have sufficient capacity, and no additional electrical power will be required for the Ring Service Building.

\subsection{BEAM TRANSPORT LINES}

\subsubsection{High Energy Beam Transport Line}

The original design anticipated the $1.3 \mathrm{GeV}$ upgrade, so no hardware modifications will be needed to the HEBT line that transports the beam from the linac to the accumulator ring.

\subsubsection{Ring to Target Beam Transport Line}

The RTBT beam line was designed for $1.3 \mathrm{GeV}$, so no changes are needed to accommodate the higher beam energy.

\subsubsection{RTBT to R2T2 Penetration}

The new R2T2 beam line for the future STS project will start about halfway along the RTBT. A beam tunnel stub will be constructed as part of the PPU project to minimize future interference between the R2T2 tunnel construction and the users of the FTS. Temporary shielding will be stacked in the stub. The future STS project will connect the new beam line tunnel to the stub and install the R2T2 beam line components. Details of the R2T2 stub can be found in the conventional facilities section of this report. No beam line components for the new R2T2 line are included in the PPU.

\subsection{ASSUMPTIONS}

In this chapter we assume that by the time the new injection section vacuum chambers are installed, the existing primary and secondary stripper foil changers will be suitable for use in the PPU. We also assume that the electron catcher, which is currently being redesigned, will be suitable for the PPU. 


\subsection{CRITICAL SPARES}

Three new, one-of-a-kind magnets will be designed, fabricated, and installed in the ring injection section. It is not practical to create spares for the complete magnet assemblies. However, it is prudent for the project to procure spare coil packages for the magnets. The extraction kicker systems are copies of existing systems, so no extra critical spares will be required.

\subsection{REFERENCES}

[1] M. Plum, T. Gorlov, J. Holmes, T. Hunter, T. Roseberry, and J. G. Wang, "Challenges for the SNS ring energy upgrade," Proceedings of the International Particle Accelerator Conference (IPAC12), New Orleans, LA, May 20-25, 2012.

http://accelconf.web.cern.ch/AccelConf/IPAC2012/papers/moppd069.pdf. 



\section{PPU FIRST TARGET STATION SYSTEMS}

The design basis for most of the SNS FTS was $2.0 \mathrm{MW}$ with $1.0 \mathrm{GeV}$ proton pulses delivered at a $60 \mathrm{~Hz}$ pulse repetition rate. Two MW will be available to the FTS with the PPU, and the PPU will increase proton energy to $1.3 \mathrm{GeV}$. Higher proton energy and pulse intensity requires reevaluation of the thermal, structural, and shielding margins of FTS systems as well as changes in radiation damage rates. Affected target systems include the mercury target module, mercury process systems, off-gas systems, reflectors, moderators and their cryogenic systems, reflector vessels, shielding, utilities, and instrument line core vessel inserts.

The original FTS design basis employed simplified bounding assumptions for heating in many systems that are expected to provide some margin when evaluations are performed with more accurate methods for the $1.3 \mathrm{GeV}$ beam. Some system upgrades or modified operating parameters may be necessary for 2.0 MW operation.

The mercury target module was an exception to the original FTS design basis, as it was initially rated for 1.0 MW and later increased to $1.4 \mathrm{MW}$ and $1.0 \mathrm{GeV}$ at $60 \mathrm{~Hz}$. Operational experience has influenced the design of the mercury target vessel. Design changes have been incrementally incorporated and fabrication oversight has increased. Soon, gas injection will be initiated at a low flow rate. The jump to $2.0 \mathrm{MW}$ will require more gas flow and further design improvements. PPU FTS Systems' scope includes the development and deployment of high-flow helium gas injections into the mercury target to provide maximum mitigation of beam pulse-induced cavitation erosion and mercury vessel fatigue stress. These two phenomena are the primary drivers of target damage. A substantially new target vessel design will build on lessons from operational experience and post-irradiation examination of targets.

With the completion of the PPU, the lifetime of the FTS is projected to extend to a total of 60 years, which is approximately 40 years after the completion of the STS. FTS hardware with the highest radiation damage rates is replaceable (e.g., the target module, proton beam window, and inner reflector plug [IRP]). For these components, higher dose rates and their accumulated doses can be addressed with increased operational replacement frequency or relaxation of the material administrative dose limits. However, increased replacement frequency is not an option for permanent components, such as the outer reflector plug (ORP), which may see locally increased dose rates at $1.3 \mathrm{GeV}$. Higher administrative dose limits or more accurate dose rate evaluations should provide the necessary radiation lifetime margins for permanent components.

The PPU FTS Systems goal is to reliably operate at $2.0 \mathrm{MW}$ at $1.3 \mathrm{GeV}$ at $60 \mathrm{~Hz}$. Necessary upgrades to achieve this power level will be defined as early as possible in the CD-1 project phase. Upgrades associated with the PPU beam-capable target module, target high-rate gas injection supply, gas management in the mercury process system, and off-gas systems fall within PPU FTS Systems project scope implementation, as does the addition of ortho-to-para hydrogen convertors in the cryogenic moderator system. Other upgrades determined necessary to ensure reliable $2.0 \mathrm{MW}$ operation of FTS systems will be implemented by SNS operations.

\subsection{OVERVIEW OF FTS SYSTEMS EVALUATIONS AND UPGRADES}

FTS Systems' technical work scope is organized by systems in a manner similar to the existing SNS. The work breakdown structure is graphically shown in Figure 6.1. Within each Level 3 area are activities broadly broken down into evaluating the impact of the higher beam energy and power (which will include specifying required upgrades or operational changes and documentation updates) and upgrades associated with a 2 MW target module and systems supporting target gas injection. There is a related R\&D effort on 
gas injection and efforts to mitigate postulated accidents leading to mercury escaping the service bay. Gas Injection R\&D is described in Section 8.2.

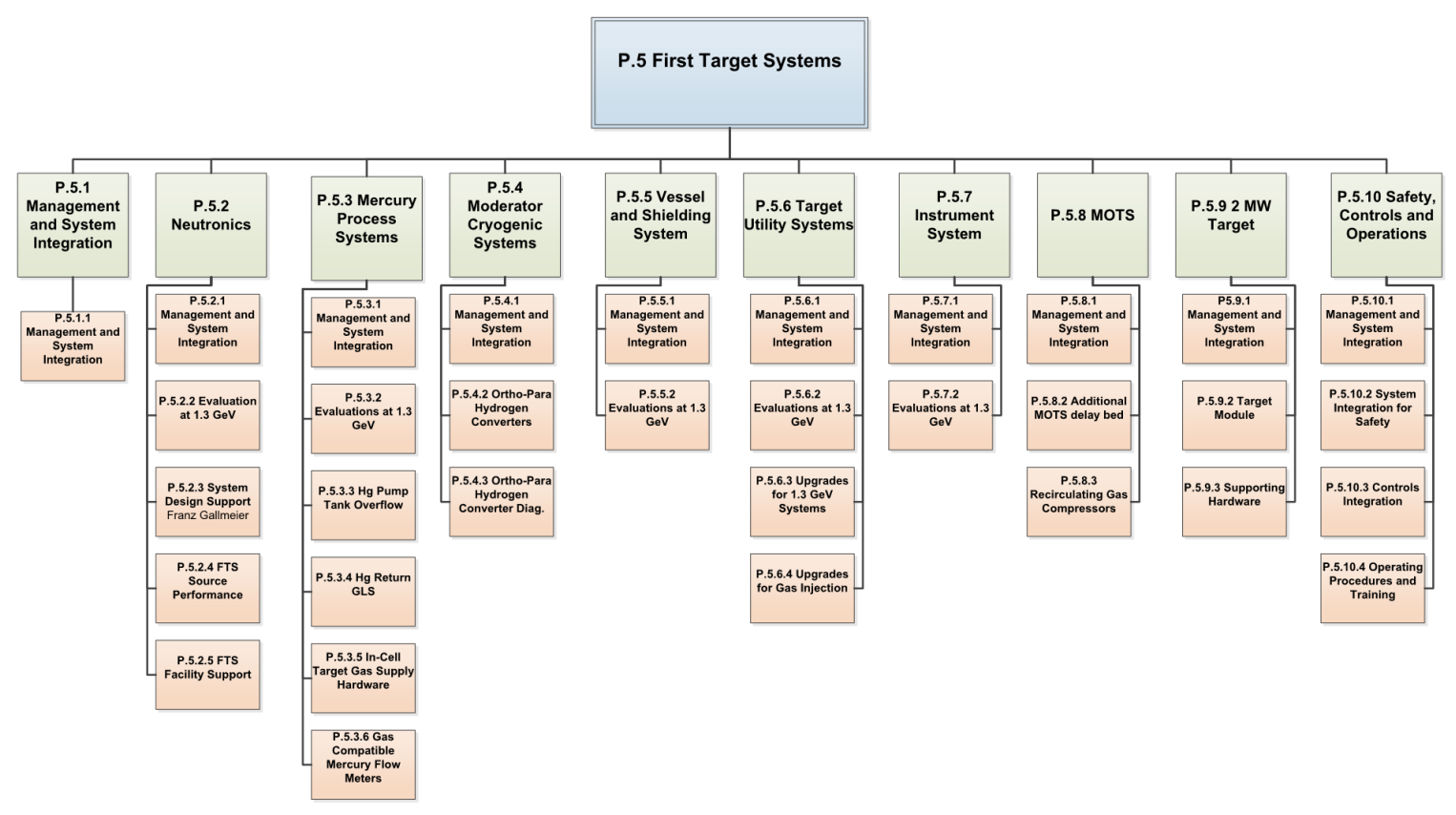

Figure 6.1. FTS Systems Work Breakdown Structure.

Upgrades determined necessary for 2.0 MW operation irrespective to proton energy will be completed by SNS operations. Preliminary analyses have identified some areas, and their completion by operations has been added to the PPU project assumptions document.

\subsubsection{FTS Systems Level 3 Summary Descriptions}

The following items provide summary descriptions of PPU FTS Systems Level 3 work scope.

- Neutronics - Evaluations will determine nuclear power deposition, radiation dose, and damage rates; shielding efficacy throughout the FTS monolith and instruments; and the facility radionuclide inventory for the FTS PPU beam for its extended lifetime to 60 years. These findings will support other systems in conducting engineering analyses and in upgrading the work scope to achieve the required PPU performance.

- Mercury Process Systems - Evaluations will ensure there are sufficient engineering and operational margins in the mercury process systems for the PPU beam and will define necessary upgrades if needed. Upgrades needed to accommodate high-flow target gas injection are part of this project scope. Required operational procedure changes will be implemented.

- Moderator Cryogenic Systems (MCS) - The PPU project scope for moderator cryogenics systems (which excludes the moderator vessels and their transfer lines) includes the addition of catalyst or converter equipment to ensure near $100 \%$ para-hydrogen in the three hydrogen moderator loops. This upgrade will ensure FTS neutronic performance is consistent throughout operating cycles and as 
predicted by neutronics analyses. Associated operational procedure changes will be developed and implemented. Several reliability and heat removal capacity improvements to the MCS have been identified for $2 \mathrm{MW}$ operation, which will be funded by SNS operations.

- Reflector Vessel and Monolith Shielding - These evaluations will ensure there are sufficient engineering, radiation, and operational margins in the FTS vessel and monolith shielding systems for the PPU beam and define necessary upgrades if needed. Hardware upgrades are not foreseen. Evaluations will include the ORP, core vessel, and beam line shutters. Changes to the subsystem cooling utility requirements and control set points may be specified. Any required operational procedure changes will be developed and implemented. Furthermore, increasing the radiation damage limit on the ORP appears necessary. Preliminary analysis indicates that after the extended lifetime, portions of the ORP will reach 13 displacements per atom (dpa). A justification to increase the damage limit beyond the original 10 dpa appears feasible.

- Target Utility Systems - This evaluation will ensure that there are sufficient engineering and operational margins in the FTS target utility systems for the PPU beam and will define necessary upgrades as needed. Utility changes will be coordinated with the needs of other subsystems. Assumptions have been made with regard to the extent of required utility upgrades due to the change in energy distribution throughout the FTS, which will be resolved as systems evaluations progress. Required operational procedure changes will be implemented. Target utility systems is also responsible for adding high-flow gas supply capabilities as required by the $2.0 \mathrm{MW}$-capable target for equipment outside the service bay or the MOTS equipment room.

- Instrument Systems - This evaluation will ensure there are sufficient engineering, radiation, and operational margins in FTS instrument systems with the PPU beam and define upgrades, if needed. The primary focus is on core vessel inserts (CVIs), especially in the forward proton beam direction beam lines. The CVIs are part of a credited boundary, so relevant engineering analyses and safety documents will be updated. Any new cooling requirements will be coordinated with target utilities, and operational procedure changes will be made.

- Mercury Off-gas Treatment Systems (MOTS) - High-flow gas injection is expected to effectively sweep activated spallation gases out of the mercury process system. To limit the dose to MOTS equipment and personnel, the addition of a second carbon delay bed is planned. In addition, the present vision for high-flow gas injection sees gas recirculation as necessary; and the preferred approach is to locate compressors in the MOTS equipment room, where they will draw from the treated off-gas. Because of this location and close integration with MOTS equipment, these compressors will be covered in the PPU MOTS project scope. New operating procedures will be generated.

- $2 M W$ Target-This work scope designs and delivers a target module ready for the PPU beam. The target will incorporate high-flow gas bubblers and a protective gas wall at the inner target beam entrance window based upon guidance from PPU R\&D on target gas injection, results of the low-flow gas injection initial implementation (GI3) effort, and progress achieved through near-term target operations. Other improvements for fatigue life margin and general robustness will be incorporated into the design.

- Safety, Controls, and Operations - These system cross-cutting activities are essential to operational readiness for PPU beam on the FTS. Hazards associated with high-flow gas injection require an integrated systems approach to developing designs that sufficiently mitigate accident risks and for which safety authorization must be obtained. Control — whether related to gas injection or not—-touch 
on nearly all FTS Systems project scope. Operating procedures and required training updates will be made ready for operations with the system changes, and new equipment will be installed for PPU.

There is no PPU work scope for moderators or the IRP. The Instrument Source Division of SNS will provide an IRP (\#3) that will be fully compatible with PPU beam energy and power.

\subsubsection{High-Flow Target Gas Injection}

Gas injection addresses two target lifetime vulnerabilities - insufficient fatigue life from the cyclic loading caused by beam pulse pressure waves, and cavitation erosion also originating from beam pulse pressure waves. The high-flow gas injection envisioned for the mercury target requires substantial work across FTS systems, besides the target module. Mercury process systems; target utility systems; MOTS; and safety, controls, and operations all have significant project scope to enable high-flow gas injection. The broad plan and basis for SNS target gas injection-from intial steps under SNS operations to maximum deployment - is outlined in the 2016 Conceptual Design Report for Mercury Target Gas Injection [1]. As described in the PPU 2 MW Target Development Plan, [2] the target and supporting systems design will incorporate developments from PPU R\&D, from SNS operations vis-à-vis the SNS Target Management Plan [3], and from the GI3 [4] project.

As power increases, the fatigue and cavitation vulnerabilities increase disproportionally, requiring effective countermeasures. Gas injection - in two forms - has very good prospects to provide substantial life improvement when properly developed and deployed in the target. The two forms of gas injection are

- Small gas bubbles injected at high gas flow rates and dispersed throughout the target

- A protective gas wall or bubbly curtain covering known areas of high cavitation damage, specifically the middle portion of the inner beam entrance wall of the mercury vessel, on the wall surface facing the bulk mercury volume.

The small gas bubble generators should generate bubbles of about $150 \mu \mathrm{m}$ in diameter or less in mercury with an injected gas volume fraction of about $0.5 \%$ (about 6 SLPM for a 300 gpm mercury flow rate). It has been shown numerically [5] that a bubble population of this size can significantly reduce vessel pulse stresses away from the incident beam spot. The best prospects for achieving bubble populations of this specification are with swirl bubblers - the technology used in the Japan Proton Accelerator Research Complex (J-PARC) mercury target. Online measurements of the J-PARC target vessel confirmed vibration reduction with a swirl bubbler to about one-third that without bubbler operation (Figure 6.2). 


\section{Bubbling effect on vibration}

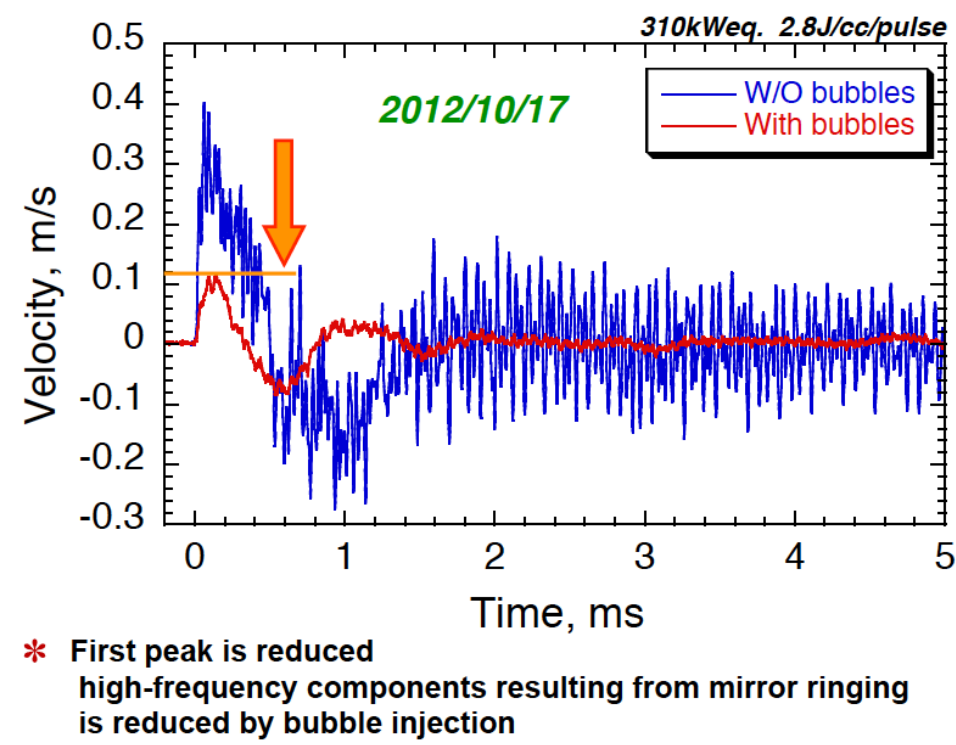

Figure 6.2. Vibration data from Japan Spallation Neutron Source (JSNS) target illustrating the attenuating effects of gas bubble injection. From T. Naoe, 2012 SNS-JSNS Collaboration meeting.

The protective gas wall should be such that (a) the middle half of the inner beam entrance window wall of the mercury vessel is covered by a continuous gas blanket, or at least a bubbly blanket, (b) the gas wall does not affect the small gas bubble injection, and (c) its injection rate does not exceed 10 SLPM. The goal for time-averaged gas coverage in this region is at least $60 \%$.

Recirculating compressors will be used to limit helium consumption and reduce stack emissions. They should be capable of injecting up to 20 SLPM at 60 psig and lower flow rates at up to 100 psig. The location of the compressors will be chosen based on the R\&D findings and GI3 progress; presently the MOTS equipment room is preferred. Dual-mode gas supply operating capability is needed for system reliability and flexibility; a once-through gas supply is foreseen for this purpose. Requirements will be provided to and implemented by MOTS and target utility systems.

The target module vent line will be redesigned to remove as much gas as possible in the return channel of the target. R\&D will be performed at the Target Test Facility (TTF) to improve and evaluate its efficiency. However, additional gas removal is necessary to ensure that the amount of gas flowing into the heat exchanger is of the same order as that in the GI3, or about $0.04 \%$ in injected gas. The development of a gas liquid separator for the mercury return pipe is planned in the R\&D phase; requirements will be provided to and implemented by the mercury process systems. The need for a mercury pump tank overflow is also foreseen, to protect against liquid mercury overflowing the tank and potentially escaping the service bay. Its design and installation are part of the mercury process systems.

The MOTS will be upgraded to handle a steady gas flow rate of 20 SLPM and a higher transient gas flow rate that could be caused by the release of a large gas pocket in the pump tank. A second carbon delay bed will be installed in the gold amalgamation room (GAR) to increase the delay of vented spallation hot gases and provide the ability to swap out a carbon canister when needed. 
Given the potential hazards associated with high-rate target gas injection, the overall safety of the integrated system designs control and envisioned operation must be reviewed and approved before completion.

\subsection{DETAILED WORK SCOPE DESCRIPTIONS}

\subsubsection{Target Station Neutronics Evaluations and Upgrade Support}

At a constant power of $2.0 \mathrm{MW}$, the proton current incident to the target will be decreased from $1.25 \times 10^{16}$ to $0.96 \times 10^{16}$ protons/s by increasing the proton energy from 1.0 to $1.3 \mathrm{GeV}$. The neutron yield per proton on thick high- $Z$ target scaling is approximately linear with the proton beam energy, according to Hilscher [6], so the neutron source strength remains constant with the beam energy at constant power for the energy increase considered here.

Nevertheless, the proton energy increase will cause redistribution of the neutron flux field:

- The proton range (i.e., the distance to which a proton can penetrate into bulk materials and continuously lose energy through ionization until it stops) into the target depth will increase from 45 to $64 \mathrm{~cm}$;

- The high-energy fraction of the secondary neutron source is more (proton beam) forward directed, causing higher energy deposition and higher material damage in the forward direction.

Both effects increase the energy deposition in the transition region of the target by a factor of 10 for $1.3 \mathrm{GeV}$ for the same power on the target. While the absolute heating rates will still be more than an order of magnitude lower compared with the peak heating rates in the front part of the target, the PPU target design must account for this change.

Initial Monte Carlo (MC) transport analyses from a $1.3 \mathrm{GeV}$ proton beam over the target and nearby IRP regions were performed to assess component energy deposition, peak material radiation damage, moderator performance, and target radionuclide production [7]. These calculations confirmed that most quantities of interest outside the target module remain unaffected by the proton energy increase. Representative power deposition around the target and IRP was shown for both proton energies in Figure 6.3. For those quantities and regions dominated by proton fluxes, such as proton beam window material radiation damage, damage rates decrease with the higher energy and lowered proton current density compared with $2.0 \mathrm{MW}$ and $1.0 \mathrm{GeV}$ operation. Peak heating rates of the proton beam window and the target nose see a similar reduction in proton-induced components, but heating rates from secondary particles such as neutrons are unchanged. For quantities and regions solely dependent on neutron radiation - such as moderator or IRP heating and damage rates - the proton energy increase has little impact for the same operating power.

Further MC studies expanded the investigation domain into the ORP with similar results to those seen for the IRP except for one region: the ORP locally experiences increased heating and radiation damage rates by a factor of three owing to the proton energy change in the forward beam direction [8]. Acceptable temperatures and thermal stress will be verified as part of other PPU FTS evaluation work scope, and required cooling changes will be defined if needed. Over the updated projected FTS lifetime of 60 years, the section of the ORP adjacent to the target is projected to accumulate $13 \mathrm{dpa}$ in radiation damage. This projection considers the material radiation damage incurred over the last 10 years and assumes operation for the next 10 years at $1.4 \mathrm{MW}$ and $1.0 \mathrm{GeV}$, followed by 40 years of operation at PPU conditions $(2.0 \mathrm{MW}$ and $1.3 \mathrm{GeV})$. This rate exceeds the original design basis limit of $10 \mathrm{dpa}$ and the current administrative limit of $12 \mathrm{dpa}$ for the replaceable target module. The dpa limit for the ORP needs to be 
revised, which likely can be justified as the facility accumulates more target material irradiation data and gains confidence with exposing stainless steel to higher doses.

Although the recent large-view, low-resolution MC analyses indicated that the change in proton beam energy generally does not affect the conservative, simplified heating assumptions that were the original design basis of FTS component thermal analyses, we will verify this finding with dedicated, detailed energy deposition analyses for the target station components.

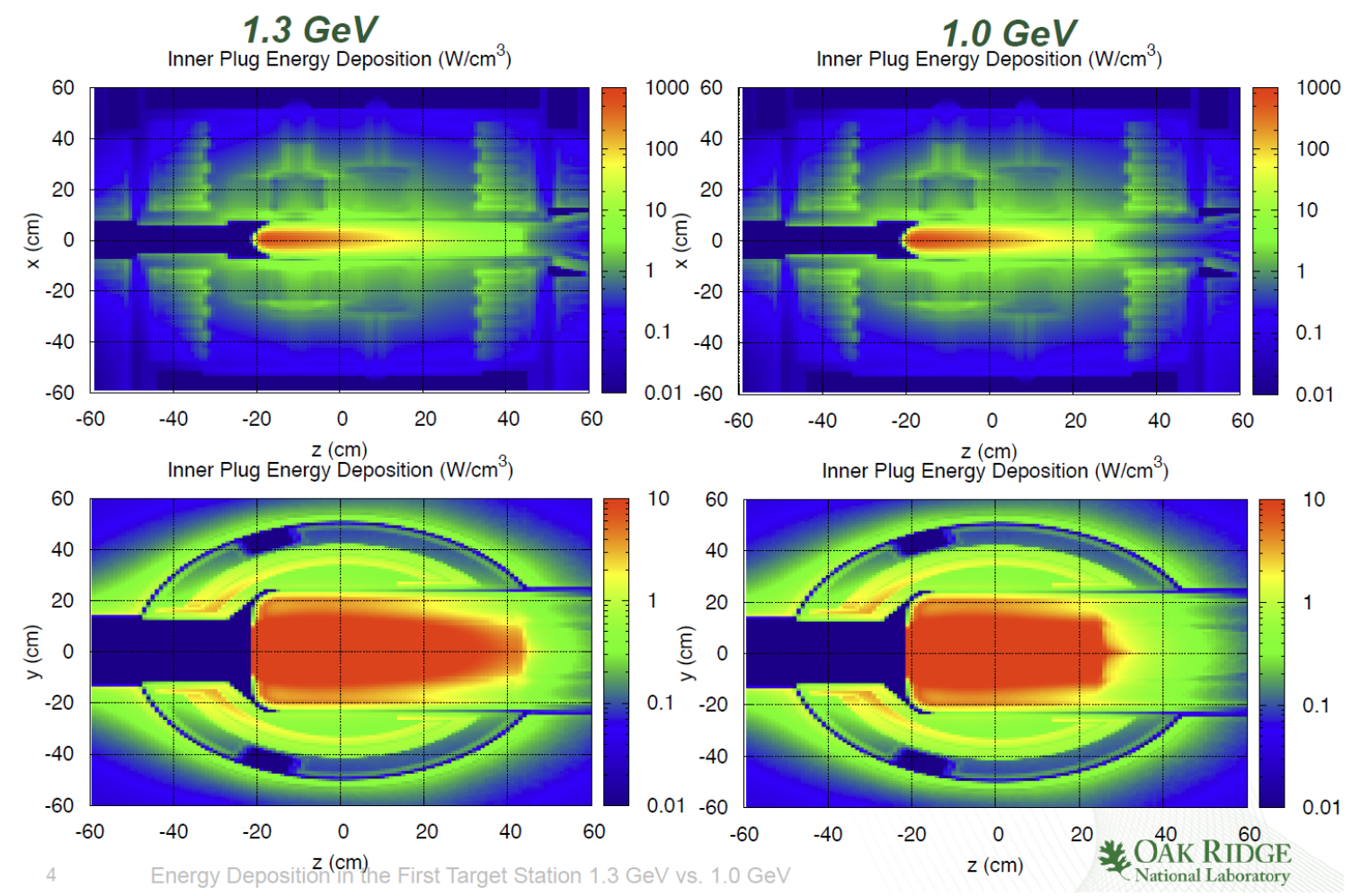

Figure 6.3. Power deposition comparison in FTS for 1.3 and $1.0 \mathrm{GeV}$ at the target and IRP.

Some radiation transport calculations have also been recently performed to assess the shielding efficacy of the FTS target monolith. The dose rates outside of the monolith with $1.3 \mathrm{GeV}$ protons at $2.0 \mathrm{MW}$ power on the target are essentially unchanged over the $1.0 \mathrm{GeV}$ case, and they are a factor of three below the design goal of $0.25 \mathrm{mrem} / \mathrm{h}[9]$.

Heating and radiation damage to other FTS systems will be confirmed, especially for the forward beam direction, including

- target cart and shielding toward target service

- core vessel target port region

- $\quad$ shielding of forward-directed beam lines $8,9,10$, and 11 .

The radionuclide inventory for the target mercury, which is assumed to be used for the life of the facility, was recently analyzed assuming $1.0 \mathrm{GeV}$ proton beam exposure at $1.4 \mathrm{MW}$ power for 5,000 hours per year for 20 years, followed by exposure to $1.3 \mathrm{GeV}$ protons at $2.8 \mathrm{MW}$ power for 40 additional years again for 5,000 hours per year. As this nuclide inventory will be the basis of the environmental impact statement (EIS), conservatively high power levels were assumed. The mercury activity at end of life 
corresponds with the mercury activity obtained for the original SNS at $1.0 \mathrm{GeV}$ proton energy, $2.0 \mathrm{MW}$, and 40 years of operation at 5,000 hours per year after scaling by the ratio of powers [10].

Assessments of the end-of-life bounding inventories of the target module and the proton beam window module are in preparation and will be available shortly for reestablishing their waste classification; changes are not expected. If significant changes are seen in these over the $1.0 \mathrm{GeV}$ case, an assessment of the IRP will also have to be performed as part of the project effort.

Any further neutronic analyses beyond that done to date for the completion of the EIS or facility safety documentation will be completed as part of this work scope.

The PPU strives to reach at least the projected moderator performance as reported by Lu for the FTS at 2.0 MW with $1.0 \mathrm{GeV}$ protons [11]. This estimate assumed the hydrogen moderators are fed with $100 \%$ para- $\mathrm{H}_{2}$ and the IRP is cooled with heavy water $\left(\mathrm{D}_{2} \mathrm{O}\right)$. To ensure this condition is consistently maintained in the moderator, the hydrogen loops require the addition of catalyst devices that are planned as part of the PPU. Gains of $2 \%$ and $8 \%$ in the time-averaged brightness of the upstream and downstream moderators, respectively, can be expected if the proton energy is increased from 1 to $1.3 \mathrm{GeV}$ with the same para- $\mathrm{H}_{2}$ and $\mathrm{D}_{2} \mathrm{O}$ conditions applied.

Presently, the decoupled hydrogen moderator experiences $20 \%$ performance degradation at high power operation for $3 \AA$ neutron wavelengths compared with the expected linear scaling with proton power. For shorter-wavelength neutrons, this nonlinearity is less pronounced. This issue is partly being addressed independent of the PPU. Current thinking is that the degradation may be caused by the reduction of hydrogen density in the moderator resulting from nuclear heating. If verified, this could be remedied by increasing $\mathrm{LH}_{2}$ flow or by modifying the internal flow geometry inside the decoupled hydrogen moderator. No degrading effects have been seen so far in the coupled hydrogen moderators or in the decoupled water moderator.

Neutronics support to other FTS systems will be provided for thermal engineering analysis, for radiation damage effects, and to ensure that PPU neutronics performance goals and reliable operation will be achieved.

\subsubsection{Mercury Process System Evaluation and Upgrades}

The mercury process system includes the mercury pump, heat exchanger process piping, storage tank and collection basin, target carriage, and related controls. It does not include the target module. Figure 6.4 shows an overview of the target process bay with the mercury process system and associated shielding (left) and the mercury loop components (right). 

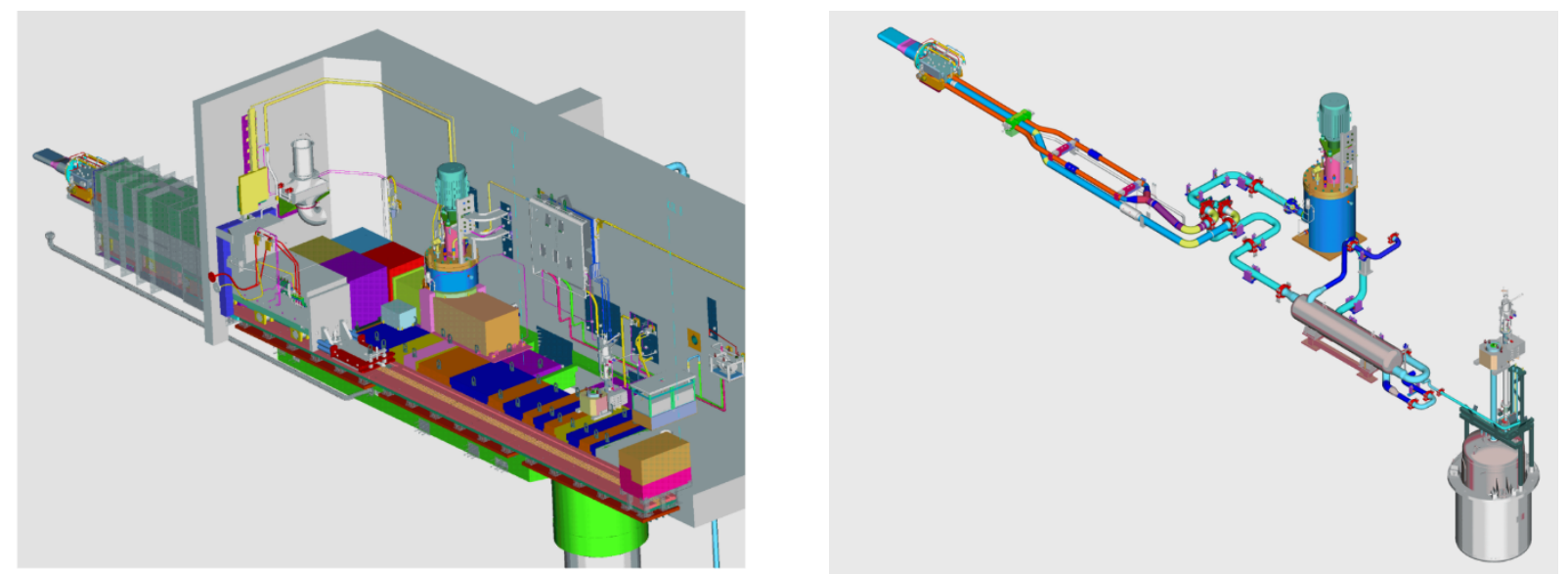

Figure 6.4. Target process bay (left) and mercury process loop (right).

These systems will be evaluated for PPU operation at $1.3 \mathrm{GeV}$ and $2.0 \mathrm{MW}$ with a facility life extension of 20 years over the initial design requirements (for a total of 60 years). The gas injection impact to the mercury process systems work scope will be developed under R\&D (Sections 8.2) and implemented under Mercury Process Systems (Section 6.3.2.1). Foreseen are a mercury pump tank overflow and a gas liquid separator in the mercury return pipe at the back of the carriage.

The FTS mercury pump was designed and tested for the $2.0 \mathrm{MW}$ and $1.0 \mathrm{GeV}$ condition of operation at $400 \mathrm{rpm}$ with a nominal mercury flow of $380 \mathrm{gpm}\left(1.44 \mathrm{~m}^{3} / \mathrm{m}\right)$. This corresponds to a bulk average mercury loop temperature rise of $30^{\circ} \mathrm{C}$ through the target. The heat exchanger was designed for $1.4 \mathrm{MW}$ energy deposition in the mercury, which is a conservative fractional estimate for the $2.0 \mathrm{MW}$ beam on target. Approximately 65\% energy deposition in the mercury has been measured from the water loop enthalpy change during 1.0 MW operation. No significant difference in total heat deposition in the mercury is expected for the $1.3 \mathrm{GeV}$ case, but this will be verified. The distribution within the target module shifts downstream, but the total deposition will be very similar.

The mercury pump mechanical components are assumed to be functional for the life of the facility, but a spare pump has been procured. Some internal features of the spare pump have been changed from the original, and those changes now require modifications for compatibility with gas injection. It is assumed those modifications will be completed using SNS operating funds. The pump motor is expected to experience radiation damage to its insulation as a result of the nominal background of $200 \mathrm{R} / \mathrm{h}$ and is expected to be replaced as part of normal operations. The heat exchanger is also expected to last the life of the facility, but a spare heat exchanger is available if needed. Without consideration for target gas injection, no changes are expected for the piping, storage tank, or collection basin. No fatigue or erosion limits have been identified that would limit component lifetimes. The process loop instrumentation and controls are expected to be the same, aside from set point changes and excluding potential effects of gas injection.

The target carriage is the only sub-system that might require new engineering analysis. The $1.3 \mathrm{GeV}$ beam energy will increase the heating in the front of the carriage on the component supporting the target (a.k.a. the "diving board") and the shielding. Figure 6.5 shows the results of the Title II thermal analysis for $2.0 \mathrm{MW}$ operation with $1.0 \mathrm{GeV}$. Temperature peaks were about $160^{\circ} \mathrm{F}$. However, a clear temperature limit was not established in the original evaluations.

The evaluation of the carriage for $1.3 \mathrm{GeV}$ operation would include developing the detailed neutronic heating rates (Section 6.3.1) and comparing them with the $1.0 \mathrm{GeV}$ results. If found to be worse than the 
simplified bounding heating rates used in the original design basis, the revised heating rates will be applied to new models to estimate temperatures, displacements, and thermal stresses. The model may include CFD estimates of forced air flow around the carriage. Figure 6.6 shows the flow from tubes near the front of the carriage tunnel estimated during Title II.

Any required upgrades to the mercury process systems exclusive of those related to target gas injection will be designed, fabricated, and installed under operations funding. If the thermal evaluation indicates excessive temperatures, any detailed design for carriage modifications will be completed. At this time, the need for changes is considered unlikely.

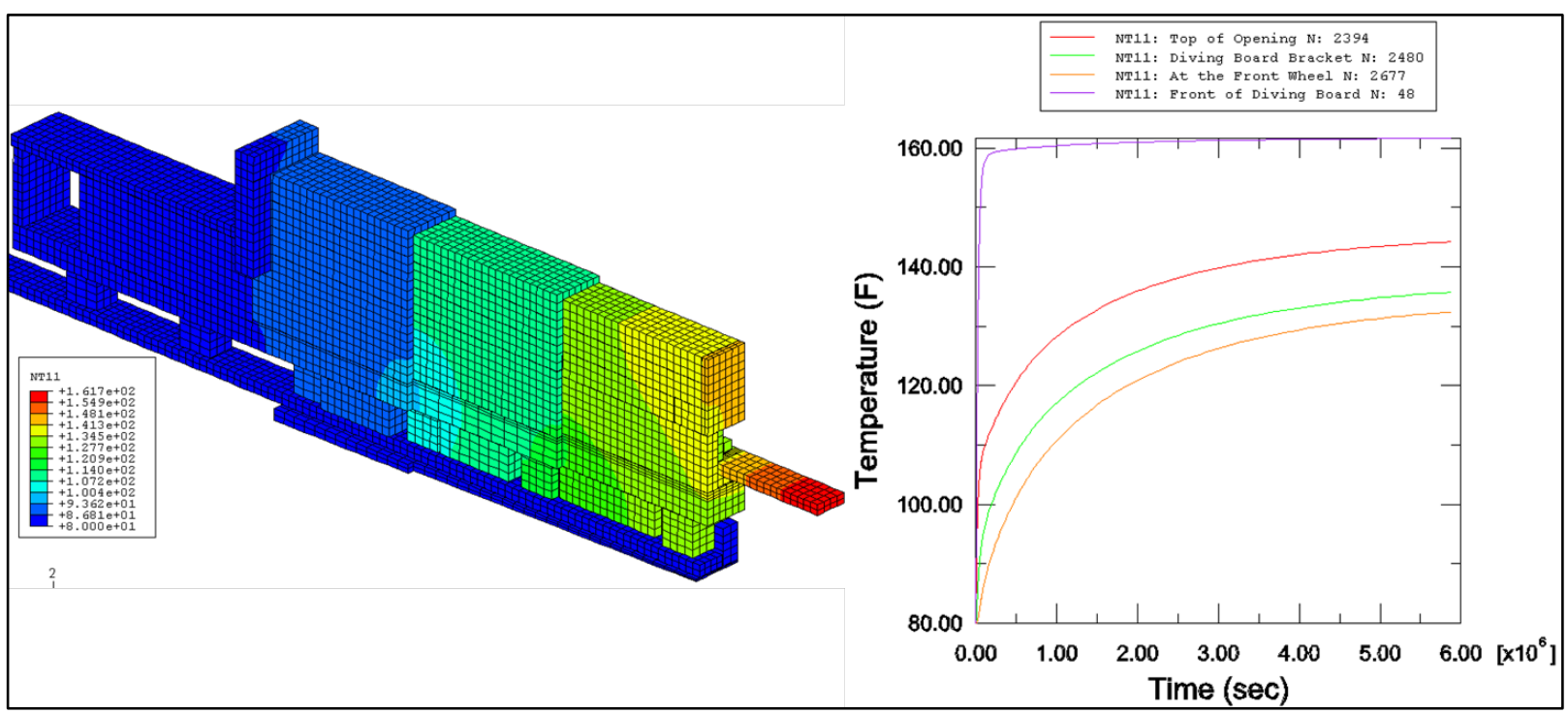

Figure 6.5. Target carriage temperature profile at $2.0 \mathrm{MW}$ with a $1.0 \mathrm{GeV}$ beam.

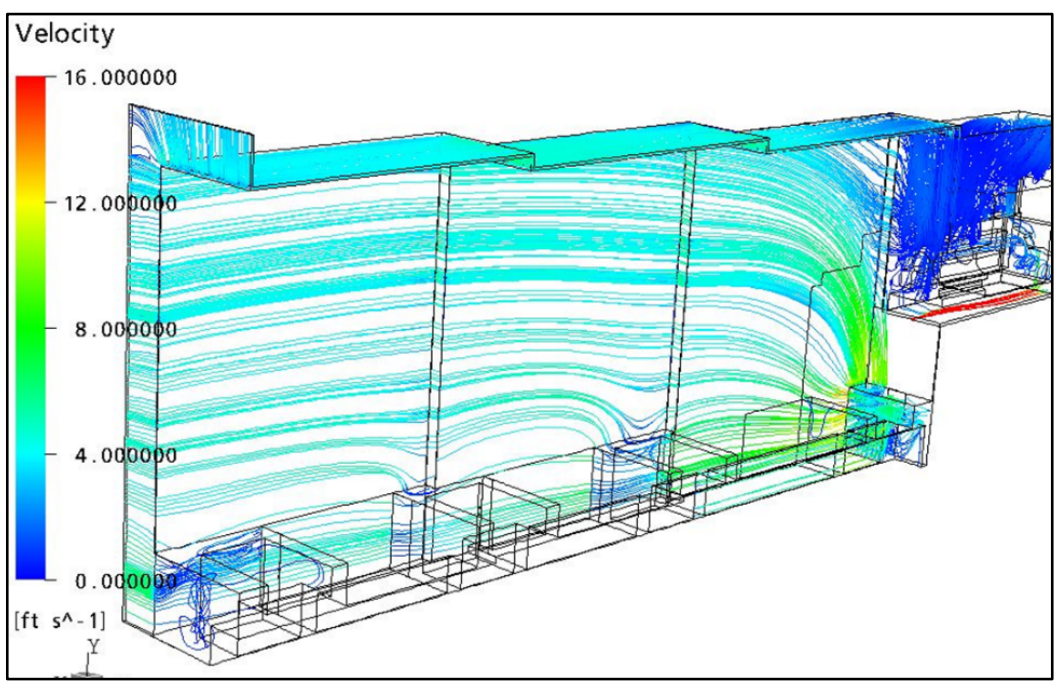

Figure 6.6. Steady-state streamlines through the air flow around the target carriage. 


\subsubsection{Mercury Process Loop Modifications for High-Rate Target Gas Injection}

High-rate target injection is expected to have adverse effects on the gas in the mercury process loop that will have to be mitigated. As a result of the configuration of the process piping and heat exchanger, gas is likely to accumulate in various regions (Figure 6.7).

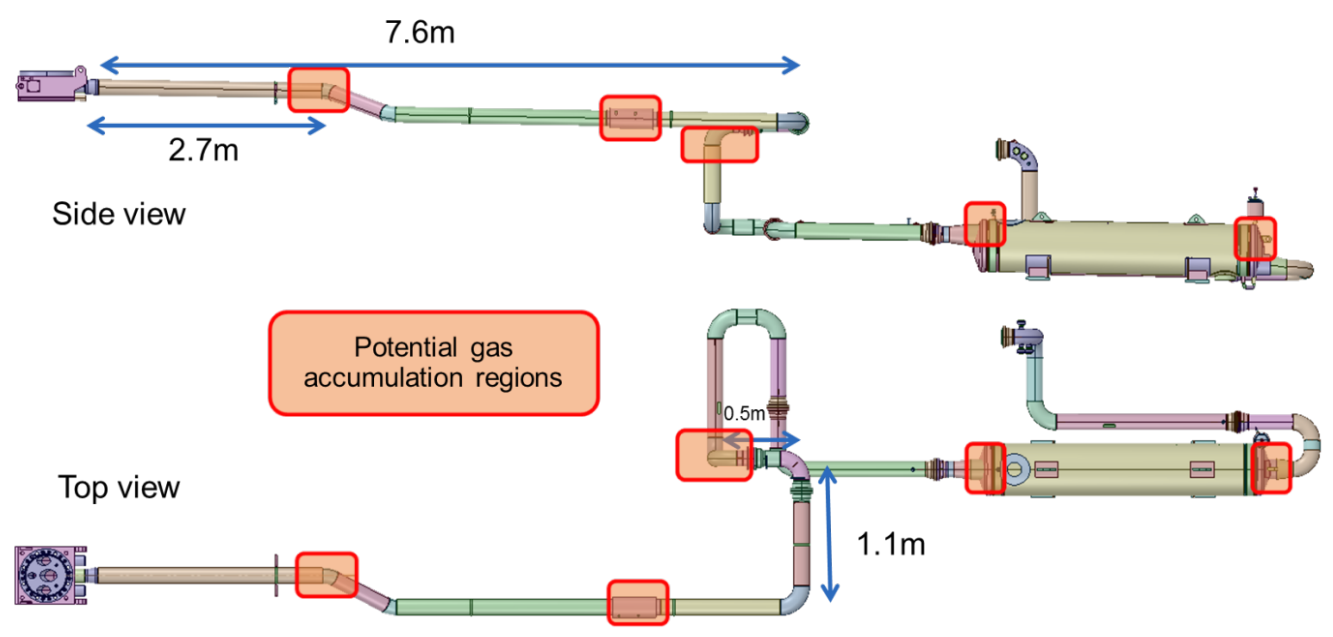

Figure 6.7. Side and top views of the mercury process piping and heat exchanger showing regions that will likely accumulate gas as it leaves the target module.

Unmitigated, this accumulated gas is expected to

- displace mercury from the pipes and heat exchanger, raising the level in the pump tank enough to fill it and send liquid mercury out the gas vent line and into the MOTS

- increase flow resistance, thus increasing pump power

- partially foul tubes in the heat exchanger and reduce its heat removal efficiency

- displace mercury in the heat exchanger bulkheads and impose a pressure gradient across the tube set that could lead to preferential flow bias of the top or bottom with an associated thermal gradient not considered in the heat exchanger design basis

- create gas pockets at flow-measuring venturi tubes that will increase the error in mercury flow readings

Tests done at the TTF - a full-scale mercury test loop configured similarly to the SNS mercury loopdemonstrated that, at gas injection rates about half that envisioned for PPU target gas injection, the mercury level in the pump tank continued to rise until reaching the tank overflow nozzle [12]. The SNS pump tank does not have a functioning overflow, and under high rates of gas injection, liquid mercury is likely to start going up the vent line that leads to the service bay gas panel and the MOTS outside the service bay unless sufficient mitigations are deployed. The other adverse effects listed above have also been observed in the TTF, except for the heat exchanger fouling, as the TTF does not have a prototypic heat exchanger.

Prevention of liquid mercury escaping the service bay is necessary for facility safety. The envisioned modifications to the process loop to mitigate this accident include two features that will require difficult remote handling work: 
- The overflow capability of the mercury pump tank will be modified as shown in Figure 6.8

- An aggressive gas-liquid separation (GLS) fixture will be added in the return pipe elbow at the end of the carriage (Figures 6.9 and 6.10).
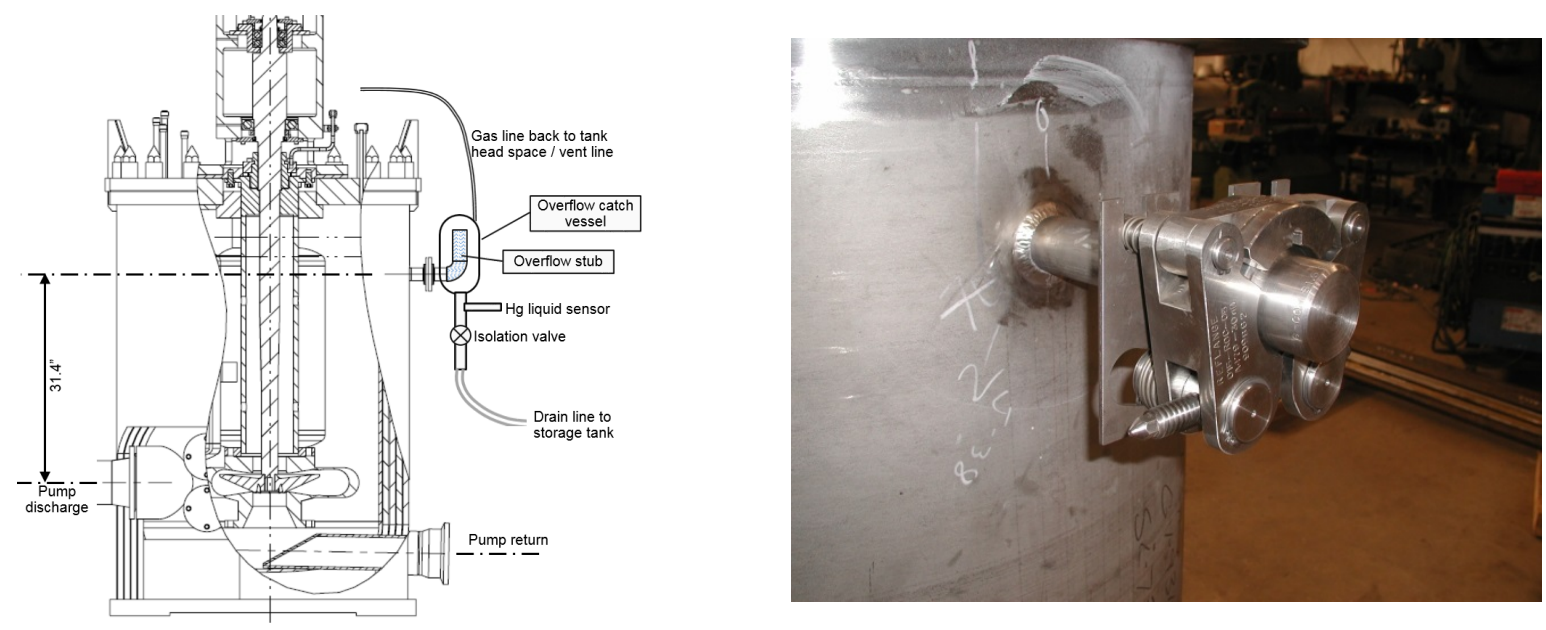

Figure 6.8. The mercury pump model shows the upper side of the nozzle at $31.4 \mathrm{in}$. above the discharge nozzle elevation. A concept for an overflow valve attached to the nozzle has been proposed. The nozzle is presently capped as shown on the right, but it is also covered with a shield block that must be removed if an overflow is to be installed.

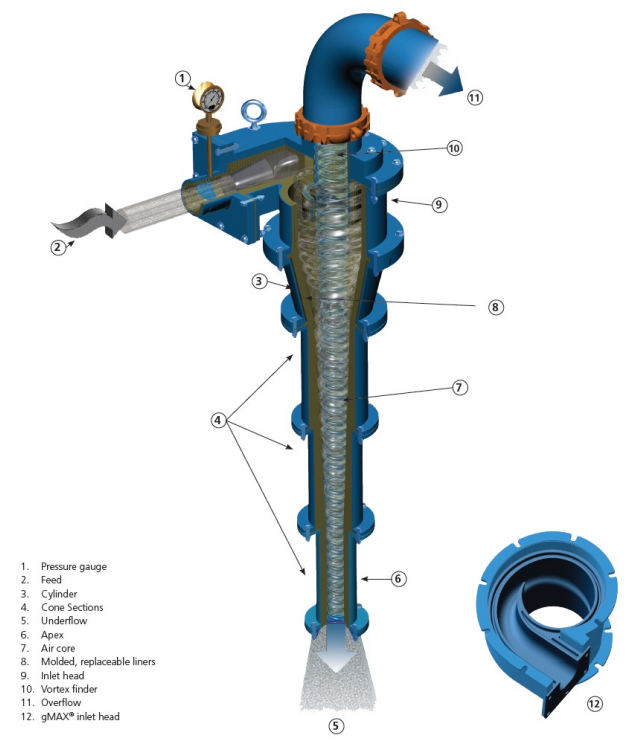

Figure 6.9. Example hydro cyclone design type that might be adapted to mercury-gas separation at the return pipe elbow at the end of the carriage. From FL Smidth KREBS Co. 

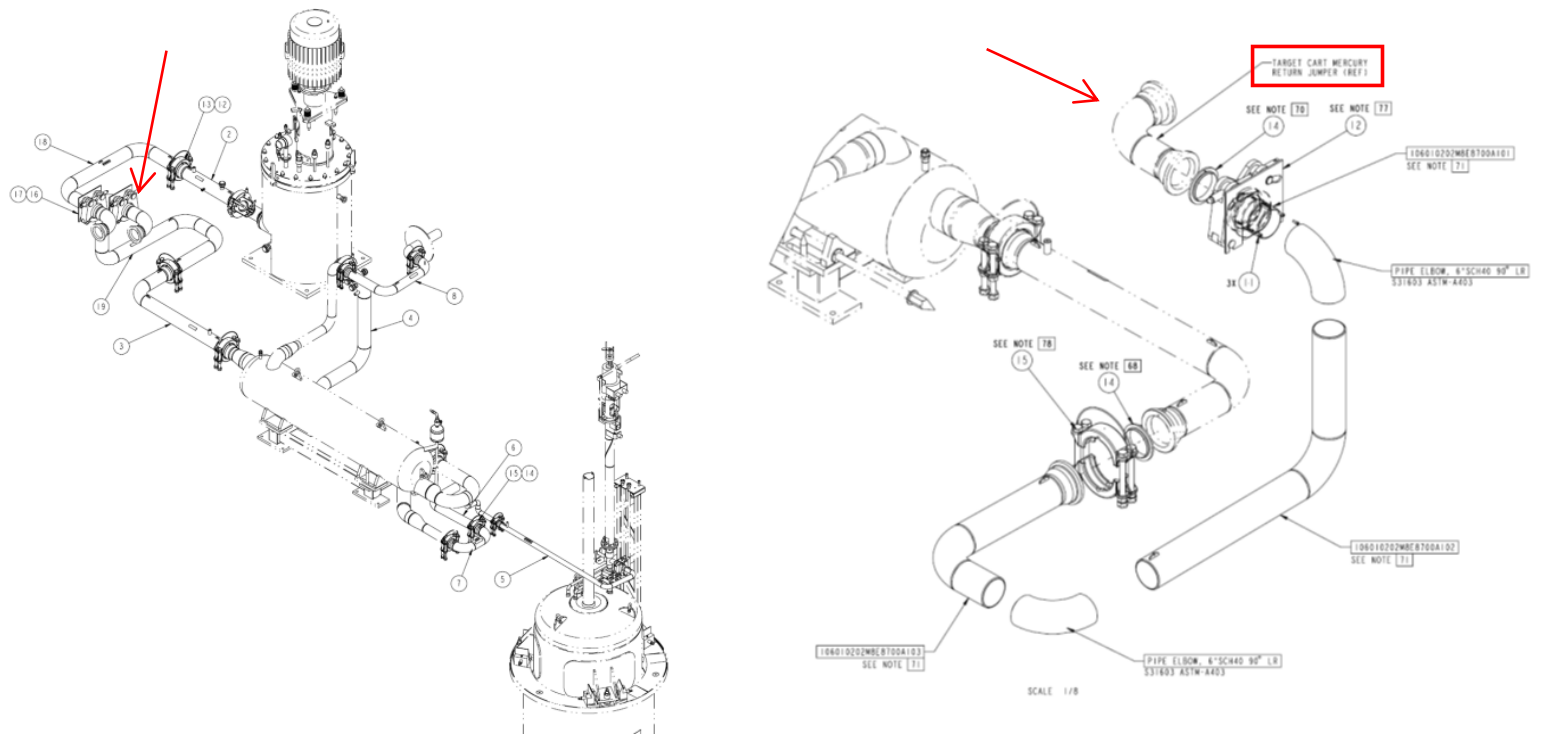

Figure 6.10. Mercury process loop piping. The $90^{\circ}$ return pipe at the end of the carriage might be replaced with a cyclonic gas-liquid separator or other device.

Depending on the results of R\&D and lessons learned from low-rate target gas injection associated with the GI3 effort, it is possible these modifications may not be necessary; or other more readily deployable solutions may become apparent. But it is presently assumed that these new features are required in the scope of PPU. It is also assumed that the heat exchanger will not need to be replaced or modified.

Remote handling procedures will be developed for the required modifications, and the necessary installation tools and fixtures will be designed and fabricated.

Another planned mercury process loop modification is the addition of new or upgraded flow sensors that will be unaffected by gas injection. This technology is expected to result from R\&D efforts.

Final requirements for mercury process loop modifications will come from R\&D and safety integration regarding high-flow target gas injection, but the modifications mentioned are the major planned improvements. This work scope includes the design, fabrication, and installation of these modifications in coordination with other non-gas-injection related upgrades. Required changes to relevant process loop instruments and controls will be specified to the controls group. Required operational procedure changes will be developed and implemented.

\subsubsection{Moderator Cryogenic Systems Upgrades}

Ortho-to-para hydrogen convertors will be added to the MCS as part of the PPU (note that the MCS is a subset of the entire cryogenic moderator system and does not include the moderator vessels or transfer lines). This scope is included in the PPU to ensure FTS neutronic performance is consistent throughout operating cycles and as predicted by neutronics analyses. It is also key aspect of the high-brightness neutron source technology needed for the STS project, and demonstration in PPU provides a basis for the STS implementation. These units will be added to the three individual loops by modifying the existing hydrogen pump module. The convertors will represent an increase in the hydrogen inventory, as well as an additional pressure drop to the hydrogen fluid loops. Additional analysis will be needed to determine the impact on circulator performance caused by the increased loop pressure drop. The individual convertor vessels will be approximately a meter in length and filled with a catalyst (e.g., ferrous oxide). 
The vessels will be fitted into the flow paths for the moderator loops. The vacuum vessel envelope of the pump module will need to be enlarged to accommodate the convertor vessels. This is not expected to have a significant impact on the hydrogen utility room. The additional hydrogen inventory will be considered in updates to the facility safety basis. Associated with the convertors will be the installation of online, in situ diagnostics to measure the fractions of ortho- and para-hydrogen to confirm convertor performance. Increasing the accelerator beam power to FTS to $2.0 \mathrm{MW}$ will have an impact on the existing MCS. An extrapolation of the operational data projects that the current cryogenic system would barely be able to maintain the proper system temperatures with a reserve refrigeration capacity of $1 \mathrm{~kW}$ only if the system is working at its best. This amount of reserve capacity is not sufficient for reliable operation of the MCS.

Other reliability risks have been identified and will only increase with $2.0 \mathrm{MW}$ operation. A set of improvements are proposed to improve MCS reliability. These include

- increased oil removal capability

- an additional helium compressor

- efficiency improvements of the helium heat exchanger

- helium refrigerator insulation vacuum system upgrades

- repair and enlargement of the helium-to-hydrogen heat exchangers

- moving hydrogen cylinders outside for safety

These improvements will be implemented under SNS operating funding, most likely as an Accelerator Improvement Project. It is assumed that any necessary improvements to ensure MCS reliability under PPU beam will be completed by the time of PPU operational readiness.

\subsubsection{Evaluations of Reflector Vessel and Monolith Shielding Systems}

This scope ensures there are sufficient engineering, radiation, and operational margins in the FTS vessel and monolith shielding systems, and defines any necessary utility or operational changes.

In the context of PPU FTS Systems, vessel and monolith shielding systems consist of the ORP; the core vessel, including the proton beam port and the target port; the proton beam window; the beam line shutters; and the monolith shielding. Figure 6.11 shows a cross section of the monolith, including the vessel and monolith systems along with the IRP and target, which are discussed in other sections.

These systems will be evaluated with heating and radiation damage for PPU operation at $1.3 \mathrm{GeV}$ and 2.0 MW and with a facility life extension to 60 years.

Initial neutronics analysis shows that, in general, the heat deposition in the vessel and monolith shielding systems from a $1.3 \mathrm{GeV}$ proton beam will be comparable to the $1.0 \mathrm{GeV}$ beam. However, local increases in heat deposition by up to a factor of two to three in the region surrounding the target transition and rear body are indicated, which will require more detailed analysis. New detailed analyses seem unnecessary for the proton beam window and its associated shielding, the core vessel proton beam port, and the bulk monolith shielding. The existing analyses, relevant operational data, and updated $1.3 \mathrm{GeV}$ heat deposition results will first be reviewed to ensure that no further detailed analysis is required for these regions.

The regions that appear to be most impacted by the locally increased heat deposition from the $1.3 \mathrm{GeV}$ proton beam energy are the ORP around the target and the core vessel target port. The original ORP thermal analysis shows a maximum temperature of $94^{\circ} \mathrm{C}$ around the target port with a $2.0 \mathrm{MW}, 1.0 \mathrm{GeV}$ proton beam, as seen in Figure 6.12. Despite the substantial margin in the previous analysis, a factor of two to three increase in the local heat deposition rate could cause overheating and boiling issues in this region. Preliminary neutronics analysis further projects the integrated radiation damage around the target 
port of the ORP to reach 13 dpa because of the increased damage rate and 20 year extension of lifetime; a justification for increasing the original design basis limit of 10 DPA will have to be developed. The original core vessel target port analysis shows a maximum temperature of $184^{\circ} \mathrm{C}$ away from the directed cooling near the inflatable seal interface, as seen in Figure 6.12. Although the most severe increase in heat deposition rates will be near the well-cooled inflatable seal interface, overheating elsewhere in the target port is possible. The heat generation rates for the PPU beam will be applied to conjugate heat transfer CFD models of the components to estimate temperatures and potential for coolant boiling. If significant differences from the $1.0 \mathrm{GeV}, 2.0 \mathrm{MW}$ thermal results are seen, the thermal stress analyses will be revised based on the new temperature profiles.

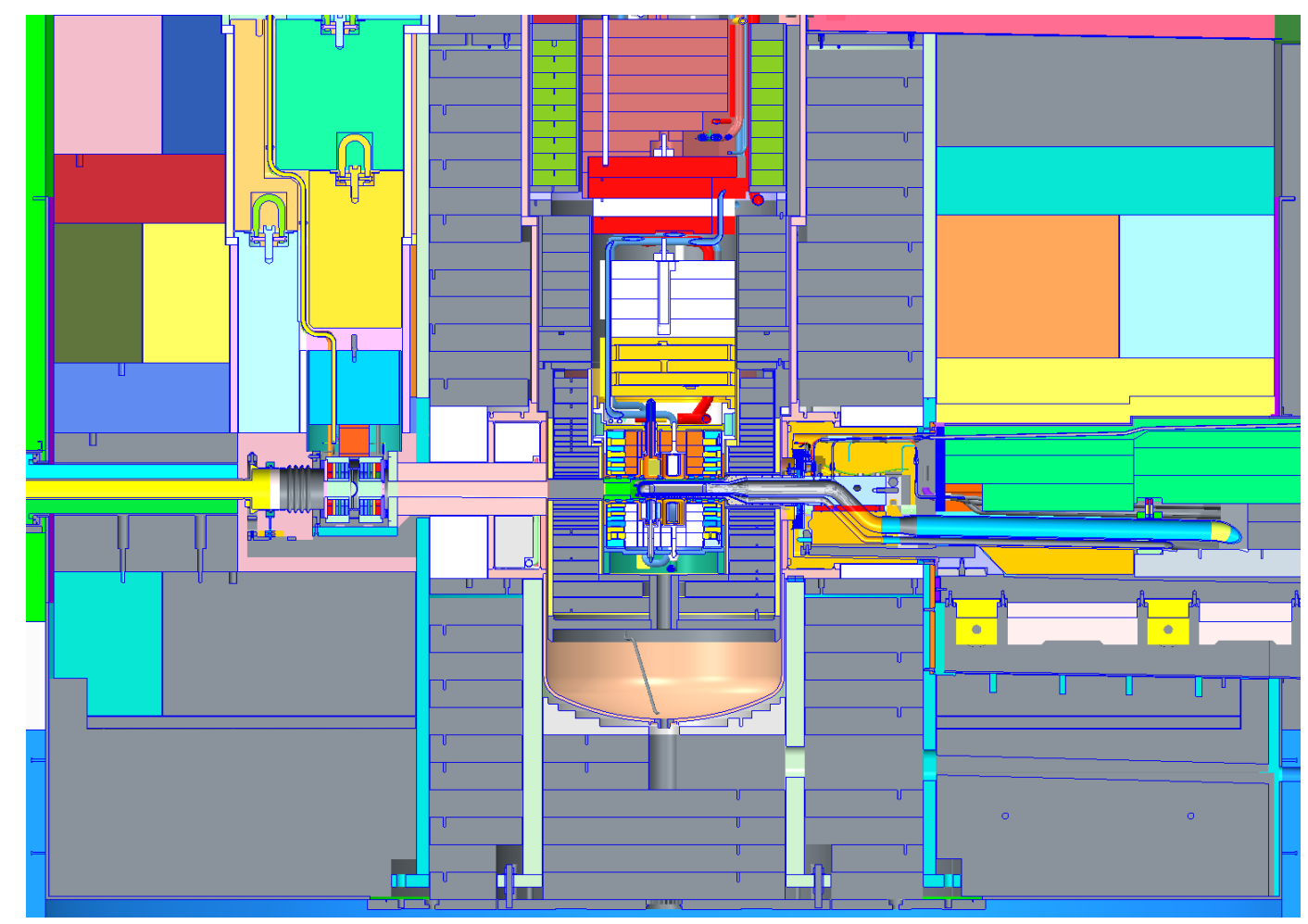

Figure 6.11. Monolith cross section showing vessel and monolith shielding systems. 

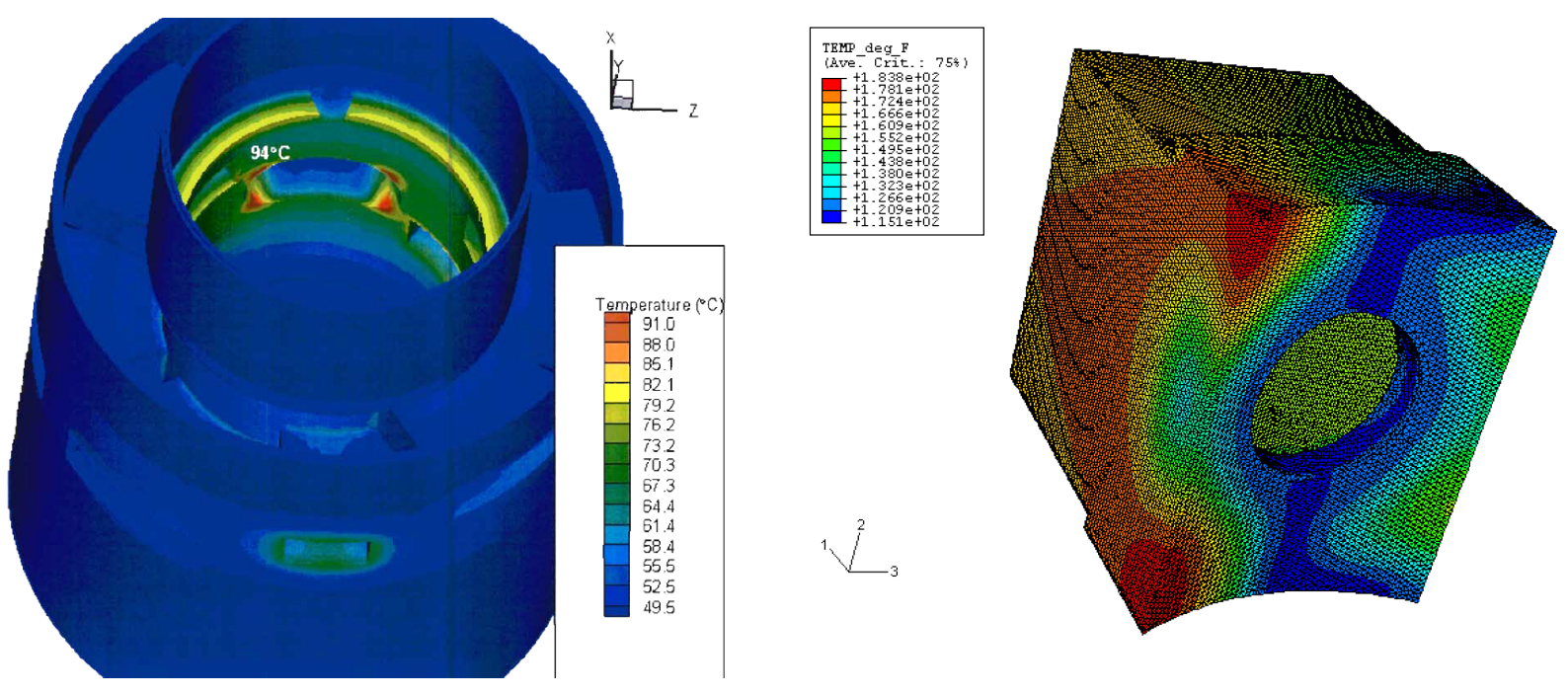

Figure 6.12. Temperatures in the ORP for the 1.0 GeV, 2.0 MW proton beam (left), and the temperatures in the core vessel target port for the $1.0 \mathrm{GeV}, 2.0 \mathrm{MW}$ proton beam (right).

If the analyses with $2.0 \mathrm{MW}$ of $1.3 \mathrm{GeV}$ proton beam operation show any deficiencies, further analysis will be performed to determine if operating parameters, such as coolant flow rates, can be modified to provide acceptable operating conditions.

Although currently seen as unlikely, any required hardware upgrades will be limited to the proton beam window and supporting utilities for reflector vessel and monolith shielding systems. Changes to related controls settings will be specified as needed. This work scope does not include neutron instrument core vessel inserts and their supporting utility systems, which are covered under FTS instrument systems (Section 6.3.6). Operational procedure changes will be implemented as necessary. New target utilities requirements will be coordinated and implemented.

\subsubsection{Target Utility Systems Evaluations and Upgrades}

This work scope is to ensure there are sufficient engineering, radiation, and operational margins in the FTS target utility systems for reliable operation with the PPU beam over the extended FTS lifetime and to install necessary upgrades. Coordination with other FTS systems is integral to assessing reliable system performance. Those systems will provide new utility requirements for PPU operation, if found.

Evaluations will consist of review of design basis documents (e.g., design and analysis calculations) and comparison with operational data from current operations as needed.

PPU target gas injection supply is part of the target utility upgrade scope. The actual required gas rate and pressure to the target will be determined by R\&D. The maximum envisioned gas rate is 20 standard liters per minute (SLPM), and the minimum is 4 SLPM. The maximum envisioned pressure is $100 \mathrm{psig}$. A recirculating gas compressor will be the primary means of gas supply to the target, with a once-through supply backup/supplement capability. Along with the 2.0 MW-capable target gas injection work scope, the specific performance requirements, installation, and safety approvals will be achieved for new gas injection supply equipment. PPU target gas utilities will build upon anticipated progress from the operations supported GI3 project [4].

Aside from new gas supply requirements for PPU target gas injection, modest upgrades are assumed in target utility systems; but this will be verified in an early project phase. Some operating adjustments to 
utilities may be needed. These may require updating design basis documents, piping and instrumentation diagrams, operating procedures, or training documentation.

For reference, the target utilities systems include

- $\quad$ Target primary water loops 1, 2, 3 and 4

$\circ$ Water loop 1-Mercury heat exchanger

$\circ \quad$ Water loop 2-Target water-cooled shroud and proton beam window

- Water loop 3-Moderators, shutters and core vessel inserts

$\circ \quad$ Water loop 4-Reflector plugs (heavy water)

- Secondary water loops

○ Deionized water loop

- Chilled deionized loop cooling

- Sensible chilled water loop

- Tower water

- Chilled water

- Helium gas distribution

- Nitrogen gas distribution

- Vacuum systems

Assumptions for target utility systems include

- Water loop 4 will have been converted to heavy water before the PPU.

- Hot off-gas recombination and recycle will be implemented for water loop 4 independent of the PPU.

\subsubsection{FTS Instrument Systems Evaluations}

This work scope ensures there are sufficient engineering, radiation, and operational margins in FTS neutron instrument systems with the PPU beam operation. No hardware upgrades are foreseen at this time, but this will be verified. Verification will result from a review of the design basis calculations, consideration of heating and radiation damage rates with PPU beam, and consideration of the extended 60 year lifetime. Changes to cooling might be necessary (e.g., to core vessel inserts in the downstream proton beam direction).

Twenty different design and analysis calculation (DAC) documents that establish the design basis for the most upstream components, mostly related to the core vessel inserts, have been identified for the FTS instrument suite. Each of these calculations will be reviewed to understand if the PPU beam to the FTS will result in a different conclusion from the one documented. Because these calculations have a degree of interconnectivity (i.e., the results of some calculations are the inputs to others), it is anticipated that the total number of documents reviewed will differ slightly from the set initially identified. Initial reviews will focus on the forward direction beam line, i.e., beam lines $8,9,10$, and 11. If calculated thermal or structural margins to limits are not sufficient for the PPU beam, current operational data may be reviewed to assess the level of conservativeness in the calculations.

Documents will need to be modified to recognize if the cited power inputs have changed as a result of the PPU beam to the FTS. At least a few of the documents will need to be recalculated to some extent, and it is possible that some number will require wholesale revision. 


\subsubsection{MOTS Upgrades}

PPU MOTS scope plans on the addition of a second carbon delay bed and related shielding to be installed in the GAR. The first one is being installed as part of the GI3 effort. High-rate target gas injection with the PPU will further add to the specific radioactivity and/or radioactive gas flow rate that eventually reaches MOTS. The second delay bed will ensure dose rates to workers and equipment in the MOTS equipment room and nearby areas will be maintained at acceptable levels. It also provides the ability to swap out one delay bed canister while maintaining MOTS operation.

Evaluations of the entire MOTS maximum flow capacity will be performed. The pressure profile up to 25 SLPM will be determined to establish a margin above the maximum envisioned steady 20 flow rate.

In addition to the delay bed, modifications to MOTS are planned, particularly in set points, indications, and controls. Further, as the primary concept for a recirculating gas compressor has the compressor unit(s) installed in the MOTS room, compressor procurement, installation, connections and interfaces to the equipment will be accommodated in a modified MOTS configuration.

In association with R\&D and GI3, questions regarding possible transient gas flow rates to MOTS are to be resolved and, as determined necessary, mitigated to maintain safe and reliable operation of MOTS.

Other upgrades to MOTS have been identified to improve operational availability. Two additional cryogenic carbon adsorber assemblies will be designed to improve the reliability of MOTS. One adsorber will replace the $\mathrm{LN}_{2}$-cooled cold trap, and the second one will serve as a spare. This flexibility is needed because of the long decay times required for carbon adsorber cold head maintenance and the more frequent maintenance expected with PPU high-flow gas injection.

This PPU FTS Systems work scope for MOTS includes the design, fabrication, installation, commissioning and documentation updates necessary to complete these goals to ensure safe and reliable operation with high-rate gas injection.

MOTS modifications are based on the following assumptions:

- Steady helium gas injection flow capability of up to 20 SLPM with PPU.

- The GI3 is complete with the following equipment designed, procured, fabricated, and installed:

- The first shielded carbon delay bed in MOTS for noble gas adsorption is installed in the GAR.

$\circ$ Piping and piping supports for the carbon delay bed will include connections for a second delay bed.

- A MOTS vent line GLS is in place to prevent mercury from entering the target service bay gas valve panel because of gas injection to the mercury process loop.

- A low-flow, once-through gas supply panel with the ability to monitor and control helium flow via EPICS is installed.

- Features to prevent mobilization of MOTS bed media by high-rate gas flows associated with expanding trapped gas bubbles (e.g., restricting orifice, surge suppression tank) are in place, or verification that mobilization is impossible under PPU steady or transient gas rates.

- The following documentation, procedures, and training materials will be updated:

- Design basis documents, including FSAD, Accelerator Safety Envelope (ASE), and Operational Envelope 
○ Operation Procedure Manual procedures

$\circ \quad$ Piping and Instrumentation Diagrams

- Target operation shift technical training materials and qualification standard

$\circ$ Remote handling procedures

\subsection{MW Target Module Design with High-Rate Gas Injection}

This work scope is to design, fabricate, and install a PPU beam-capable target module with gas injection, with a lifetime goal of at least 1,250 hours.

The target module consists of both the mercury vessel and the water-cooled bolt-on shroud. Most of the design and engineering effort lies in the mercury vessel. New jumpers to the carriage are planned to accommodate PPU gas injection; these are covered under the supporting hardware WBS sub-element.

Interfaces to the target carriage, core vessel, and reflector plugs will not change. Incorporation of swirltype small bubble generators and a protective gas wall are planned to reduce beam pulse pressure wave stresses and to reduce cavitation erosion.

The target modules at the SNS consist of an inner mercury vessel and an outer water-cooled shroud. The mercury vessel contains and directs the flow of the liquid mercury spallation target material. The region between the mercury vessel and the shroud is a stagnant, helium-filled space. The shroud serves to contain any mercury escaping from the inner mercury vessel. The target module is not a safety credited component. An exploded view of a target module is shown in Figure 6.13.

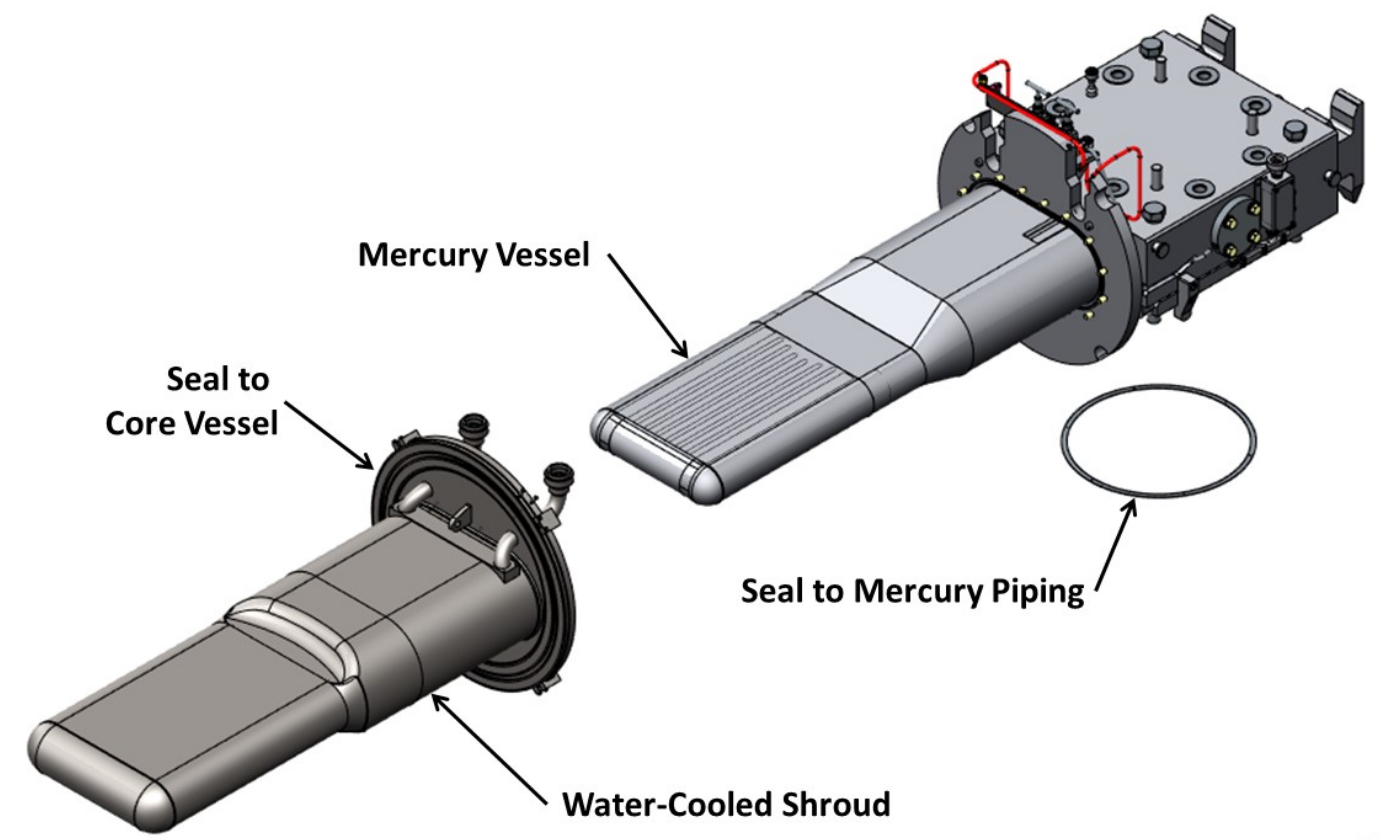

Figure 6.13. Exploded view of target showing outer water cooled shroud and inner mercury vessel.

When the proton beam strikes the target, energy is deposited in the mercury target material and the surrounding target module (Figure 6.14.) The short duration of the pulses causes short, high-intensity pressure pulses as the materials are heated. These pressure pulses introduce cyclic loads into the target 
module structure. In addition, the large pressures cause the mercury to cavitate, introducing complications to the loading evaluation and erosion of the target module structure.

In addition to high-frequency loading from pulses, the target modules are subject to lower-frequency loading cycles from changes in temperature brought on by heating from the proton beam. As the target receives energy, its structure and the mercury increase in temperature. When the beam trips or is otherwise stopped, the temperatures drop, and these changes introduce cycles of thermal stress.

The increase in beam energy and power in the PPU will increase the magnitudes of these loads over 1.4 MW and $1.0 \mathrm{GeV}$ values. The increased power will lead to stronger high-cycle pressure pulses, and the additional thermal energy will cause thermal stress cycles. In addition, the increase in beam energy from $1.0 \mathrm{GeV}$ to $1.3 \mathrm{GeV}$ will deposit energy deeper in the target module, changing the spatial loading of the target.

As mentioned in the system overview, the SNS target modules are first-of-a-kind technology. Although they have set records for performance, predicting and extending the service life of target modules remains an area of ongoing improvement. Although target modules have demonstrated the ability to perform at power levels up to $1.4 \mathrm{MW}, 7$ of the first 16 target modules developed mercury leaks out into the interstitial space between the mercury vessel and water shrouds. Such events are not a safety concern but they interrupt the user program and have a major impact on overall facility reliability.

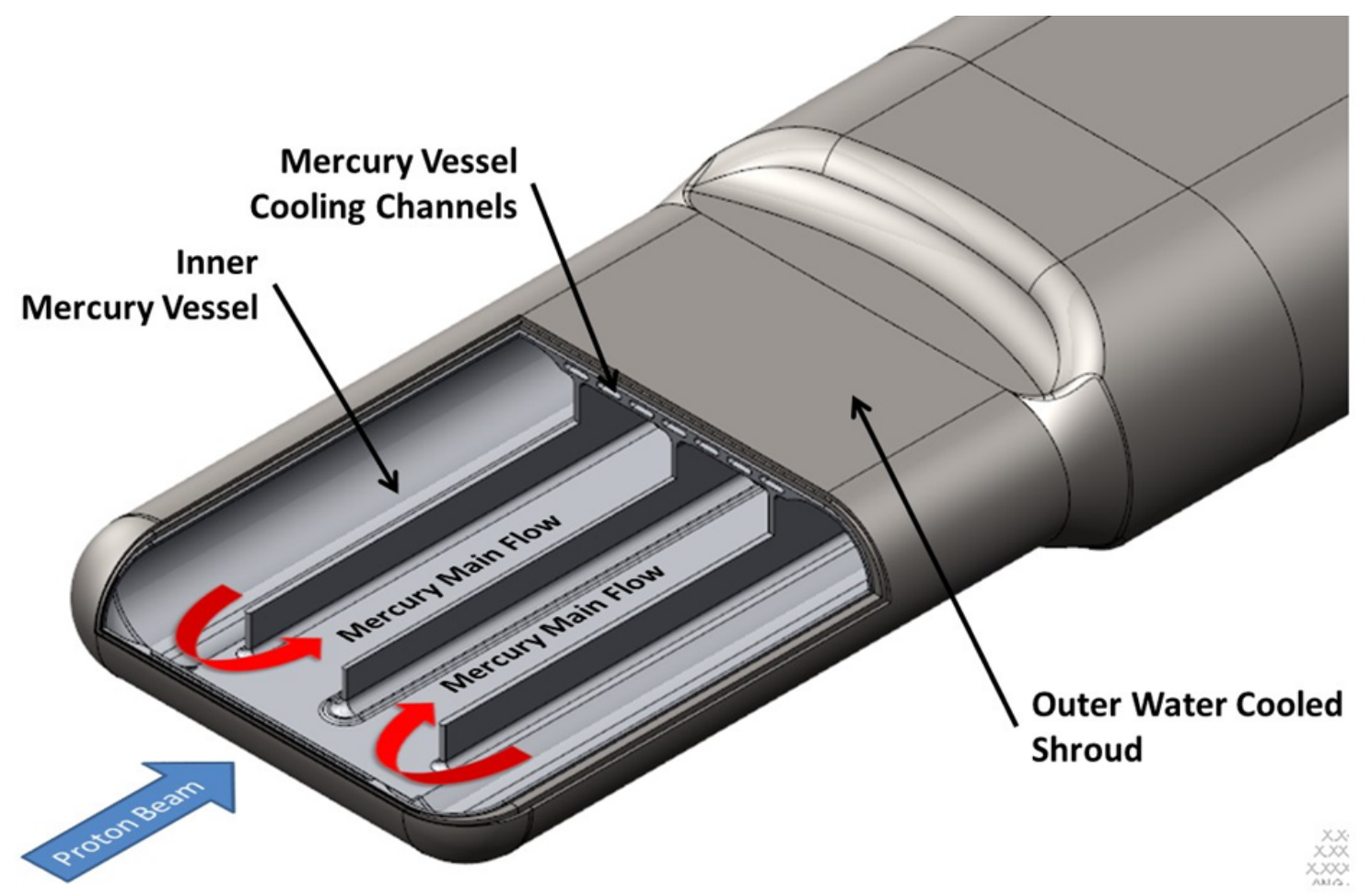

Figure 6.14. Current target mercury flow and structure.

Outside of PPU, SNS has a robust target improvement effort under way to improve target reliability at 1.4 MW and 1.0 GeV. This effort has already led to improved target designs and enhanced fabrication quality control. Additional knowledge gained from post-irradiation examination and target instrumentation, such as strain gauges, also continue to provide the information to improve these first-ofa-kind targets. This effort will continue, regardless of the PPU. This effort provides a strong base on 
which to build target modules that are reliable at $2.0 \mathrm{MW}$ and $1.3 \mathrm{GeV}$. This challenge is different from the current challenge for $1.4 \mathrm{MW}$ and $1.0 \mathrm{GeV}$ only by degree; therefore, the PPU will benefit from and expand on this current target improvement effort. However, the difference in degree is significant, with PPU representing an increase of over $40 \%$ in the energy delivered by each pulse into the target module. This will require additional improvements and systems beyond those required for reliable operation at 1.4 MW. Before discussing these needed improvements, however, it is prudent to review some of the basic constraints on the target module.

Current loadings and constraints of the target modules are documented in the target module design specification [13]. The target module is constrained by the geometry of the reflectors in the core vessel and existing hardware. The portion of the target forward of the seal to the core vessel protrudes into the core vessel, through an opening in the ORP and into a space in the IRP. (See Figure 6.15.) An assumption is that no significant geometry changes will be made to the IRP, so the target modules' geometry forward will continue to be subject to the same restraints. Similarly, it is assumed that the geometry of the target outside of the core vessel is constrained by the need to operate in conjunction with the existing target carriage and core vessel port. An assumption is that no changes will be made to the existing piping and connections of the target carriage. Because of radiation, contamination, and industrial hazards, installation and removal of targets is performed via remote handling, using master-slave manipulators and robotic servos. An assumption is that the current constraints, such as the need for remote friendly connections and features, will continue to apply to the PPU.

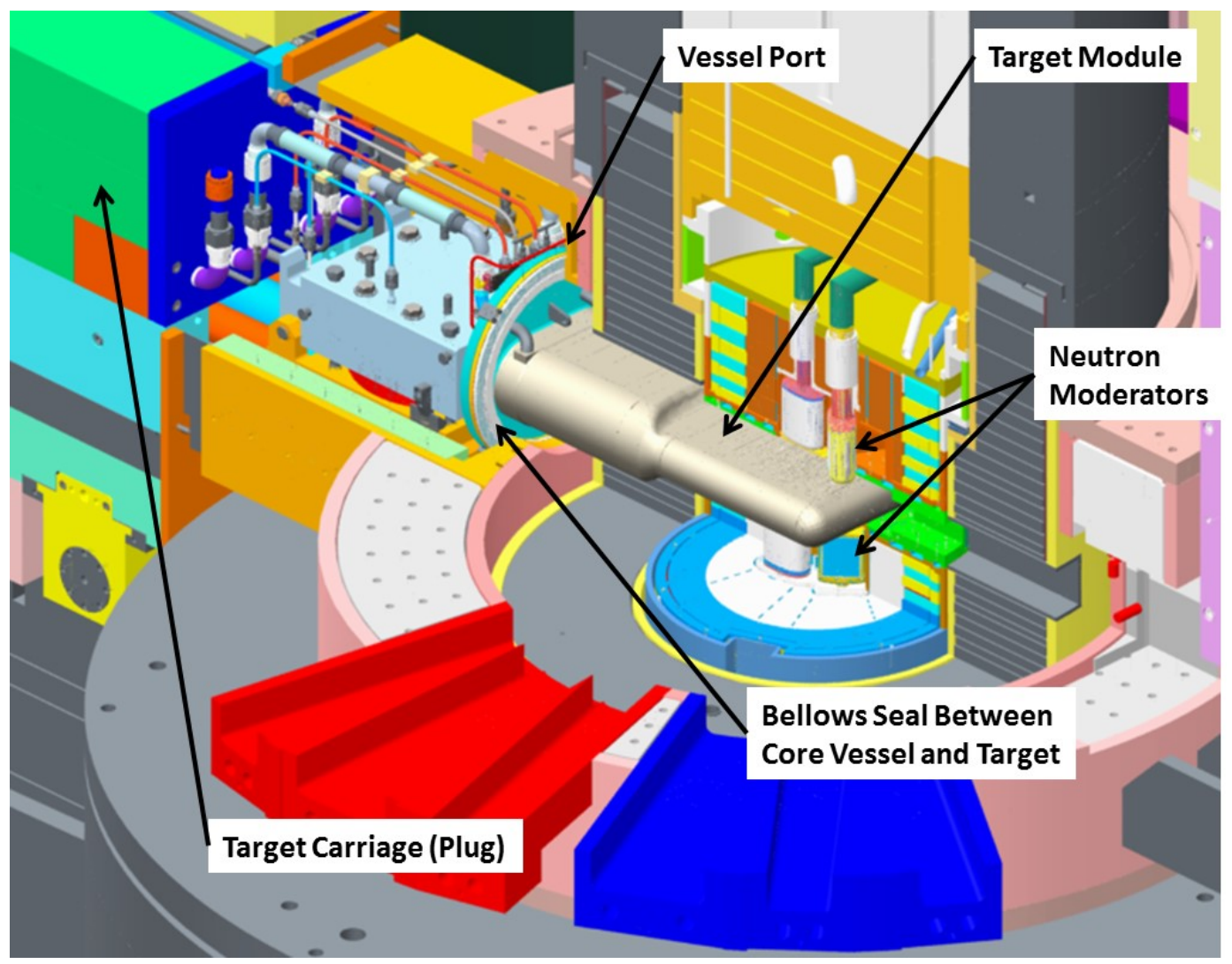

Figure 6.15. Cutaway showing target module in installed position.

Improvements are expected for both the target module mercury vessel and the water-cooled shroud. The changes needed to the water-cooled shrouds are expected to be minor compared with those to the mercury 
vessels. Initial review of the water shroud design margins indicates that any changes needed to the shroud will be minor and generally related to the modification of the existing internal cooling water flow passages of the shroud, which carry away the heat deposited into its structure by the particles and radiation. The current flow passages are positioned based on the energy deposition pattern at $1.0 \mathrm{GeV}$. At $1.3 \mathrm{GeV}$, the energy deposition pattern changes, which should be attributed to changes in the internal flow passages. Although more significant changes to the water shroud cannot be ruled out because of changes to the internal mercury vessel, these changes are expected to be minor because of the external geometry constraints.

The design challenges associated with the mercury vessels are much more significant. The development of target mercury vessels that will be reliable at $2.0 \mathrm{MW}$ and $1.3 \mathrm{GeV}$ has two main thrusts. First, the ability of the target module to withstand the pulse and thermal stress loadings will be improved. Second, new features to reduce these loadings and pulse-induced cavitation damage will be developed and implemented.

The target mercury vessel will be improved by changes in its geometry and construction to better withstand loadings and damage. This has been and continues to be part of the target reliability improvement efforts for reliable 1.4 MW and 1.0 GeV operation. Changes including welding and geometry modifications to increase the fatigue resistance of the structure have been incrementally implemented. In addition, minor changes to the internal mercury flow have been explored and have proved capable of significantly reducing cavitation damage erosion in the case of the jet-flow target. (See Figure 6.16). As part of the PPU effort, design improvements and optimization will be aggressively pursued to maximize the fatigue resistance of the target module structure. This includes not only evolutionary improvements, such as those that have been pursued to date, but also wholesale modification and optimization of the target mercury vessel's internal geometry. This is expected to include alterations such as modifications in the thicknesses and positions of features; but other concepts, such as very different mercury flow patterns, will also be evaluated. It is assumed that meeting this challenge is possible with the current $316 \mathrm{~L}$ stainless steel material, so no significant effort to qualify a new structural material is included in the PPU effort.
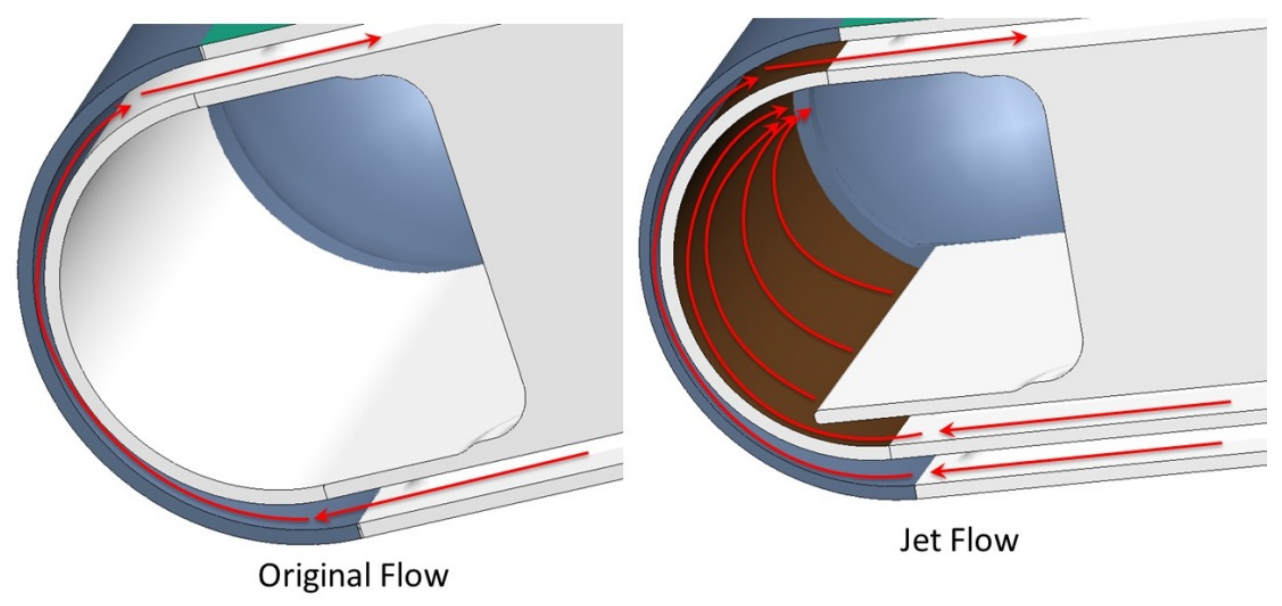

Figure 6.16. Cutaway view of nose of target mercury vessel showing the original mercury flow and the jet flow improvement that reduced cavitation damage.

To reduce the loadings and damage on the target modules, gas injection into the flowing mercury will be key. The addition of noncondensable gas into the flowing mercury has been shown to reduce structural loading of the target structure. The gas acts to cushion and dissipate the energy deposited by the proton 
beam. However, the efficacy of this mechanism is impacted by several variables, including the size and spatial distribution of helium gas bubbles. In addition, other uses of gas, such as creating gas layers at the walls of the structure, have been shown to be highly effective at reducing or eliminating erosion from mercury cavitation [1]. The current target improvement plans for 1.4 MW include the implementation of gas injection into the mercury at low-flow rates. This effort is a key step toward understanding the behavior of a two-phase mercury/helium mixture; but higher gas flow rates, better bubble generation technology, and the implementation of protective gas walls also are expected to be necessary to the development of target modules that will provide long, reliable performance at $2.0 \mathrm{MW}$ and $1.3 \mathrm{GeV}$. PPU includes R\&D scope to develop high-efficiency bubble generator requirements and specifications for the $2 \mathrm{MW}$ target design. R\&D will further define the requirements for the target gas-wall configuration.

A conceptual design model of the forward portion of the target is shown in Figure 6.17. It features a tapered forward region to direct and accelerate beam window corner flow and to reduce forward mass, thus raising the frequency of a pulse-driven dilatational mode sufficiently above $60 \mathrm{~Hz}$. Swirl-type small gas bubble generators are positioned forward in the bulk inlets to reduce the bubble coalescence potential. The design features a jet-flow covering the inside of the inner beam window and a gas wall at the central half of the same surface.
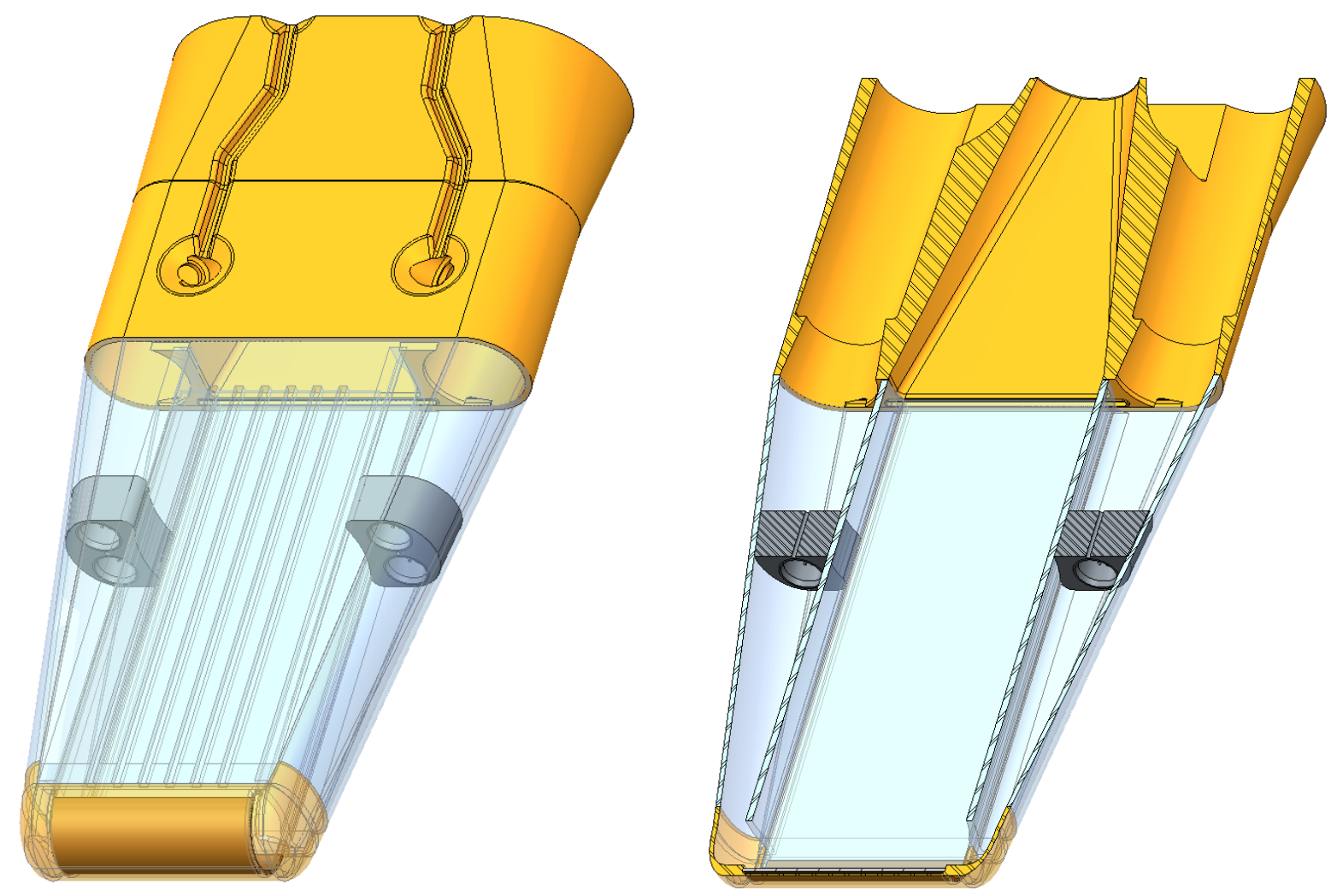

Figure 6.17. Conceptual design of the forward and transition sections of a 2 MW PPU target.

The effects of gas injection on other systems outside the target module must be managed and have been addressed in other sections of this report.

In summary, mercury target modules are a key technology in neutron production at the SNS. To date, the target modules have successfully operated at high power levels but have had a negative impact on reliable, high-power operation critical to the user program. Past research and current efforts provide an 
excellent base from which to build target modules that will reliably operate at $2.0 \mathrm{MW}$ and $1.3 \mathrm{GeV}$. This will require design improvements and optimization of the internal structure of the target modules, as well as the implementation of advanced gas injection systems to reduce loading and cavitation damage to the target.

Mercury target design will be based on the following assumptions:

- Current efforts associated with 1.4 MW target reliability improvements - including target postirradiation examination, instrumentation, and low-gas injection flow rates-will continue.

- $\quad$ PPU R\&D will define the requirements and specifications for the target bubblers and gas wall.

- Geometry constraints on the target will remain the same.

- No changes will be made to the existing target carriage or core vessel port-target interfaces.

- Target installation and removal will be performed via remote means.

- The target structural fabrication material will remain 316L stainless steel in various forms.

\subsubsection{Safety, Controls, and Operations}

These crosscutting activities are essential to operational readiness for PPU beam on the FTS. Four of the five unreviewed safety issues identified in the PPU Preliminary Hazard Analysis [14] fall within FTS Systems:

- Increased beam particle energy from $1 \mathrm{GeV}$ to $1.3 \mathrm{GeV}$ - effect on target spallation product inventory

- Increased beam particle energy from $1 \mathrm{GeV}$ to $1.3 \mathrm{GeV}$ - effect on target core vessel component heat distribution

- Injection of helium into the circulating target mercury in the target module to control the rate of cavitation erosion of the target module

- Increased hydrogen inventory of the target cryogenic moderator system due to the proposed installation of a catalytic conversion stage to convert ortho-hydrogen into para-hydrogen.

Hazards associated with target gas injection (third bullet) require an integrated systems approach to developing designs, controls, and operating procedures that sufficiently mitigate accident risks and for which safety authorization must be obtained. Relevant systems include mercury process systems, target utility systems, MOTS, and the $2 \mathrm{MW}$ target. Their controls for gas injection are in addition to existing control functions. Ensuring safe operation of gas injection must consider interactions between new and existing controls. If necessary, new credited engineering controls may be required. Progress on safety authorization for the GI3 effort will provide a foundation for PPU gas injection safety.

Safety authorization begins in the design process and culminates with a positive outcome from an Accelerator Readiness Review and authorization from the DOE Field Office. Several crucial steps are to be completed in between, for example updates to the Facility Safety Assessment Document and Accelerator Safety Envelope. These require coordination, interaction, and iteration with PPU Control Account Managers and operations staff; responsibility for this coordination falls within this work scope area. 
Controls touch on nearly all of the FTS Systems project scope. Much of the controls work will be gas injection related, but other changes are envisioned. For example, in target utilities, controls to address needed changes to cooling flows are planned. Again, this work scope performs a systems integration function between the technical systems and controls.

Similarly, operating procedures and required training updates will be made ready for new and modified systems installed for PPU. This work scope will ensure that operator training for these systems is prepared. The procedures and training will be needed for operation authorization.

\subsection{REFERENCES}

[1] B. Riemer, Conceptual Design Report: Mercury Target Gas Injection, SNS 106010000-TR0132, R00, Oak Ridge National Laboratory, 2016.

[2] B. Riemer, PPU FTS Systems 2 MW Target Development Plan, PPU-P05-PN0001, R00, Oak Ridge National Laboratory, April 2017

[3] Target Management Plan, 106010000-PN0005, R00, Oak Ridge National Laboratory, March 2017

[4] P. Rosenblad, Gas Injection Initial Implementation (GI3) Conceptual Design Review (CDR) Report, 106010000-DE0004, R00, Oak Ridge National Laboratory, September 2016.

[5] K. Okita, S. Takagi, and Y. Matsumoto, "Propagation of pressure waves, caused by a thermal shock, in liquid metals containing gas bubbles," Journal of Fluid Science and Technology 3(1), 116-128, (2008).

[6] D. Hilscher, F. Goldenbaum, U. Jahnke, et al., "Neutron multiplicity distributions for GeV proton induced spallation reactions on thin and thick targets of $\mathrm{Pb}$ and U," p. 176 in Proc. of Int. Workshop on Nuclear Methods for Transmutation of Nuclear Waste, eds. M. Khankhasayev, H. S. Plendl, and Z. Kurmanov, Dubna, Russia, May 29-31, 1996, World Scientific, Singapore, 1997.

[7] F. X. Gallmeier, Neutronics Impact of the SNS Proton Energy Upgrade from 1.0 to 1.3 GeV, SNS106100200-TR0186-R00, Oak Ridge National Laboratory, 2011.

[8] W. Lu et al., Impacts of $1.3 \mathrm{GeV}$ Proton Beam on the Energy Deposition of FTS, SNS-106100200TR0218-R00, Oak Ridge National Laboratory, 2016.

[9] A. Ibrahim and K. Bekar, Design Analyses of SNS Target Station Biological Shielding Monolith with Proton Power Upgrade, SNS-106100200-TR0219, Oak Ridge National Laboratory, 2016.

[10] I. I. Popova, Radionuclide Inventory for Mercury in the SNS Target Loop for PPU Conditions, 2 MW, SNS-106100200-DA007-R00, Oak Ridge National Laboratory, 2016.

[11] W. Lu, Moderator Performance of the SNS As-built Configuration, SNS-106100200-TR0199-R00, Oak Ridge National Laboratory, 2013.

[12] C. Barbier, D. Felde, R. Sangrey, et al., TTF Gas Holdup Experiments, SNS 106010101-TR0019 R00, Oak Ridge National Laboratory, January 2016.

[13] D. Winder, Target Systems, Target Module Design Specification, SNS 106010101-TS0001, Oak Ridge National Laboratory, 2016. 
[14] R.M. Harrington and S.M. Trotter, Preliminary Hazard Analysis in Support of the Proton Power Upgrade Project, PPU-01-ES0001-R00, Oak Ridge National Laboratory, January 2017. 


\section{CONVENTIONAL FACILITIES}

The CF WBS element provides the new buildings and associated utilities to support the technical equipment required for the PPU project. There are two major scope elements for CF: the klystron gallery and the RTBT stub. An additional scope element is the EPICS controls for the new klystron gallery heating, ventilation, and air-conditioning (HVAC) unit.

\subsection{KLYSTRON GALLERY}

The CF scope of work for the PPU in the klystron gallery area consists of new construction and existing facility modifications. The RF and cryo level 2 WBS elements install the technical equipment in the klystron gallery and the linac, which is required to increase the proton beam energy to $1.3 \mathrm{GeV}$. The CF provides the new building and utility distribution needed for the technical equipment. CF also provides the EPICS controls for the new HVAC unit. See Figure 7.1 for a high-level view of the klystron gallery project area.

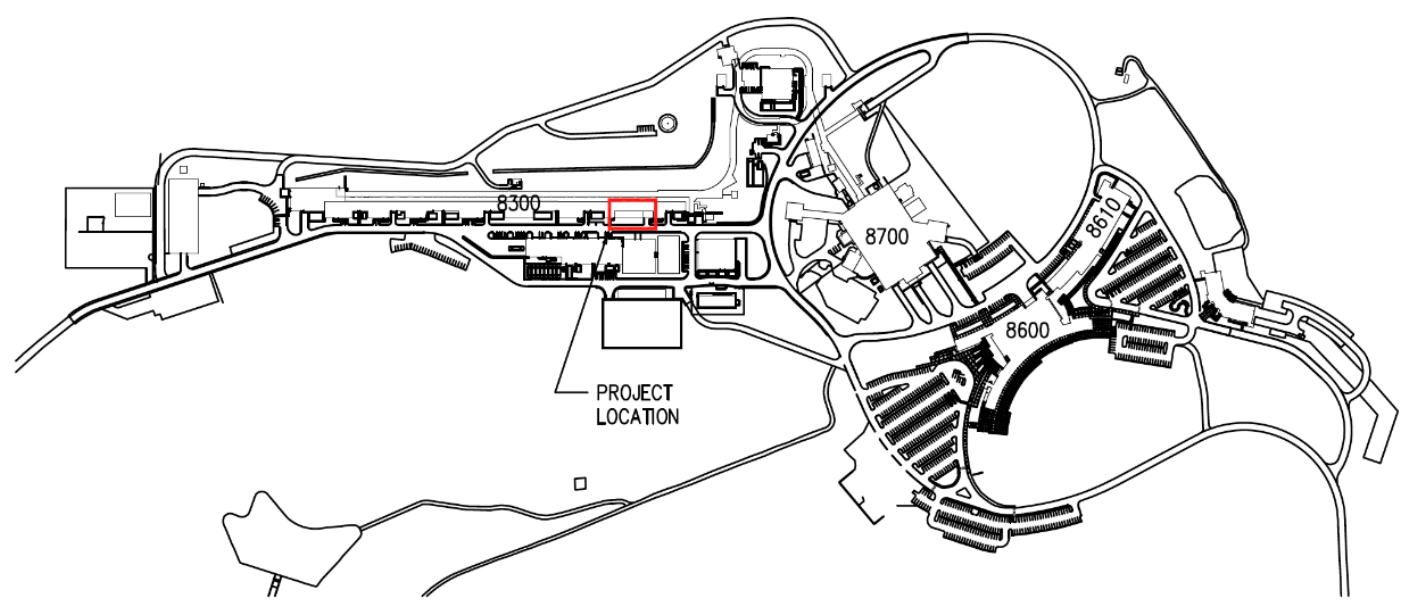

SNS CAMPUS MAP

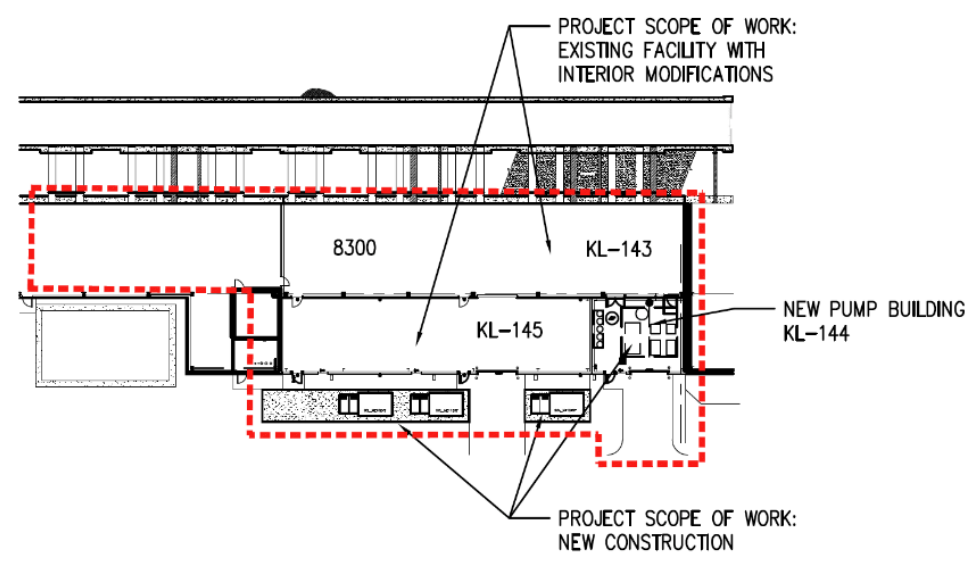

PROJECT SITE MAP

Figure 7.1. SNS campus map with detailed insert of the klystron gallery conventional facility activity. 


\subsubsection{Klystron Gallery New Construction}

\subsubsection{New pump building}

A new pump building (KL-144) will be constructed to house the new deionized water cooling loop for the accelerator equipment. The approximately 25 by 25 foot building will be between the east end of the existing klystron gallery buildout (KL-145) and the west end of the HEBT service building (8340).

\section{Structural Systems and Site Improvements}

The new pump building (KL-144) will be a steel frame structure with a metal deck roof (IBC Type II construction). The foundation will be a reinforced concrete slab with thickened edges (no isolated spread footings). The structure will be designed to meet SDC-2 requirements for wind and seismic activity. In addition to a $20 \mathrm{psf}$ live load (LL), the roof will be designed for a $50 \mathrm{psf}$ collateral load and a suspended load of 3,000 lb (tank). The floor slab will be designed for an LL of $500 \mathrm{psf}$ and will have floor drains and a sump.

Two concrete pads will be installed within the room for the deionized water pump/motor skids.

A new driveway will be installed from Los Alamos Drive to a rollup door in the pump building. A sidewalk will also be connected to the new man door.

\section{Building Enclosure Systems}

The pump room building will be faced in a flat-face insulated metal panel to match other sections of the klystron gallery buildout. Special details will be required for it to interface with the deep rib panel of the HEBT service building. Matching louvers will be provided as required to serve mechanical equipment.

The roof system will include a corrugated metal deck with insulation board and a built-up roof to match the existing roof. The roof system will be a Factory Mutual Global Class 1-approved type and will meet UL Class A fire exposure requirements. Gutters and downspouts will provide drainage from the roof. Expansion joints will be provided between the new pump building and adjacent buildings.

New double doors will be cut into the south wall of the klystron gallery (KL-143) to provide interior access into the new pump building (KL-144). The existing single man door will be removed and infilled with panels to match the existing door. A 3 by 7 foot man door in the south wall of the pump building will provide access to the building and egress to the exterior. A 12 by 12 foot overhead door will also be provided in this wall.

The concrete slab-on-grade will receive an epoxy sealer for dust control and durability. There will be a floor drain and a sump within the room.

\section{Life Safety Requirements}

The new pump building (KL-144) will be separated from the HEBT service building (HS-102) by a ULlisted 2 hour partition. Egress will be provided directly to the outside via the man door in the south wall in compliance with National Fire Protection Association (NFPA) 101, Life Safety Code. Access to this exit from the klystron gallery (KL-143) through the new pump building (KL-144) will be provided. 


\section{Mechanical Systems}

The new pump building (KL-144) will be protected from freezing by a unit heater and ventilated by an exhaust fan.

Tower water (supply and return) lines will be provided to cool the new DI pump skid. The tower water lines will be routed from the adjacent HEBT service building (8340).

A process waste sump and associated pumps and piping will be installed to dispose of liquids from floor drains and other influent sources.

\section{Fire Protection Systems}

\section{Sprinklers}

Piping will be extended from the existing klystron gallery buildout (KL-145) and new sprinklers provided in the new pump building (KL-144). The occupancy hazard classification is Ordinary Hazard Group 2 ( 0.20 gpm over 3,000 square feet), and the sprinkler system design will comply with NFPA 13, Standard for the Installation of Sprinkler Systems. Seismic bracing for sprinklers will be provided. Installation of seismic bracing will be in accordance with NFPA 13.

\section{Fire Alarm}

A very early smoke detection apparatus (VESDA) system will be extended into the new pump building (KL-144) from the existing klystron gallery buildout (KL-145) building. The fire alarm notification circuit in the existing klystron gallery buildout (KL-145) building will be extended into the new pump building (KL-144). A manual pull station will be provided at the new pump building (KL-144) exit door.

\section{Electrical Systems}

Power, lighting, and communications will be provided for the new pump building (KL-144). Circuits will be routed from panels/racks in the existing klystron gallery. Emergency light units and exit signs for the new pump building (KL-144) will be routed from existing emergency power panels in the klystron gallery. Light fixtures and exit signs will use LED type illumination. Lightning protection will be extended to the new pump building (KL-144) from the existing klystron gallery system.

\subsubsection{New Electrical Distribution}

The addition of new high-power RF equipment and three high-voltage converter modulators (HVCMs) and operation of the HEBT-ring-RTBT magnets at higher current will significantly increase the SNS site electrical load. To support new klystron gallery electrical loads, concrete pads for three new $1500 \mathrm{kVA}$ transformers (supplied by the RF group) will be installed just south of the gallery buildout (KL-145). These new transformers will support the new HVCMs on a one-to-one basis. A fourth concrete pad and associated conduit stub-outs will also be installed for future equipment.

Three new $13.8 \mathrm{kV}$ interrupter switches will be installed (one on each pad) to isolate the modulator power supplies from the electrical distribution system.

Underground duct banks will be provided from existing manhole M20 to the new pad-mounted interrupter switches. A new $13.8 \mathrm{kV}$ feeder will be spliced into existing feeder FDR-3014 in manhole M20. The CF scope for the new electrical distribution ends at the primary of the interrupter switch. The remaining electrical distribution is part of the RF scope. The switches will be closely coupled with 
cabling/busses to the transformers. Secondary cabling from the transformers will be fed through underground conduits into the existing klystron gallery buildout (KL-145).

\subsubsection{Klystron Gallery Existing Facility Modifications}

Modifications in the east end of the existing klystron gallery are required to support the new accelerator equipment. The CF utilities (mechanical, electrical, fire protection) will be installed in the klystron gallery after the existing shop and storage areas are relocated.

\subsubsection{Mechanical systems}

The section of the klystron gallery with new accelerator equipment will be heated and cooled with a rooftop-mounted chilled and heated water HVAC unit. The air will be strategically distributed to technical equipment loads by overhead galvanized ductwork. The cooling capacity of the HVAC unit was selected to be 85 tons and $30,000 \mathrm{cfm}$.

A pipe rack will be installed in the klystron gallery to support the following piping utilities:

Chilled water will be routed to the new HVAC unit from the HEBT service building (8340).

Heating water lines will be routed to the new HVAC unit from existing piping located nearby in the klystron gallery.

Compressed air lines for utility air, like the existing systems in the klystron gallery, will be routed to various locations for valve actuation, tool use, and other common uses.

Process water lines for makeup water will be routed to technical equipment, like the arrangement for existing systems in the klystron gallery.

The main deionized water supply and return headers in the klystron gallery will be routed from the new pump building to conveniently located valves for technical equipment connections. (The deionized water system is part of the RF-Water Utilities scope; however, the piping will be installed within the CF scope for ease of construction).

Condensate from the rooftop HVAC unit will be routed to the storm drain.

\subsubsection{Fire protection systems}

\section{Sprinklers}

If any changes to the existing sprinkler system in the east end of the klystron gallery (KL-143) are necessary to coordinate the sprinkler piping with the other utilities and technical equipment installations, the sprinkler system will be modified as necessary to provide coverage in compliance with NFPA 13, Standard for the Installation of Sprinkler Systems.

\section{Fire Alarm}

VESDA smoke detection will be extended into the east end of the klystron gallery (KL-143) building from the existing system to the west of the klystron gallery buildout (KL-145) building. Existing spot smoke detectors in the klystron gallery (KL-143) will be removed. Area notification appliances will be relocated as required for proper coverage requirements. 


\subsubsection{Electrical systems}

Existing power distribution panels in the klystron gallery (KL-143) will supply power to new panels for technical equipment supporting systems, e.g., vacuum, controls, diagnostics, cooling loops.

Power will be provided for the new HVAC unit to be installed on the roof of the klystron gallery (KL-143).

New cable trays will be installed to distribute the power and communications cabling throughout the klystron gallery (KL-143) and buildout area (KL-145).

Existing lighting fixtures in the klystron gallery will be removed and new LED fixtures installed to accommodate the new technical equipment.

Communications for voice and data will be provided to convenient locations for phones and network connections.

\subsubsection{Controls}

The new HVAC unit with associated piping systems instrumentation will require new controls. These controls will follow the existing model for SNS CF controls, which are implemented using SNS standard Allen Bradley PLCs and I/O modules integrated with the SNS EPICS system. The SNS Controls Group will develop the required controls design, drawings, sequences, ladder logic, test plan, and EPICS IOC database. Operator interfaces, alarms, and archived data for this system will be configured using SNS standard EPICS tools.

\subsection{RTBT STUB}

The RTBT stub will be an extension of the existing RTBT tunnel, which houses the proton beam that serves the FTS. The stub is to be located at the juncture where the proton beam will be split when the STS is built, forming a second proton beam line directed to the STS through the future ring-to-target-two (R2T2) tunnel. The RTBT stub is to be constructed during an extended shutdown of the SNS required during the PPU to upgrade the ring injection area. Other concurrent shutdown-related activities include an inner reflector plug change, cryogenic moderator upgrades, cryomodule installation, and related RF installation and startup tasks. This scope is included in the PPU to enable construction of the STS, including the R2T2 tunnel, without requiring an additional prolonged shutdown of the FTS during the STS construction period. This strategy should reduce the overall impact on science from the PPU and STS projects. See Figures 7.2-7.5 for an overview of the RTBT stub configuration and location.

Construction of the RTBT stub is currently scheduled to begin in the late spring of 2023, coinciding with a planned extended shutdown of the proton beam line and, typically, drier weather conditions. Construction of the RTBT stub within a 5 to 6 month period is considered feasible; however, some portions of the stub project will be constructed either before or after the beam shutdown period.

The RTBT stub length selection was based on a study that considered key design requirements. The important considerations included providing sufficient shielding, facilitating future continuation of the tunnel construction, allowing reasonable site slopes, accommodating revised storm drainage, and minimizing constraints on the future STS site plan. 


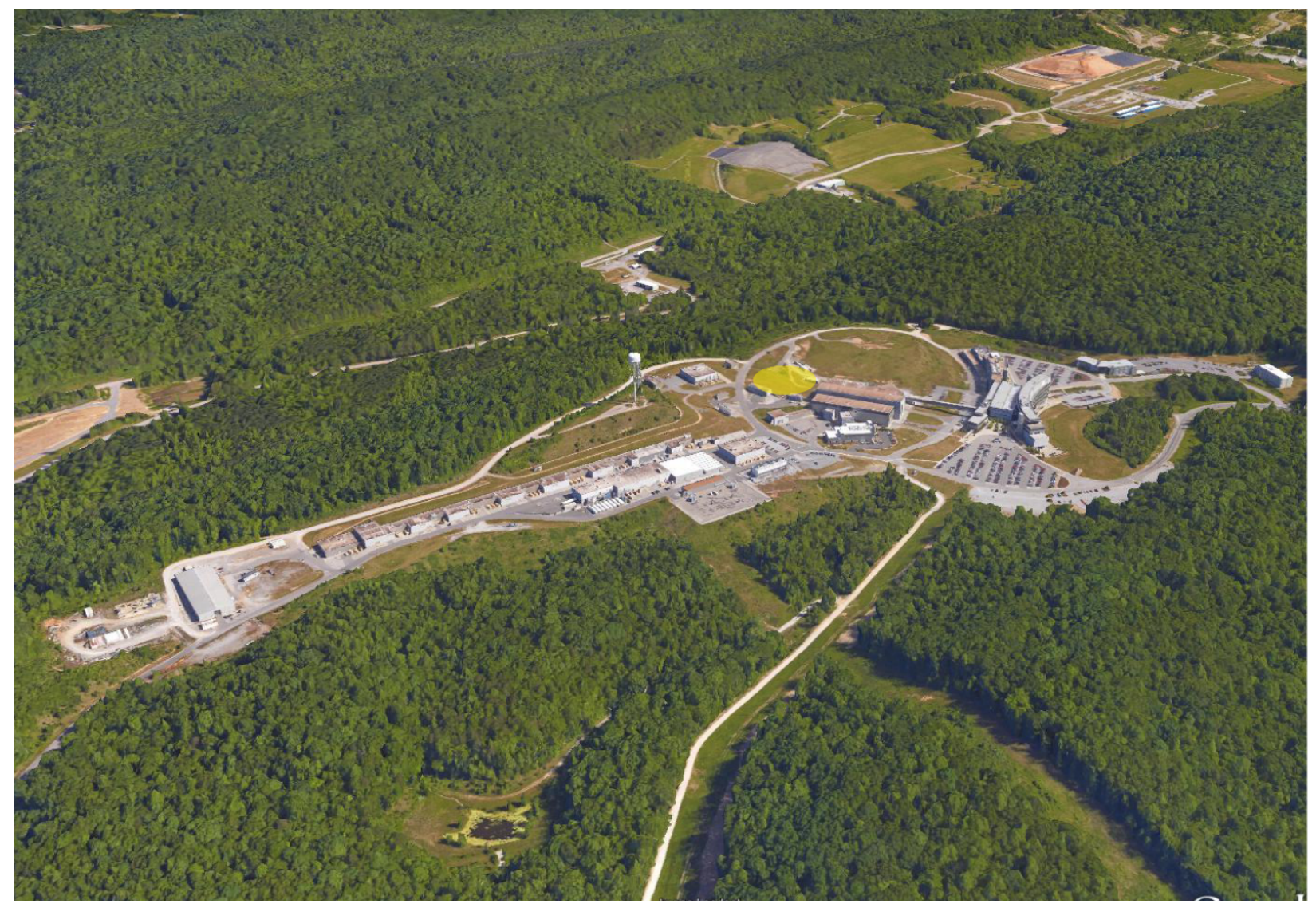

Figure 7.2. Aerial view of the Spallation Neutron Source campus. Yellow highlighting indicates the project area.

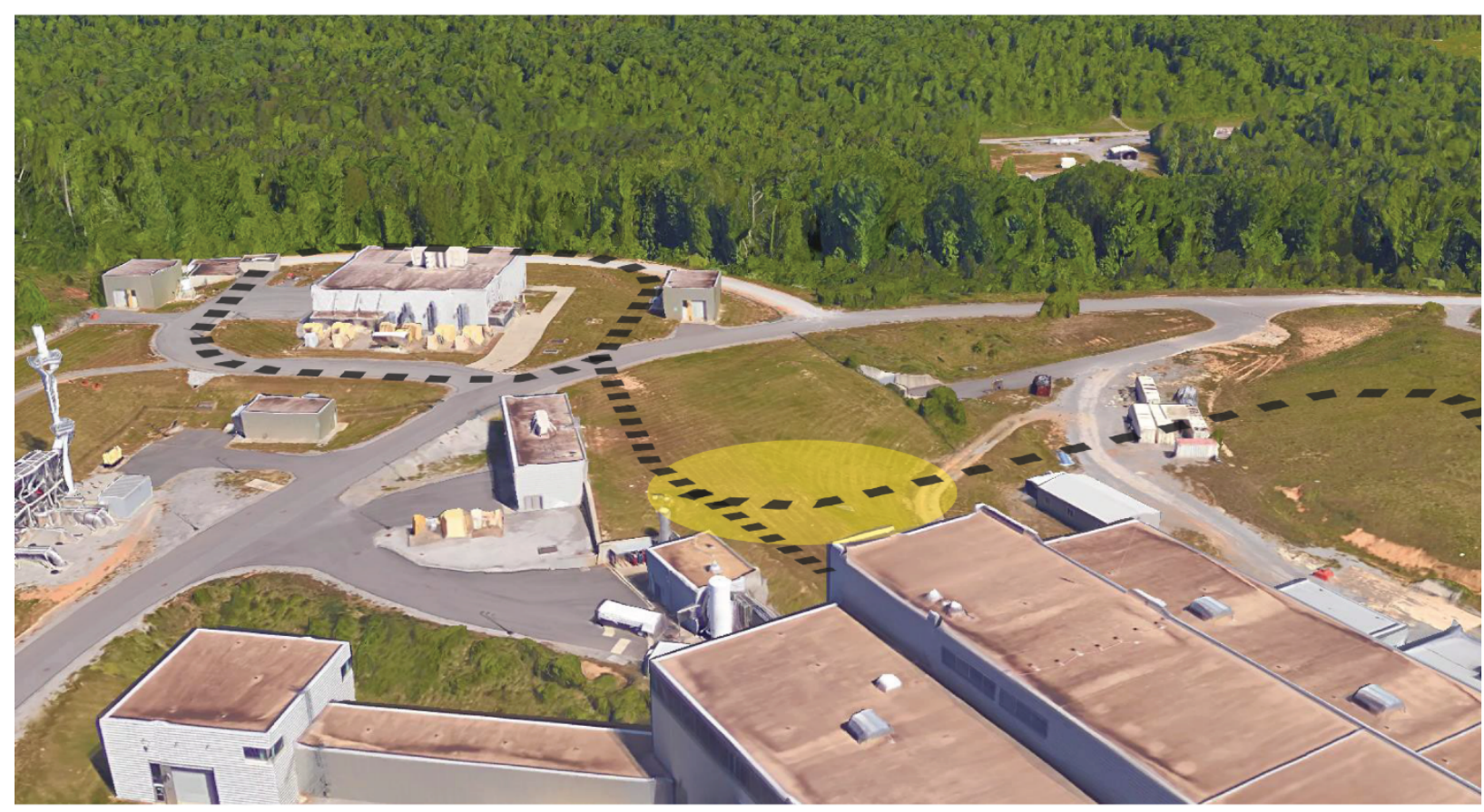

Figure 7.3. Aerial view of SNS. The RTBT stub project location is highlighted in yellow. 


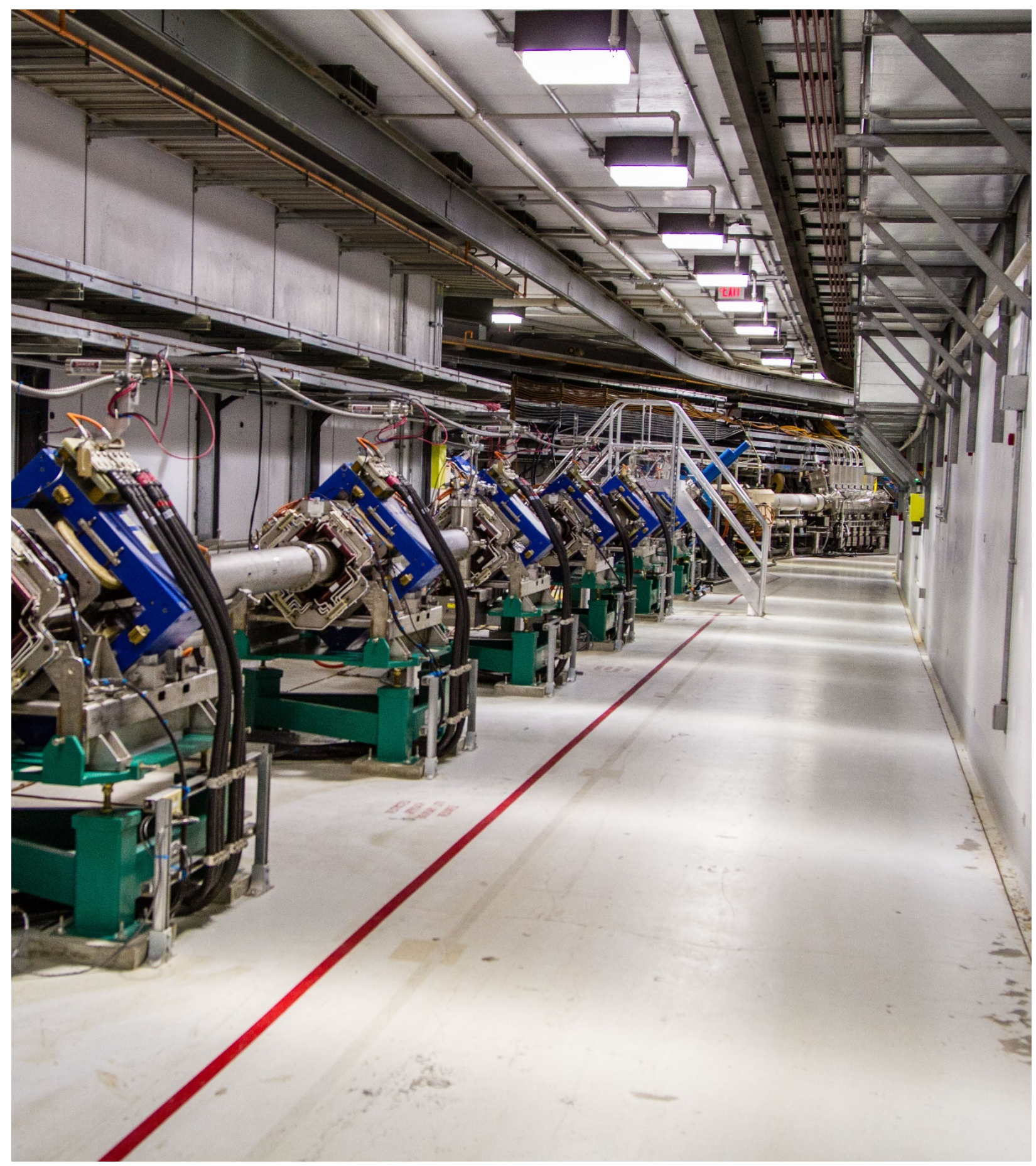

Figure 7.4. Existing RTBT tunnel looking toward the ring. The existing proton beam line is on the left; the stub will connect to the right side of the tunnel. 


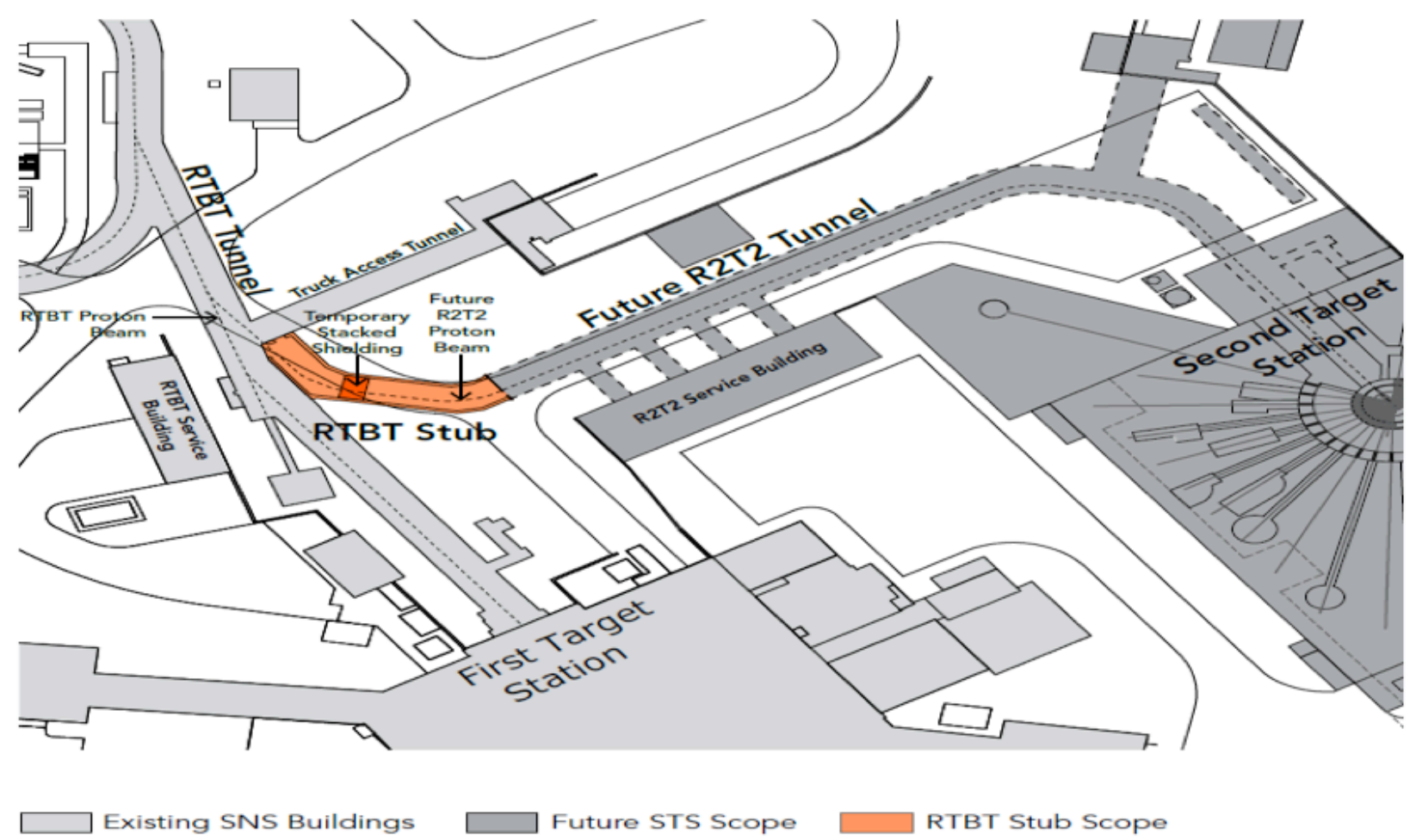

Figure 7.5. Overall RTBT stub configuration.

\subsubsection{Shielding Requirements}

Shielding calculations, performed as part of part of the SNS project, show that 18 feet and 6 inches of concrete and soil shielding is required from the inside of the RTBT tunnel concrete to the ground surface. This material is necessary to provide sufficient shielding to meet DOE requirements for radiation exposure to personnel on the berm during high-beam-loss conditions and during a postulated beam spill accident. The same amount of shielding is planned for this project.

A temporary shield wall and associated PPS equipment will be provided by the ring WBS to allow entry into the RTBT stub downstream of the shielding during FTS operation. The interlaced shielding blocks, totaling 3 meters thick, will be located as close to the RTBT tunnel as possible while maintaining sufficient earth shielding between the RTBT tunnel and the RTBT stub. The temporary shielding will be removed following construction of the R2T2 tunnel and the installation of most of the STS proton beam line.

\subsubsection{Civil/Geotechnical}

The geotechnical and structural stability of the existing RTBT tunnel, proposed RTBT stub, and future R2T2 tunnel are critically important design concerns closely related to the alignment and performance of the proton beam. The existing earthen berm that covers the RTBT tunnel is around 16 feet thick.

Preliminary analysis indicates that, if a berm thickness of 7 feet is maintained during the construction of the stub, the structural integrity of the tunnel will be maintained. That distance will also minimize the 
amount of RTBT tunnel rebound from removal of the earthen berm. It is expected the rebound will be elastic, and the RTBT tunnel will return to its former elevation after construction is complete.

Site grading associated with this project will require that existing Building 8940 (Carpenter and Paint Shop) be demolished.

Figure 7.6 shows an overview of the site after final grading and installation of new stormwater piping and shows existing underground utilities. Figure 7.7 indicates the present elevation configuration.

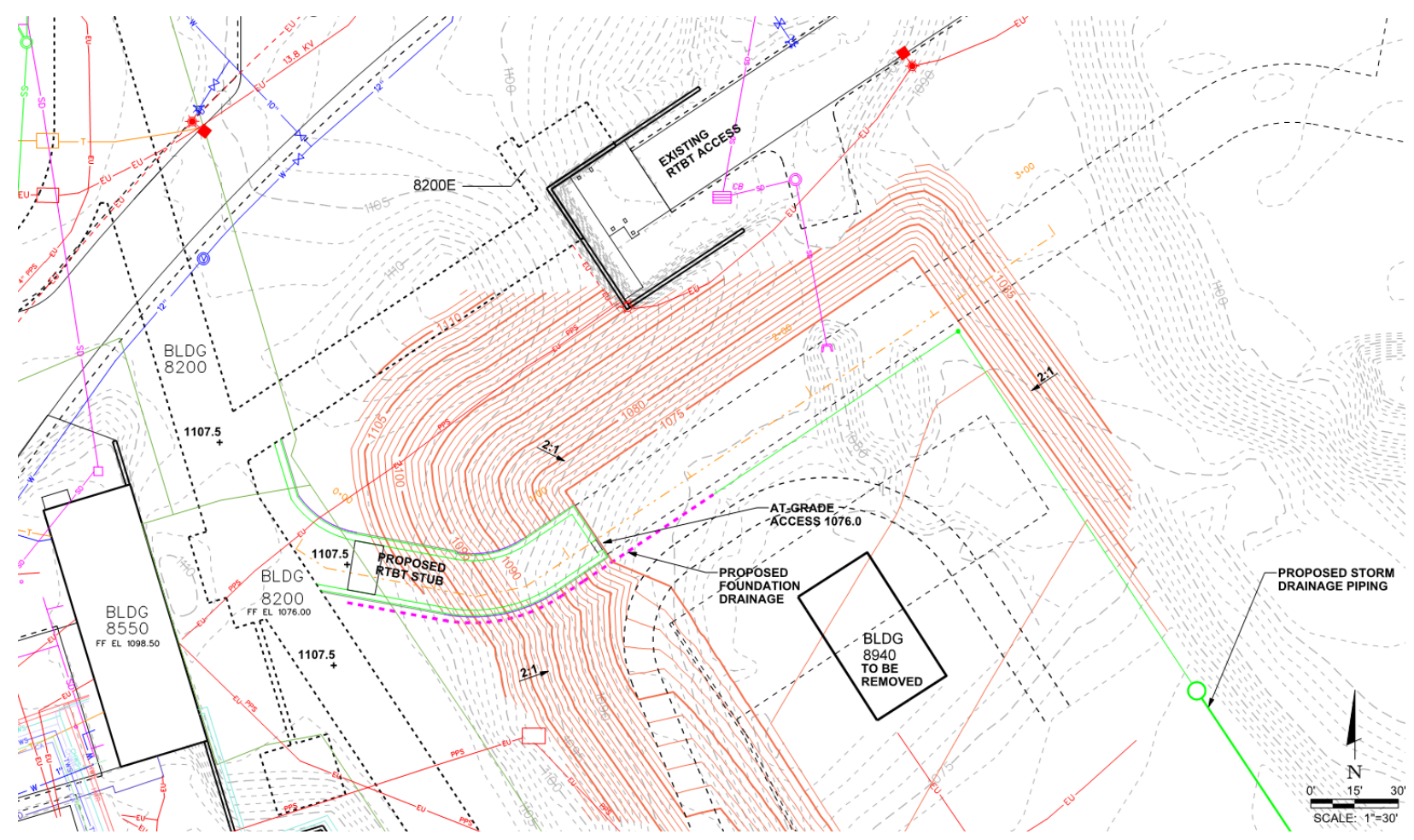

Figure 7.6. Backfill plan and new stormwater piping. 


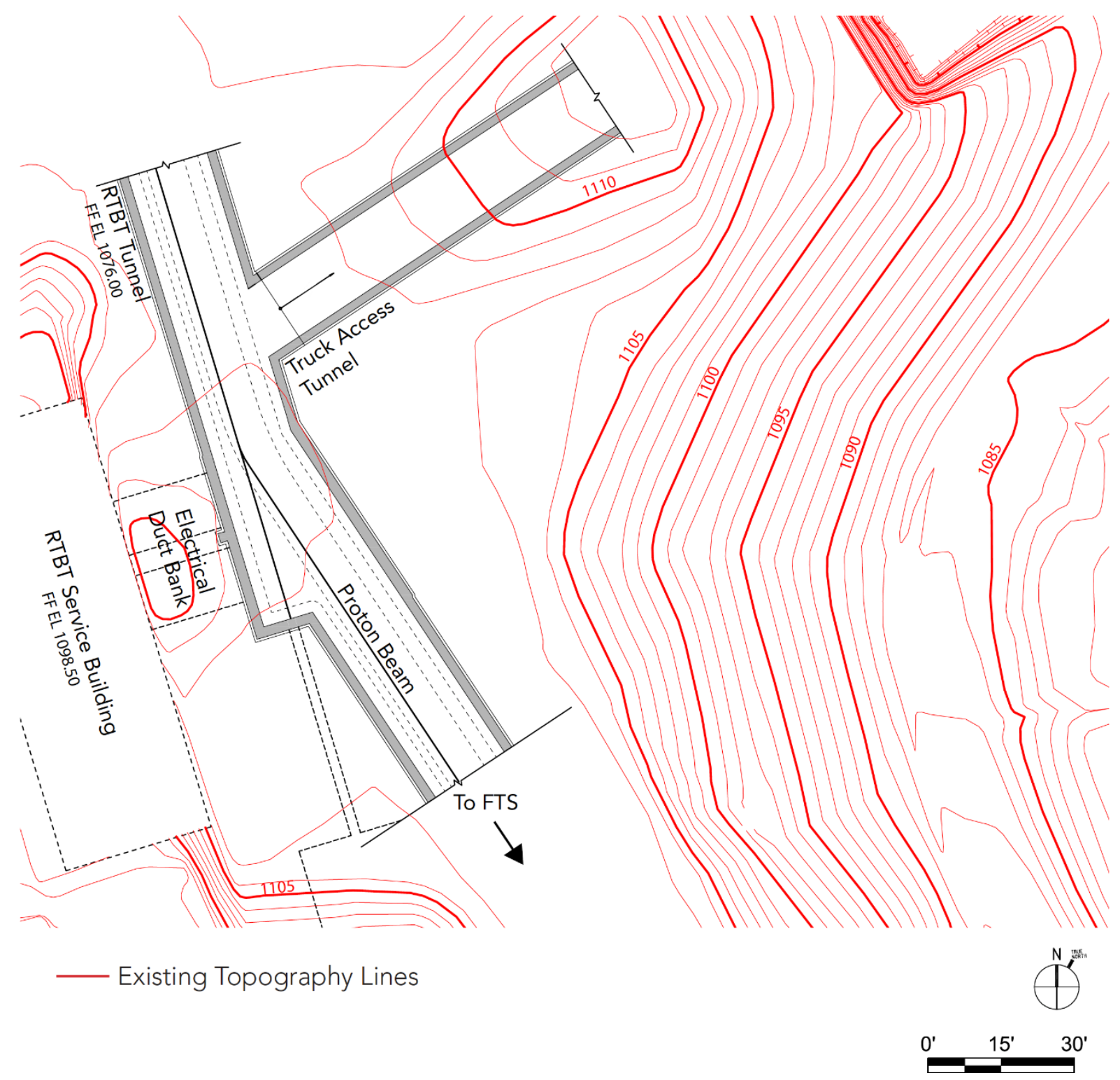

Figure 7.7. The existing RTBT tunnel with existing grades indicated.

The RTBT stub construction will be performed in a step-by-step process as illustrated in the following paragraphs. Each phase number represents a construction step.

\section{Phase 1: Stub excavation}

Based on the planned shutdown of the SNS accelerator for an extended period to allow for the PPU installation primarily in the ring, the site work staging and initial excavation for the RTBT stub could start before the shutdown. Any site work outside of the earthwork shielding dimension could be started and logistically developed up to that standoff location. At the shutdown time, the site excavation could continue to the existing tunnel, including removing the geomembrane and removing the soil down to the approximately 7 feet of cover on the existing tunnel roof. Removing that much soil would alleviate the gravity load to allow for the construction on the east side of the RTBT tunnel, as indicated in Figure 7.8. Stopping the excavation well above the tunnel roof would reduce the rebound effect associated with removing the weight of the overlying soil from the tunnel. 


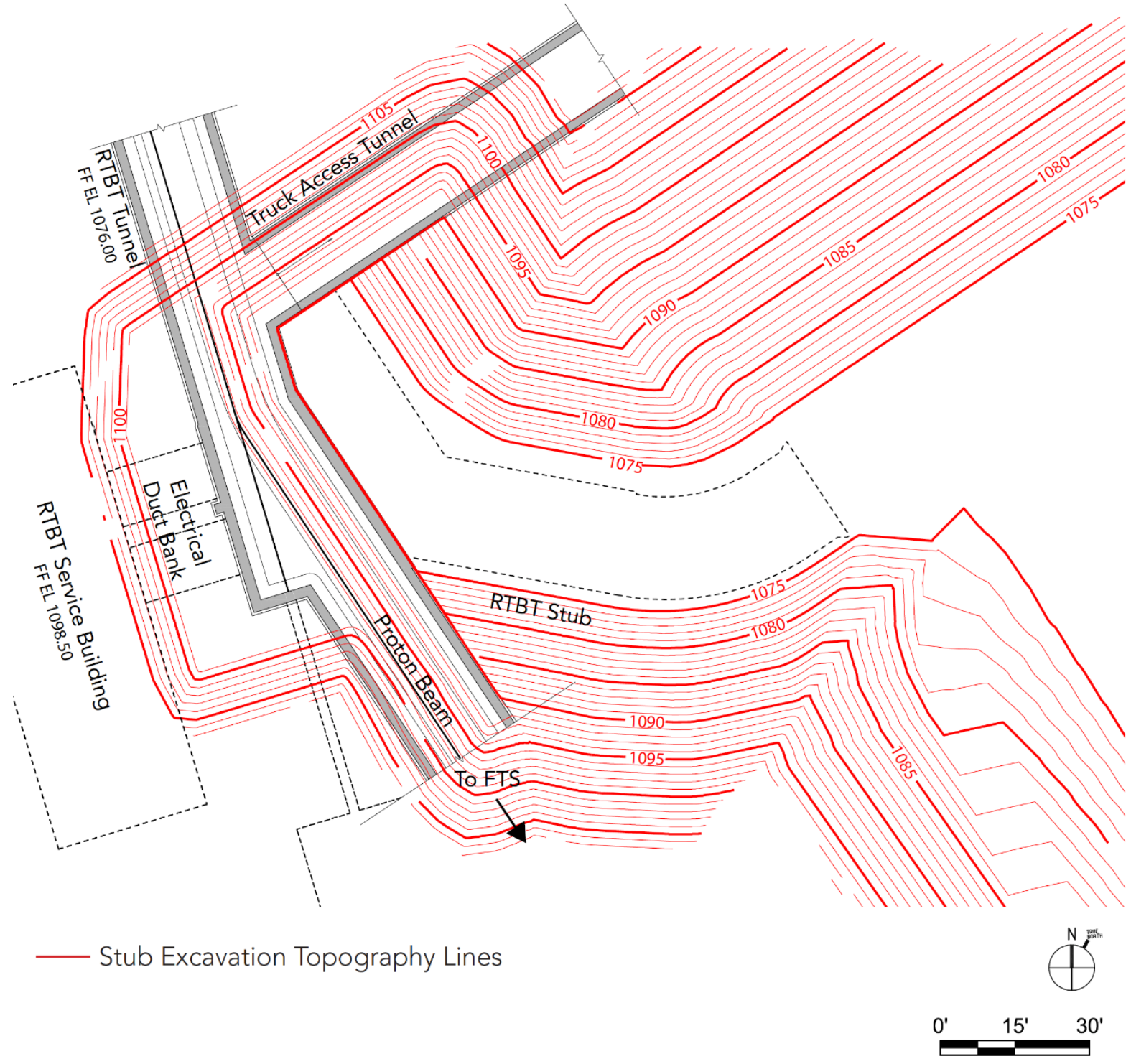

Figure 7.8. Initial stub excavation plan.

\section{Phase 2: Stub construction}

The cast-in-place concrete work forming the floor/foundation, the walls, and then the roof can proceed. The openings into the truck access tunnel can be cut and reinforced as required either before or after the stub is constructed. The Mechanical, Electrical, and Fire Protection system can be installed, and the temporary shielding will be built across the stub to allow the future extension work to proceed up to this point before an additional beam shutdown. The temporary concrete masonry infill wall with secure access door will finish the tunnel stub end. 


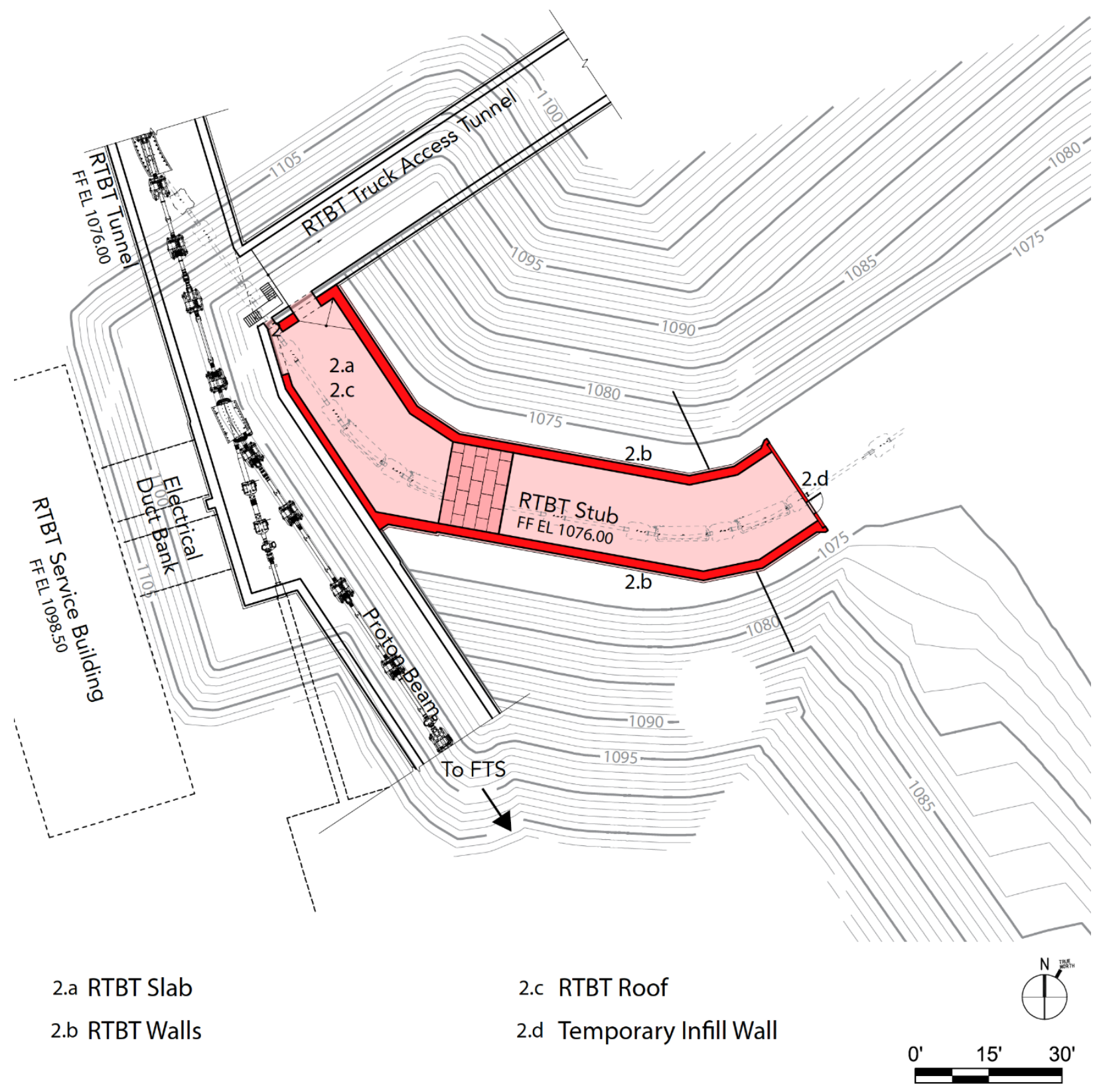

Figure 7.9. Stub construction plan.

\section{Phase 3: Final grading}

Once the concrete tunnel has cured to an adequate level of strength and the tunnel stub waterproofing work is completed, the partial burial and regrading work can proceed. The portion of the stub closest to the RTBT tunnel will have the full earth shielding dimension, placed with the grade sloping down. This grading configuration will allow east tunnel end access and will allow proper water drainage to the south, as shown in Figures 7.10 and 7.11. The displaced portion of the geomembrane over the existing tunnel will be replaced, along with a small portion at the west end of the stub.

See Section 7.2.3 for a discussion of the earth shielding required at the end of the PPU project. 


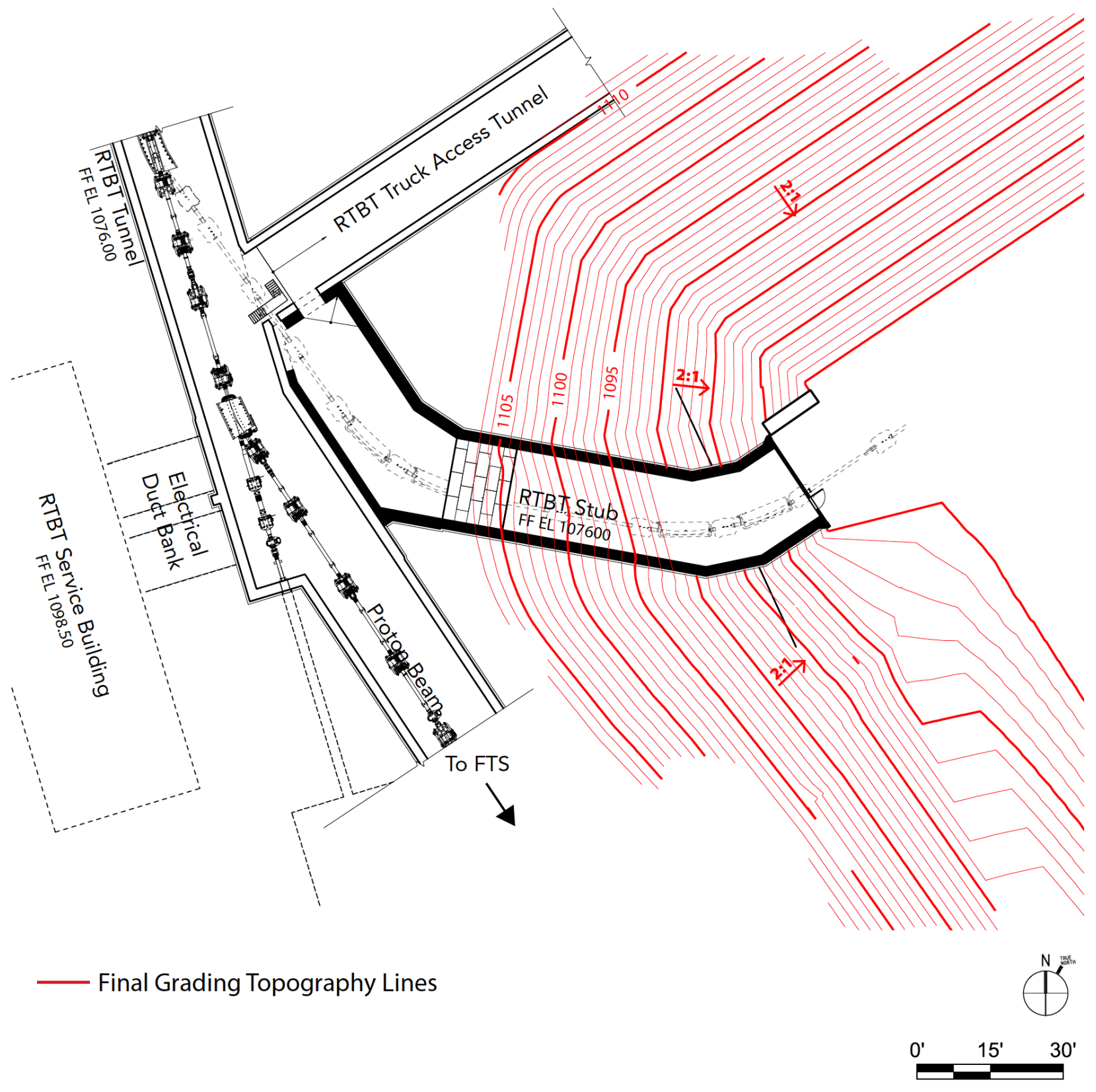

Figure 7.10. Final grading plan. 


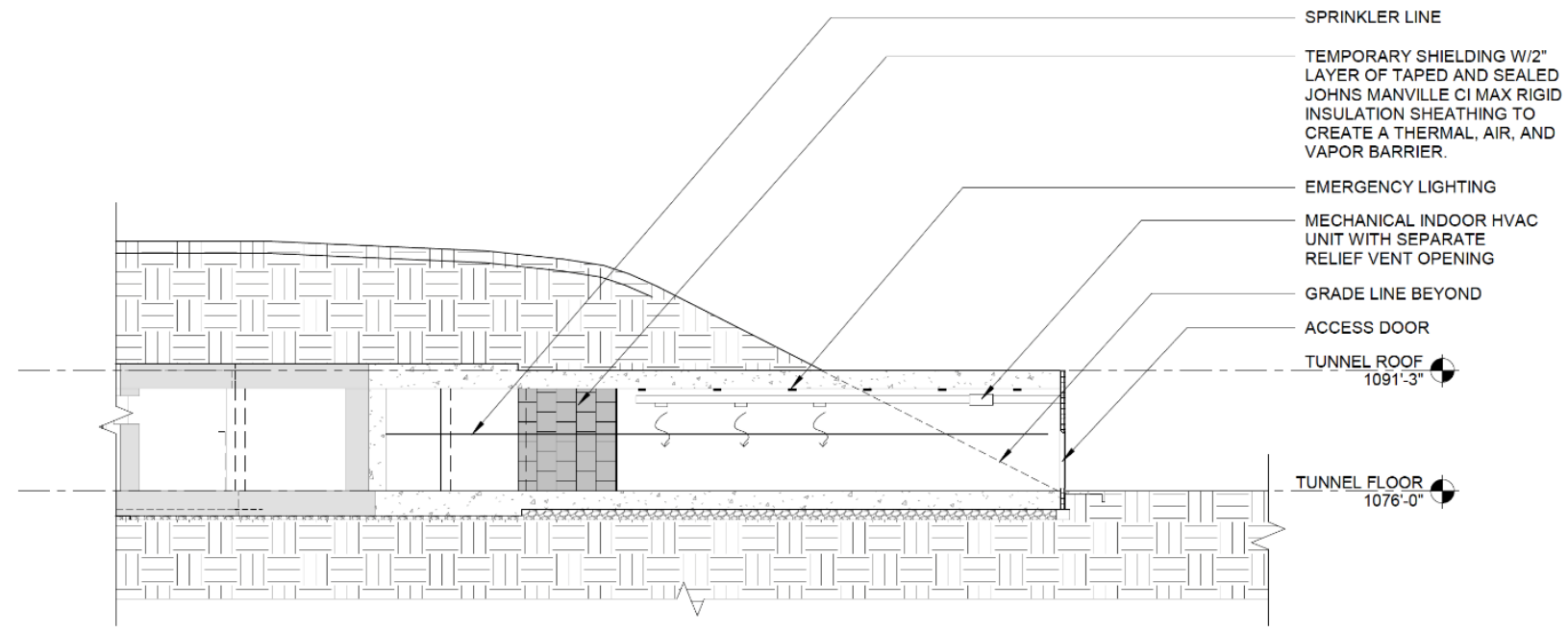

Figure 7.11. Elevation view of tunnel after final grading.

\subsubsection{Architectural/Structural}

The RTBT stub is a 2,600 gross square feet concrete tunnel structure similar to the existing RTBT tunnel. It will be constructed to enable the completion of the R2T2 tunnel as part of the STS project. At completion of the RTBT stub construction, the stub will be primarily below grade with on-grade access. It will not house any accelerator components as part of the PPU. During the STS project, the stub will be connected to the R2T2 tunnel. It will be completely below grade and will house approximately 100 lineal feet of the STS proton beam line.

The proposed section configuration for the structural connection is indicated in Figure 7.12. This will require removal of two sections of the existing RTBT truck tunnel wall to allow the transition of the proton beam into the adjacent RTBT stub and allow for proper access in the tunnel. The wall openings are for the proton beam, utilities and personnel and equipment access. The opening for the proton beam and utilities is proposed to be approximately 2 feet wide and 7.5 feet high with no supplemental reinforcing needed. The access opening will be approximately 6.5 feet wide and 7.5 feet high and will require a supplemental steel structure at the wall opening consisting of a squeeze lintel.

The new RTBT stub building will be reinforced concrete (IBC Type 1B construction). Exposed structural steel will be provided that has a 2 hour fire-rated intumescent coating.

The new stub and the impacted existing tunnel will be waterproofed and a geomembrane installed that is compatible with the one in the existing system. Foundation drains like those in the RTBT tunnel will be provided for the new tunnel. 


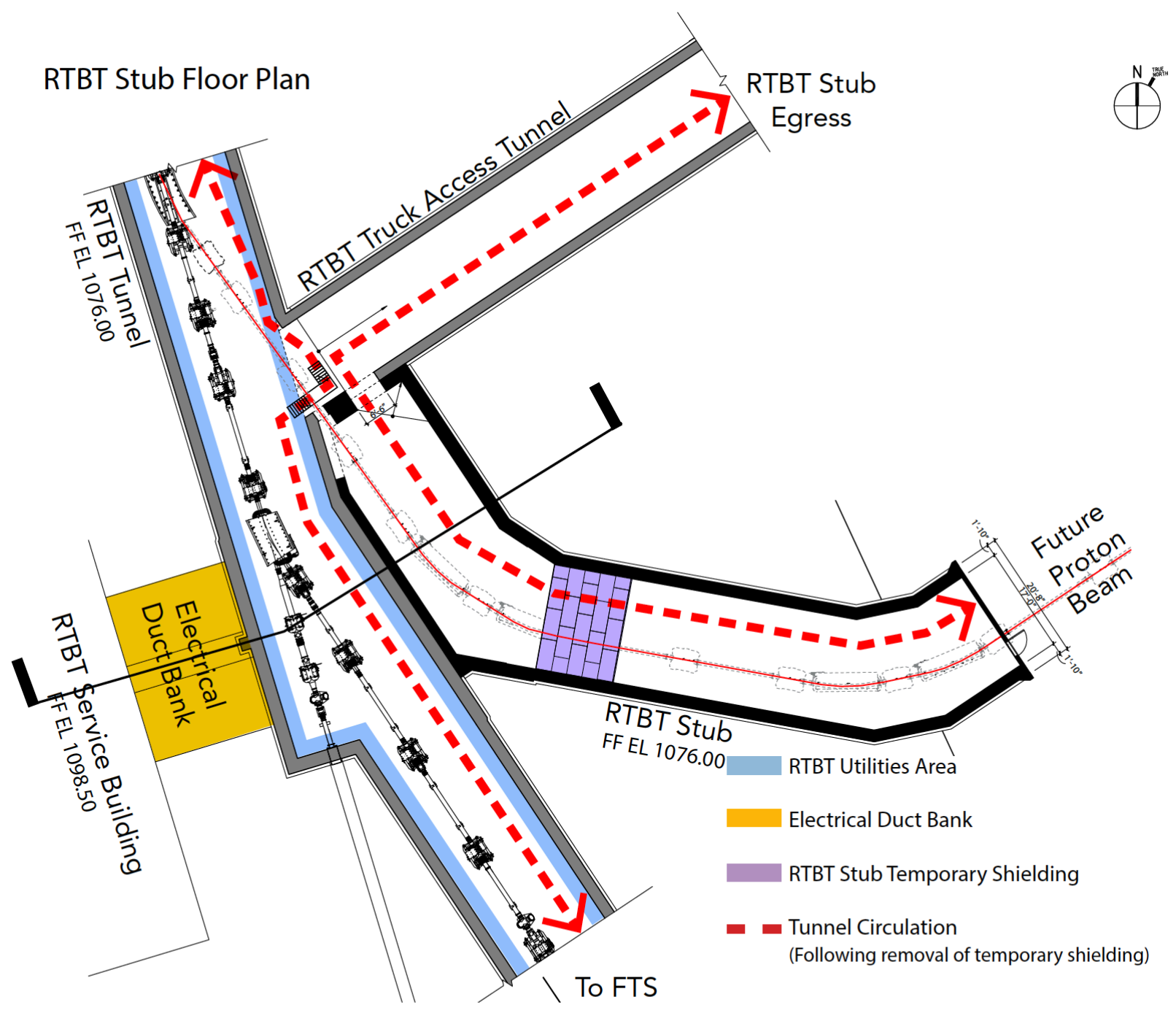

RTBT Service Building, RTBT Tunnel and RTBT Stub Cross Section

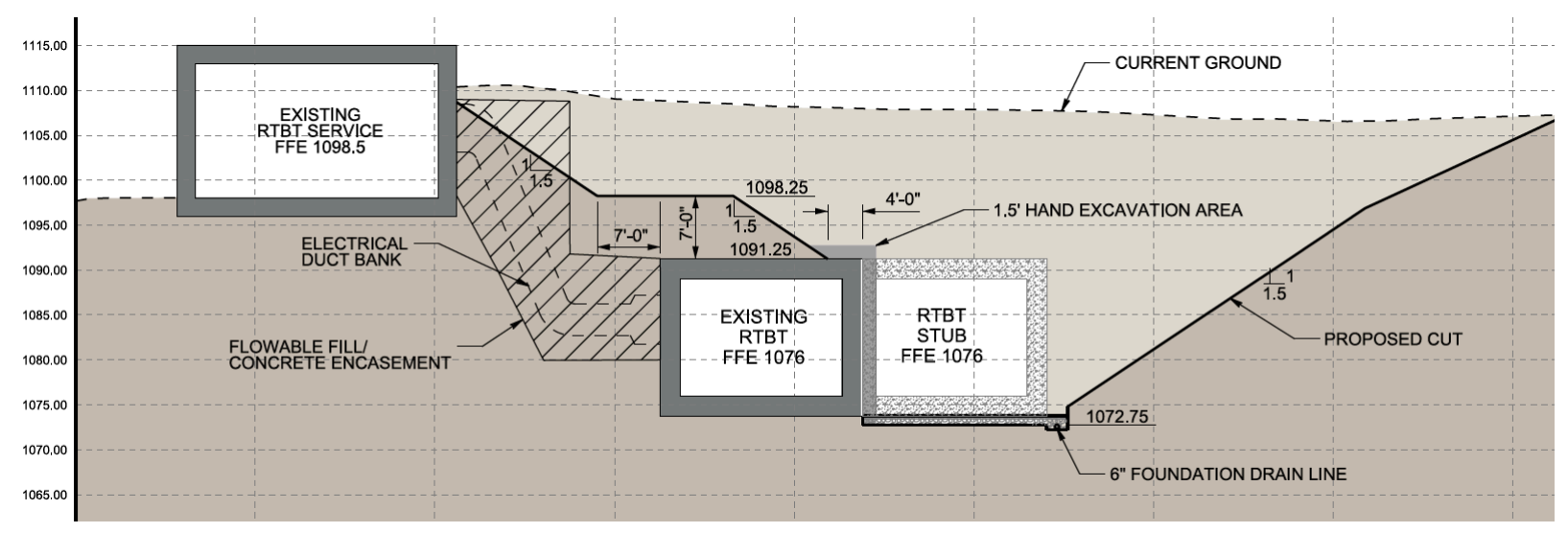

Figure 7.12. RTBT stub structural connection plan. 


\subsubsection{Electrical}

Minimal electrical utilities will be provided to serve the RTBT stub, including ambient lighting, convenience receptacles for portable equipment, and power for the HVAC unit. The source of power for this equipment will be extended from the existing RTBT tunnel, as indicated below.

The $277 \mathrm{~V}$ light fixtures will be industrial, weatherproof LED work lights switched ON/OFF at the entry to the stub. A 20-foot-candle illumination level, on average, will be provided within the tunnel. The switch will have a pilot light to indicate lights ON or OFF. Normal and emergency power for the lights will be provided from existing light fixtures in the RTBT tunnel.

Several $120 \mathrm{~V}$ convenience receptacles will be provided in the tunnel. Power for the convenience receptacles will be provided from an existing circuit inside the RTBT tunnel. The HVAC system will be $480 \mathrm{~V}, 3$ phase. A local disconnect will be provided for the HVAC unit. The power for the HVAC will be routed from panel RS-1P1, located in the RTBT service building. An existing spare conduit will be used to route the power between the RTBT service building and the RTBT tunnel.

\subsubsection{Mechanical}

Heat will be required in the RTBT stub to preclude the fire protection piping from freezing. An HVAC unit will be provided to provide heat, circulate air, and minimize moisture. The unit will be installed on grade outside the end of the stub. A duct will be routed inside the stub to provide outside air in the tunnel. A thermostat will be provided to control temperatures.

\subsubsection{Site Utilities}

The approximate locations of existing utilities within the limits of the proposed stub are shown in Figure 7.6. The only existing site utility within the limits of the stub construction is a PPS circuit extending from the RTBT access tunnel area to the RTBT service building and then to the RTBT tunnel access stair. Outside the construction limits but nearby to the south of the stub area is high-voltage underground power cabling in duct banks that serve the Target Building.

A new stormwater collection header will be installed to accommodate drainage from the reconfigured site.

\subsubsection{Life Safety}

The east section of the new RTBT stub building will be separated from the RTBT tunnel by noncombustible stacked shielding. Exit access east of the stacked shielding will be provided directly to the outside via a man door in the east wall of the RTBT stub in compliance with NFPA 101, Life Safety Code. Exit access west of the stacked shielding will be provided via the existing RTBT truck access tunnel (RTBT-01) and the existing RTBT emergency egress ladderway (RT-103).

\section{Fire Protection Systems}

\section{Sprinklers}

Piping will be extended from the existing RTBT truck access tunnel (RTBT-01) and new sprinklers provided in the new RTBT stub building. The occupancy hazard classification will be Ordinary Hazard Group 2 (0.20 gpm over 1,500 square feet), and the sprinkler system design will comply with NFPA 13, Standard for the Installation of Sprinkler Systems. Seismic bracing for sprinklers will be provided. The installation of seismic bracing will be in accordance with NFPA 13. 


\section{Fire Alarm}

The fire alarm notification circuit in the existing RTBT truck access tunnel (RTBT-01) will be extended into the new RTBT stub building. A manual pull station will be provided at the east exit door.

\section{Fire Extinguisher}

A $10 \mathrm{lb}$ ABC dry chemical fire extinguisher and cabinet will be provided at the east exit of the RTBT stub.

\subsubsection{STS Interfaces}

Plans do not call for any of the tunnel included in this scope to have lateral penetrations for utility supply. The STS project will install the new utility distribution required for the future R2T2 beam line. The STS project will also install the crane in the tunnel; the PPU will install only roof embeds to facilitate the future crane installation.

\subsection{ASSUMPTIONS}

During subsequent PPU design phases, it is anticipated that the following assumptions, which may impact project scope or design approach, will be further evaluated:

\subsubsection{Klystron Gallery Assumptions}

No new storage buildings are needed.

No new HVAC equipment is required for the existing RF equipment running at higher power. It is assumed the existing klystron gallery may need to run at a slightly higher temperature, and any equipment temperature issues will be addressed separately from the PPU by localized cooling and ongoing operational improvements.

There is no CF scope for the remainder of the klystron gallery.

No new HVAC is needed in the Ring Service Building as a result of increased heat loads from the new equipment required for the accelerator upgrades that will operate at a higher proton beam energy.

The area of the klystron gallery (KL-143) will be cleared out and the new chases installed before the CF construction. There will be no other work in the klystron gallery (KL-143) for the duration of the CF construction.

\subsubsection{RTBT Stub Assumptions}

Geotechnical evaluations of soil at stub area will have similar properties to previous borings in the area, and therefore no load transfer platform or other deep foundations will be required.

The RTBT stub will be constructed in the dry part of the year (May-November) to minimize rain delays that might extend the shutdown period. 



\section{RESEARCH AND DEVELOPMENT}

\subsection{RING FOIL INJECTION}

Section 5.1 highlighted the charge exchange foil concerns associated with doubling the ring beam intensity. In addition to the issue of foil survivability from a heating standpoint, issues such as foil holder robustness against reflected convoy electrons and against mechanically driven fluctuations (thermal transient-driven and/or charge buildup-driven) are concerns. The PPU project will investigate the foil survivability and mechanically driven fluctuation issues using the foil test stand, which is already established, shown in Figure 8.1. It is a desktop apparatus that uses a $30 \mathrm{keV}$ electron beam capable of an energy deposition density in the foils that exceeds that of the $1.3 \mathrm{GeV}, 2.8 \mathrm{MW}$ PPU beam. Stripper foils with different corrugation patterns (to prevent curling), thicknesses, and crystalline structures will be tested to determine operational robustness at the PPU beam intensity.

The PPU project will also measure the in situ temperature of the stripper foils currently in use. This will be done using a two-wavelength detector system mounted to a telescope located in the Ring Service Building. The telescope views the stripper foil through a chase located between the Ring Service Building and the ring tunnel. Measuring the signal intensity at two wavelengths enables the temperature measurement to become independent of the stripper foil emissivity. This is an important part of the measurement, as the foil emissivity is unknown and changes as the foil ages. Once the foil temperature is known, we will extrapolate the result to PPU beam intensities and then use that result to predict the stripper foil lifetime. This work will also check that the foil test stand is replicating the actual in situ conditions.

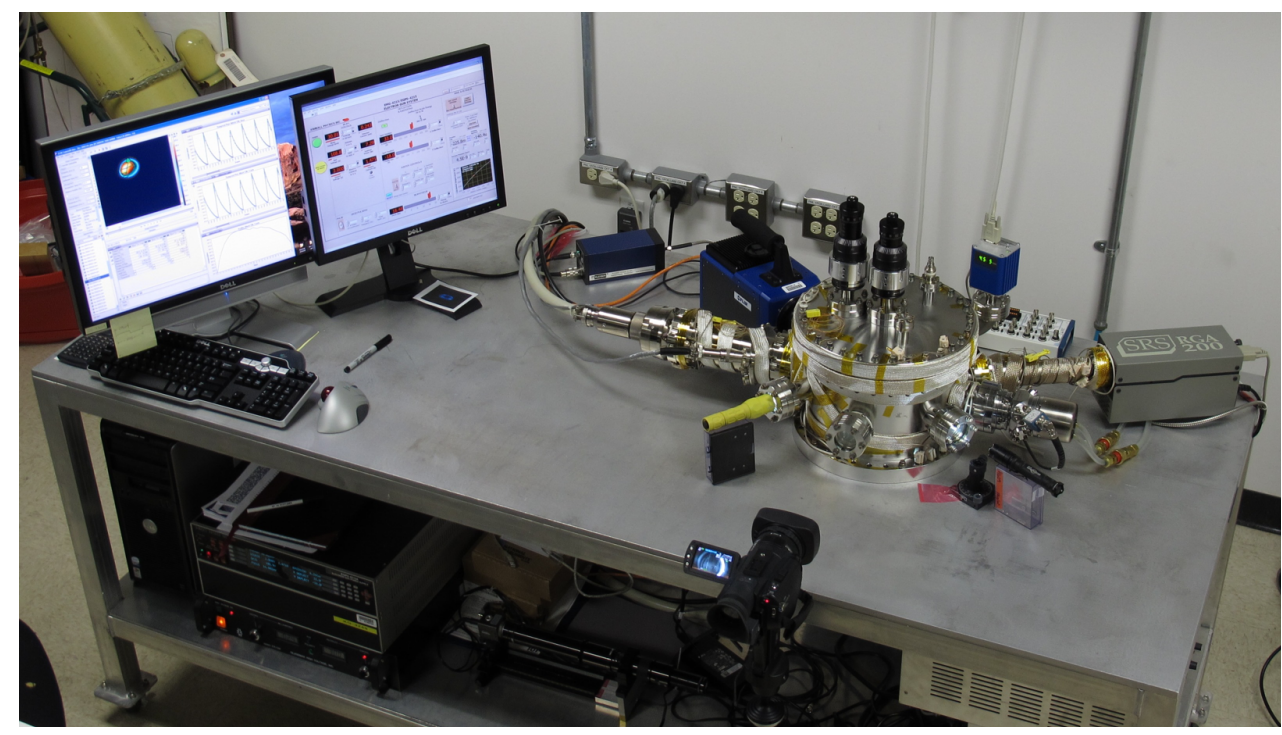

Figure 8.1. The foil test stand laboratory.

\subsection{MERCURY TARGET HIGH-RATE GAS INJECTION DEVELOPMENT}

This work scope covers the development of high-flow gas injection for maximum mitigation of cavitation erosion and reduction of beam pulse-induced fatigue stress in the target vessel. It does not include scope associated with orifice bubblers, injected gas rates $\leq 1$ SLPM, or once-through gas supply associated with 
the ongoing gas injection initial implementation (GI3) effort. Included are development of high-gas-rate bubblers and protective gas walls; also included is gas removal development to ensure safe and efficient mercury process loop operation. Operation of any test facilities and the documentation of results are covered in this effort. The plan is based upon the envisioned deployment of target gas injection for the long term, as described in the Conceptual Design Report for Mercury Target Gas Injection [1].

R\&D will be conducted during the PPU project to optimize target gas injection for pressure wave and cavitation damage mitigation in the SNS target and to ensure safe operation of the mercury loop. The efforts that will be pursued to achieve these two goals are described in the sections below. Most of the experimental work will be performed at the TTF and at the Thermal Hydralic Labatory (THL). The TTF is a full-scale mercury test loop that is mostly prototypic of the SNS mercury process loop. It includes the mercury-target flow loop piping, a similar mercury pump, and a reconfigurable test target region. Because of the difficulty of measuring bubble populations in mercury, a full-scale water test loop was fabricated to measure the flow fields and the bubble distribution inside the target at the THL. The THL is located on the main campus of ORNL and is equipped with a wide range of instruments relevant to our work, such as high-speed video and stereo particle image velocimetry. The targets at the TTF and the THL are shown in Figure 8.2.
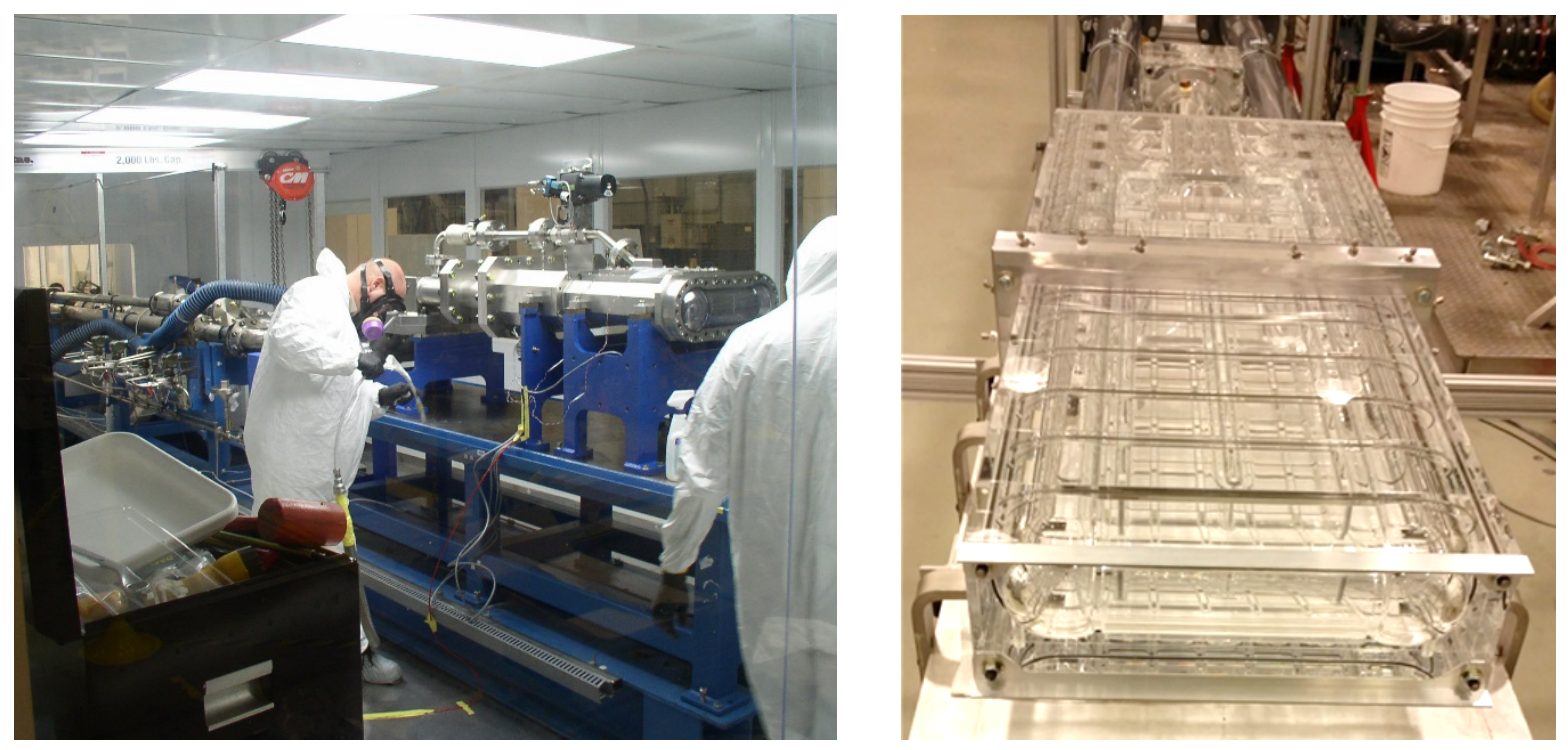

Figure 8.2. Photos of the mercury target at the TTF (left) and the water target at the THL (right).

\subsubsection{Small Gas Bubble Injection and Protective Gas Layer}

Gas injection through small orifices (micrometer) size has been extensively investigated by SNS target development staff. It has known limitations: very small orifices are needed to generate small bubbles, many orifices are needed to achieve a high volume fraction, it requires high backpressure, and the systems are relatively complex to build [2], [3], [4]. Because of all these disadvantages, SNS has also investigated the gas injection method implemented at J-PARC (see Figure 8.3, left): the swirl bubbler. Rather than relying on orifices to generate the bubbles, a swirling flow is used to shear a gas vortex line into small bubbles. The swirling flow is generated using a fixed vane (see Figure 8.3, right), and gas is injected in the low-pressure region just downstream of these vanes. 

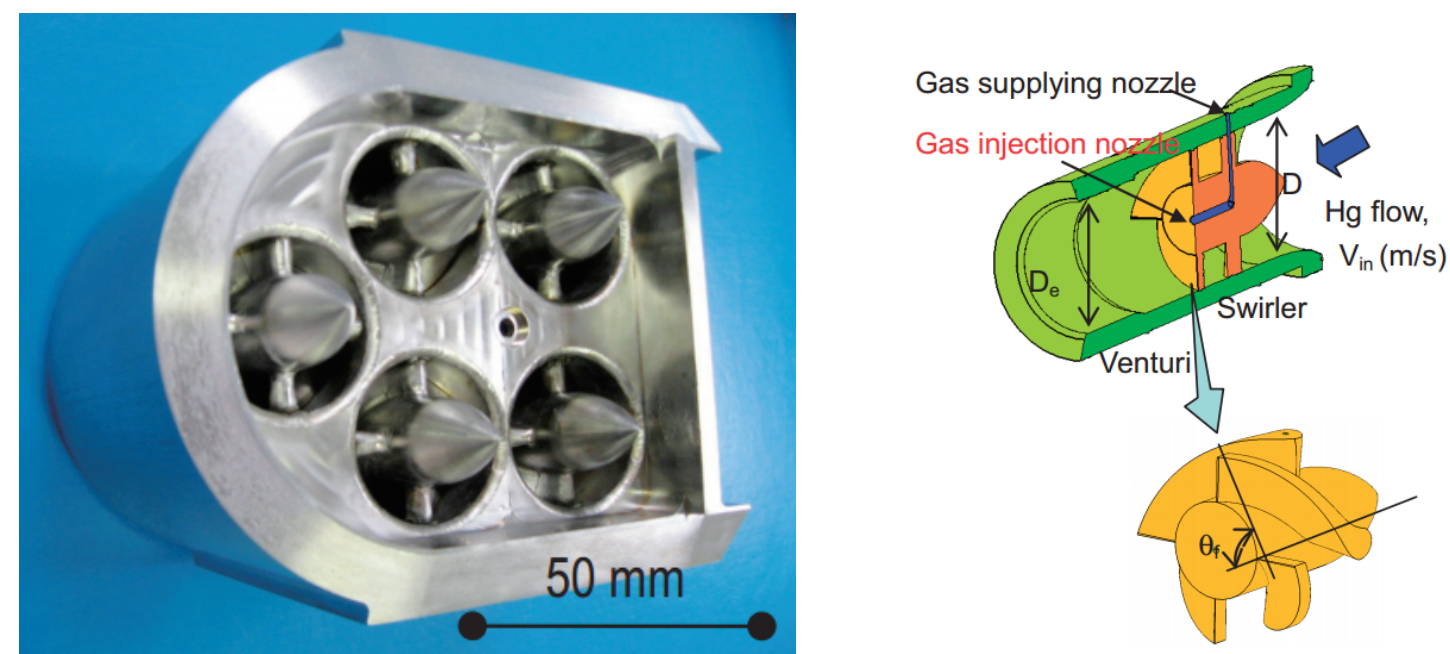

Figure 8.3. Picture of the swirl bubblers assembly used at J-PARC (left) and schematic of the crosssection of a single swirl bubbler (right).

Preliminary designs that will fit inside SNS target bulk inlets have already been developed and tested in water (Figure 8.4). Three-dimensional stainless steel swirl bubblers have been successfully printed and will be tested at the TTF soon. Additional research is needed to optimize the swirl bubbler design to generate the desired gas bubble population without impacting proper cooling of the target vessel. Experiments will be performed in water and mercury to measure the bubble size population and pressure loss for several swirl bubbler geometries. Lessons learned from GI3 operation will be incorporated in the development of high-gas-rate bubblers. In addition, numerical work will be performed to ensure that the vessel is properly cooled if swirl bubblers are used and that the bubbles are properly distributed in the target.
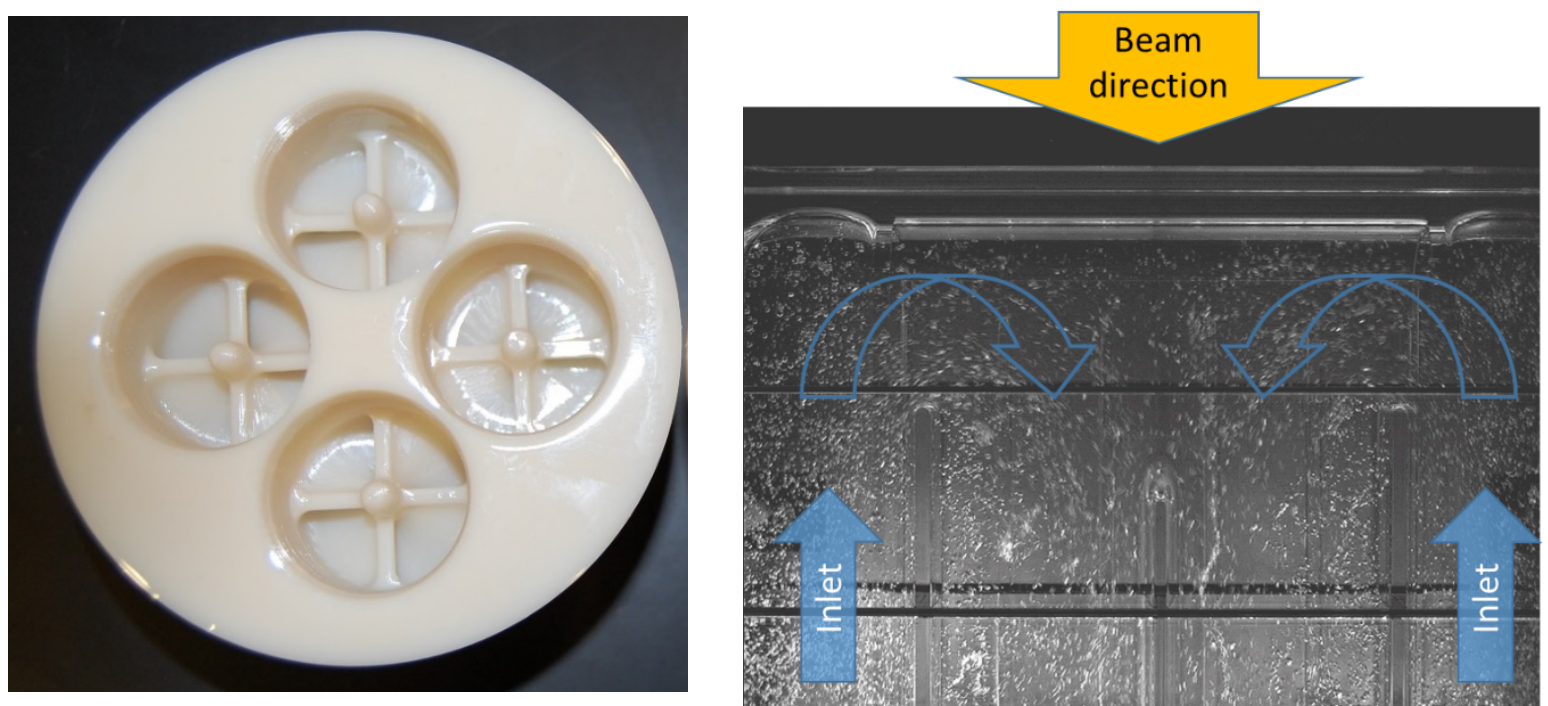

Figure 8.4. Swirl bubbler prototype developed for SNS target that was tested in a water loop (3D printed model on the left. On right is an example top view of bubbles flowing in water test target.

With the PPU beam on the mercury target, a protective gas layer on the inner window wall of the target needs to be implemented to ensure maximum mitigation of vessel erosion in addition to the mitigation 
that will be provided by small gas bubble injection. In experiments done on gas walls with pulsed beams, gas walls were highly effective in reducing cavitation damage. But they are more difficult to engineer into a production target. Three approaches have been investigated at SNS for establishing a gas layer:

- Free gas layer: gas is injected locally on the inside of the target wall with no structural modification (Figure 8.5).

- Surface-modified gas layer: surface is textured to enhance gas layer attachment at the wall (Figure 8.6).

- Porous wall gas layer: a porous material is used to distribute gas across the vulnerable wall (Figure 8.7).

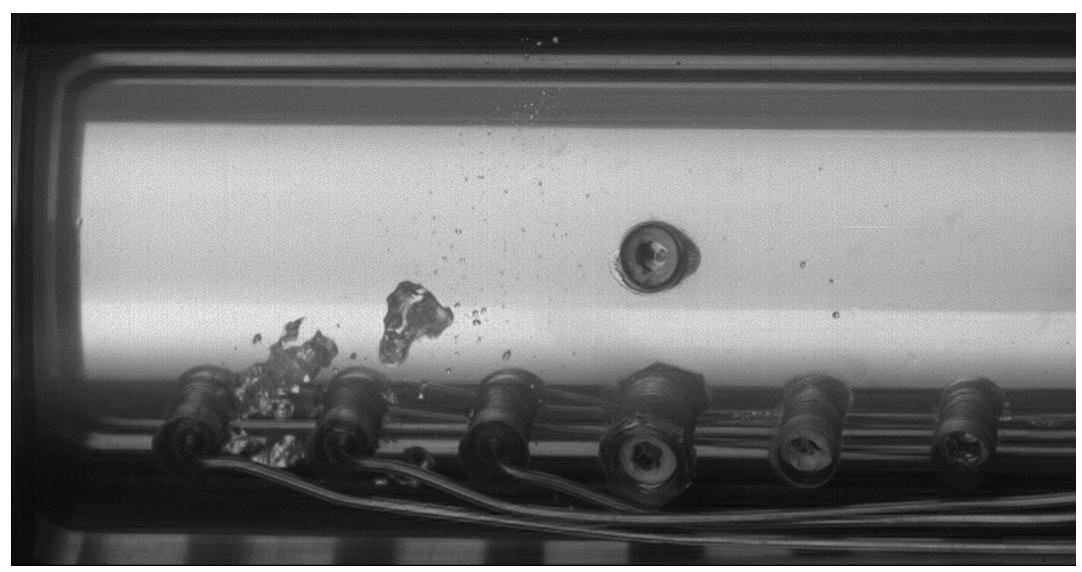

Figure 8.5. Free gas layer experiments at TTF on an original target design.

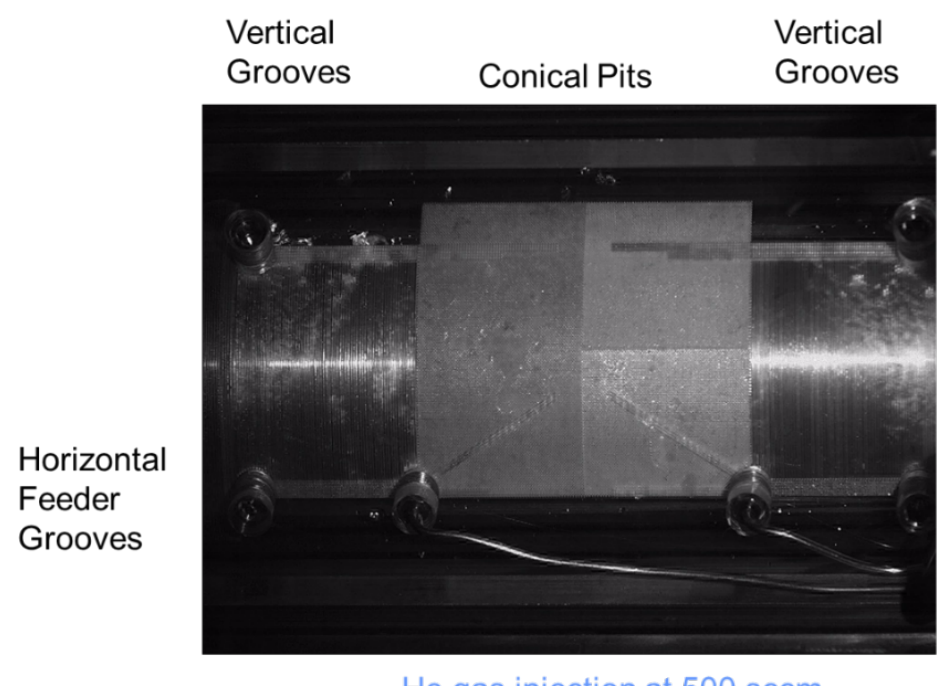

He gas injection at $500 \mathrm{sccm}$

Figure 8.6. Example of textured surface tested at TTF. 


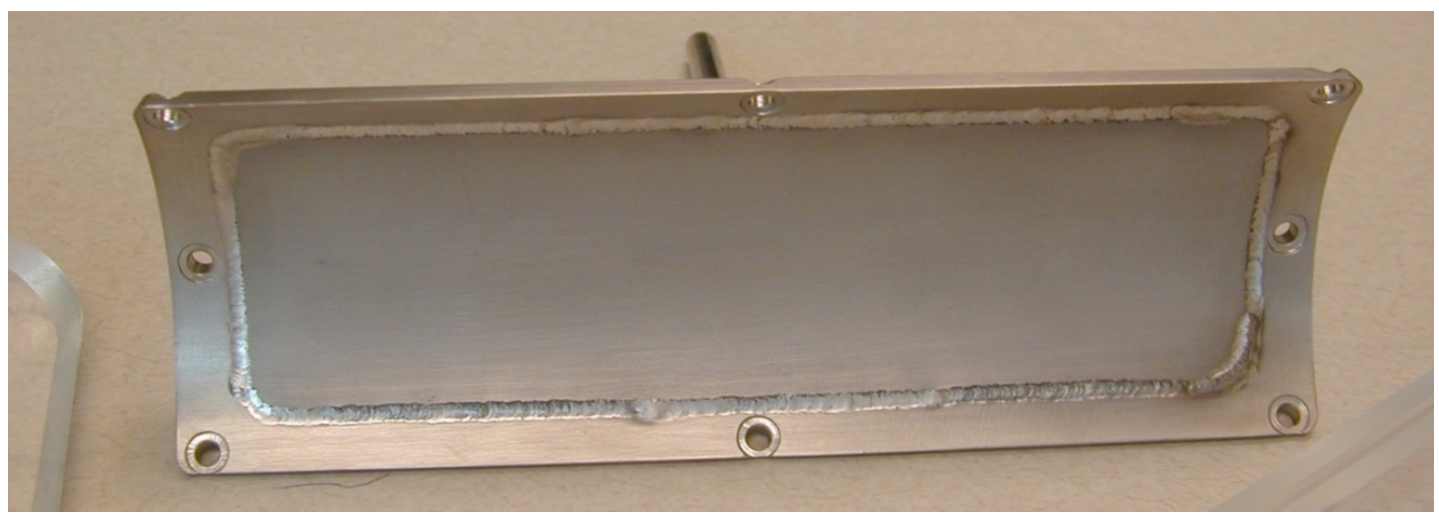

Figure 8.7. Example of porous media test at TTF.

Establishing a free gas layer to cover the whole nose section was found to be challenging in the original target design. However, in the new jet-flow target design (see Figure 8.8), the jet flow will help stabilize the gas layer and enable better results. The textured surface approach gave very promising results, but uncertainty remains with regard to the mercury's surface wetting behavior on the steel vessel wall in a real SNS target environment versus the experiment. The wetting behavior could switch from non-wetting to wetting, which would degrade the gas wall coverage. Furthermore, the textured features present some risk as fatigue stress risers.

Uncertainties remain regarding the porous media, too, as there is no indication that the material will last in the beam and allow proper cooling of the window. Consequently, the free gas layer method will be the main approach pursued. Fortunately, in-beam experiments with a gas wall indicate that less-than-perfect gas wall coverage can still be highly effective. The TTF target will be upgraded to a jet-flow design, and several gas wall nose injection methods will be tested. The gas wall layer and small gas bubble interaction will be also investigated to ensure that the gas wall layer does not affect the bubble size population generated by the swirl bubbler.
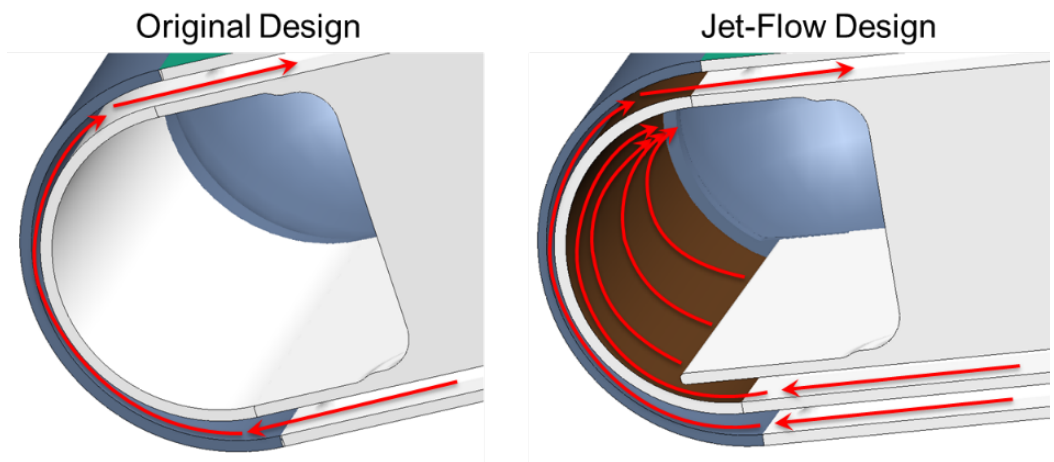

Figure 8.8. Schematic of the original and jet-flow target designs and their associated flow patterns. 


\subsubsection{Gas Removal in the SNS mercury loop}

One of the main concerns with gas injection in the SNS loop is the potential for gas accumulation in the process piping and heat exchanger that could displace enough liquid mercury to overflow the pump tank. The amount of gas that needs to be removed for safe operation with high-rate gas injection will be determined based on development and lessons learned from GI3 experience. The goal is to modify the mercury process system as little as possible and not to over-engineer the gas removal process.

Tests performed at TTF [5] and associated CFD simulations indicated that steady state displaced mercury conditions could be reached for a low gas injection flow rate ( $<1$ SLPM) relatively quickly (in less than $10 \mathrm{~h}$ ) without any large gas releases. Further experiments with more prototypic bubblers are needed to evaluate whether the same behavior is observed for large gas injection. Confident translation of TTF results to the SNS is not possible owing to some key differences in the process loop designs (Figure 8.9):

- TTF does not have a heat exchanger, only a mockup to match the pressure drop.

- $\quad$ TTF does not have a $3 \mathrm{ft}$ drop on the return line.

- TTF pump inlet is above the impeller, whereas at SNS it is below the impeller and protected by a cover plate.

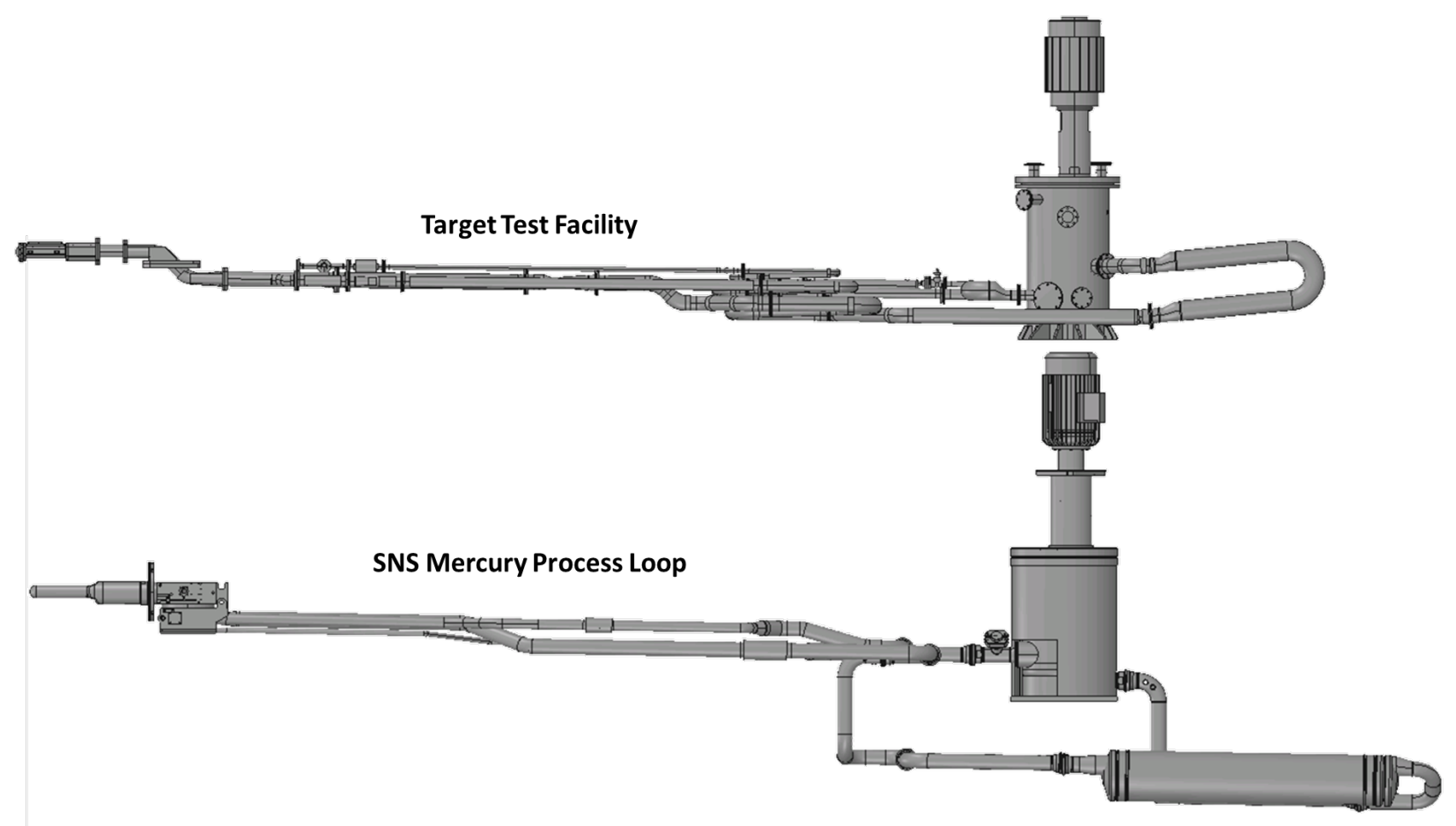

Figure 8.9. Elevation views of the TTF mercury test loop (top) and SNS mercury process loop (bottom).

In addition, although the CFD results provide great insight into where the gas may accumulate, it is not sufficiently accurate to estimate the size of the gas holdup. The limitation results from the presence of both small bubbles and free surface, which makes the modeling very challenging. 
One of the first efforts will be to update the TTF with a more prototypical jet-flow target and - to the extent feasible within the test facility - the process piping configuration. Recently, the target vent line was moved and improved to extract as much gas as possible (see Figure 8.10). Experiments will be performed to evaluate how much the modifications will improve the gas extraction. Another possible upgrade would be the installation of vanes within the target return section to coalesce the bubbles and extract them more easily. The addition of a compact gas-liquid separator will be investigated at TTF to see if the necessary gas removal can be performed mainly on the target. If gas accumulation remains a potential issue, an additional vent line or possibly a cyclonic gas-liquid separator may need to be installed at the $3 \mathrm{ft}$ drop as a last resort.

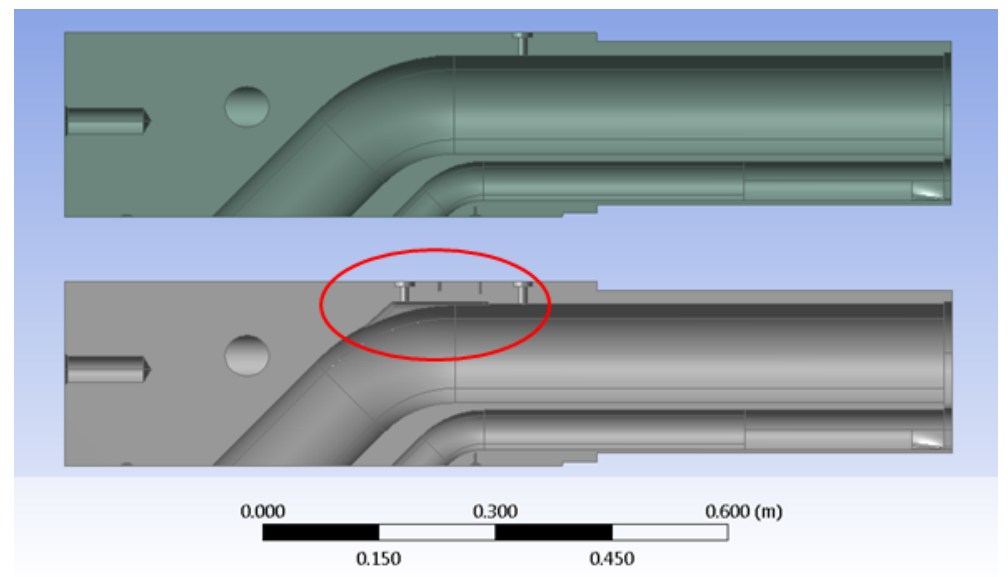

Figure 8.10. Old (top) and new (bottom) location of the target vent line.

\subsubsection{Gas Recirculation}

A recirculating gas compressor is planned for long-term target gas injection to conserve helium and reduce the stack emission potential. Two types of compressor pumps are under consideration: bellows type (similar to that used at J-PARC) and diaphragm type (see Figure 8.11). Two installation locations are currently being considered for the compressor: inside the service bay or in the MOTS equipment room. In the service bay, the compressor would be installed on the wall and would be installed and replaced remotely. It would draw gas from the mercury pump head to a junction downstream of the vapor condenser. If, on the other hand, the compressor were installed in the MOTS equipment room, it would draw cleaned gas from the MOTS discharge, reducing the risk of mercury vapor condensate or highly radioactive gas damaging the compressor low. Compressor maintenance and replacement would be much easier. However, all the target gas flow will be processed by the MOTS, which is designed for handling the envisioned gas injection rate. But there remains a potential for operational issues if a large gas release should occur.

The development of a reliable and maintainable gas compressor system that meets the requirements for target high-flow small bubble generators and protective gas walls will be achieved as part of this R\&D work scope. This work may well include a once-through gas supply as a backup or supply supplement.

Preliminary testing indicates that the Senior Aerospace bellows compressor may not be reliable enough for our application, and the KNF process pump is currently under investigation as a potential replacement. J-PARC has also reported difficulties with Senior Aerospace bellows compressors. Further testing will be performed at TTF to further test the reliability of the compressor. The location of the compressor will then be chosen based on the estimated maintenance required by the compressor. 

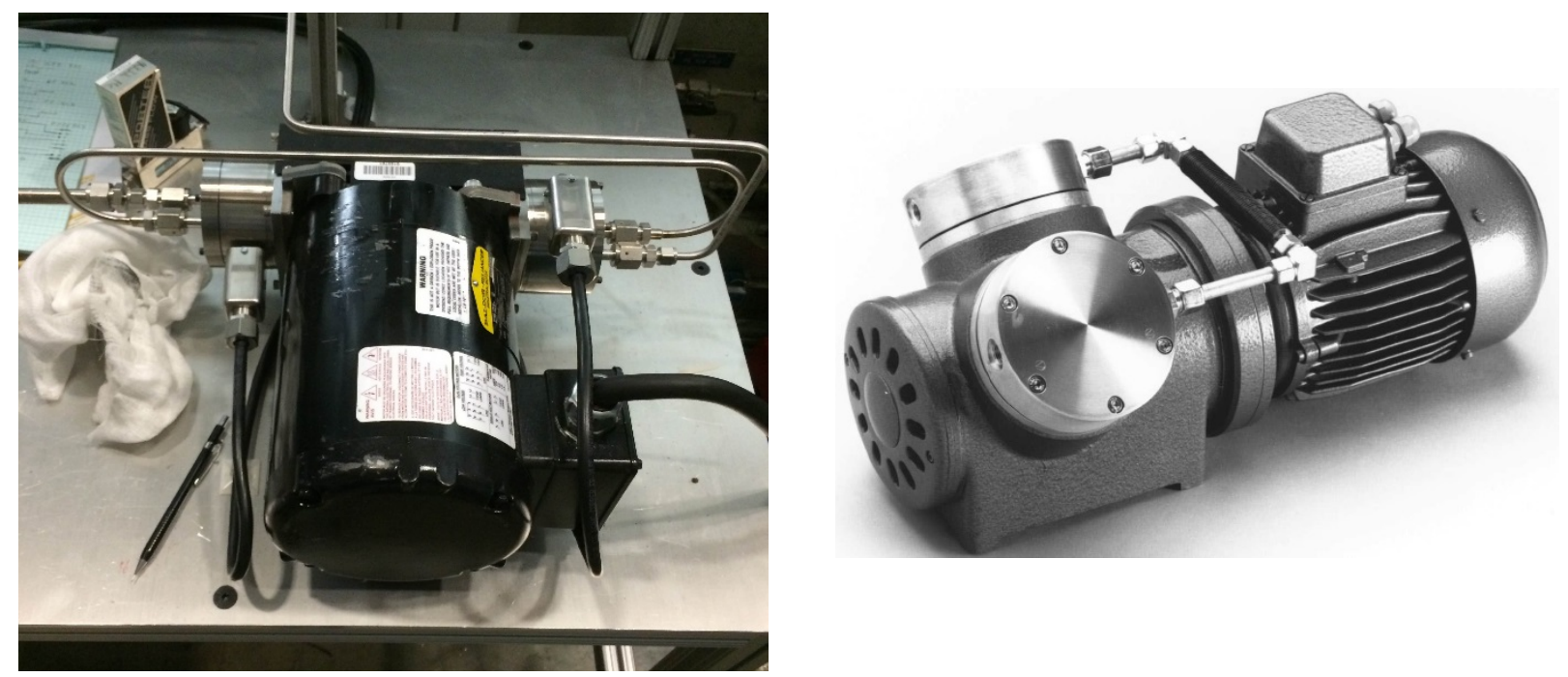

Figure 8.11. A double bellows compressor from Senior Aerospace (left) and a diaphragm compressor from KNF Process Pumps (right). Both have pressure and flow capacities suitable for target gas injection.

\subsubsection{Sensors}

The efficiency of gas injection mitigation needs to be measured. Pressure wave mitigation will be assessed by strain and vibration measurements, and sensors will be developed as another part of an effort of the TRIP. Additional sensors will be developed to monitor cavitation activities.

Because of the potential effects of gas injection on flow rate measurements using venturi tubes, a flow sensor is currently being developed with Delta M Corporation to measure accurately the flow velocity and the temperature in the return pipe. Additional efforts will be pursued to develop this sensor.

\subsection{REFERENCES}

[1] B. Riemer, Conceptual Design Report: Mercury Target Gas Injection, SNS 106010000-TR0132, R00, Oak Ridge National Laboratory, July 2016

[2] M. Wendel, A. Abdou, and B. Riemer, "Choked-flow inlet orifice bubbler for creating small bubbles in mercury," ASME 2013 Fluids Engineering Division Summer Meeting, Vol. 2, Incline Village, Nevada, July 7-11, 2013, American Society of Mechanical Engineers, 2013.

[3] B. W. Riemer, M. W. Wendel, D. K. Felde, et al., "Small gas bubble experiment for mitigation of cavitation damage and pressure waves in short-pulse mercury spallation targets," Journal of Nuclear Materials 450(1), 192-203 (2014).

[4] M. Wendel, B. Riemer, and A. Abdou, "Measurements of gas bubble size distributions in flowing liquid mercury," ASME 2012 Fluids Engineering Division Summer Meeting, collocated with the ASME 2012 Heat Transfer Summer Conference and the ASME 2012 10th International Conference on Nanochannels, Microchannels, and Minichannels, Vol. 2, Rio Grande, Puerto Rico, July 8-12, 2012, American Society of Mechanical Engineers, 2012.

[5] C. Barbier, D. Felde, R. Sangrey, et al., TTF Gas Holdup Experiments, SNS 106010101-TR0019 R00, Oak Ridge National Laboratory, January 2016 


\section{MAJOR ASSUMPTIONS}

This section describes major assumptions for the PPU design. Detailed assumptions for the various subsystems are described in the sections describing these systems. Primary technical assumptions described here relate to the front-end system (ion source) and the choice of which beam parameters to increase to accomplish the power upgrade.

\subsection{FRONT END SYSTEMS}

The PPU power increase will require a peak current of $46 \mathrm{~mA}$ out of the RFQ. This implies a peak current of about $54 \mathrm{~mA}$ delivered by the source, which corresponds to an $87 \%$ RFQ beam transmission rate. No increase above the present beam duty factor is planned for STS operation; it will remain at $60 \mathrm{~Hz} \times 1 \mathrm{~ms}$, or $6 \%$. The baseline plan to reach a $54 \mathrm{~mA}$ source output is incremental improvement of the present ion source design using the existing ion source test stand. The plan for reaching the design RFQ transmission of $87 \%$ at the required ion source output is to use the existing, recently procured new RFQ. Also, a modified chopping pattern waveform procedure will be implemented to produce the required $82 \%$ unchopped beam fraction, as discussed in Chapter 2. These are discussed in the following sections.

\subsubsection{Ion Source}

Ion source beam production currents over recent years are shown in Figure 9.1 (personal communication from Martin Stockli, ORNL, to J. Galambos, ORNL, May 2016). These data are measured at the entrance to the RFQ on the front end of the SNS machine for neutron production sources. Three primary sources are used in operations, averaging about $\sim 50 \mathrm{~mA}$ in recent years with some demonstrating up to $\sim 60 \mathrm{~mA}$ (note that for much of the period shown, there was not a demand for higher beam current, as the beam power was target limited). There has been a steady increase in source performance of $\sim 20 \%$ over the past 3 years. The PPU requirement of $54 \mathrm{~mA}$ has been achieved.

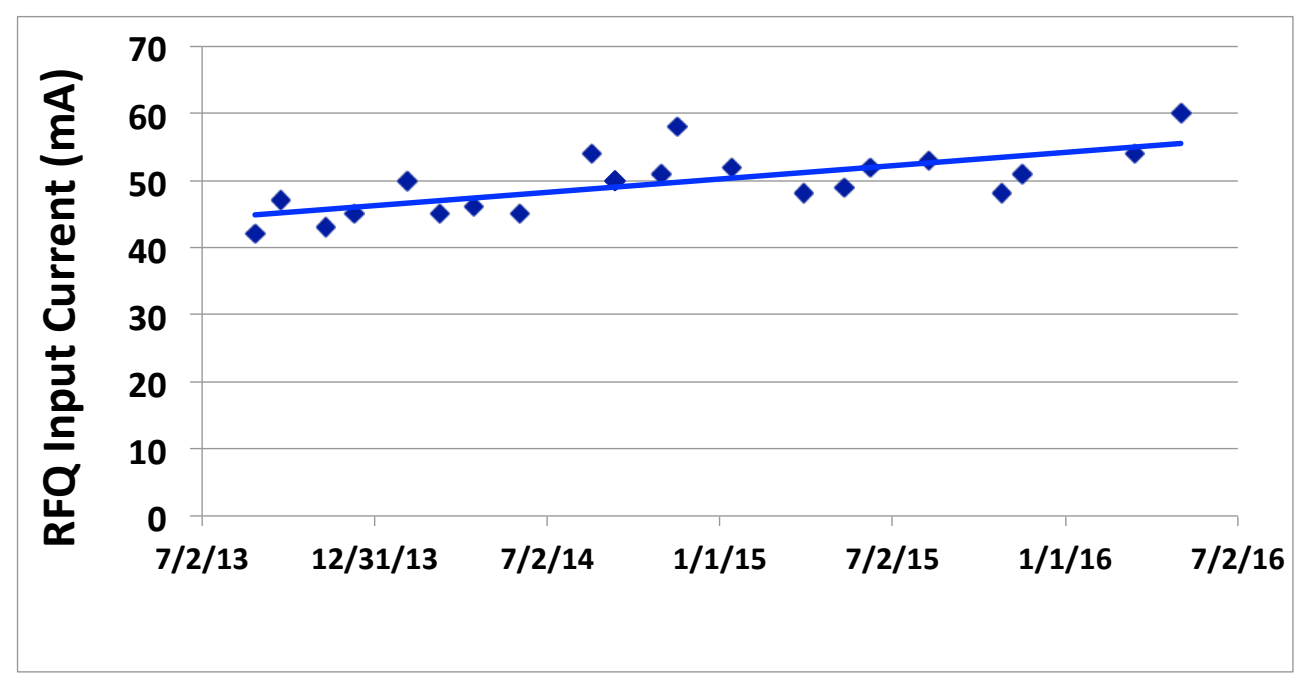

Figure 9.1. Recent ion source current, measured at the RFQ entrance on the SNS front-end.

Increasing the reliable source output to $54 \mathrm{~mA}(\sim 10 \%$ additional performance increase) will be accomplished by 
- increasing the RF power capability

- reducing the ion source output emittance

- optimizing the source collar aperture and dumping the magnetic field profile

These planned development areas are a continuation of the ongoing efforts, which have resulted in the increased ion source performance shown in Figure 9.1. The additional $\sim 10 \%$ source performance improvement will provide operational margin for reliable high-power capability. The present ion source has also demonstrated high reliability over this period $(\sim 99.5 \%)$ and is not a major driver of overall accelerator availability. The path proposed here of incremental improvement of proven technology is deemed lower in risk than the development of a new source technology. For example, earlier SNS power upgrade plans [1] employed a dual ion source solution [2], one operated as a hot spare with a bend magnet in a new downstream magnetic LEBT to switch between sources. The earlier upgrade design aimed for an ion source output current of $67 \mathrm{~mA}$, which would have required a more serious source improvement. Improved chopping scenarios deployed at SNS over the past few years and incorporated into the PPU design significantly reduce the ion source output current requirement. As a result of the lower source requirement and improved production source capability, the development of a dual source and magnetic LEBT (with fast chopping) technology has been dropped from the PPU scope. The change significantly reduces accelerator development requirements.

\subsubsection{Radio Frequency Quadrupole}

The expected RFQ beam transmission is shown in Figure 9.2 for several input beam emittance levels [3]. For the nominal input emittance of 0.25 pi-mm-mrad, the transmission is $\sim 87 \%$ for an input current of $\sim 54 \mathrm{~mA}$. Above $\sim 70 \mathrm{~mA}$, the transmission efficiency begins to drop quickly, but this current level is well above the PPU requirement.

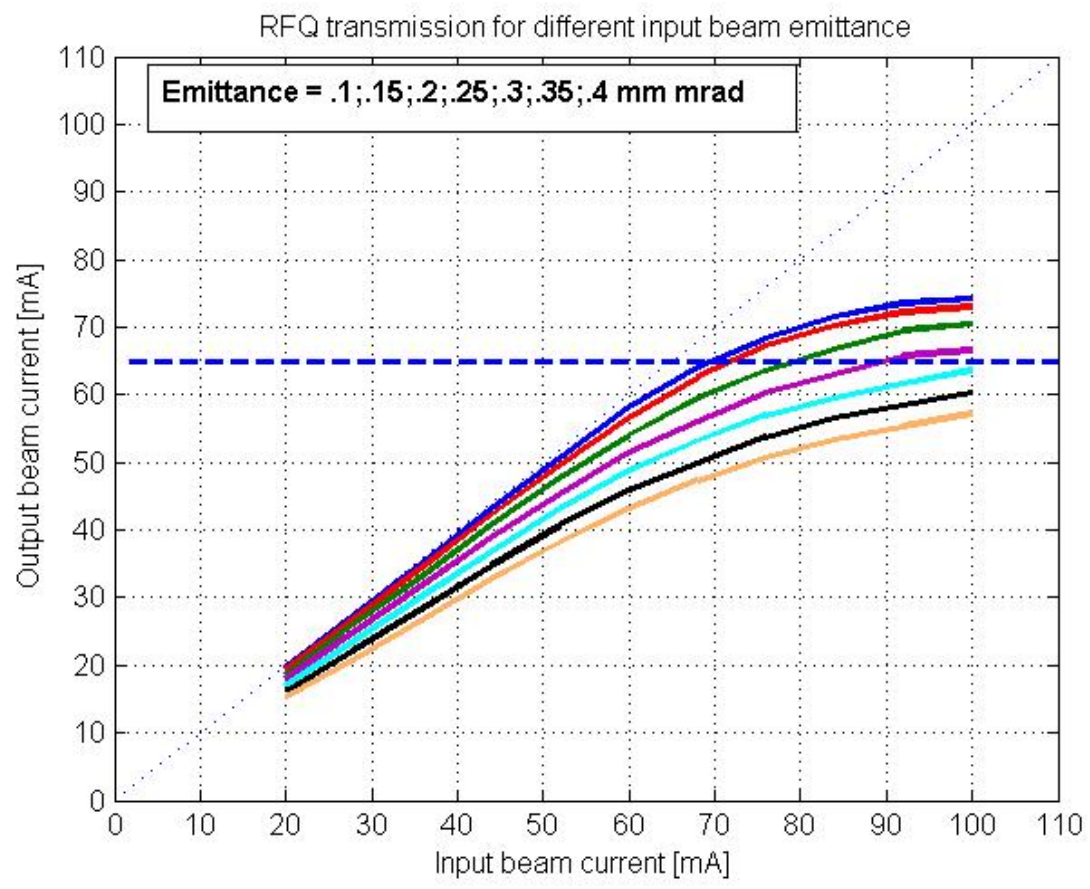

Figure 9.2. RFQ beam transmission from simulations for the design value field profile along the RFQ. 
Nonetheless, $87 \%$ transmission is significantly higher than the measured transmission of $60-80 \%$ in the original RFQ presently installed at SNS. There is a known field profile issue with the present RFQ, which is the original equipment item delivered from Lawrence Berkeley National Laboratory. The present operational RFQ, however, has had several detuning incidents; and the field profile at the RFQ entrance is not known. (After the last detuning incident, the field profile was corrected along most of the RFQ; but measurements were not possible at the RFQ entrance because of access limitations.) To address this problem, a new RFQ was manufactured using the same physics design but incorporating a more robust structure. Therefore, we expect the beam transmission to be the same as for the original RFQ (Figure 9.2). The new RFQ has been delivered and is ready for testing, at high power and with beam, in a test stand. Measurements from this test with beam will provide a basis for the assumptions discussed for the ion source and RFQ performance. If the beam transmission rate is not sufficient for PPU needs, a new RFQ with improved transmission at high currents will be incorporated into the PPU scope.

\subsection{POWER LEVEL}

The PPU project scope is to double the proton beam power capability from 1.4 to $2.8 \mathrm{MW}$. The original accelerator construction provided accommodations for a doubling of the proton power. The path for delivering 2.8 MW uses these accommodations and is described in the STS Technical Design Report [3]. The high-level beam parameters are shown in Table 9.1. Power for a pulsed beam is the product of the beam energy, the macro-pulse beam current, and the duty factor. The PPU choices for the energy and current increase to reach the $2.8 \mathrm{MW}$ power capability have several advantages:

- Ion source beam current is demonstrated.

- It can reach $1.3 \mathrm{GeV}$ with only seven new cryomodules using existing klystrons and coupler designs.

- Only three new modulators are required.

- Most of the warm linac klystrons are capable of accelerating the additional beam current.

- There are modest HVCM improvements from the existing technology (4-5\% increase in capacitor bank voltage).

These choices heavily leverage the accelerator development work done to reach $1.4 \mathrm{MW}$, with minimal further development needed. Although it is possible to design for a higher power than $2.8 \mathrm{MW}$, the project cost and, more critically, project risk increase beyond this level. The factors leading to the choices in Table 9.1 are described in the beam power report [4] and summarized here.

Increasing the beam energy is the most straightforward path to higher beam power. There are advantages to pursuing this path to the extent possible. A higher energy per proton means fewer accelerated protons are required and reduces the probability of beam loss, both from individual particle loss mechanisms and from collective loss mechanisms. At higher beam energy, the collective effects are reduced, so beam loss and instability issues in the storage ring are reduced. However, for beam energies above $1.3 \mathrm{GeV}$, magnetic stripping of the accelerated $\mathrm{H}^{-}$beam in the transport line to the ring begins to exponentially increase to intolerable levels, so the $1.3 \mathrm{GeV}$ beam energy is a rather hard upper limit.

It is possible to increase the beam current beyond the baseline value of $2.8 \mathrm{MW}$; an example is shown in Table 9.1. However, doing so requires development of the ion source capability and the design of a new RFQ to transmit higher beam current. These are primary reasons for limiting the average linac beam current to $38 \mathrm{~mA}$. Also, there are additional burdens on RF equipment (klystrons, modulators, and couplers) due to the additional beam loading, which add to the project cost and technical risks. A major 
complication of this approach is the need to rework many of the presently installed cryomodules with higher-power couplers. There are also increased risks in the foil charge exchange area and beam stability associated with the higher stored beam intensity in the accumulator ring.

Table 9.1. Beam parameters for Proton Power Upgrade options

\begin{tabular}{lccc}
\hline & $\begin{array}{c}\text { 2.8 MW PPU } \\
\text { baseline }\end{array}$ & $\begin{array}{c}\text { 3.5 MW higher } \\
\text { duty factor }\end{array}$ & $\begin{array}{c}\text { 3.5 MW higher } \\
\text { current }\end{array}$ \\
\hline Beam energy & 1.3 & 1.3 & 1.3 \\
Average linac current (mA) & 38 & 38 & 47.5 \\
Peak ion source current (mA) & 54 & 54 & 75 \\
Macro-pulse length (ms) & 1 & 1.25 & 1 \\
Beam repetition rate $(\mathrm{Hz})$ & 60 & 60 & 60 \\
Stored protons in ring $\left(10^{14}\right)$ & 2.26 & 2.83 & 2.83 \\
Stored energy per pulse $(\mathrm{kJ})$ & 47 & 59 & 59 \\
$\begin{array}{l}\text { Normalized ring transverse } \\
\text { space charge }\end{array}$ & 0.78 & 0.98 & 0.98 \\
$\begin{array}{l}\text { Additional high-beta } \\
\text { cryomodules }\end{array}$ & 7 & 7 & $8-9$ \\
$\begin{array}{l}\text { Additional high-voltage } \\
\text { convertor modulators }\end{array}$ & 3 & 3 & 4 \\
\hline
\end{tabular}

It is also possible to increase the linac beam pulse length (duty factor), as indicated in Table 9.1. This approach would significantly increase the technical risk for the ion source reliability and performance capability (it becomes increasingly difficult to produce high currents at increasing duty factors). Also, there are increased risks for the other pulsed RF equipment, such as klystrons and modulators, at higher operational duty factors. The equipment has never been tested beyond the design value corresponding to a $6 \%$ beam duty factor. Also the warm copper RF structures (DTL and CCL) cooling systems were designed for a $6 \%$ duty factor. These systems would have to be reworked to accommodate an increase in the duty factor. Finally, the electrical cost for operation of the RF equipment (a major driver in accelerator electricity usage) is proportional to the duty factor, so a $25 \%$ increase in the duty factor would result in about a $15-20 \%$ increase in utility costs.

Finally, any discussion of increased accelerator power capability should also include a mention of target limitations. As discussed in Chapter 6, the FTS target is being designed for a $2.0 \mathrm{MW}$ capability at $60 \mathrm{~Hz}$. Increasing the accelerator power beyond the $2.8 \mathrm{MW}$ level will potentially benefit the pulses directed toward the STS, but the remaining pulses (40-50 pulses per second from the $60 \mathrm{~Hz}$ stream) directed toward the FTS would be limited in intensity to $33 \mathrm{~kJ} /$ pulse. Thus, a large fraction of the increased beam potential would be underutilized for the pulses directed to the FTS. While it may be possible to develop higher-power FTS targets, and upgrade the FTS support systems to handle higher power, doing so is a high-risk option.

\subsection{REFERENCES}

[1] S. Henderson et al., "Status of the SNS Beam Power Upgrade Project," pp. 345-347 in Proceedings of EPAC 2006, Edinburgh, Scotland, June 26-30, 2006. http://accelconf.web.cern.ch/AccelConf/e06/PAPERS/MOPCH129.PDF. 
[2] B. Han and M. Stockli, "The new LEBT for the SS Power Upgrade Project," pp. 1823-1825 in Proceedings of EPAC 2006, Edinburgh, Scotland, June 26-30, 2006.

http://accelconf.web.cern.ch/AccelConf/p07/PAPERS/TUPAS075.PDF .

[3] J. Galambos, D. E. Anderson, D. Bechtol, et al., Technical Design Report, Second Target Station, ORNL/TM-2015/24, Oak Ridge National Laboratory, January 2015. Available at http://www.osti.gov/scitech/biblio/1185891. Accessed September 2016.

[4] PPU Beam Power Level, PPU-P01-TR0001, internal project technical report, Oak Ridge National Laboratory, November 2016. 



\section{COST AND SCHEDULE}

\subsection{WBS STRUCTURE}

To support the CDR, a WBS was developed to create a logical breakdown of the work by major component and system. All required scope for the completion of the PPU project is included in the project WBS, shown at Level 3 in Figure 10.1.

The WBS is supported by a detailed WBS Dictionary, which defines the scope of work and major deliverables for each node. The WBS and Dictionary are under configuration control, and changes are made according to the PPU Configuration Management Plan.

\subsection{SCHEDULE}

A preliminary project schedule has been developed by decomposing each node of the WBS into discrete activities representing all project work, including research and development, design, procurement, fabrication, construction, installation, and testing. The schedule also includes level-of-effort support activities and milestones and is completely logic driven and resource loaded.

The schedule has incorporated the SNS outage schedule into the plan. Schedule activities are in the process of being optimized to best fit the various outages.

The project duration includes 2 years of activities prior to CD-1 and includes 18 months of schedule float. The actual and planned PPU CD milestone dates are shown in Table 10.1.

Table 10.1. Proton Power Upgrade Critical Decision actual and planned milestones

\begin{tabular}{|c|c|}
\hline Major Milestones & Preliminary Schedule \\
\hline CD-0 (Approved Mission Need) & Jan 2009 (Actual) \\
\hline CD-1/3a (Approved Alternative Selection and Cost Range, and Long Lead Procurements) & 4th Qtr, Year 0 \\
\hline CD-2/3b (Approved Performance Baseline and Start of Construction) & $2^{\text {nd }}$ Qtr, Year 2 \\
\hline CD-4 (Approved Project Completion/Start of Operations) & 4th Qtr, Year 8 \\
\hline
\end{tabular}

At CD-2, the project will establish a Performance Measurement Baseline (PMB), and the baseline schedule will be under configuration control.

\subsection{COST}

The initial cost range of the PPU project is $\$ 184$ million to \$281 million, with a current point estimated total project cost of $\$ 216$ million. The cost estimate includes an $\sim 35 \%$ contingency. The cost ranges are shown in Table 10.2. 


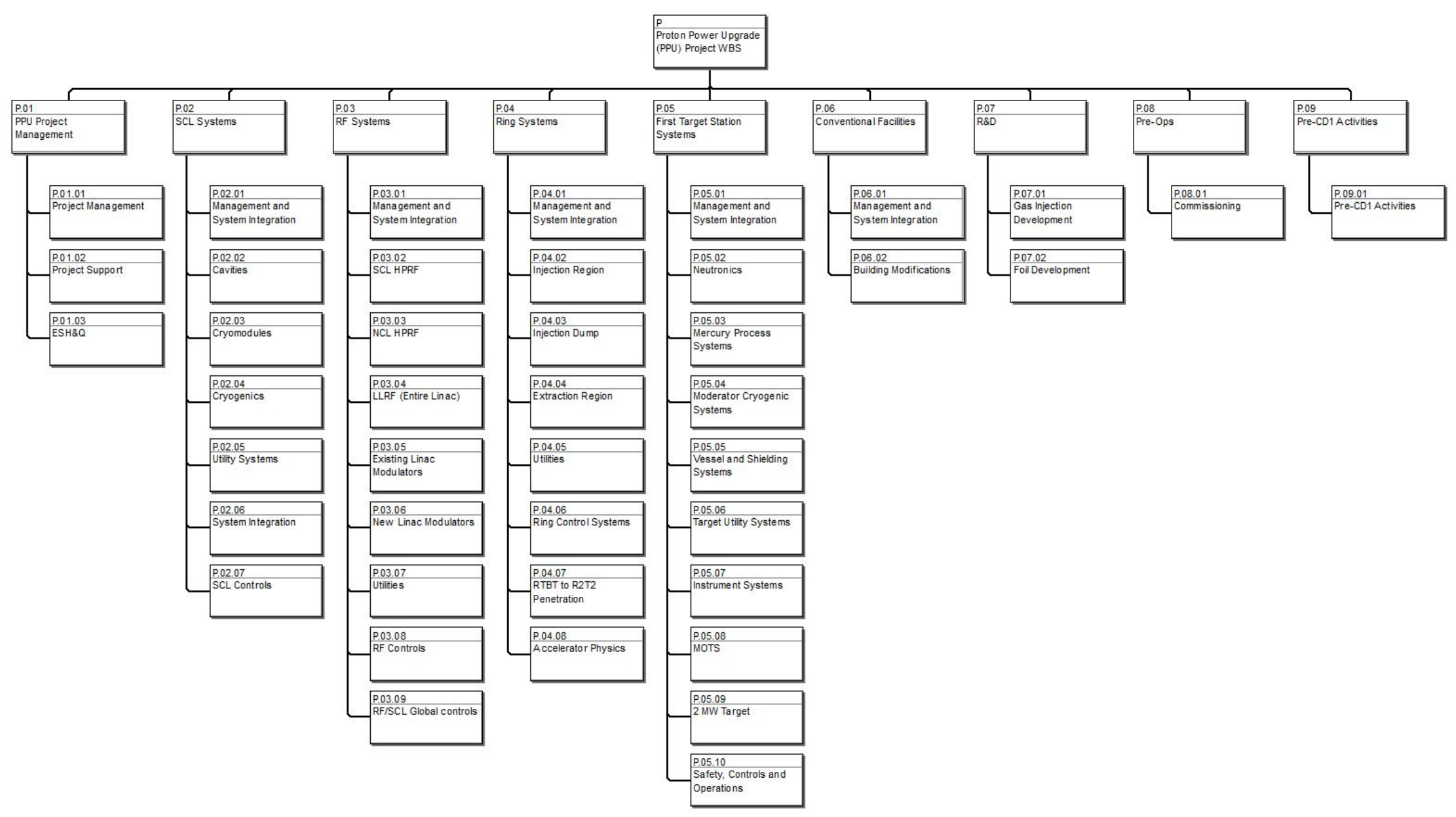

Figure 10.1. Level 3 Work Breakdown Structure for the Proton Power Upgrade. 
Table 10.2. Proton Power Upgrade Project cost estimate ranges

\begin{tabular}{llccc}
\hline WBS & \multicolumn{1}{c}{ Description } & Low (\$M)* & Point (\$M)* & Upper (\$M)* \\
\hline P.01 & Project Management & $\$ 13,273$ & $\$ 15.615$ & $\$ 20,300$ \\
P.02 & SCL Systems & $\$ 40,669$ & $\$ 47,881$ & $\$ 62,245$ \\
P.03 & RF Systems & $\$ 68,920$ & $\$ 81,082$ & $\$ 105,406$ \\
P.04 & Ring Systems & $\$ 11,281$ & $\$ 13,272$ & $\$ 17,254$ \\
P.05 & FTS Systems & $\$ 20,762$ & $\$ 24,426$ & $\$ 31,753$ \\
P.06 & Conventional Facilities & $\$ 9,380$ & $\$ 11,036$ & $\$ 14,346$ \\
P.07 & R\&D & $\$ 3,116$ & $\$ 3,666$ & $\$ 4,766$ \\
P.08 & PreOps & $\$ 811$ & $\$ 954$ & $\$ 1,240$ \\
P.09 & Pre CD-1 Activities & $\$ 2,931$ & $\$ 3,448$ & $\$ 4,482$ \\
\cline { 2 - 5 } & Project Level Risks & $\$ 12,634$ & $\$ 14,864$ & $\$ 19,323$ \\
\cline { 2 - 5 } & Total PPU & $\mathbf{\$ 1 8 3 , 7 7 7}$ & $\mathbf{\$ 2 1 6 , 2 4 4}$ & $\mathbf{\$ 2 8 1 , 1 1 5}$ \\
\hline
\end{tabular}

* Includes escalation and contingency.

The preliminary Budget Authority profile generated from the logic-driven, resource-loaded schedule is shown in Table 10.3.

Table 10.3. Preliminary Budget Authority profile

\begin{tabular}{ccccccccccc}
\hline & Year-1 & Year 0 & Year 1 & Year 2 & Year 3 & Year 4 & Year 5 & Year 6 & Year 7 & Total \\
\hline $\begin{array}{c}\text { Budget Authority } \\
\text { profile (\$M) }\end{array}$ & 1,709 & 6,976 & 27,567 & 70,446 & 33,129 & 40,118 & 26,125 & 7,363 & 2,867 & 216.3 \\
\hline
\end{tabular}

The detailed, bottom-up cost estimate was developed based upon the schedule activities and provides a Bill of Material for each estimated activity. A Basis of Estimate document is also available that captures all systematic estimate assumptions, such as escalation, labor rates, and various instructions regarding estimate technique.

The quantities and durations are largely based upon the actual experience of the engineers, scientists, and technicians who currently operate and maintain the SNS accelerator, target, and CF. Additionally, the majority of the estimated procurements are based upon recent purchases, vendor quotes, and vendor budgetary estimates.

The total project cost includes contingency derived from both estimate uncertainty, which is identified within the cost estimate bills of materials, and event risks, which are captured and quantified in the project risk register.

At CD-2, the project will establish a PMB and the cost estimate will be under configuration control. 
